\title{
Soft Tissue and Bone Sarcoma
}

\author{
Edited by \\ Edwin Choy \\ Printed Edition of the Special Issue Published in Cancers
}

MDPI www.mdpi.com/journal/cancers 
Edwin Choy (Ed.)

\section{Soft Tissue and Bone Sarcoma}

\section{MDPI}


This book is a reprint of the special issue that appeared in the online open access journal Cancers (ISSN 2072-6694) in 2012 (available at:

http://www.mdpi.com/journal/cancers/special_issues/bone-sarcoma).

Guest Editor

Edwin Choy

Division of Hematology and Oncology

Massachusetts General Hospital

P.O. Box 226

Boston, MA 02114, USA

\section{Editorial Office}

MDPI AG

Klybeckstrasse 64

4057 Basel, Switzerland

Publisher

Shu-Kun Lin

Production Editor

Martyn Rittman

\section{Edition 2014}

MDPI • Basel • Beijing • Wuhan

ISBN 978-3-906980-58-4 (PDF)

ISBN 978-3-906980-46-1 (Hbk)

(C) 2014 by the authors; licensee MDPI AG, Basel, Switzerland. All articles in this volume are Open Access distributed under the Creative Commons License (CC BY), which allows users to download, copy and build upon published articles, even for commercial purposes, as long as the author and publisher are properly credited. The dissemination and distribution of physical copies of this book as a whole, however, is restricted to MDPI AG, Basel, Switzerland. 


\section{Table of Contents}

Preface V

\section{Section One: Systemic Therapies for Sarcoma}

William W. Tseng, Neeta Somaiah, Alexander J. Lazar, Dina C. Lev and Raphael E. Pollock

Novel Systemic Therapies in Advanced Liposarcoma: A Review of Recent Clinical Trial Results

Reprinted from: Cancers 2013, 5(2), 529-549

http://www.mdpi.com/2072-6694/5/2/529 1

Claire F. Verschraegen, Sujana Movva, Yongli Ji, Berndt Schmit, Robert H. Quinn, Ben Liem, Therese Bocklage and Monte Shaheen

A Phase I Study of the Combination of Temsirolimus with Irinotecan for Metastatic Sarcoma Reprinted from: Cancers 2013, 5(2), 418-429 http://www.mdpi.com/2072-6694/5/2/418

\section{Section Two: Multidisciplinary Treatment of Sarcomas}

\section{Matthew M. Ladra and Torunn I. Yock}

Proton Radiotherapy for Pediatric Sarcoma

Reprinted from: Cancers 2014, 6(1), 112-127

http://www.mdpi.com/2072-6694/6/1/112

Mohamedtaki A. Tejani, Thomas J. Galloway, Miriam Lango, John A. Ridge and Margaret von Mehren

Head and Neck Sarcomas: A Comprehensive Cancer Center Experience

Reprinted from: Cancers 2013, 5(3), 890-900

http://www.mdpi.com/2072-6694/5/3/890 


\section{Section Three: Sarcoma Biology}

Teresa Infante, Elena Cesario, Michele Gallo, Flavio Fazioli, Annarosaria De Chiara, Cristina Tutucci, Gaetano Apice and Filomena de Nigris

Ex Vivo Behaviour of Human Bone Tumor Endothelial Cells

Reprinted from: Cancers 2013, 5(2), 404-417

http://www.mdpi.com/2072-6694/5/2/404

Atta Goudarzi, Nalan Gokgoz, Mona Gill, Dushanthi Pinnaduwage, Daniele Merico, Jay S. Wunder and Irene L. Andrulis

Protein Kinase C Epsilon and Genetic Networks in Osteosarcoma Metastasis

Reprinted from: Cancers 2013, 5(2), 372-403

http://www.mdpi.com/2072-6694/5/2/372

\section{Navid Sadri and Paul J. Zhang}

Hypoxia-Inducible Factors: Mediators of Cancer Progression; Prognostic and Therapeutic Targets in Soft Tissue Sarcomas

Reprinted from: Cancers 2013, 5(2), 320-333

http://www.mdpi.com/2072-6694/5/2/320

Nicholas M. Bernthal, Kevin B. Jones, Michael J. Monument, Ting Liu, David Viskochil and R. Lor Randall

Lost in Translation: Ambiguity in Nerve Sheath Tumor Nomenclature and Its Resultant

Treatment Effect

Reprinted from: Cancers 2013, 5(2), 519-528

http://www.mdpi.com/2072-6694/5/2/519

Kim M. Boerkamp, Gerard R. Rutteman, Marja J. L. Kik, Jolle Kirpensteijn, Christoph Schulze and Guy C. M. Grinwis

Nuclear DNA-Content in Mesenchymal Lesions in Dogs: Its Value as Marker of Malignancy and Extent of Genomic Instability

Reprinted from: Cancers 2012, 4(4), 1300-1317

http://www.mdpi.com/2072-6694/4/4/1300 


\section{Preface}

Soft tissue and bone sarcomas disproportionately affect children, adolescents, and young adults. Although considered a rare disease, sarcomas continue to have a devastating effect on these patients and their loved ones, and their impact on our society far exceed their relatively low prevalence. Because of its rarity and heterogeneity, clinical decision making on management of sarcomas are often individualized and without a consensus treatment strategy. Furthermore, research to improve clinical outcomes are particularly challenging as sizeable studies are hard to produce. By improving our knowledge about the molecular biology of sarcomas, we hope to identify new treatment strategies and targets for further drug development.

In this Special Issue in Soft Tissue and Bone Sarcomas, we invited manuscripts that sought to elucidate the pathophysiology and carcinogenesis of soft tissue and bone sarcomas and identify new arenas for sarcoma research. We were particularly interested in manuscripts deciphering the mechanisms of putative targets for sarcoma treatments. By devoting a special issue solely on soft tissue and bone sarcomas, we hoped to display and bridge a critical mass of new and exciting research that can lead the way to improving cancer care for this cohort of patients.

The breadth and quality of manuscripts submitted for our review far exceeded our expectations. We were able to include review articles as well as primary research manuscripts. Our papers encompass studies of clinical, translational, and basic science. We included studies describing investigations of sarcomas in vitro as well as in vivo, in dogs as well as in humans, systemic treatments as well as radiation therapies, and a det ailed description of a clinical trial as well as an overview of multiple clinical trials. The richness of articles that we were able to publish in this collection underlies the growing enthusiasm to study, collaborate, and take scientific risks in studying sarcomas. We were often told that our field is too rare, too heterogeneous, or too far from the mainstream cancer conversation that our research is not worth funding, publishing, or publicizing, but times are changing. I feel that, as a community of scientists, sarcoma investigators have finally reached the critical mass required in all disciplines to raise the level of research to that elusive next level.

Edwin Choy, MD, PhD

Guest Editor 

Reprinted from Cancers. Cite as: Tseng, W.W.; Somaiah, N.; Lazar, A.J.; Lev, D.C.; Pollock, R.E. Novel Systemic Therapies in Advanced Liposarcoma: A Review of Recent Clinical Trial Results. Cancers 2013, 5, 529-549.

Review

\title{
Novel Systemic Therapies in Advanced Liposarcoma: A Review of Recent Clinical Trial Results
}

\author{
William W. Tseng ${ }^{1}$, Neeta Somaiah ${ }^{2}$, Alexander J. Lazar ${ }^{3}$, Dina C. Lev ${ }^{4}$ and \\ Raphael E. Pollock ${ }^{1,4, *}$
}

1 Department of Surgical Oncology, The University of Texas M.D. Anderson Cancer Center, 1515 Holcombe Blvd, Houston, TX 77030, USA; E-Mail: wtseng@mdanderson.org

2 Department of Sarcoma Medical Oncology, The University of Texas M.D. Anderson Cancer Center, 1515 Holcombe Blvd, Houston, TX 77030, USA; E-Mail: nsomaiah@mdanderson.org

3 Department of Pathology, The University of Texas M.D. Anderson Cancer Center, 1515 Holcombe Blvd, Houston, TX 77030, USA; E-Mail: alazar@mdanderson.org

4 Department of Cancer Biology, The University of Texas M.D. Anderson Cancer Center, 1515 Holcombe Blvd, Houston, TX 77030, USA; E-Mail: dlev@mdanderson.org

* Author to whom correspondence should be addressed; E-Mail: rpollock@mdanderson.org; Tel.: +1-713-792-6928; Fax: +1-713-563-4637.

Received: 21 March 2013; in revised form: 16 April 2013 / Accepted: 2 May 2013 / Published: 10 May 2013

\begin{abstract}
Liposarcoma is one of the most common adult soft tissue sarcomas and consists of three histologic subtypes (well and dedifferentiated, myxoid/round cell, and pleomorphic). Surgery is the mainstay of treatment for localized disease; however for unresectable or metastatic disease, effective treatment options are currently limited. In the past decade, a better understanding of the distinct genetic and molecular aberrations for each of the three histologic subtypes has led to the development of several novel systemic therapies. Data from phase I and early phase II clinical trials have been reported. Despite challenges with conducting clinical trials in liposarcoma, preliminary results for several of these novel, biology-driven therapies are encouraging.
\end{abstract}

Keywords: liposarcoma; chemotherapy; molecular-based therapy; clinical trials 


\section{Introduction}

Soft tissue sarcomas are a heterogeneous group of over 50 different malignancies of mesenchymal origin [1]. In adults, liposarcoma is one of the most common types of soft tissue sarcoma [2]. Within liposarcoma, three distinct histologic subtypes are recognized by the World Health Organization: (1) well and dedifferentiated, (2) myxoid/round cell, and (3) pleomorphic liposarcoma [2]. Although surgical resection is the mainstay of treatment for localized disease in all subtypes, many patients with liposarcoma will initially present with or ultimately progress to advanced disease that is either unresectable, metastatic or both. For these patients, the mortality is high and local and/or systemic tumor burden may also cause significant morbidity. As a method of local control, radiation therapy can provide symptom palliation for a small subset of patients, but systemic disease is not addressed $[3,4]$. Current cytotoxic chemotherapy can potentially provide systemic control, but toxicity tends to be quite high $[3,4]$. As a result, for the majority of liposarcoma patients with advanced disease, treatment options are currently limited.

In this review, we will provide an overview of liposarcoma followed by a brief discussion of conventional cytotoxic chemotherapy and commonly adopted endpoints for treatment efficacy. We will then summarize the results of recent clinical trials with novel systemic therapies for patients with advanced liposarcoma. Many of published results are from studies done in the context of the broader group of adult soft tissue sarcoma; however we will focus on liposarcoma. As we will discuss, even within liposarcoma, recognition of the specific histologic subtype is critical, especially as novel therapies are emerging based on a better understanding of subtype-specific disease biology.

\section{Three Distinct Liposarcoma Subtypes}

In contrast to benign lipomas, all three subtypes of liposarcoma are true adipocytic malignancies which can cause significant morbidity and mortality. Each subtype is characterized by distinct genetic and molecular aberrations and unique histologic appearance, suggesting separate pathways to malignant transformation [2-4]. Accordingly, the initial presentation, pattern of disease progression and overall clinical outcome varies with each liposarcoma subtype. An understanding of these multi-level differences (Table 1) is critical to the management of the liposarcoma patient and selection of appropriate treatment options.

Well-differentiated (WD) and dedifferentiated (DD) liposarcoma are the most common subtype of liposarcoma [2]. Both WD and DD tumor cells exhibit amplification of chromosome 12q13-15, a region which contains several hundred genes including MDM2, an inhibitor of the tumor suppressor p53, and CDK4, a critical regulator of cell cycling. By histology, WD liposarcoma are characterized by the presence of adipocytes of varying sizes with prominent fibrous stroma (Figure 1A); lipoma-like, sclerosing, and inflammatory variants have also been described [2]. DD liposarcomas typically have an adipocyte-rich, WD portion that is well demarcated from a highly cellular, spindle cell-rich DD portion (Figure 1B). To establish a definitive histologic diagnosis of DD, five or more mitoses per 10 high power fields are required [2]. The majority of DD cases are found de novo, but up to $25 \%-40 \%$ of patients with WD will ultimately manifest DD histology at recurrence [5]. DD liposarcoma was traditionally thought to arise from WD; however the exact clonal relationship 
between WD and DD liposarcoma is not clear. Presence of DD histology nonetheless is associated with much more aggressive disease and worse clinical outcome $[5,6]$.

Table 1. Important multi-level differences in liposarcoma subtypes.

\begin{tabular}{|c|c|c|c|c|c|}
\hline $\begin{array}{l}\text { Liposarcoma } \\
\text { Histologic } \\
\text { Subtype } \\
\end{array}$ & $\begin{array}{l}\text { Genetic and/or } \\
\text { Molecular } \\
\text { Aberration } \\
\end{array}$ & Histologic Features & Anatomic Site & $\begin{array}{l}\text { Clinical } \\
\text { Behavior }\end{array}$ & $\begin{array}{l}\text { Response } \\
\text { to Current } \\
\text { Therapy }\end{array}$ \\
\hline $\begin{array}{l}\text { Well } \\
\text { differentiated } \\
\text { (WD) }\end{array}$ & $\begin{array}{l}\text { 12q13-15 } \\
\text { amplification (MDM2, } \\
\text { CDK4, etc.) }\end{array}$ & $\begin{array}{l}\text { Adipocytes of varying } \\
\text { size, prominent fibrous } \\
\text { stroma }\end{array}$ & $\begin{array}{l}\text { Retroperitoneum } \\
\text { >extremities, } \\
\text { paratesticular areas, } \\
\text { trunk }\end{array}$ & $\begin{array}{l}\text { Locoregional } \\
\text { recurrence }\end{array}$ & Poor \\
\hline $\begin{array}{l}\text { Dedifferentiated } \\
\text { (DD) }\end{array}$ & same as WD & $\begin{array}{l}\text { Highly-cellular portion } \\
\text { ( } 5 \text { or more mitoses/10 } \\
\text { HPF) next to WD } \\
\text { portion }\end{array}$ & same as WD & $\begin{array}{l}\text { Locoregional } \\
\text { recurrence and } \\
\text { distant metastasis } \\
(10 \%-15 \%)\end{array}$ & Low \\
\hline $\begin{array}{l}\text { Myxoid/Round } \\
\text { Cell (MRC) }\end{array}$ & $\begin{array}{l}\text { Translocation } \\
(\mathrm{t} 12 ; 16)(\mathrm{q} 13 ; \mathrm{p} 11) \text { or } \\
(\mathrm{t} 12 ; 22)(\mathrm{q} 13 ; \mathrm{q} 12) \\
\text { leading to } \\
\text { FUS-CHOP/DDIT3 or } \\
\text { EWS-CHOP/DDITS } \\
\text { fusion protein }\end{array}$ & $\begin{array}{l}\text { Abundant extracellular } \\
\text { myxoid material; } \\
\text { sparse cellular portion } \\
\text { w/mature adipocytes, } \\
\text { immature lipoblasts, } \\
\text { round cells ( }>5 \% \text { of } \\
\text { tumor) }\end{array}$ & $\begin{array}{l}\text { Proximal lower } \\
\text { extremities }\end{array}$ & $\begin{array}{l}\text { Distant metastasis } \\
(10 \%-20 \%) \text { to } \\
\text { visceral organ } \\
\text { sites, bone, and } \\
\text { fat bearing areas }\end{array}$ & High \\
\hline Pleomorphic & Complex & $\begin{array}{l}\text { Highly cellular } \\
\text { resembling MFH; } \\
\text { pleomorphic lipoblasts; } \\
\text { occassional } \\
\text { multinucleated cells }\end{array}$ & $\begin{array}{l}\text { Lower extremities } \\
\text { >retroperitoneum; } \\
\text { mediastinum }\end{array}$ & $\begin{array}{l}\text { Distant metastasis } \\
(30 \%-50 \%)\end{array}$ & Low \\
\hline
\end{tabular}

WD and DD liposarcoma does not have a certain age or gender predilection and there are no known risk factors (e.g., obesity) for the development of disease. WD and DD liposarcoma most commonly occur in the retroperitoneum and to a lesser extent, the extremities, paratesticular areas, and trunk. WD liposarcoma does not metastasize, whereas DD liposarcoma has the potential for distant metastasis, typically to the lungs. The true frequency of this event, however, has been estimated to b e 10\%-15\% [7]. For both WD and DD, the majority of patients experience locoregional morbidity. In the retroperitoneum, tumors can be massive in size $(>30 \mathrm{~cm})$ and/or invade adjacent viscera and structures, making surgical resection challenging. Locoregional recurrence is very common and patients often undergo multiple re-operations. WD and DD liposarcoma are largely resistant to conventional cytotoxic chemotherapy and radiation therapy [2-4], and as a result, treatment options other than surgery, are limited.

Myxoid/round cell (MRC) liposarcoma is the second most common subtype of liposarcoma. In terms of genetic abnormalities, MRC liposarcoma is characterized by translocation of chromosomes 12 and $16(\mathrm{t} 12 ; 16)(\mathrm{q} 13 ; \mathrm{p} 11)$, that results in a fusion gene arrangement between FUS and CHOP/DDIT3. In rare cases, an alternative translocation event can occur $(\mathrm{t} 12 ; 22)(\mathrm{q} 13 ; \mathrm{q} 12)$, that results in an EWS-CHOP fusion gene. FUS- and EWS-CHOP/DDIT3 are both thought to interfere 
with normal adipocytic differentiation through the C/EBP family of transcription factors and are likely involved in activation of a number of tyrosine kinase receptor pathways including MET, RET and PI3K/Akt. By histology, MRC liposarcoma is notable for abundant extracellular myxoid material with typically, a sparse cellular component consisting of mature adipocytes, immature lipoblasts and round cells (Figure 1C) [2]. In a subset of cases, the cellularity increases with a predominance of round cells containing a high nuclear to cytoplasmic ratio (Figure 1D). Round cell transformation - defined as $>5 \%$ of the tumor-is associated with more aggressive disease biology and worse clinical outcome [8].

Figure 1. Representative photomicrographs of well differentiated (A), dedifferentiated (B), myxoid (C)/round cell (D), and pleomorphic (E) liposarcoma. A challenging case is shown in (F). This portion of a well differentiated liposarcoma shows myxoid features, however other areas of the tumor showed characteristic features and 12q13-15 amplification.
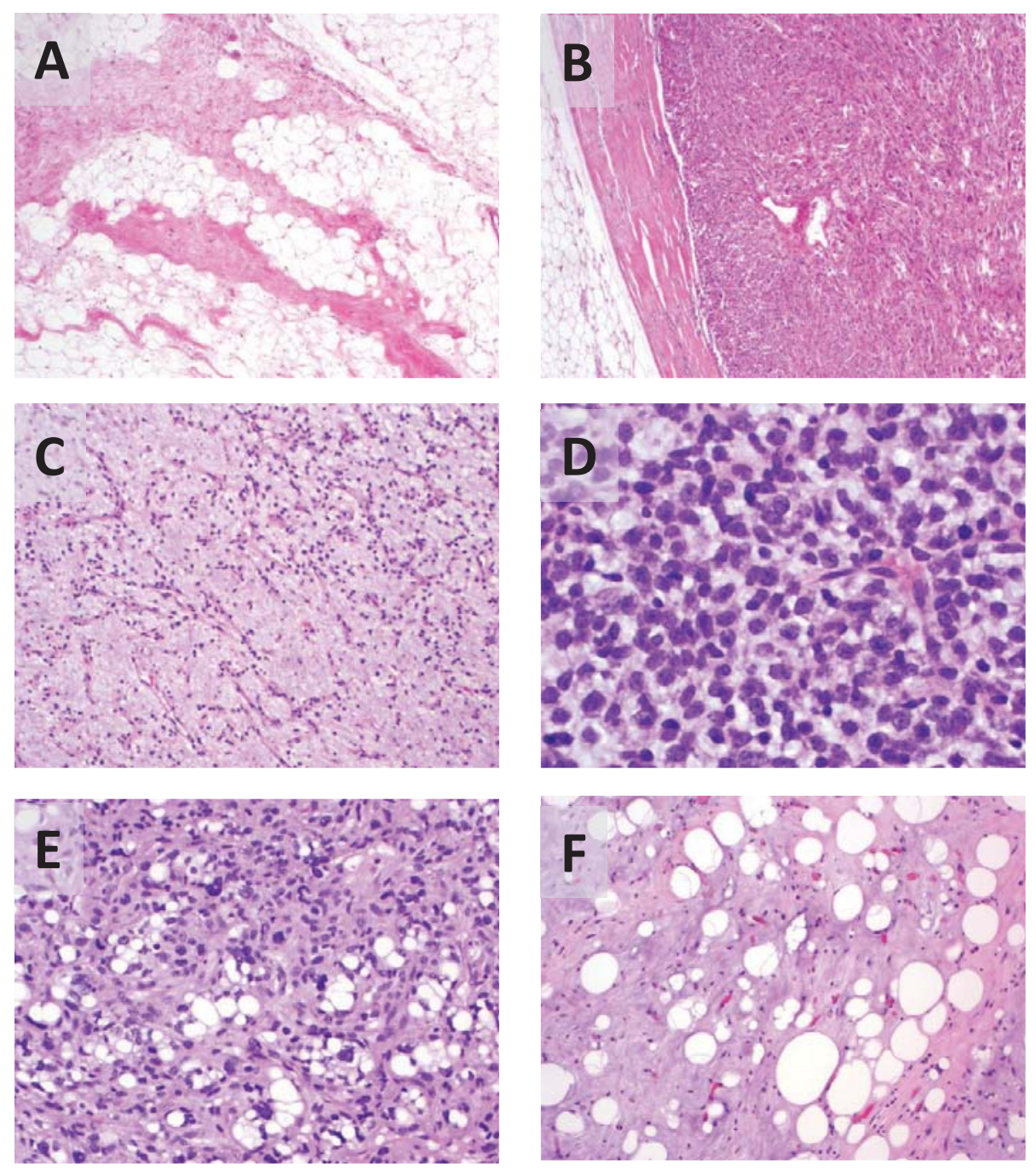

MRC liposarcoma tends to present in younger patients and affects the proximal lower extremities as opposed to the retroperitoneum. In fact, intraabdominal and retroperitoneal disease may actually be WD/DD liposarcoma that was misdiagnosed [9]. Distant dissemination to visceral organ sites 
(e.g., lung) occurs in 10\%-20\% of patients, especially with round cell transformation. Patients should be carefully monitored for skeletal metastases (e.g., w/MRI), which may represent up to half of all metastatic events [10]. Unique among the liposarcoma subtypes, MRC liposarcoma also appears to have a predilection for metastases to fat-bearing areas in the retroperitoneum, chest, trunk and other extremities $[11,12]$. Several studies have established that tumors at distant fat-bearing sites are in fact, metastases from the same clonal origin as the primary site, as opposed to multifocal sites of disease [13,14]. Treatment for MRC liposarcoma consists of surgical resection for localized, primary disease. In patients with advanced or metastatic disease, MRC liposarcoma is known for its sensitivity to radiation therapy [11] and cytotoxic chemotherapy [15] in comparison to the other liposarcoma subtypes [16].

Pleomorphic liposarcoma is the third, least common and least understood subtype of liposarcoma. A single characteristic genetic abnormality has not yet been identified; instead, complex changes are seen with chromosomal duplications, gains, losses, and rearrangements. By histology, pleomorphic liposarcoma resembles a non-adipocytic soft tissue sarcoma called malignant fibrous histiocytoma (MFH), also known as undifferentiated pleomorphic sarcoma (UPS), with high cellularity and additionally, presence of pleomorphic lipoblasts and occasional multinucleated giant cells (Figure 1E) [2]. An epithelioid histologic variant resembling renal cell or adrenocortical carcinoma has also been described [17]. Patients with pleomorphic liposarcoma most commonly present with disease in the lower extremity and occasionally at other sites, including the retroperitoneum and mediastinum. Disease progression is much more aggressive compared to the other liposarcoma subtypes, with a higher $(30 \%-50 \%)$ frequency of distant metastasis to visceral organ sites, including lung, bone and liver. Tumors are highly resistant to all current treatment modalities $[18,19]$.

Despite hallmark genetic aberrations and characteristic histologic features, in practice, distinguishing one liposarcoma subtype from another can at times be challenging (Figure 1F). Histologic examination, ideally by an experienced soft tissue sarcoma pathologist, is best supplemented with molecular studies (e.g., MDM2 amplification by fluorescence in situ hybridization in WD/DD liposarcoma) for accurate diagnosis. Molecular studies can frequently lead to reclassification of incorrectly diagnosed cases [20,21]. An accurate diagnosis is critical not only to counsel patients regarding likely disease course (e.g., frequent locoregional recurrence in retroperitoneal WD/DD liposarcoma; metastases to fat-bearing areas in MRC) but for decision making with regard to available treatment options or enrollment into clinical trials for those with advanced disease.

\section{Conventional Cytotoxic Chemotherapy}

The current cytotoxic chemotherapy agents for unresectable/metastatic liposarcoma are based on efficacy data from trials encompassing all soft tissue sarcoma subtypes. Single agent anthracycline (mainly doxorubicin) or an anthracycline-based combination is considered the standard for first-line therapy in patients with advanced disease [22]. Other agents with single agent activity that are frequently combined with doxorubicin are ifosfamide and dacarbazine. The objective response rate (ORR) in treatment naïve soft tissue sarcoma patients is somewhere between $18 \%$ to $38 \%$ [23-30]. Although anthracycline based combinations tend to have higher response rates compared to single agent therapy, a survival benefit has not been demonstrated in the trials so far $[25,26]$. This might be 
due to the studies having inadequate power to detect the survival advantage or due to the higher toxicity and limited added efficacy of the cytotoxic agent used in the combination.

Gemcitabine and docetaxel is a frequently used non-anthracycline combination in the second-line setting for liposarcomas. A randomized phase II study conducted by Maki et al. in metastatic soft tissue sarcoma suggested a survival benefit for fixed-dose rate gemcitabine with docetaxel over fixed-dose rate gemcitabine alone [31]. The median progression-free survival (PFS) and overall survival (OS) of gemcitabine therapy alone was 3 months and 18 months compared to 6 months and 12 months with the combination, respectively. The best responses were seen in leiomyosarcoma and undifferentiated pleomorphic sarcoma (UPS/MFH) patients. Among the small number of liposarcoma patients ( $\mathrm{n}=20,16 \%$ of all patients), only two (both WD/DD) had stable disease at 6 months with gemcitabine alone. An additional five patients (three WD/DD, two MRC) with gemcitabine alone and five patients (four WD/DD, one pleomorphic) with combination therapy had stable disease for less than 6 months.

To date, no prospective trial with conventional cytotoxic chemotherapy agents has individually assessed response in a liposarcoma patient cohort alone. Retrospective, subtype specific studies have been reported which reflect response in liposarcoma patients and also highlight the variability between the three major liposarcoma subtypes. In these studies, the combination of doxorubicin and ifosfamide resulted in good response rates in MRC liposarcoma (ORR $=43 \%$ ) and is hence the treatment of choice for this subtype [32]. Italiano et al. reported a multicenter, retrospective study of $208 \mathrm{~W} \mathrm{D}$ and DD liposarcoma patients, $82 \%$ of which were treated with an anthracycline-containing regimen. The ORR was only $12 \%$ and all of the responses occurred in anthracycline treated patients. Rates of 3- and 6 month PFS were 59\% and 44\% [33]. In pleomorphic liposarcoma, Italiano et al. reported an ORR of $37 \%$ with various cytotoxic chemotherapy regimens with no significant difference between the various single agents or combination regimens used [34]. Of note, only 32 patients over a ten year period were assessable for response, attesting to the rarity of this disease. Rates of 3 - and 6 month PFS were $63 \%$ and $43 \%$.

For all soft tissue sarcomas in general, despite objective response rates, a median survival of 8 to 13 months is estimated from the start of first-line anthracycline-based chemotherapy, as shown in randomized studies performed over the last two decades [24,27,30,35]. The median survival for patients for whom conventional chemotherapy with an anthracycline and ifosfamide has failed is in the range of 6 months [28,36,37]. Most recent studies therefore, focus on the progression free rate (PFR) when evaluating agents for anti-tumor activity. A commonly used reference benchmark for an active agent in soft tissue sarcoma was proposed by Van Glabbeke et al. [38]. Based on analysis of fourteen clinical trials of cytotoxic therapies conducted by the EORTC, the PFR for active and inactive agents for soft tissue sarcomas (all histologies together) in the first and second line setting was determined. For first-line therapy, a 6-month PFR of 30\% or higher suggested drug activity. For second-line therapy, a 3-month PFR of $40 \%$ or higher was associated with an active drug and $20 \%$ or lower meant inactivity. A recent review of published series confirmed that ifosfamide, dacarbazine-gemcitabine and docetaxel-gemcitabine meet these disease stabilization criteria as second-line therapies [22]. 


\section{Novel Systemic Therapies in Liposarcoma}

In the past decade, results from clinical trials have identified several novel systemic therapies in soft tissue sarcoma, many of which have potential efficacy in liposarcoma (Table 2). In contrast to conventional cytotoxic chemotherapies, which are non-specific, the majority of these novel therapies are based on the understanding of disease biology inherent to a given sarcoma histology, in many cases targeting a specific, aberrant genetic or molecular pathway.

Table 2. Overview of reported human studies and clinical trials for novel systemic therapies in liposarcoma.

\begin{tabular}{|c|c|c|c|c|}
\hline $\begin{array}{l}\text { Novel } \\
\text { Therapy }\end{array}$ & $\begin{array}{l}\text { Mechanism of } \\
\text { Action }\end{array}$ & $\begin{array}{l}\text { Liposarcoma } \\
\text { Histologic } \\
\text { Subtype }\end{array}$ & $\begin{array}{l}\text { Study } \\
\text { Type/Clinical } \\
\text { Trial Phase } \\
\end{array}$ & $\begin{array}{l}\text { References }^{\wedge} \\
(\mathbf{n}=\text { liposarcoma pts })\end{array}$ \\
\hline Trabectedin & $\begin{array}{l}\text { Binding of DNA } \\
\text { minor groove; direct } \\
\text { interaction } \\
\text { w/FUS-CHOP }\end{array}$ & MRC & $\begin{array}{l}\text { Phase II, } \\
\text { Retrospective, } \\
\text { and Neoadjuvant }\end{array}$ & $\begin{array}{l}\text { Garcia-Cabonero, } 2004 \text { (10); } \\
\text { Yovine, } 2004 \text { (6); Le Cesne, } 2005 \\
\text { (10); Grosso, } 2007 \text { (51 *); } \\
\text { Grosso, } 2009 \text { (32 *); Demetri, } \\
2009 \text { (93); Gronchi, } 2012(23 *), \\
\text { Samuels, } 2013 \text { (233) }\end{array}$ \\
\hline Eribulin & Microtubule inhibitor & DD & Phase II & Schoffski, 2011 (37) \\
\hline RG7112 & MDM2 antagonist & WD/DD & $\begin{array}{l}\text { Phase I } \\
\text { (Neoadjuvant) }\end{array}$ & Ray-Coquard, 2012 (20*) \\
\hline Flavopiridol & $\begin{array}{l}\text { pan-CDK inhibitor, } \\
\text { including CDK4 }\end{array}$ & WD/DD & Phase I & Luke, 2012 (16) \\
\hline PD 0332991 & CDK4/6 inhibitor & $\mathrm{WD} / \mathrm{DD}$ & Phase I & Schwartz, 2012 (7) \\
\hline $\begin{array}{l}\text { Troglitazone, } \\
\text { Rosiglitazone, } \\
\text { Efatutazone } \\
\end{array}$ & $\begin{array}{l}\text { PPAR-gamma } \\
\text { agonist }\end{array}$ & all & Phase I, II & $\begin{array}{l}\text { Debrock, } 2003(12 *) \text {; Pishvaian, } \\
2012 \text { (5) }\end{array}$ \\
\hline Nelfinavir & SREBP-1 inhibitor & WD/DD & Phase I & Pan, $2012(20 *)$ \\
\hline $\begin{array}{l}\text { Pazopanib, } \\
\text { Sorafenib, } \\
\text { Sunitinib }\end{array}$ & $\begin{array}{l}\text { Tyrosine kinase } \\
\text { receptor inhibitor }\end{array}$ & all & Phase II & $\begin{array}{l}\text { Sleijfer, } 2009 \text { (19); von Mehren, } \\
2012 \text { (10); Tariq Mahmood, } 2011 \\
(17)\end{array}$ \\
\hline
\end{tabular}

$\wedge=$ reports with $\mathrm{n}=5$ or less patients were excluded, but are described in the text. $*=$ indicates the study was focused on liposarcoma only.

\subsection{Marine-Derived Compounds-Trabectedin and Eribulin}

Trabectedin (Yondelis ${ }^{\mathrm{TM}} /$ Ecteinascidin-743) is a tetrahydroisoquinoline alkaloid compound, originally derived from the Carribean sea tunicate, Ecteinascidia turbinata [39]. Among the novel systemic therapies in liposarcoma, trabectedin is the most well-studied in clinical trials and thus far, has the highest treatment efficacy.

The primary mechanism of action for trabectedin is through binding of the DNA minor groove, causing structural changes and irreversible DNA damage that leads to cell cycle arrest and apoptosis. Mechanistic studies have demonstrated that trabectedin may have target specificity in MRC liposarcoma by direct interaction with the FUS-CHOP fusion protein, preventing its binding to 
transcriptional promoters and restoring normal lipoblast maturation [40]. Recent studies also suggest that trabectedin may have anti-inflammatory effects and specifically target tumor-associated macrophages [41,42].

Three prospective phase II studies initially established the therapeutic potential of trabectedin in patients with advanced soft tissue sarcomas. A closer scrutiny of enrolled patients in these studies, however, demonstrates that very few actually had liposarcoma. Garcia-Carbonero et al. reported on 36 patients, only 10 (28\%) of which had liposarcoma (histologic subtypes not specified) [43]. Nonetheless, major objective responses were seen in two of these patients (one MRC, one DD), including complete response in the patient with MRC liposarcoma. Yovine et al. had only six liposarcoma patients (11\% of total) in their study, one of whom had stable disease for 31 months [44]. Le Cesne et al. had 10 liposarcoma patients (10\% of total), four of whom had either stable disease or partial response [45].

Demetri et al. subsequently reported results of a larger phase II study which compared two different dosing schedules of trabectedin (intravenous administration over $3 \mathrm{~h}$ e very week vs. $24 \mathrm{~h}$ every 3 weeks) in patients with leiomyosarcoma or liposarcoma, the latter of which had 93 patients (34\%, subtype not specified) [46]. Trabectedin given for $24 \mathrm{~h}$ every 3 weeks was found to be superior, with 3 month PFR of 52\% and 6 month PFR of $36 \%$ for all study patients; subanalysis of the liposarcoma patients alone was not reported.

Recently, Samuels et al. reported on RECIST objective response rates and overall survival for trabectedin in previously-treated patients with advanced soft tissue sarcoma, including 233 patients with liposarcoma (21\% of total, subtype not specified), representing the largest series to date [47]. ORR for liposarcoma patients in this study was $6 \%$, including seven patients with partial response and one patient with complete response. Patients with liposarcoma had a median overall survival of 18.1 months, compared to 11.9 for all evaluable patients. PFR was not reported.

The treatment efficacy of trabectedin was evaluated specifically for MRC liposarcoma in a multicenter, retrospective study reported by Grosso et al. [48]. Data was analyzed from 51 previously-treated patients with locally-advanced and metastatic disease who were enrolled in a compassionate use program. Remarkably, the PFR at 3 months was $92 \%$ and at 6 months, $88 \%$. In addition, by RECIST criteria, 24 patients experienced partial response and two patients had complete response $(\mathrm{ORR}=51 \%)$. The authors noted that in the majority of responders, changes in tissue density were seen on radiographic imaging prior to tumor shrinkage. Subsequent long-term analysis in the subset of 32 patients from the Milano group confirmed the durability of these results [49].

Use of trabectedin in the neoadjuvant setting for MRC liposarcoma was reported by Gronchi et al. [50]. In this multicenter phase II trial, 23 previously untreated patients received trabectedin prior to surgical resection. No disease progression was seen in any of the patients prior to surgery and $24 \%$ of patients experienced a RECIST objective response. Patients received a minimum of 3 and maximum of 6 c ycles, each lasting 3 weeks, prior to a 3 week was hout period after the last cycle leading up to surgery. Therefore, disease was monitored for a minimum of 3 months $(3 \times 3+3=12$ weeks $)$ in all study patients. Pathologic complete response was observed in $13 \%$ of patients as defined by complete absence of FUS-CHOP/DDIT3 translocation-positive tumor cells in the resection specimens. 
At the now standard dose of $1.5 \mathrm{mg} / \mathrm{m}^{2}$ given as a $24 \mathrm{~h}$ continuous infusion every 3 week s, established by Demetri et al. [46], trabectedin is overall fairly well tolerated [51]. Fatigue and nausea are the most common subjective complaints. Biochemical transaminitis without clinical manifestations is frequently seen and transient neutropenia is the major dose limiting toxicity, but occurs in only a minority of patients. Rare cases of fulminant hepatic failure and rhabdomyolysis have been reported.

Trabectedin was approved by the European Union in 2007 as second-line therapy for use in patients with soft tissue sarcoma and disease progression despite previous doxorubicin and ifosfamide treatment. In the United States, two phase III studies with trabectedin for advanced MRC liposarcoma are currently in progress (NCT01692678, NCT01343277).

Interestingly, another marine-derived compound, eribulin mesylate, was recently reported to also have selective activity in liposarcoma. In a phas e II study of four soft tissue sarcoma types, 37 patients (29\% of total) with adipocytic sarcoma treated with eribulin demonstrated a 3 month PFR of $47 \%$, the highest among the four cohorts [52]. PFR at 6 months was not reported. The majority (65\%) of the patients in the adipocytic sarcoma cohort actually had DD liposarcoma. Eribulin is a microtubule inhibitor but to our knowledge, a liposarcoma specific mechanism of action has not been described.

\subsection{MDM2 Antagonists}

In well differentiated (WD) and dedifferentiated (DD) liposarcomas, amplification of MDM2 is seen in virtually all tumors and in fact, is a reliable method for clinical diagnosis [2]. This observation and the knowledge of the important role of MDM2 as a negative regulator of p53, suggest that targeting MDM2 may be a promising approach to therapy, specifically for WD/DD liposarcoma. Preclinical studies using Nutlin-3A, a s elective MDM2 antagonist, restored p53 ac tivity in liposarcoma cells leading to preferential induction of cell cycle arrest and apoptosis [53,54].

Ray-Coquard et al. recently reported the first clinical trial of an MDM2 antagonist specifically in patients with WD and DD liposarcoma [55]. Twenty previously untreated patients from four centers in France were enrolled in a phase I, proof-of-mechanism study of the oral MDM2 antagonist, RG7112, given in the neoadjuvant setting. Analysis of resected tumors in these patients demonstrated restoration of $\mathrm{p} 53$ and downstream $\mathrm{p} 21$ expression as well as statistically significant reduction in Ki67-positive, proliferating tumor cells. A correlation was also noted between MDM2 antagonist treatment and increased numbers of TUNEL-positive, apoptotic tumor cells; however this failed to reach statistical significance. Six patients (30\%) experienced Grade 4 o ut of five neutropenia and three patients (15\%) had thrombocytopenia; almost all study patients experienced nausea, vomiting and fatigue. During the limited duration of neoadjuvant therapy (up to three 28-day cycles), the majority of patients (70\%) had stable disease and one patient had a partial response by RECIST criteria.

\subsection{CDK4 Antagonists}

Similar to MDM2, cyclin dependent kinase-4 or CDK4 is also consistently amplified in WD and DD liposarcoma and represents another appealing target for therapy for this histologic subtype. 
Mechanistically, CDK4 phosphorylates and functionally inactivates the retinoblastoma $(\mathrm{Rb})$ protein, which results in uninhibited cell cycle progression from G1 to S phase. CDK4 inhibition would thus restore native cell cycle regulation and prevent uncontrolled tumor cell proliferation.

Inhibitors of the broad family of CDKs have been tested for therapeutic value in both hematologic and solid tumors, with some compelling data for treatment efficacy in germ cell tumors and chronic lymphoid leukemia. One observation from these studies was that CDK-inhibitors may actually be effective as potentiators of cytotoxic chemotherapy agents. Luke et al. at the Memorial Sloan Kettering Cancer Center confirmed this concept in mouse xenograft models of soft tissue sarcoma, including DD liposarcoma, combining doxorubicin with the pan-CDK inhibitor, flavopiridol [56]. The authors then enrolled 31 patients with advanced soft tissue sarcoma in a phase I dose-escalation study of flavopiridol in combination with fixed dose doxorubicin. Fifteen patients ( $48 \%$ of total) had WD or DD liposarcoma and one patient had pleomorphic liposarcoma. Overall, toxicities were mostly hematologic (neutropenia, thrombocytopenia) and low to moderate grade; no maximum tolerated dose was reached. WD/DD liposarcoma patients demonstrated reasonable treatment efficacy. Among the 12 evaluable patients in this cohort, seven had stable disease at 12 weeks (PFR@3 mo=58\%) and 3 at 24 weeks (PFR@6 mo=25\%). One patient had stable disease for 99 weeks.

The first in-human study of PD 0332991, an oral CDK4/6 specific inhibitor, was reported by Schwartz et al. [57]. Patients were enrolled in this phase I study if they had either non-Hodgkin's lymphoma or Rb-positive advanced solid tumors including WD/DD liposarcoma. Dose-limiting hematologic toxicities were observed in six patients (18\%) for the entire cohort. Stable disease was observed in four out of seven patients with liposarcoma including one patient with a durable, toxicity-free response ( $>23$ cycles), despite previous progression on a tyrosine kinase receptor inhibitor. The authors have now opened a phase II study of PD 0332991, specifically for patients with Rb-positive liposarcoma (NCT01209598).

\subsection{PPAR-Gamma Agonists}

Peroxisome proliferator-activated receptors (PPAR) are critical regulators of normal adipocyte differentiation. PPAR-gamma is one of three isoforms that forms a heterodimeric complex with the retinoid $\mathrm{X}$ receptor to regulate transcription of adipocyte-specific genes involved in the terminal adipocyte differentiation pathway. In human liposarcoma cells, PPAR-gamma agonist not only induced adipocyte differentiation but demonstrated anti-tumor activity in vitro [58,59]. Activation of PPAR-gamma thus represents an attractive target particularly for DD, MRC and pleomorphic liposarcoma, as a mechanism to revert these subtypes to a more well differentiated phenotype with potentially more indolent disease progression, and for its direct anti-tumor activity.

Despite a plausible biologic basis, reported human studies using PPAR-gamma agonists for liposarcoma have thus far had mixed results with low numbers of enrolled patients. Demetri et al. reported a proof-of-mechanism study conducted at the Dana Farber Cancer Institute in three liposarcoma patients ( $2 \mathrm{MRC}, 1 \mathrm{pl}$ eomorphic), using the anti-diabetic thiazolidinedione drug, troglitazone [59]. Tumor biopsies and mass-spectroscopy imaging demonstrated histologic and biochemical differentiation with accumulation of lipid droplets. The authors reported favorable safety and tolerability, however no data on anti-tumor activity was provided. Debrock et al. 
subsequently reported results from a phase II trial of rosiglitazone in 12 patients with DD and MRC liposarcoma [60]. Histologic and biochemical proof-of-mechanism was negligible and no clinical response was seen with a mean time to disease progression of 6 months. More recently, Pishvaian et al. reported more encouraging results with a phas e I study of efatutazone, a n ewer generation PPAR-gamma agonist [61]. Five out 31 patients (16\%) enrolled had liposarcoma (subtypes not specified) and one patient with MRC had a durable partial response for 690 days while on therapy. In vitro studies suggest that inhibition (as opposed to activation) of the PPAR-delta isoform may have anti-proliferative effect specifically for liposarcoma [62].

\subsection{Nelfinavir}

Use of HIV protease inhibitors (PI) has been linked to a clinical syndrome of lipodystrophy in which treated patients demonstrate peripheral fat atrophy and central fat accumulation, along with insulin resistance and hyperlipidemia. Alterations in sterol regulatory element binding protein-1 (SREBP-1), a master transcriptional regulator of fatty acid and cholesterol synthesis, are thought to be the underlying mechanism for HIV PI lipodystrophy. Liposarcoma cells were shown to express SREBP-1 and investigators at the City of Hope Medical Center performed in vitro studies to show selective, dose-dependent anti-proliferative and pro-apoptotic activity of HIV PIs [63,64]. Among several HIV PIs tested, the most potent effects were seen with nelfinavir, which was subsequently chosen for clinical trial testing. Pan et al. recently reported results of a phase I trial of nelfinavir conducted in 20 patients with unresectable liposarcoma, 17 of whom had WD/DD, two MRC and one pleomorphic subtypes [65]. Although one patient had grade 3 pancreatitis, no other dose-limiting toxicities were seen. One patient with DD experienced a partial response for 14 months and four additional patients had stable disease. A phase II trial of nelfinavir in advanced liposarcoma was also conducted (NCT00233948) but the results have yet to be reported.

\subsection{Tyrosine Kinase Receptor Inhibitors}

Tyrosine kinase receptors (TKRs) are a diverse family of surface molecules recognized for their critical role in regulating multiple aspects of carcinogenesis, tumor cell proliferation and disease progression (e.g., angiogenesis, metastasis) across many solid tumor types. In the presence of a specific growth factor ligand, TKRs dimerize and initiate downstream, intracellular signaling pathways. In tumor cells, TKRs and their associated downstream molecules are frequently over-expressed or mutated, leading to constitutive activation or aberrant signaling.

Sleijfer et al. reported a large phase II study in patients with advanced soft tissue sarcoma, looking at pazopanib, an oral multi-targeted tyrosine kinase inhibitor with activity against VEGF, PDGF and KIT [66]. Sarcoma histology was diverse and 142 patients were included in the study, stratified by histology: liposarcoma, leiomyosarcoma, synovial sarcoma, and "other"; however, enrollment for patient with liposarcoma was stopped early because of lack of anti-tumor activity for this particular histology. Final analysis of 19 liposarcoma patients (subtypes not specified) demonstrated 3 month PFS in five out 19 of these patients (PFR $=26 \%$ ). To determine if any liposarcoma subtypes have any clinical benefit with pazopanib, another phase II study specific for advanced liposarcoma patients is currently open (NCT01506596). 
The Southwestern Oncology Group conducted a ph ase II study of sorafenib, a s imilar multitargeted anti-angiogenic TKR inhibitor in patients with vascular sarcomas, liposarcoma $(10$ patients = eight DD and two MRC) and leiomyosarcoma [67]. Von Mehren et al. reported that no tumor responses were seen in any of the patients. Consistent with the pazopanib trial data, patients with liposarcoma had the worst results with 3 month PFR of 30\%. Maki et al. reported a larger phase II trial of sorafenib in 145 patients with soft tissue sarcomas; however there were only three patients with liposarcoma, placed into the "other" category for analysis [68].

Sunitinib is another TKR that has similar targets as pazopanib and sorafenib and is also regarded as an anti-angiogenic agent. Interestingly, in a single institution study of 48 patients at the Moffitt Cancer Center, Tariq Mahmood et al. reported impressive efficacy with sunitinib specifically for liposarcoma [69]. Out of 17 evaluable patients with liposarcoma (subtypes not specified), 14 (=82\%) had stable disease). Three month PFR was $75 \%$ for untreated and $63 \%$ for previously treated liposarcoma patients, better results compared to the other histologies studied, leiomyosarcoma and UPS/MFH. Six month PFR was not reported. Sunitinib was well tolerated with Grade 1 or 2 hematologic and non-hematologic toxicities in the majority of patients. George et al. also reported a phase II trial of sunitinib in 53 pat ients with non-gastrointestinal stromal tumor sarcomas [70]. Only two patients had liposarcoma; one of whom had stable disease for 28 weeks.

It is unclear why sunitinib, but not pazopanib or sorafenib, has anti-tumor activity in liposarcoma despite similar molecular targets. The specific histologic subtype of liposarcoma patients enrolled (e.g., more indolent, WD liposarcoma), often not specified in the trials discussed, would have affected the interpretation of apparent drug efficacy. There may also be differential sensitivity to TKRs that is subtype-specific. Alternatively, in comparison to pazopanib or sorafenib, sunitinib may have selective affinity for other less well characterized TKRs targets that play an important role in tumor progression in liposarcoma. Recent work from our own group using an experimental in vitro and xenograft models of WD/DD liposarcoma has identified over-expression of several TKRs including EGFR, MET, AXL and IGFR, all of which may be potential targets [71].

\subsection{Immunotherapy}

Immunotherapy has recently gained much attention from success in large phase III trials with patients with metastatic melanoma, in which durable improvement in overall survival can be seen in patients that respond to treatment $[72,73]$. The immune system is capable of selectively recognizing and killing tumor cells with the theoretical advantage of minimal collateral damage to normal, non-malignant cells resulting in low toxicity for the patient [74]. Mechanistically, this is accomplished by natural or induced priming (e.g., vaccination) of cytotoxic CD8 T cells against surface antigens that are selectively expressed on tumor cells. Our group has reported preliminary studies to suggest that a natural immune response exists in a subset of WD liposarcoma that may be amenable to immunotherapeutic strategies used in metastatic melanoma [75].

In MRC liposarcoma, translational studies on paraffin-embedded tumors done independently by the Fred Hutchinson Cancer Center and Ohio State University have recently identified nearly uniform expression of NY-ESO-1 in virtually all (>90\%) of cases examined [76,77]. As a member of the highly-immunogenic cancer testis family antigens, NY-ESO-1 has been successfully used as the target for immunotherapy in a number of solid tumors, including synovial sarcoma [78]. Of note, 
NY-ESO is not expressed in WD/DD liposarcoma [79] and expression in pleomorphic liposarcoma has not been reported. At the Fred Hutchinson Cancer Center, a phase I trial of autologous NY-ESO-1 specific T cells in unresectable or metastatic MRC liposarcoma is currently open and recruiting participants (NCT01477021).

\subsection{Other Potential Novel Systemic Therapies from Preclinical Studies}

A number of other potential strategies for novel therapies in liposarcoma have not yet been tested, to our knowledge, in the setting of a clinical trial. Using large scale genomic analysis of multiple soft tissue sarcoma types, Barretina et al. identified YEATS4, a transcription factor involved in p53 regulation, as a pot ential target in WD/DD liposarcoma [80]. Knockdown of YEATS4 in liposarcoma cell lines resulted in better in vitro anti-proliferative effects compared to MDM2. The authors also identified a high frequency (18\%) of mutations in PI3K in MRC liposarcoma. Patients with either kinase or helical domain mutations had worse disease specific survival, although the numbers of such patients were small. The PI3K/Akt pathways may also play an important role in the sarcomagenesis of WD liposarcoma. Gutierrez et al. used a zebrafish model to show that constitutive expression of Akt 2 in mesenchymal progenitor cells resulted in development of adipocytic tumors that histologically resemble human WD liposarcoma [81]. In looking at patient samples, $27 \%$ of WD/DD liposarcoma cases examined demonstrated aberrant Akt activation. Small molecule inhibition of this pathway using BEZ235 demonstrated a d ose dependent decrease in human liposarcoma cell viability and an increase in cell cycle arrest and apoptosis. These results are supported by findings recently reported by Smith et al., in w hich use of rapamycin, a $\mathrm{PI} 3 \mathrm{~K} / \mathrm{AKt} / \mathrm{mTOR}$ inhibitor, induced terminal differentiation and had anti-proliferative effects in a mouse xenograft model of DD liposarcoma [82]. Several other interesting candidate targets for novel systemic therapies, including c-jun, JNK and others, have been reported are comprehensively reviewed elsewhere $[3,4]$.

\section{Discussion/Conclusions}

The reported results of recent clinical trials for novel systemic therapies in advanced liposarcoma are overall encouraging. With several novel therapies, disease stabilization is seen with relatively low toxicity. Trabectedin in MRC liposarcoma, flavopiridol in WD/DD liposarcoma, and sunitinib in subtype-unspecified liposarcoma appear to have fairly comparable or higher PFR than what has been reported for conventional cytotoxic chemotherapy. Objective responses are even seen in some patients, particularly with trabectedin in MRC liposarcoma. The caveat, of course, is that the current data is based on phase I and early phase II trials with relatively low numbers of patients (Table 2). More mature, phase II and III data in larger patient cohorts are clearly needed to make any definitive conclusions or alter current use of cytotoxic chemotherapy as first-line therapy.

For the majority of novel therapies, treatment efficacy is heavily dependent on histologic subtype. A better understanding of characteristic genetic and molecular aberrations for each histologic subtype, has led to many of the novel therapies discussed, which directly target these aberrations. Clear disparity, however, exists in the level of development of novel systemic therapies among the three liposarcoma subtypes. In contrast to MRC liposarcoma, which already has known sensitivity to 
radiation therapy [11] and conventional cytotoxic chemotherapy $[15,16]$ and now, trabectedin, novel therapies are poorly developed and have not been meaningfully evaluated in human studies for pleomorphic liposarcoma, the most aggressive histologic subtype. Barretina et al. reported that up to $17 \%$ of cases exhibit mutations in p53 [80]. More laboratory based studies are needed to better understand these mutations and how to best target them, as well as to identify other potential targets. For pleomorphic liposarcoma, this is of course challenging given its inherent genetic complexity and the overall rarity of this subtype.

Although clinical trials in liposarcoma should ideally be based on histologic subtype, sufficient enrollment of patients within a limited period of time can be very challenging. With the exception of trabectedin and eribulin, all of the other novel systemic therapies have been evaluated in trials with at most twenty patients (Table 2). We strongly advocate referral to specialized centers to consolidate patient distribution and expedite enrollment. Specialized centers have the resources and laboratory support needed to appropriately analyze tumor response and validate potential biomarkers. This is particularly important in neoadjuvant trials, which allow for molecular proof-of-mechanism by analysis of the resected tumor in treated patients. Specialized centers are also best poised to study tumors from patients with progression of disease while receiving trial therapy, which is equally critical to understanding tumor resistance and escape mechanisms. Ultimately, for larger scale trials (e.g., phase III), m ulti-institutional and even intercontinental group collaborations between specialized centers are needed.

In liposarcoma it is also important to define the pattern and extent of disease burden at the time of enrollment in a clinical trial. Even within the same histologic subtype, the broad category of "advanced disease" may include an isolated but unresectable tumor involving the root of the small bowel mesentery, intra-abdominal sarcomatosis, or distant visceral organ metastasis (e.g., lung) without locoregional recurrence; yet, each of these situations represents a unique disease biology with potentially different responses to therapy. Although it may not be practical to design a liposarcoma trial which enrolls only patients with a specific pattern or extent of disease, an attempt to meticulously document this may permit more meaningful subgroup analyses to determine which disease patterns may or may not respond to novel therapy. Recognition of lack of treatment efficacy for a specific disease pattern may also suggest exploration of alternative methods of drug delivery (e.g., local or regional therapy for the patient with a non-metastatic, but unresectable tumor).

Although all of the trials in this review focused on a single novel systemic therapy, combination approaches within a given histologic subtype should also be explored. Within each histologic subtype of liposarcoma, the multiple key genetic and molecular aberrations are often found together in the same tumor (e.g., MDM2 and CDK4 amplification in WD/DD liposarcoma). Therefore analogous to conventional cytotoxic chemotherapy, combinations of novel therapies may lead to synergistic effects that result in better disease stabilization and possibly, objective response. Combining molecular based therapy with immunotherapy may increase response rates in a durable fashion, leading to improved overall survival [83].

In conclusion, we feel that this is an exciting time for the development of biology-driven, novel systemic therapies for liposarcoma. The sheer abundance of clinical trials testing these biology-driven novel therapies truly exemplifies the "bench to bedside" translation of laboratory based findings to patient treatment, even for a relatively rare solid tumor. In recent years, several 
unique liposarcoma subtype-specific cell lines and experimental model systems have also been reported, which will certainly enhance preclinical testing of novel therapies [71,82,84-87]. With thoughtful design and conduct of clinical trials, we are hopeful that the near future will bring an emergence of several robust treatment options for the liposarcoma patient with advanced disease.

\section{References}

1. Clark, M.A.; Fisher, C.; Judson, I.; Thomas, J.M. Soft-tissue sarcomas in adults. N. Engl. J. Med. 2005, 353, 701-711.

2. Dodd, L.G. Update on liposarcoma: A review for cytopathologists. Diagn. Cytopathol. 2012, 40, 1122-1131.

3. Hoffman, A.; Lazar, A.J.; Pollock, R.E.; Lev, D. New frontiers in the treatment of liposarcoma, a t herapeutically resistant malignant cohort. Drug Resist. Updat. 2011, 14, $52-66$.

4. Conyers, R.; Young, S.; Thomas, D.M. Liposarcoma: Molecular genetics and therapeutics. Sarcoma 2011, doi:10.1155/2011/483154.

5. Singer, S.; Antonescu, C.R.; Riedel, E.; Brennan, M.F. Histologic subtype and margin of resection predict pattern of recurrence and survival for retroperitoneal liposarcoma. Ann. Surg. 2003, 238, 358-370.

6. Lahat, G.; Anaya, D.A.; Wang, X.; Tuvin, D.; Lev, D.; Pollock, R.E. Resectable well-differentiated versus dedifferentiated liposarcomas: Two different diseases possibly requiring different treatment approaches. Ann. Surg. Oncol. 2008, 15, 1585-1593.

7. Ghadimi, M.P.; Al-Zaid, T.; Madewell, J.; Peng, T.; Colombo, C.; Hoffman, A.; Creighton, C.J.; Zhang, Y.; Zhang, A.; Lazar, A.J.; et al. Diagnosis, management, and outcome of patients with dedifferentiated liposarcoma systemic metastasis. Ann. Surg. Oncol. 2011, 18, $3762-3770$.

8. Fiore, M.; Grosso, F.; Lo Vullo, S.; Pennacchioli, E.; Stacchiotti, S.; Ferrari, A.; Collini, P.; Lozza, L.; Mariani, L.; Casali, P.G.; et al. Myxoid/round cell and pleomorphic liposarcomas: Prognostic factors and survival in a series of patients treated at a single institution. Cancer 2007, 109, 2522-2531.

9. De Vreeze, R.S.; de Jong, D.; Tielen, I.H.; Ruijter, H.J.; Nederlof, P.M.; Haas, R.L.; van Coevorden, F. Primary retroperitoneal myxoid/round cell liposarcoma is a nonexisting disease: An immunohistochemical and molecular biological analysis. Mod. Pathol. 2009, 22, 223-231.

10. Schwab, J.H.; Boland, P.; Guo, T.; Brennan, M.F.; Singer, S.; Healey, J.H.; Antonescu, C.R. Skeletal metastases in myxoid liposarcoma: An unusual pattern of distant spread. Ann. Surg. Oncol. 2007, 14, 1507-1514.

11. Guadagnolo, B.A.; Zagars, G.K.; Ballo, M.T.; Patel, S.R.; Lewis, V.O.; Benjamin, R.S.; Pollock, R.E. Excellent local control rates and distinctive patterns of failure in myxoid liposarcoma treated with conservation surgery and radiotherapy. Int. J. Radiat. Oncol. Biol. Phys. 2008, 70, 760-765. 
12. Asano, N.; Susa, M.; Hosaka, S.; Nakayama, R.; Kobayashi, E.; Takeuchi, K.; Horiuchi, K.; Suzuki, Y.; Anazawa, U.; Mukai, M.; et al. Metastatic patterns of myxoid/round cell liposarcoma: A review of a 25 -year experience. Sarcoma 2012, 2012, 345161.

13. De Vreeze, R.; de J ong, D.; Nederlof, P.; Ruijter, H.J.; Boerrigter, L.; Haas, R.; van Coevorden, F. Multifocal myxoid liposarcoma-Metastasis or second primary tumor? A molecular biological analysis. J. Mol. Diagn. 2010, 12, 238-243.

14. Antonescu, C.R.; Elahi, A.; Healey, J.H.; Brennan, M.F.; Lui, M.Y.; Lewis, J.; Jhanwar, S.C.; Woodruff, J.M.; Ladanyi, M. Monoclonality of multifocal myxoid liposarcoma: Confirmation by analysis of TLS-CHOP or EWS-CHOP rearrangements. Clin. Cancer Res. 2000, 6, $2788-2793$.

15. Patel, S.R.; Burgess, M.A.; Plager, C.; Papadopoulos, N.E.; Linke, K.A.; Benjamin, R.S. Myxoid liposarcoma. Experience with chemotherapy. Cancer 1994, 74, 1265-1269.

16. Jones, R.L.; Fisher, C.; Al-Muderis, O.; Judson, I.R. Differential sensitivity of liposarcoma subtypes to chemotherapy. Eur. J. Cancer 2005, 41, 2853-2860.

17. Miettinen, M.; Enzinger, F.M. Epithelioid variant of pleomorphic liposarcoma: A study of 12 cases of a distinctive variant of high-grade liposarcoma. Mod. Pathol. 1999, 12, 722-728.

18. Hornick, J.L.; Bosenberg, M.W.; Mentzel, T.; McMenamin, M.E.; Oliveira, A.M.; Fletcher, C.D. Pleomorphic liposarcoma: Clinicopathologic analysis of 57 cases. Am. J. Surg. Pathol. 2004, 28, 1257-1267.

19. Ghadimi, M.P.; Liu, P.; Peng, T.; Bolshakov, S.; Young, E.D.; Torres, K.E.; Colombo, C.; Hoffman, A.; Broccoli, D.; Hornick, J.L.; et al. Pleomorphic liposarcoma: Clinical observations and molecular variables. Cancer 2011, 117, 5359-5369.

20. Coindre, J.M.; Mariani, O.; Chibon, F.; Mairal, A.; de Saint Aubain Somerhausen, N.; Favre-Guillevin, E.; Bui, N.B.; Stoeckle, E.; Hostein, I.; Aurias, A. Most malignant fibrous histiocytomas developed in the retroperitoneum are dedifferentiated liposarcomas: A review of 25 cases initially diagnosed as malignant fibrous histiocytoma. Mod. Pathol. 2003, 16, 256-262.

21. De Vreeze, R.S.; de J ong, D.; Nederlof, P.M.; Ariaens, A.; Tielen, I.H.; Frenken, L.; Haas, R.L.; van Coevorden, F. Added Value of Molecular Biological Analysis in Diagnosis and Clinical Management of Liposarcoma: A 30-Year Single-Institution Experience. Ann. Surg. Oncol. 2010, 17, 686-693.

22. Penel, N.; van Glabbeke, M.; Marreaud, S.; Ouali, M.; Blay, J.Y.; Hohenberger, P. Testing new regimens in patients with advanced soft tissue sarcoma: Analysis of publications from the last 10 years. Ann. Oncol. 2011, 22, 1266-1272.

23. Borden, E.C.; Amato, D.A.; Rosenbaum, C.; Enterline, H.T.; Shiraki, M.J.; Creech, R.H.; Lerner, H.J.; Carbone, P.P. Randomized comparison of three adriamycin regimens for metastatic soft tissue sarcomas. J. Clin. Oncol. 1987, 5, 840-850.

24. Bramwell, V.H.; Anderson, D.; Charette, M.L. Doxorubicin-based chemotherapy for the palliative treatment of adult patients with locally advanced or metastatic soft-tissue sarcoma: A meta-analysis and clinical practice guideline. Sarcoma 2000, 4, 103-112. 
25. Edmonson, J.H.; Ryan, L.M.; Blum, R.H.; Brooks, J.S.; Shiraki, M.; Frytak, S.; Parkinson, D.R. Randomized comparison of doxorubicin alone versus ifosfamide plus doxorubicin or mitomycin, doxorubicin, and cisplatin against advanced soft tissue sarcomas. J. Clin. Oncol. 1993, 11, 1269-1275.

26. Santoro, A.; Tursz, T.; Mouridsen, H.; Verweij, J.; Steward, W.; Somers, R.; Buesa, J.; Casali, P.; Spooner, D.; Rankin, E.; et al. Doxorubicin versus CYVADIC versus doxorubicin plus ifosfamide in first-line treatment of advanced soft tissue sarcomas: A randomized study of the European Organization for Research and Treatment of Cancer Soft Tissue and Bone Sarcoma Group. J. Clin. Oncol. 1995, 13, 1537-1545.

27. Le Cesne, A.; Judson, I.; Crowther, D.; Rodenhuis, S.; Keizer, H.J.; van Hoesel, Q.; Blay, J.Y.; Frisch, J.; van Glabbeke, M.; Hermans, C.; et al. Randomized phase III study comparing conventional-dose doxorubicin plus ifosfamide versus high-dose doxorubicin plus ifosfamide plus recombinant human granulocyte-macrophage colony-stimulating factor in advanced soft tissue sarcomas: A trial of the European Organization for Research and Treatment of Cancer/Soft Tissue and Bone Sarcoma Group. J. Clin. Oncol. 2000, 18, 2676-2684.

28. Van Oosterom, A.T.; Mouridsen, H.T.; Nielsen, O.S.; Dombernowsky, P.; Krzemieniecki, K.; Judson, I.; Svancarova, L.; Spooner, D.; Hermans, C.; van Glabbeke, M.; et al. EORTC Soft Tissue and Bone Sarcoma Group. Results of randomised studies of the EORTC Soft Tissue and Bone Sarcoma Group (STBSG) with two different ifosfamide regimens in first- and second-line chemotherapy in advanced soft tissue sarcoma patients. Eur. J. Cancer 2002, 38, 2397-2406.

29. Buesa, J.M.; Mouridsen, H.T.; van Oosterom, A.T.; Verweij, J.; Wagener, T.; Steward, W.; Poveda, A.; Vestlev, P.M.; Thomas, D.; Sylvester, R. High-dose DTIC in advanced soft-tissue sarcomas in the adult. A phase II study of the E.O.R.T.C. Soft Tissue and Bone Sarcoma Group. Ann. Oncol. 1991, 2, 307-309.

30. Antman, K.; Crowley, J.; Balcerzak, S.P.; Rivkin, S.E.; Weiss, G.R.; Elias, A.; Natale, R.B.; Cooper, R.M.; Barlogie, B.; Trump, D.L.; et al. An intergroup phase III randomized study of doxorubicin and dacarbazine with or without ifosfamide and mesna in advanced soft tissue and bone sarcomas. J. Clin. Oncol. 1993, 11, 1276-1285.

31. Maki, R.G.; Wathen, J.K.; Patel, S.R.; Priebat, D.A.; Okuno, S.H.; Samuels, B.; Fanucchi, M.; Harmon, D.C.; Schuetze, S.M.; Reinke, D.; Thall, P.F.; et al. Randomized phase II study of gemcitabine and docetaxel compared with gemcitabine alone in patients with metastatic soft tissue sarcomas: Results of sarcoma alliance for research through collaboration study 002 . J. Clin. Oncol. 2007, 25, 2755-2763.

32. Katz, D.; Boonsirikamchai, P.; Choi, H.; Lazar, A.J.; Wang, W.L.; Xiao, L.; Park, M.S.; Ravi, V.; Benjamin, R.S.; Araujo, D.M. Efficacy of first-line doxorubicin and ifosfamide in myxoid liposarcoma. Clin. Sarcoma Res. 2012, 2, 2.

33. Italiano, A.; Toulmonde, M.; Cioffi, A.; Penel, N.; Isambert, N.; Bompas, E.; Duffaud, F.; Patrikidou, A.; Lortal, B.; Le Cesne, A.; et al. Advanced well-differentiated/dedifferentiated liposarcomas: Role of chemotherapy and survival. Ann. Oncol. 2012, 23, 1601-1607.

34. Italiano, A.; Garbay, D.; Cioffi, A.; Maki, R.G.; Bui, B. Advanced pleomorphic liposarcomas: Clinical outcome and impact of chemotherapy. Ann. Oncol. 2012, 23, 2205-2206. 
35. Jelić, S.; Kovcin, V.; Milanović, N.; Babović, N.; Kreacić, M.; Ristović, Z.; Vlajić, M.; Filipović-Ljesković, I. Randomised study of high-dose epirubicin versus high-dose epirubicin-cisplatin chemotherapy for advanced soft tissue sarcoma. Eur. J. Cancer 1997, 33, 220-225.

36. Hartmann, J.T.; Oechsle, K.; Huober, J.; Jakob, A.; Azemar, M.; Horger, M.; Kanz, L.; Bokemeyer, C. An open label, non-comparative phase II study of gemcitabine as salvage treatment for patients with pretreated adult type soft tissue sarcoma. Invest. New Drugs 2006, 24, 249-253.

37. Reichardt, P.; Oechsle, K.; Pink, D.; Bokemeyer, C.; Schneller, F.; Issels, R.; Kanz, L.; Hartmann, J.T. An open label, non-comparative phase II study of topotecan as salvage treatment for patients with soft tissue sarcoma. Invest. New Drugs 2003, 21, 481-486.

38. Van Glabbeke, M.; Verweij, J.; Judson, I.; Nielsen, O.S. EORTC Soft Tissue and Bone Sarcoma Group. Progression-free rate as the principal end-point for phase II trials in soft-tissue sarcomas. Eur. J. Cancer 2002, 38, 543-549.

39. Molinski, T.F.; Dalisay, D.S.; Lievens, S.L.; Saludes, J.P. Drug development from marine natural products. Nat. Rev. Drug Discov. 2009, 8, 69-85.

40. Forni, C.; Minuzzo, M.; Virdis, E.; Tamborini, E.; Simone, M.; Tavecchio, M.; Erba, E.; Grosso, F.; Gronchi, A.; Aman, P.; et al. Trabectedin (ET-743) promotes differentiation in myxoid liposarcoma tumors. Mol. Cancer Ther. 2009, 8, 449-457.

41. Germano, G.; Frapolli, R.; Simone, M.; Tavecchio, M.; Erba, E.; Pesce, S.; Pasqualini, F.; Grosso, F.; Sanfilippo, R.; Casali, P.G.; et al. Antitumor and anti-inflammatory effects of trabectedin on human myxoid liposarcoma cells. Cancer Res. 2010, 70, 2235-2244.

42. Germano, G.; Frapolli, R.; Belgiovine, C.; Anselmo, A.; Pesce, S.; Liguori, M.; Erba, E.; Uboldi, S.; Zucchetti, M.; Pasqualini, F.; et al. Role of macrophage targeting in the antitumor activity of trabectedin. Cancer Cell 2013, 23, 249-262.

43. Garcia-Carbonero, R.; Supko, J.G.; Manola, J.; Seiden, M.V.; Harmon, D.; Ryan, D.P.; Quigley, M.T.; Merriam, P.; Canniff, J.; Goss, G.; et al. Phase II and pharmacokinetic study of ecteinascidin 743 in patients with progressive sarcomas of soft tissues refractory to chemotherapy. J. Clin. Oncol. 2004, 22, 1480-1490.

44. Yovine, A.; Riofrio, M.; Blay, J.Y.; Brain, E.; Alexandre, J.; Kahatt, C.; Taamma, A.; Jimeno, J.; Martin, C.; Salhi, Y.; et al. Phase II study of ecteinascidin-743 in advanced pretreated soft tissue sarcoma patients. J. Clin. Oncol. 2004, 22, 890-899.

45. Le Cesne, A.; Blay, J.Y.; Judson, I.; van Oosterom, A.; Verweij, J.; Radford, J.; Lorigan, P.; Rodenhuis, S.; Ray-Coquard, I.; Bonvalot, S.; et al. Phase II study of ET-743 in advanced soft tissue sarcomas: A European Organisation for the Research and Treatment of Cancer (EORTC) soft tissue and bone sarcoma group trial. J. Clin. Oncol. 2005, 23, 576-584.

46. Demetri, G.D.; Chawla, S.P.; von Mehren, M.; Ritch, P.; Baker, L.H.; Blay, J.Y.; Hande, K.R.; Keohan, M.L.; Samuels, B.L.; Schuetze, S.; et al. Efficacy and safety of trabectedin in patients with advanced or metastatic liposarcoma or leiomyosarcoma after failure of prior anthracyclines and ifosfamide: Results of a $\mathrm{r}$ andomized phase II study of two different schedules. J. Clin. Oncol. 2009, 27, 4188-4196. 
47. Samuels, B.L.; Chawla, S.; Patel, S.; von Mehren, M.; Hamm, J.; Kaiser, P.E.; Schuetze, S.; Li, J.; Aymes, A.; Demetri, G.D. Clinical outcomes and safety with trabectedin therapy in patients with advanced soft tissue sarcomas following failure of prior chemotherapy: Results of a worldwide expanded access program study. Ann. Oncol. 2013, doi:10.1093/annonc/mds659.

48. Grosso, F.; Jones, R.L.; Demetri, G.D.; Judson, I.R.; Blay, J.Y.; Le Cesne, A.; Sanfilippo, R.; Casieri, P.; Collini, P.; Dileo, P.; et al. Efficacy of trabectedin (ecteinascidin-743) in advanced pretreated myxoid liposarcomas: A retrospective study. Lancet Oncol. 2007, 8, 595-602.

49. Grosso, F.; Sanfilippo, R.; Virdis, E.; Piovesan, C.; Collini, P.; Dileo, P.; Morosi, C.; Tercero, J.C.; Jimeno, J.; D'Incalci, M.; et al. Trabectedin in myxo id liposarcomas (MLS): A long-term analysis of a single-institution series. Ann. Oncol. 2009, 20, 1439-1444.

50. Gronchi, A.; Bui, B.N.; Bonvalot, S.; Pilotti, S.; Ferrari, S.; Hohenberger, P.; Hohl, R.J.; Demetri, G.D.; Le Cesne, A.; Lardelli, P.; et al. Phase II clinical trial of neoadjuvant trabectedin in patients with advanced localized myxoid liposarcoma. Ann. Oncol. 2012, 23, $771-776$.

51. Casali, P.G.; Sanfilippo, R.; D’Incalci, M. Trabectedin therapy for sarcomas. Curr. Opin. Oncol. 2010, 22, 342-346.

52. Schöffski, P.; Ray-Coquard, I.L.; Cioffi, A.; Bui, N.B.; Bauer, S.; Hartmann, J.T.; Krarup-Hansen, A.; Grünwald, V.; Sciot, R.; Dumez, H.; et al. European Organisation for Research and Treatment of Cancer (EORTC) Soft Tissue and Bone Sarcoma Group (STBSG). Activity of eribulin mesylate in patients with soft-tissue sarcoma: A phase $2 \mathrm{~s}$ tudy in four independent histological subtypes. Lancet Oncol. 2011, 12, 1045-1052.

53. Müller, C.R.; Paulsen, E.B.; Noordhuis, P.; Pedeutour, F.; Saeter, G.; Myklebost, O. Potential for treatment of liposarcomas with the MDM2 antagonist Nutlin-3A. Int. J. Cancer 2007, 121, 199-205.

54. Singer, S.; Socci, N.D.; Ambrosini, G.; Sambol, E.; Decarolis, P.; Wu, Y.; O'Connor, R.; Maki, R.; Viale, A.; Sander, C.; et al. Gene expression profiling of liposarcoma identifies distinct biological types/subtypes and potential therapeutic targets in well-differentiated and dedifferentiated liposarcoma. Cancer Res. 2007, 67, 6626-6636.

55. Ray-Coquard, I.; Blay, J.Y.; Italiano, A.; Le Cesne, A.; Penel, N.; Zhi, J.; Heil, F.; Rueger, R.; Graves, B.; Ding, M.; et al. Effect of the MDM2 antagonist RG7112 on the P53 pathway in patients with MDM2-amplified, well-differentiated or dedifferentiated liposarcoma: An exploratory proof-of-mechanism study. Lancet Oncol. 2012, 13, 1133-1140.

56. Luke, J.J.; D’Adamo, D.R.; Dickson, M.A.; Keohan, M.L.; Carvajal, R.D.; Maki, R.G.; de Stanchina, E.; Musi, E.; Singer, S.; Schwartz, G.K. The cyclin-dependent kinase inhibitor flavopiridol potentiates doxorubicin efficacy in advanced sarcomas: Preclinical investigations and results of a phase I dose-escalation clinical trial. Clin. Cancer Res. 2012, 18, 2638-2647.

57. Schwartz, G.K.; LoRusso, P.M.; Dickson, M.A.; Randolph, S.S.; Shaik, M.N.; Wilner, K.D.; Courtney, R.; O’Dwyer, P.J. Phase I study of PD 0332991, a cyclin-dependent kinase inhibitor, administered in 3-week cycles (Schedule 2/1). Br. J. Cancer 2011, 104, 1862-1868. 
58. Tontonoz, P.; Singer, S.; Forman, B.M.; Sarraf, P.; Fletcher, J.A.; Fletcher, C.D.; Brun, R.P.; Mueller, E.; Altiok, S.; Oppenheim, H.; et al. Terminal differentiation of human liposarcoma cells induced by ligands for peroxisome proliferator-activated receptor gamma and the retinoid X receptor. Proc. Natl. Acad. Sci. USA 1997, 94, 237-241.

59. Demetri, G.D.; Fletcher, C.D.; Mueller, E.; Sarraf, P.; Naujoks, R.; Campbell, N.; Spiegelman, B.M.; Singer, S. Induction of solid tumor differentiation by the peroxisome proliferator-activated receptor-gamma ligand troglitazone in patients with liposarcoma. Proc. Natl. Acad. Sci. USA 1999, 96, 3951-3956.

60. Debrock, G.; Vanhentenrijk, V.; Sciot, R.; Debiec-Rychter, M.; Oyen, R.; van Oosterom, A. A phase II trial with rosiglitazone in liposarcoma patients. Br. J. Cancer 2003, 89, 1409-1412.

61. Pishvaian, M.J.; Marshall, J.L.; Wagner, A.J.; Hwang, J.J.; Malik, S.; Cotarla, I.; Deeken, J.F.; He, A.R.; Daniel, H.; Halim, A.B.; et al. A phase 1 study of efatutazone, an oral peroxisome proliferator-activated receptor gamma agonist, administered to patients with advanced malignancies. Cancer 2012, 118, 5403-5413.

62. Wagner, K.D.; Benchetrit, M.; Bianchini, L.; Michiels, J.F.; Wagner, N. Peroxisome proliferator-activated receptor $\beta / \delta(\mathrm{PPAR} \beta / \delta)$ is highly expressed in liposarcoma and promotes migration and proliferation. J. Pathol. 2011, 224, 575-588.

63. Chow, W.A.; Guo, S.; Valdes-Albini, F. Nelfinavir induces liposarcoma apoptosis and cell cycle arrest by upregulating sterol regulatory element binding protein-1. Anticancer Drugs 2006, 17, 891-903.

64. Guan, M.; Fousek, K.; Jiang, C.; Guo, S.; Synold, T.; Xi, B.; Shih, C.C.; Chow, W.A. Nelfinavir induces liposarcoma apoptosis through inhibition of regulated intramembrane proteolysis of SREBP-1 and ATF6. Clin. Cancer Res. 2011, 17, 1796-1806.

65. Pan, J.; Mott, M.; Xi, B.; Hepner, E.; Guan, M.; Fousek, K.; Magnusson, R.; Tinsley, R.; Valdes, F.; Frankel, P.; et al. Phase I study of nelfinavir in liposarcoma. Cancer Chemother. Pharmacol. 2012, 70, 791-799.

66. Sleijfer, S.; Ray-Coquard, I.; Papai, Z.; Le Cesne, A.; Scurr, M.; Schöffski, P.; Collin, F.; Pandite, L.; Marreaud, S.; de Brauwer, A.; et al. Pazopanib, a m ultikinase angiogenesis inhibitor, in patients with relapsed or refractory advanced soft tissue sarcoma: A phase II study from the European organisation for research and treatment of cancer-soft tissue and bone sarcoma group (EORTC study 62043). J. Clin. Oncol. 2009, 27, 3126-3132.

67. Von Mehren, M.; Rankin, C.; Goldblum, J.R.; Demetri, G.D.; Bramwell, V.; Ryan, C.W.; Borden, E. Phase 2 Southwest Oncology Group-directed intergroup trial (S0505) of sorafenib in advanced soft tissue sarcomas. Cancer 2012, 118, 770-776.

68. Maki, R.G.; D’Adamo, D.R.; Keohan, M.L.; Saulle, M.; Schuetze, S.M.; Undevia, S.D.; Livingston, M.B.; Cooney, M.M.; Hensley, M.L.; Mita, M.M.; et al. Phase II study of sorafenib in patients with metastatic or recurrent sarcomas. J. Clin. Oncol. 2009, 27, 3133-3140. 
69. Tariq Mahmood, S.; Agresta, S.; Vigil, C.E.; Zhao, X.; Han, G.; D’Amato, G.; Calitri, C.E.; Dean, M.; Garrett, C.; Schell, M.J.; et al. Phase II study of sunitinib malate, a multitargeted tyrosine kinase inhibitor in patients with relapsed or refractory soft tissue sarcomas. Focus on three prevalent histologies: Leiomyosarcoma, liposarcoma and malignant fibrous histiocytoma. Int. J. Cancer 2011, 129, 1963-1969.

70. George, S.; Merriam, P.; Maki, R.G.; van den Abbeele, A.D.; Yap, J.T.; Akhurst, T.; Harmon, D.C.; Bhuchar, G.; O’Mara, M.M.; D’Adamo, D.R.; et al. Multicenter phase II trial of sunitinib in the treatment of nongastrointestinal stromal tumor sarcomas. J. Clin. Oncol. 2009, 27, 3154-3160.

71. Peng, T.; Zhang, P.; Liu, J.; Nguyen, T.; Bolshakov, S.; Belousov, R.; Young, E.D.; Wang, X.; Brewer, K.; López-Terrada, D.H.; et al. An ex perimental model for the study of well-differentiated and dedifferentiated liposarcoma; deregulation of targetable tyrosine kinase receptors. Lab. Invest. 2011, 91, 392-403.

72. Hodi, F.S.; O’Day, S.J.; McDermott, D.F.; Weber, R.W.; Sosman, J.A.; Haanen, J.B.; Gonzalez, R.; Robert, C.; Schadendorf, D.; Hassel, J.C.; et al. Improved survival with ipilimumab in patients with metastatic melanoma. N. Engl. J. Med. 2010, 363, 711-723.

73. Robert, C.; Thomas, L.; Bondarenko, I.; O’Day, S.; Weber, J.; Garbe, C.; Lebbe, C.; Baurain, J.F.; Testori, A.; Grob, J.J.; Davidson, N.; et al. Ipilimumab plus dacarbazine for previously untreated metastatic melanoma. N. Engl. J. Med. 2011, 364, 2517-2526.

74. Pollack, S.M.; Loggers, E.T.; Rodler, E.T.; Yee, C.; Jones, R.L. Immune-based therapies for sarcoma. Sarcoma 2011, 2011, 438940.

75. Tseng, W.W.; Demicco, E.G.; Lazar, A.J.; Lev, D.C.; Pollock, R.E. Lymphocyte composition and distribution in inflammatory, well-differentiated retroperitoneal liposarcoma: Clues to a potential adaptive immune response and therapeutic implications. Am. J. Surg. Pathol. 2012, 36, 941-944.

76. Pollack, S.M.; Jungbluth, A.A.; Hoch, B.L.; Farrar, E.A.; Bleakley, M.; Schneider, D.J.; Loggers, E.T.; Rodler, E.; Eary, J.F.; Conrad, E.U., 3rd; et al. NY-ESO-1 is a ubiquitous immunotherapeutic target antigen for patients with myxoid/round cell liposarcoma. Cancer 2012, 118, 4564-4570.

77. Hemminger, J.A.; Ewart Toland, A.; Scharschmidt, T.J.; Mayerson, J.L.; Kraybill, W.G.; Guttridge, D.C.; Iwenofu, O.H. The cancer-testis antigen NY-ESO-1 is highly expressed in myxoid and round cell subset of liposarcomas. Mod. Pathol. 2013, 26, 282-288.

78. Lai, J.P.; Robbins, P.F.; Raffeld, M.; Aung, P.P.; Tsokos, M.; Rosenberg, S.A.; Miettinen, M.M.; Lee, C.C. NY-ESO-1 expression in synovial sarcoma and other mesenchymal tumors: Significance for NY-ESO-1-based targeted therapy and differential diagnosis. Mod. Pathol. 2012, 25, 854-858.

79. Pollack, S. Personal communication. Fred Hutchinson Cancer Center, Seattle, WA, USA, 2013.

80. Barretina, J.; Taylor, B.S.; Banerji, S.; Ramos, A.H.; Lagos-Quintana, M.; Decarolis, P.L.; Shah, K.; Socci, N.D.; Weir, B.A.; Ho, A.; et al. Subtype-specific genomic alterations define new targets for soft-tissue sarcoma therapy. Nat. Genet. 2010, 42, 715-721. 
81. Gutierrez, A.; Snyder, E.L.; Marino-Enriquez, A.; Zhang, Y.X.; Sioletic, S.; Kozakewich, E.; Grebliunaite, R.; Ou, W.B.; Sicinska, E.; Raut, C.P.; Demetri, G.D.; et al. Aberrant AKT activation drives well-differentiated liposarcoma. Proc. Natl. Acad. Sci. USA 2011, 108, 16386-16391.

82. Smith, K.B.; Tran, L.M.; Tam, B.M.; Shurell, E.M.; Li, Y.; Braas, D.; Tap, W.D.; Christofk, H.R.; Dry, S.M.; Eilber, F.C.; et al. Novel Dedifferentiated Liposarcoma Xenograft Models Reveal PTEN Down-Regulation as a Malignant Signature and Response to PI3K Pathway Inhibition. Am. J. Pathol. 2013, 182, 1400-1411.

83. Sharma, P.; Wagner, K.; Wolchok, J.D.; Allison, J.P. Novel cancer immunotherapy agents with survival benefit: Recent successes and next steps. Nat. Rev. Cancer 2011, 11, 805-812.

84. Pedeutour, F.; Maire, G.; Pierron, A.; Thomas, D.M.; Garsed, D.W.; Bianchini, L.; Duranton-Tanneur, V.; Cortes-Maurel, A.; Italiano, A.; Squire, J.A.; et al. A newly characterized human well-differentiated liposarcoma cell line contains amplifications of the 12q12-21 and 10p11-14 regions. Virchows Arch. 2012, 461, 67-78.

85. Ariizumi, T.; Ogose, A.; Kawashima, H.; Hotta, T.; Li, G.; Xu, Y.; Hirose, T.; Endo, N. Establishment and characterization of a novel dedifferentiated liposarcoma cell line, NDDLS-1. Pathol. Int. 2011, 61, 461-468.

86. Uboldi, S.; Bernasconi, S.; Romano, M.; Marchini, S.; Fuso Nerini, I.; Damia, G.; Ganzinelli, M.; Marangon, E.; Sala, F.; Clivio, L.; et al. Characterization of a new trabectedin-resistant myxoid liposarcoma cell line that shows collateral sensitivity to methylating agents. Int. J. Cancer 2012, 131, 59-69.

87. Stratford, E.W.; Castro, R.; Daffinrud, J.; Skårn, M.; Lauvrak, S.; Munthe, E.; Myklebost, O. Characterization of liposarcoma cell lines for preclinical and biological studies. Sarcoma 2012, 2012, 148614. 
Reprinted from Cancers. Cite as: Verschraegen, C.F.; Movva, S.; Ji, Y.; Schmit, B.; Quinn, R.H.; Liem, B.; Bocklage, T.; Shaheen, M. A Phase I Study of the Combination of Temsirolimus with Irinotecan for Metastatic Sarcoma. Cancers 2013, 5, 418-429.

Article

\section{A Phase I Study of the Combination of Temsirolimus with Irinotecan for Metastatic Sarcoma}

Claire F. Verschraegen ${ }^{1}{ }^{1}$, Sujana Movva ${ }^{2}$, Yongli Ji ${ }^{1}$, Berndt Schmit ${ }^{3}$, Robert H. Quinn ${ }^{4}$, Ben Liem ${ }^{5}$, Therese Bocklage ${ }^{6}$ and Monte Shaheen ${ }^{7}$

1 Department of Hematology/Oncology, The University of Vermont Cancer Center, 89 Beaumont Ave., Burlington, VT 05405, USA

2 Department of Medical Oncology, Fox Chase Cancer Center, Pennsylvania, PA 19111, USA

3 Department of Radiology, University of Pittsburgh Medical Center East, Pittsburgh, PA 15146, USA

4 Department of Orthopaedics, University of Texas Health Science Center San Antonio, San Antonio, TX 78229, USA

5 Department of Radiation Oncology, University of New Mexico Cancer Center, Albuquerque, NM 87131, USA

6 Department of Pathology, University of New Mexico Cancer Center, Albuquerque, NM 87131, USA

7 Division of Hematology/Oncology, University of New Mexico Cancer Center, Albuquerque, NM 87131, USA

* Author to whom correspondence should be addressed; E-Mail:

claire.verschraegen@vtmednet.org; Tel.: +1-802-656-5487; Fax: +1-802-656-5414.

Received: 16 February 2013; in revised form: 25 March 2013 /Accepted: 6 April 2013 /

Published: 11 April 2013

Abstract: mTOR inhibitors are emerging as important anti-neoplastic agents with a wide
range of clinical applications. The topoisomerase I inhibitor irinotecan is a potent DNA
damaging drug, with a b road spectrum of anticancer activities. mTOR appears to
enhance cancer cell survival following DNA damage, thus the inhibition of mTOR after
irinotecan could theoretically show synergistic activities in patients. Both mTOR
inhibitors and irinotecan have been used as single agents in soft tissue sarcomas with
limited efficacy. We completed a phase I trial of the combination of the mTOR inhibitor,
temsirolimus, and irinotecan in patients with advanced soft tissue sarcoma. Seventeen
patients were recruited. The Phase II recommended dose is 20 mg of temsirolimus and 80
$\mathrm{mg} / \mathrm{m}^{2}$ of irinotecan administered on weekly basis for three out of four weeks. Most 
frequently encountered toxicities include cytopenias, fatigue, and gastrointestinal toxicities. Two patients (one with leiomyosarcoma and one with high grade undifferentiated sarcoma) had stable disease for more than 12 months.

Keywords: sarcoma; mTOR inhibitors; irinotecan; temsirolimus; phase I

\section{Introduction}

The mammalian target of rapamycin (mTOR) is an essential signaling protein that has an important role in various cellular processes, particularly in nutrient sensing, cell mass regulation and proliferation [1]. mTOR operates downstream from the PI3K signaling pathway, which is frequently up-regulated in cancer [2]. mTOR is an atypical serine/threonine protein kinase of the phosphatidylinositol 3-kinase (PI3K)-related kinase family, which functions by complexing with other proteins (mTOR complex 1 and 2) to promote protein translation through phosphorylation of the translational regulators, eukaryotic translation initiation factor 4E (eIF4E)-binding protein 1 (4E-BP1) and S6 k inase 1 (S6K1) (Figure 1) [1,3]. mTOR also up-regulates the expression of hypoxia inducible factor (HIF)-1 $\alpha$, which mediates the expression of several key angiogenic factors including the vascular endothelial growth factor. Thus, inhibition of mTOR also results in an antiangiogenic effect in sarcomas [4,5]. Additionally, the loss of p53, a common event in cancer, promotes mTOR activation [6]. Multiple familial cancer syndromes occur due to mutations in genes encoding proteins that signal upstream of the mTOR complexes, (including tuberous sclerosis proteins 1 and 2 (TSC1/2), serine threonine kinase 11 (STK11 or Lkb1), phosphatase and tensin homolog (PTEN), and neurofibromatosis type 1 (NF1). Oncogenic activation of mTOR induces several processes required for cancer cell growth, survival, and proliferation [1]. Thus, inhibition of mTOR kinase activity impacts multiple pathways that are important for tumor maintenance such as the protein translation of several oncogenes [3].

Two mTOR inhibitors, analogs of rapamycin, temsirolimus (Torisel ${ }^{\circledR}$ ) and everolimus (RAD-001, Afinitor $^{\circledR}$ ) are clinically available for renal cell cancer [7-9], well differentiated neuroendocrine tumors [10], and in combination with hormonal therapy for metastatic breast cancer [11]. mTOR inhibitors have modest inhibitory activity against soft tissue sarcoma except in perivascular epithelioid cell tumors [12] and angiomyolipomas [13]. In a ph ase II study of temsirolimus in patients with untreated advanced soft tissue sarcoma, two patients (with undifferentiated fibrosarcoma and uterine leiomyosarcoma) achieved a confirmed partial response lasting 3 and 17 months, respectively [14]. A phase II study of ridaforolimus in patients with refractory sarcoma also showed a potential clinical benefit [15]. Ridaforolimus, as maintenance therapy after chemotherapy, was tested in a phase III trial in patients with soft tissue sarcoma. A clinical benefit rate of $29 \%$ was observed in the 212 evaluable patients with a minor survival advantage [16]. Most common adverse events are anemia, hyperglycemia, dyspnea, nausea and vomiting, neutropenia, hypokalemia, fatigue and stomatitis [17].

Irinotecan is a topoisomerase I inhibitor that generates DNA damage in replicating cells [18], and triggers cell cycle arrest, apoptosis, and senescence [19]. Single agent and various combinations of 
irinotecan have been used as salvage therapies for a variety of pediatric soft tissue sarcomas with responses varying from $15 \%$ to $50 \%$ [20-23]. The pharmacokinetics of topoisomerase inhibitors are highly variable, thus not reliable and not helpful for defining the optimal schedule. Animal experimentation shows that prolonged exposure is better than high doses intermittently, thus the choice of a weekly dosing for this study [24].

Figure 1. mTOR pathway.

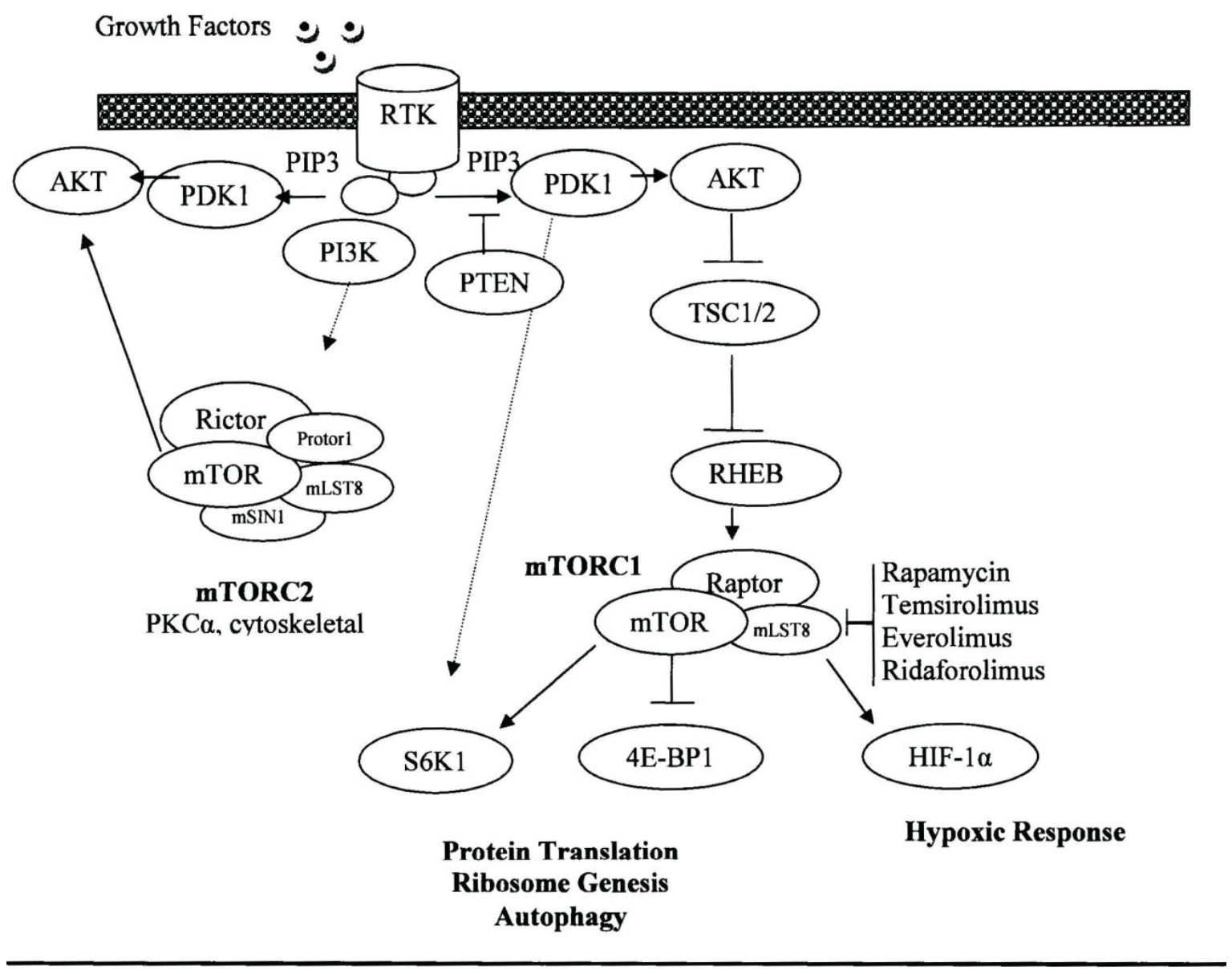

Cancer cell survival following irinotecan-induced DNA damage is regulated by mTOR. A synergy was found between irinotecan and rapamycin in human xenografts colon cancers. mTOR interferes with hypoxia-inducible factor- $1 \alpha(\mathrm{HIF}-1 \alpha)$, a key transcription factor with a pivotal role in tumor cell metabolism (Figure 1). Topoisomerase I inhibitors, such as irinotecan, also inhibits the accumulation of HIF-1 $\alpha$. Human colon cancers xenografted in nude mice treated with the combination of low doses irinotecan and rapamycin showed a potent inhibition of the mTOR/HIF-1 $\alpha$ axis, which was accompanied by a dramatic reduction in tumor volume, compared to single agent. In vitro experiments further confirmed this synergy [25]. These results identify HIF-1 $\alpha$ as the potential target and provide a rationale for this study testing the combination of irinotecan and temsirolimus in soft tissue sarcomas. We hypothesized that mTOR inhibition could potentiate the clinical activity of irinotecan by preventing the regulation of cancer cell survival. The primary objective of this study was to determine the maximum tolerated dose (MTD) and the toxicity profile of the combination. A secondary objective was to evaluate the clinical activity of this regimen. 


\section{Experimental Section}

The study (NCT00996346) was approved by the Institutional Review Board and all patients signed an informed consent before enrollment. This trial was conducted according to Good Clinical Practice guidelines. Eligibility criteria included patients older than 17 years, with a histologically proven advanced soft tissue sarcoma, and who had failed at least one prior treatment for metastatic disease. Recurrence had to be assessable by RECIST criteria version 1. Patients had to have a performance status of 0-2 and normal organ function (peripheral granulocyte count of equal or greater than 1,500 cells $/ \mathrm{mm}^{3}$, hemoglobin equal or greater than $8 \mathrm{~g} / \mathrm{dL}$, platelet count equal or greater than $100,000 / \mathrm{mm}^{3}$ and absence of a regular red blood cell transfusion requirement; normal hepatic function with a total bilirubin equal or lower than the upper limit of normal and SGOT or SGPT equal or lower than two times the upper limit of normal, adequate renal function defined by a serum creatinine equal or lower than 1.5 times the upper limit of normal, and a clinically normal cardiac function) with a fasting total cholesterol and triglyceride levels below $350 \mathrm{mg} / \mathrm{dL}$ and $400 \mathrm{mg} / \mathrm{dL}$, respectively. Pregnant women or nursing mothers were not eligible.

The study was planned to be conducted in two parts. A 3+3 phase I two-arm crossing design was utilized in Part 1 to define the MTD [26]. A part 2 of the study (Expansion phase) was planned if two or more objective responses were observed during the part 1 after recruiting 17 patients, but was never implemented. Part 1 was done in two arms using a fixed dose of one drug in each arm [26]. Both drugs were administered on a weekly basis for three consecutive doses, followed by one week of rest. Irinotecan was given first over $60 \mathrm{~min}$, followed by temsirolimus over $30 \mathrm{~min}$. Each cycle lasted four weeks. No intra-patient dose escalations were allowed. Each patient was treated until disease progression or intolerable side effects developed. Arm A consisted of a fixed dose of irinotecan at $80 \mathrm{mg} / \mathrm{m}^{2}$ and a starting dose of temsirolimus of $15 \mathrm{mg}$ with increments of $5 \mathrm{mg}$ for subsequent cohorts. Arm B consisted of a fixed dose of temsirolimus $25 \mathrm{mg}$ and a starting dose of irinotecan of $50 \mathrm{mg} / \mathrm{m}^{2}$ with increments of $15 \mathrm{mg} / \mathrm{m}^{2}$ for subsequent cohorts.

DLTs were defined as grade 3 neutropenia on retreatment day, a grade 4 febrile neutropenia, a drug-related grade 3 or 4 non-hematologic toxicity (except fatigue, nausea, vomiting or grade 3 hypersensitivity reaction), a grade 2 or greater motor or sensory neuropathy, or inability to receive consecutive doses of treatment during the first four weeks of treatment. Adverse events were assessed with the NCI Common Terminology Criteria for Adverse Events, Version 3. The MTD was defined as the dose preceding that at which at least two out of six patients experience a DLT. For patients' safety, doses of the fixed drug could be reduced by one tier in subsequent cycles, after the first cycle was appropriately completed. Statistics were descriptive for part 1 . Repeated cycles at the same dose level were given to patients who benefited from treatment (complete or partial remission, or stabilization of disease) after resolution of non-hematologic toxicity to a grade 0 or 1 , and return of absolute neutrophil count to $\geq 1,500$ cells $/ \mathrm{mm}^{3}$ and platelet count to $\geq 100,000 / \mathrm{mm}^{3}$. If toxic effects precluding therapy persisted for three weeks or more, patients were removed from the study. Patients were examined every four weeks and blood parameters were verified weekly. Imaging studies were repeated every eight weeks and tumors evaluated by the RECIST criteria version 1 . The clinical benefit rate was calculated as the sum of complete and partial responses plus stable disease. 


\section{Results and Discussion}

Thirty-five patients were screened and 17 patients were enrolled between October 2009 and May 2011. Reasons for enrollment failure were ineligibility (16 patients) and refusal to participate (two patients). Patient characteristics are described in Table 1.

Table 1. Patient characteristics $(\mathrm{N}=17)$.

\begin{tabular}{ccc}
\hline Median Age & & 57 years (range, 26-72) \\
\hline Performance Status & & $0(0-2)$ \\
Races/Ethnicities & Non-Hispanic White & 8 \\
& Hispanic White & 7 \\
Histology & Black & 2 \\
& & Male/Female \\
& Undifferentiated sarcoma & $4 / 1$ \\
& Leiomyosarcoma & $0 / 4$ \\
& Liposarcoma & $1 / 2$ \\
Prior Therapies & Myxofibrosarcoma & $3 / 0$ \\
& Peripheral Nerve Sheath Tumor & $1 / 0$ \\
& Extraosseous osteosarcoma & $1 / 0$ \\
& Surgery & 14 \\
& Radiotherapy & 8 \\
& One prior chemotherapy & 13 \\
& More than one chemotherapy & 4 \\
\hline
\end{tabular}

Three patients were enrolled on Arm B (fixed dose of temsirolimus $25 \mathrm{mg}$ and a starting dose of irinotecan of $50 \mathrm{mg} / \mathrm{m}^{2}$ ). All three patients had a dose limiting toxicity (DLT); grade 3 neutropenia on retreatment day 8 in one patient, and inability to receive three doses of weekly treatment in two patients who described gastrointestinal symptoms of nausea, emesis, and diarrhea. One of these two patients received the first dose and refused further administrations. He also had a DLT of grade 3 muscle weakness, related to electrolyte imbalance from the gastrointestinal symptoms. The other patient had a second administration at a reduced dose of temsirolimus from $25 \mathrm{mg}$ to $20 \mathrm{mg}$, and then refused the third dose. Thus this arm was closed. Arm A (fixed dose of irinotecan at $80 \mathrm{mg} / \mathrm{m}^{2}$ and a starting dose of temsirolimus of $15 \mathrm{mg}$ ) was completed after fourteen patients were accumulated, six at the starting level and eight at the next dose level (fixed dose of irinotecan at $80 \mathrm{mg} / \mathrm{m}^{2}$ and temsirolimus at $20 \mathrm{mg}$ ). There was one patient with a DLT at the starting dose who did not recover the platelet count to $100,000 / \mathrm{mm}^{3}$ at the end of the 4 th week. Thus, three additional patients were treated at that level and no further DLT were observed. The second cohort was the last cohort since $25 \mathrm{mg}$ per week of temsirolimus could not be combined with a lower dose of irinotecan in Arm B. A total of eight patients were enrolled at that level. No DLTs were observed. Because no objective responses were seen in these patients, this regimen was not sufficiently active in refractory soft tissue sarcoma and part 2 of the study was never initiated. Adverse events are described in Table 2. The hematological side effects were manageable, usually a grade 1 or 2 in a third of the patients, but timely recovery of blood counts did not always happen with additional cycles of therapy, indicating a possible cumulative toxicity. Other side effects included gastrointestinal intolerance with nausea, diarrhea and vomiting, again in a third of the patients, as well as fatigue and skin rash. 


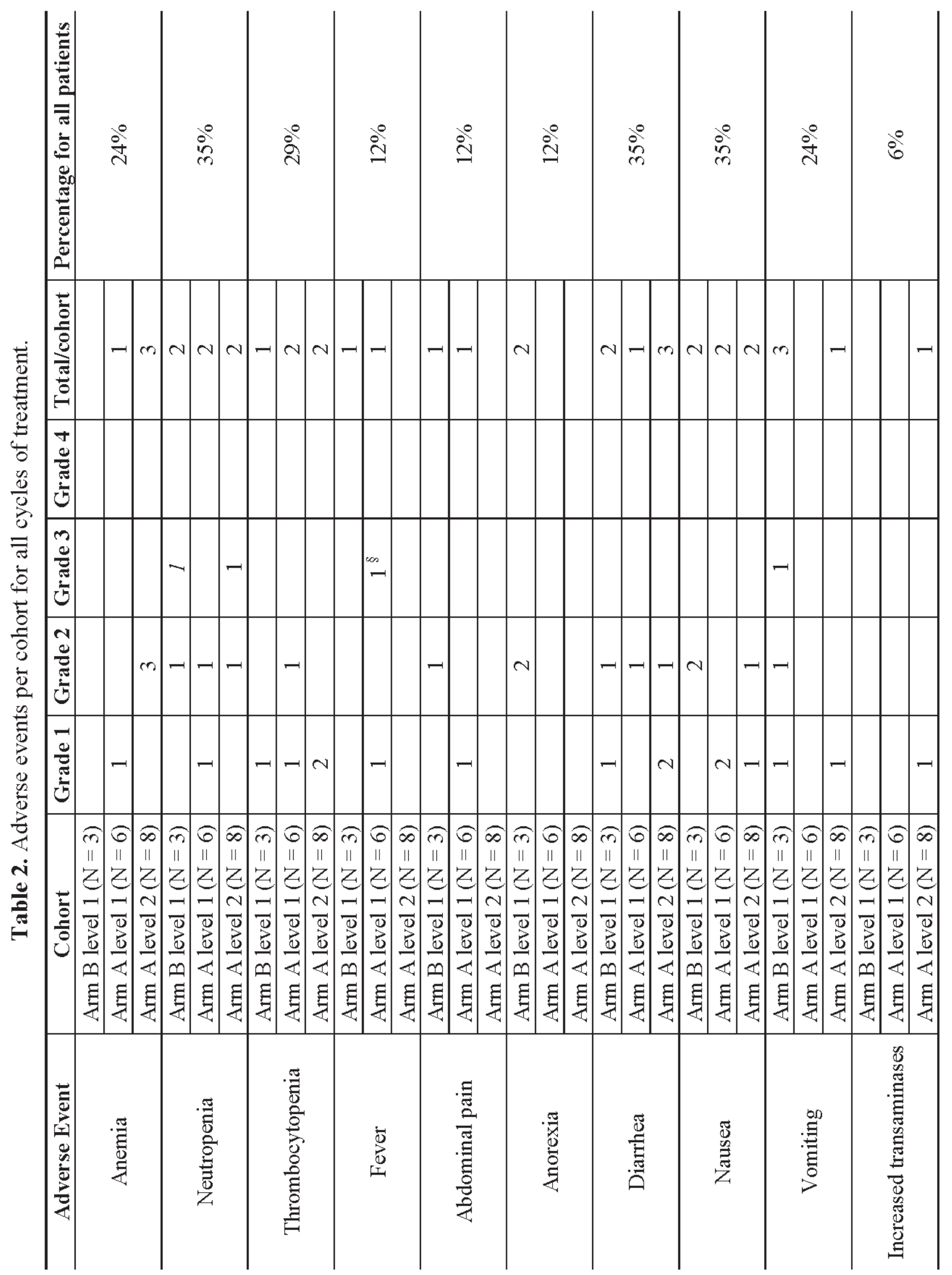




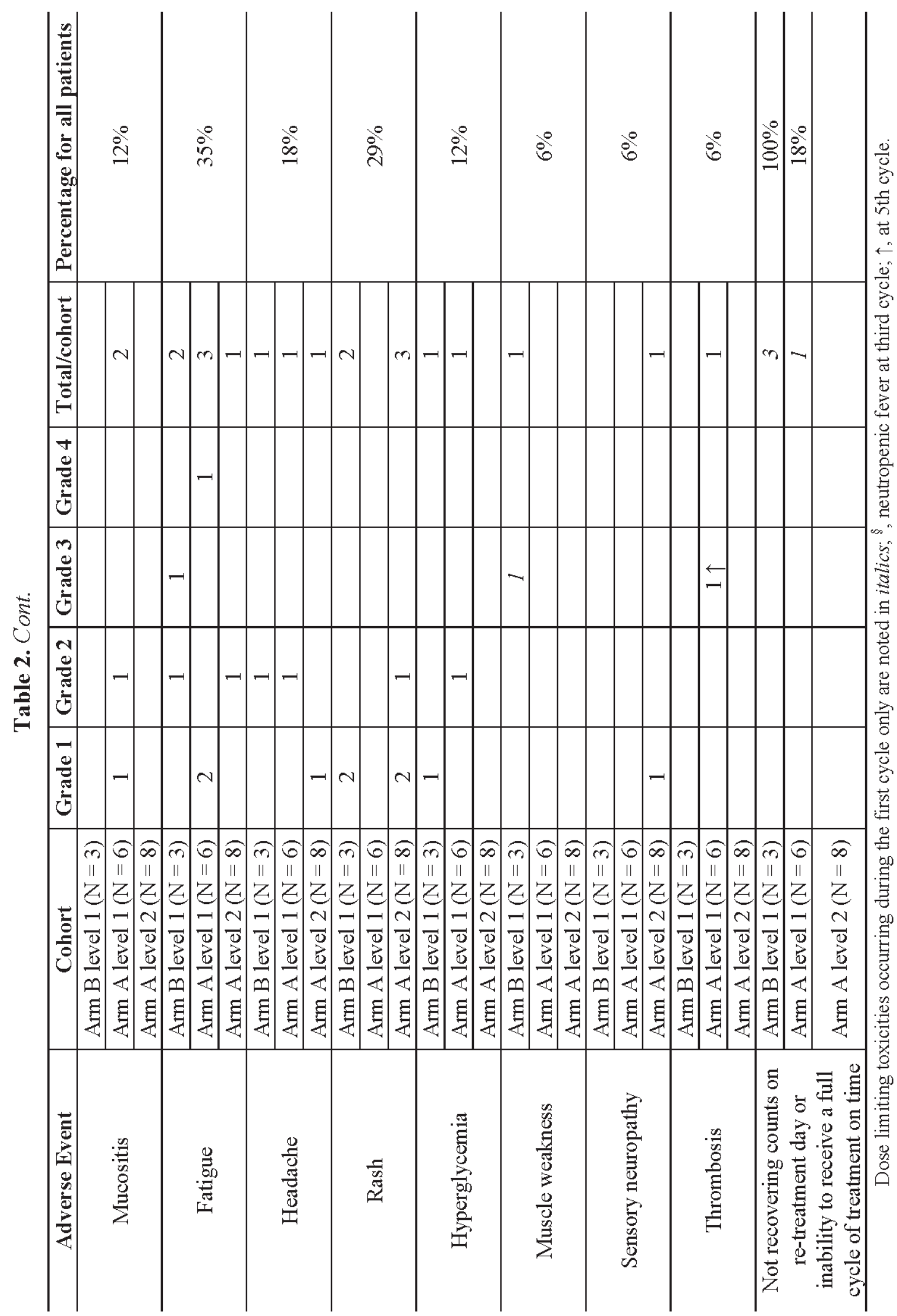

There were no objective responses. However, two patients who had progressive disease prior to entry into the study stabilized their disease for 12 cycles each. One had a leiomyosarcoma, and the other was diagnosed with an undifferentiated grade 3 sarcoma and is the only patient who remains 
alive two years later. Two more patients, one with a liposarcoma and one with an undifferentiated sarcoma, received four cycles each. All other patients progressed after two cycles.

\section{Conclusions}

Disruption of PI3K-AKT-mTOR pathways has been associated with different sarcoma subtypes. Rapamycin and its analogs, which bind to the intracellular 12-kDa FK506-binding protein (FKBP-12) to form a protein-drug complex, inhibit the activity of mTOR [27-30]. The growth of multiple sarcoma cell lines, including rhabdomyosarcoma [31], osteosarcoma cell lines [32], and Ewing sarcoma [33], is inhibited by the rapamycin analogs. A multicenter phase II study of rapamycin in patients with soft tissue sarcoma did not appear to be effective when administrated as a monotherapy, although one patient with fibrosarcoma achieved a partial response after two cycles lasting at least 36 weeks [34]. Subsequently, ridaforolimus showed promising efficacy in various trials enrolling patients with advanced sarcoma [15,35], but as an indication for the maintenance of remission in patients treated for metastatic sarcoma it was not approved because of the failure of the drug to exhibit a meaningful clinical activity [36]. In combination, the mTOR inhibitor, sirolimus, with cyclophosphamide is tolerated by the majority of sarcoma patients with a clinical benefit rate of about $20 \%$ [37].

In this study, we attempted to capitalize on in vitro data suggesting that mTOR inhibition could enhance tumor cell killing by DNA damaging agents such as irinotecan through the HIF-1 $\alpha$ pathway. The aim of this study was to identify the MTD of the combination of irinotecan and temsirolimus in patients with refractory sarcomas, followed by expansion of this patients' cohort to probe clinical activity. We did identify a do se of irinotecan of $80 \mathrm{mg} / \mathrm{m}^{2}$ and temsirolimus of $20 \mathrm{mg}$ weekly for three consecutive weeks every four weeks as the MTD. However, after enrolling a total of 17 patients, we did not appreciate significant clinical activity (only two patients with stable disease) to warrant further expansion in this patient population. We have proved that the two drugs can be safely administered clinically at the aforementioned doses, and could be studied in other tumor types in the future. Temsirolimus appears to enhance the toxicities usually seen with irinotecan in terms of gastrointestinal and hematological adverse events. When the full dose of temsirolimus was given with low-dose weekly irinotecan, the regimen was not tolerated. The combination of irinotecan and temsirolimus did not meet the expectations of this study. However, in two patients this regimen stabilized the disease for about one year. This amounts to a $12 \%$ clinical benefit rate, which is similar to that published for mTOR inhibitors as single agent. One patient had a leiomyosarcoma, where alterations of the mTOR pathway have been described, such as the overexpression of RICTOR, a major component of the mTOR complex 2, which might contribute to the pathogenesis of well-differentiated leiomyosarcoma [38,39]. Unfortunately, there is no proven clinical biomarker to predict the activity of mTOR inhibitors against sarcomas. Thus further understanding of the role of the mTOR pathway in the oncogenesis of sarcomas is warranted. Prolonged stabilization of disease with a low toxicity regimen could be useful if the susceptible patients could be appropriately selected. 


\section{Acknowledgments}

Support for the study was obtained from Wyeth (temsirolimus and support for data management). We thank Chantal Garrett, Terry Novak, and Mary Pruess for data management and research nursing support.

\section{References}

1. Laplante, M.; Sabatini, D.M. mTOR signaling in growth control and disease. Cell 2012, 149, 274-293.

2. Wong, K.K.; Engelman, J.A.; Cantley, L.C. Targeting the PI3K signaling pathway in cancer. Curr. Opin. Genet. Dev. 2010, 20, 87-90.

3. Cully, M.; Downward, J. Translational responses to growth factors and stress. Biochem. Soc. Trans. 2009, 37, 284-288.

4. Sleijfer, S.; van der Graaf, W.T.; Blay, J.Y. Angiogenesis inhibition in non-GIST soft tissue sarcomas. Oncologist 2008, 13, 1193-1200.

5. Falcon, B.L.; Barr, S.; Gokhale, P.C.; Chou, J.; Fogarty, J.; Depeille, P.; Miglarese, M.; Epstein, D.M.; McDonald, D.M. Reduced VE GF production, angiogenesis, and vascular regrowth contribute to the antitumor properties of dual mTORC1/mTORC2 inhibitors. Cancer Res. 2011, 71, 1573-1583.

6. Levine, A.J.; Feng, Z.; Mak, T.W.; You, H.; Jin, S. Coordination and communication between the p53 and IGF-1-AKT-TOR signal transduction pathways. Genes Dev. 2006, 20, 267-275.

7. Hudes, G.; Carducci, M.; Tomczak, P.; Dutcher, J.; Figlin, R.; Kapoor, A.; Staroslawska, E.; Sosman, J.; McDermott, D.; Bodrogi, I.; et al. Temsirolimus, interferon alfa, or both for advanced renal-cell carcinoma. N. Engl. J. Med. 2007, 356, 2271-2281.

8. Motzer, R.J.; Hudes, G.R.; Curti, B.D.; McDermott, D.F.; Escudier, B.J.; Negrier, S.; Duclos, B.; Moore, L.; O'Toole, T.; Boni, J.P.; et al. Phase I/II trial of temsirolimus combined with interferon alfa for advanced renal cell carcinoma. J. Clin. Oncol. 2007, 25, 3958-3964.

9. Motzer, R.J.; Escudier, B.; Oudard, S.; Hutson, T.E.; Porta, C.; Bracarda, S.; Grunwald, V.; Thompson, J.A.; Figlin, R.A.; Hollaender, N.; et al. Efficacy of everolimus in advanced renal cell carcinoma: A double-blind, randomised, placebo-controlled phase III trial. Lancet 2008, 372, 449-456.

10. Pavel, M.E.; Hainsworth, J.D.; Baudin, E.; Peeters, M.; Horsch, D.; Winkler, R.E.; Klimovsky, J.; Lebwohl, D.; Jehl, V.; Wolin, E.M.; et al. Everolimus plus octreotide long-acting repeatable for the treatment of advanced neuroendocrine tumours associated with carcinoid syndrome (RADIANT-2): A randomised, placebo-controlled, phase 3 study. Lancet 2011, 378, 2005-2012.

11. Baselga, J.; Campone, M.; Piccart, M.; Burris, H.A., 3rd; Rugo, H.S.; Sahmoud, T.; Noguchi, S.; Gnant, M.; Pritchard, K.I.; Lebrun, F.; et al. Everolimus in postmenopausal hormone-receptor-positive advanced breast cancer. N. Engl. J. Med. 2012, 366, 520-529.

12. Dickson, M.A.; Schwartz, G.K.; Antonescu, C.R.; Kwiatkowski, D.J.; Malinowska, I.A. Extrarenal perivascular epithelioid cell tumors (PEComas) respond to mTOR inhibition: Clinical and molecular correlates. Int. J. Cancer 2013, 132, 1711-1717. 
13. Dabora, S.L.; Franz, D.N.; Ashwal, S.; Sagalowsky, A.; DiMario, F.J., Jr.; Miles, D.; Cutler, D.; Krueger, D.; Uppot, R.N.; Rabenou, R.; et al. Multicenter phase 2 trial of sirolimus for tuberous sclerosis: Kidney angiomyolipomas and other tumors regress and VEGF-D levels decrease. PLoS One 2011, 6, e23379.

14. Okuno, S.; Bailey, H.; Mahoney, M.R.; Adkins, D.; Maples, W.; Fitch, T.; Ettinger, D.; Erlichman, C.; Sarkaria, J.N. A phase 2 study of temsirolimus (CCI-779) in patients with soft tissue sarcomas: A study of the Mayo phase 2 c onsortium (P2C). Cancer 2011, 117, $3468-3475$.

15. Chawla, S.P.; Staddon, A.P.; Baker, L.H.; Schuetze, S.M.; Tolcher, A.W.; D’Amato, G.Z.; Blay, J.Y.; Mita, M.M.; Sankhala, K.K.; Berk, L.; et al. Phase II study of the mammalian target of rapamycin inhibitor ridaforolimus in patients with advanced bone and soft tissue sarcomas. J. Clin. Oncol. 2012, 30, 78-84.

16. Chawla, S.; Blay, J.; Ray-Coquard, L.; Cesne, A.; Staddon, A.; Milhem, M.; Penel, N.; Riedel, R.; Nguyen, B.; Cranmer, L.; et al. Results of the phase III, placebo-controlled trial (SUCCEED) evaluating the mTOR inhibitor ridaforolimus (R) as maintenance therapy in advanced sarcoma patients (pts) following clinical benefit from prior standard cytotoxic chemotherapy. J. Clin. Oncol. 2011, 29, A10005.

17. Creel, P.A. Management of mTOR inhibitor side effects. Clin. J. Oncol. Nurs. 2009, 13, $19-23$.

18. Alagoz, M.; Gilbert, D.C.; El-Khamisy, S.; Chalmers, A.J. DNA repair and resistance to topoisomerase I inhibitors: Mechanisms, biomarkers and therapeutic targets. Curr. Med. Chem. 2012, 19, 3874-3885.

19. Jackson, S.P. The DNA-damage response: New molecular insights and new appr oaches to cancer therapy. Biochem. Soc. Trans. 2009, 37, 483-494.

20. Casey, D.A.; Wexler, L.H.; Merchant, M.S.; Chou, A.J.; Merola, P.R.; Price, A.P.; Meyers, P.A. Irinotecan and temozolomide for Ewing sarcoma: The Memorial Sloan-Kettering experience. Pediatr. Blood Cancer 2009, 53, 1029-1034.

21. Pappo, A.S.; Lyden, E.; Breitfeld, P.; Donaldson, S.S.; Wiener, E.; Parham, D.; Crews, K.R.; Houghton, P.; Meyer, W.H. Two consecutive phase II window trials of irinotecan alone or in combination with vincristine for the treatment of metastatic rhabdomyosarcoma: The Children's Oncology Group. J. Clin. Oncol. 2007, 25, 362-369.

22. Vassal, G.; Couanet, D.; Stockdale, E.; Geoffray, A.; Geoerger, B.; Orbach, D.; Pichon, F.; Gentet, J.C.; Picton, S.; Bergeron, C.; et al. Phase II trial of irinotecan in children with relapsed or refractory rhabdomyosarcoma: A joint study of the French Society of Pediatric Oncology and the United Kingdom Children's Cancer Study Group. J. Clin. Oncol. 2007, 25, 356-361.

23. Bisogno, G.; Riccardi, R.; Ruggiero, A.; Arcamone, G.; Prete, A.; Surico, G.; Provenzi, M.; Bertolini, P.; Paolucci, P.; Carli, M. Phase II study of a protracted irinotecan schedule in children with refractory or recurrent soft tissue sarcoma. Cancer 2006, 106, 703-707.

24. Eckhardt, S.G. Irinotecan: A review of the initial phase I trials. Oncology 1998, 12, 31-38. 
25. Pencreach, E.; Guerin, E.; Nicolet, C.; Lelong-Rebel, I.; Voegeli, A.C.; Oudet, P.; Larsen, A.K.; Gaub, M.P.; Guenot, D. Marked activity of irinotecan and rapamycin combination toward colon cancer cells in vivo and in vitro is mediated through cooperative modulation of the mammalian target of rapamycin/hypoxia-inducible factor-1alpha axis. Clin. Cancer Res. 2009, 15, 1297-1307.

26. Lee, S.J.; Gounder, M.; Rubin, E.H.; Li, J.M.; Gu, Z.; Thalasila, A.; Loyer, E.; Kudelka, A.P.; Verschraegen, C.F. Optimal modeling for phase I design of a two drug combination-results of a phase I study of cisplatin with 9-nitrocamptothecin. Investig. New Drugs 2008, 26, 541-551.

27. Back, J.H.; Rezvani, H.R.; Zhu, Y.; Guyonnet-Duperat, V.; Athar, M.; Ratner, D.; Kim, A.L. Cancer cell survival following DNA damage-mediated premature senescence is regulated by mammalian target of rapamycin (mTOR)-dependent Inhibition of sirtuin 1. J. Biol. Chem. 2011, 286, 19100-19108.

28. Sabatini, D.M.; Erdjument-Bromage, H.; Lui, M.; Tempst, P.; Snyder, S.H. RAFT1: A mammalian protein that binds to FKBP12 in a r apamycin-dependent fashion and is homologous to yeast TORs. Cell 1994, 78, 35-43.

29. Boni, J.P.; Hug, B.; Leister, C.; Sonnichsen, D. Intravenous temsirolimus in cancer patients: Clinical pharmacology and dosing considerations. Semin. Oncol. 2009, 36, S18-S25.

30. Klumpen, H.J.; Beijnen, J.H.; Gurney, H.; Schellens, J.H. Inhibitors of mTOR. Oncologist 2010, 15, 1262-1269.

31. Dilling, M.B.; Dias, P.; Shapiro, D.N.; Germain, G.S.; Johnson, R.K.; Houghton, P.J. Rapamycin selectively inhibits the growth of childhood rhabdomyosarcoma cells through inhibition of signaling via the type I insulin-like growth factor receptor. Cancer Res. 1994, 54, 903-907.

32. Ogawa, T.; Tokuda, M.; Tomizawa, K.; Matsui, H.; Itano, T.; Konishi, R.; Nagahata, S.; Hatase, O. Osteoblastic differentiation is enhanced by rapamycin in rat osteoblast-like osteosarcoma (ROS 17/2.8) cells. Biochem. Biophys. Res. Commun. 1998, 249, 226-230.

33. Mateo-Lozano, S.; Tirado, O.M.; Notario, V. Rapamycin induces the fusion-type independent downregulation of the EWS/FLI-1 proteins and inhibits Ewing's sarcoma cell proliferation. Oncogene 2003, 22, 9282-9287.

34. Schuetze, S.; Baker, L.; Maki, R. Sirolimus reduced tumor-related morbidity and resulted in biochemical and radiographic response in patients with progressive sarcoma. J. Clin. Oncol. 2006, 24, A9503.

35. Mita, M.; Sankhala, K.; Abdel-Karim, I.; Mita, A.; Giles, F. Deforolimus (AP23573) a novel mTOR inhibitor in clinical development. Expert Opin. Investig. Drugs 2008, 17, 1947-1954.

36. Garrido-Laguna, I.; Janku, F. Ridaforolimus in advanced sarcomas: A leap forward or missed opportunity? J. Clin. Oncol. 2012, 30, 892-893.

37. Schuetze, S.M.; Zhao, L.; Chugh, R.; Thomas, D.G.; Lucas, D.R.; Metko, G.; Zalupski, M.M.; Baker, L.H. Results of a phase II study of sirolimus and cyclophosphamide in patients with advanced sarcoma. Eur. J. Cancer 2012, 48, 1347-1353. 
38. Gibault, L.; Ferreira, C.; Perot, G.; Audebourg, A.; Chibon, F.; Bonnin, S.; Lagarde, P.; Vacher-Lavenu, M.C.; Terrier, P.; Coindre, J.M.; et al. From PTEN loss of expression to RICTOR role in smooth muscle differentiation: Complex involvement of the mTOR pathway in leiomyosarcomas and pleomorphic sarcomas. Mod. Pathol. 2012, 25, 197-211.

39. Italiano, A.; Kind, M.; Stoeckle, E.; Jones, N.; Coindre, J.M.; Bui, B. Temsirolimus in advanced leiomyosarcomas: Patterns of response and correlation with the activation of the mammalian target of rapamycin pathway. Anticancer Drugs 2011, 22, 463-467. 
Reprinted from Cancers. Cite as: L adra, M.M.; Yock, T.I. Proton Radiotherapy for Pediatric Sarcoma. Cancers 2014, 6, 112-127.

Review

\title{
Proton Radiotherapy for Pediatric Sarcoma
}

\section{Matthew M. Ladra and Torunn I. Yock*}

Department of Radiation Oncology, Massachusetts General Hospital, Boston, MA 02114, USA; E-Mail:mladra@partners.org

* Author to whom correspondence should be addressed; E-Mail: tyock@partners.org; Tel.: +1-617-726-6876; Fax: +1-617-724-4808.

Received: 20 October 2013; in revised form: 17 December 2013 / Accepted: 18 December 2013 / Published: 14 January 2014

\begin{abstract}
Pediatric sarcomas represent a di stinct group of pathologies, with approximately 900 new cases per year in the United States alone. Radiotherapy plays an integral role in the local control of these tumors, which often arise adjacent to critical structures and growing organs. The physical properties of proton beam radiotherapy provide a distinct advantage over standard photon radiation by eliminating excess dose deposited beyond the target volume, thereby reducing both the dose of radiation delivered to non-target structures as well as the total radiation dose delivered to a patient. Dosimetric studies comparing proton plans to IMRT and 3D conformal radiation have demonstrated the superiority of protons in numerous pediatric malignancies and data on long-term clinical outcomes and toxicity is emerging. In this article, we review the existing clinical and dosimetric data regarding the use of proton beam radiation in malignant bone and soft tissue sarcomas.
\end{abstract}

Keywords: sarcoma; pediatrics; protons; ewings; osteosarcoma; rhabdomyosarcoma; chordoma; chondrosarcoma

\section{Introduction}

Pediatric sarcomas represent a diverse and challenging group of malignancies. Treatment requires a varied, multidisciplinary approach based on histology and location. Adjuvant radiation remains a mainstay of local therapy but is associated with significant acute and long-term treatment morbidity within multiple organ systems and an increased risk of second malignancy [1]. Advances in surgical technique, systemic therapy, and radiotherapy imaging and delivery have increased survival across all sarcoma subtypes. With improved clinical outcomes, greater emphasis becomes focused on 
reducing long-term toxicity in these young patients. Proton therapy has become a useful tool in the drive to limit acute and late effects.

Proton therapy as large field fractionated treatment was first used in clinical practice in the United States at the Harvard Cyclotron Laboratory in Cambridge, MA beginning in 1974. To date more than 74,000 patients have been treated with proton radiotherapy worldwide. Internationally there are 36 active proton facilities, including 11 within the United States, and additional centers are under development. Currently, the capital cost of constructing a multi-gantry proton center exceeds 100 million dollars. Newer treatment designs in production aim to decrease this cost, and smaller single-gantry facilities are being developed and offered in the range of 20-30 million dollars. At present, the cost ratio of delivering a fraction of proton radiation is 2.4 times that of photon radiation [2], although with decreasing technical costs this ratio is expected to drop significantly.

Because the relative biologic effectiveness (RBE) of protons is nearly equivalent to that of high-energy X-rays $(\mathrm{RBE}=1.1)$, the interest in protons is based primarily on physical properties that provide favorable dose distributions [3]. Compared to photons, protons deposit a nearly constant quantity of radiation until the end of the beam range, where the majority of energy is released over a short distance called the Bragg Peak, roughly 5-10 mm in length. To cover a target of larger size, beam modulation is used to create a "spread out" Bragg Peak (SOBP) that expands the area of high-energy deposition to cover the desired target length. As opposed to photon beams which have a gradual dose fall off after reaching the target depth in a patient, the Bragg Peak in proton beams leads to a complete absence of "exit dose", or dose beyond the target volume. A comparison of depth dose curves for a $10 \mathrm{MV}$ photon beam, a $10 \mathrm{MeV}$ proton beam, and SOBP are shown in Figure 1. Through the elimination of exit dose, protons can provide significant advantages over photons by decreasing the dose to adjacent critical structures such as the spinal cord, brainstem, or kidneys, and allowing for increased total dose to be given to the target volume. Further, the "integral dose" or total energy deposited in a patient from radiation therapy is decreased significantly. In dosimetric comparisons, the integral dose per patient treated with protons is decreased by a factor of 2-3 compared to photon therapy [4]. Typically, proton dose is prescribed in cobalt Gray equivalent (CGE) units, using an RBE of 1.1. For example, a proton dose of 50.4 CGE represents an energy deposit equivalent to 45.8 Gy of photon radiation but has an in vivo effect of $50.4 \mathrm{~Gy}$, attributable to the increased RBE. For clarity and ease of presentation, the proton doses discussed in this review will be written using "Gy", but it should be noted that the units represent the CGE.

The potential for reduced secondary malignancy attributable to improved dose distribution with proton beam radiation was examined in a publication by Miralbell et al. [5]. Three treatment plans (3D conformal photon, IMRT, and proton) were generated and compared for one patient with medulloblastoma, and four plans (3D, IMRT, proton, and intensity modulated proton therapy (IMPT)), were created for a rhabdomyosarcoma (RMS) of the paranasal sinus. The absolute risks of secondary cancer for each treatment plan was estimated based on dose-volume distributions for the non-target organs using models from the International Commission on Radiologic Protection. Proton beam reduced the expected incidence of radiation-induced secondary cancers for the RMS patient by a factor of more than 2, and for the medulloblastoma patient by a factor of 8 to 15 when compared with either IMRT or 3D conformal radiation. Taken together, these properties provide a strong impetus for the use of proton therapy in children. 
Figure 1. Comparison of depth dose curves for a $10 \mathrm{MV}$ photon beam and a $10 \mathrm{MeV}$ proton beam (shown with and without a SOBP). This figure shows the decreased entrance dose and absence of exit dose for the proton beam in comparison to the photon beam.

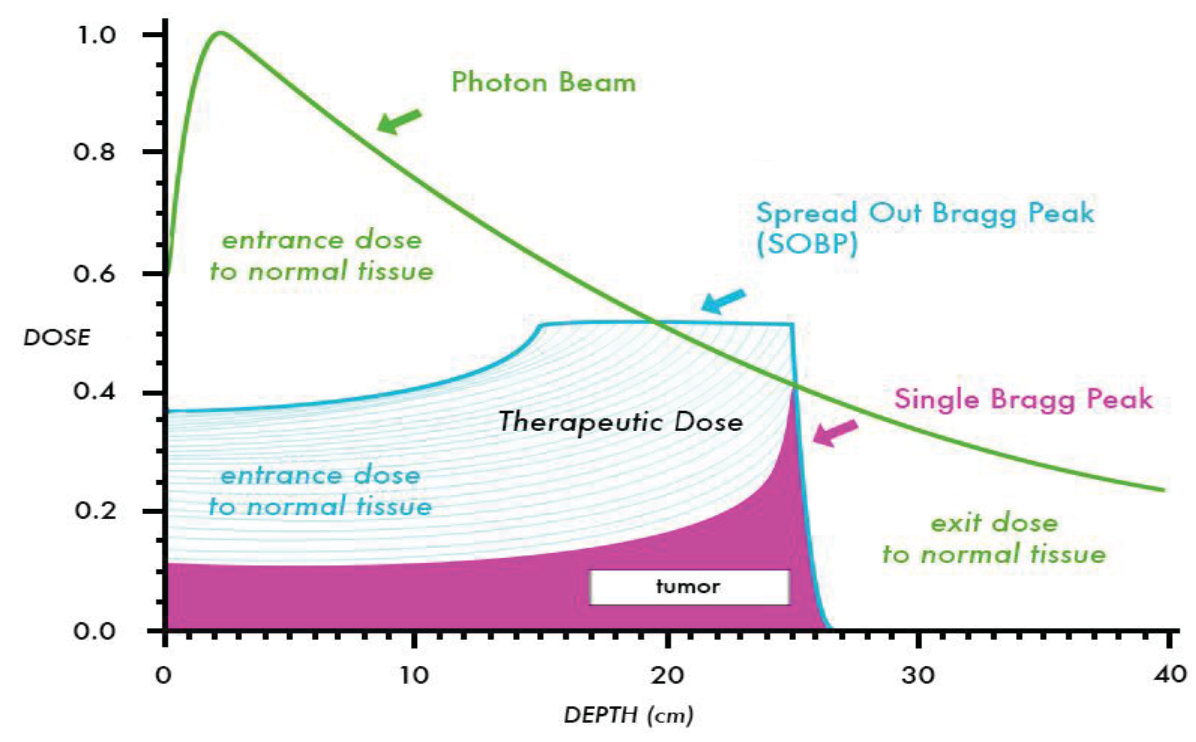

\section{Rhabdomyosarcoma and Soft Tissue Sarcoma}

Rhabdomyosarcoma (RMS) represents the most common pediatric soft tissue sarcoma, with approximately 350 new cases per year within the United States [6]. There is a bimodal age distribution, with two thirds of the cases occurring before the age of ten, followed by a second peak in adolescence. These tumors arise in a variety of locations, including the head and neck (35\%), genitourinary tract $(24 \%)$, limbs $(19 \%)$, and elsewhere $(22 \%)$, and only one-third are readily resectable [7]. RMS patients are stratified into low, intermediate and high-risk groups based on site of origin, size, histology, lymph node involvement, metastatic spread, and extent of surgical resection [8]. Patients with low risk disease who undergo a complete resection do not need radiation. Low risk patients with a gross total resection and positive margins receive $36 \mathrm{~Gy}$, those with positive nodes $41.4 \mathrm{~Gy}$ and those with gross residual disease $50.4 \mathrm{~Gy}$. Intermediate risk patients, those with alveolar histology or those with gross disease at the start of chemotherapy, will receive radiation therapy (RT) regardless of surgical margin status if delayed resection is done. Children with orbital primaries are treated to 45 Gy after biopsy alone. All patients typically receive VAC- or VAI-(vincristine, dactinomycin, and cyclophosphamide or ifosfamide) based chemotherapy.

As many of these tumors arise in close proximity to critical structures in developing children, the incorporation of proton RT into treatment has been explored and the recently closed Children's Oncology Group (COG) and International Rhabdomyosarcoma Study Group (IRSG) protocols allowed for the use of proton RT to treat patients. At Massachusetts General Hospital (MGH), proton RT has been used to treat pediatric RMS patients with protons since the 1990s. The 3-year outcomes for 57 RMS patients enrolled on a prospective joint proton protocol with MD Anderson Cancer Center (MDACC) between 2005 and 2012 were recently presented in abstract form [9]. Median three-year local control (LC), progression free survival (PFS) and overall survival (OS) were $82 \%$, 
$75 \%$, and $81 \%$ respectively, comparable to published results from the IRS IV/V trials [10-12]. The protocol included those with localized disease and patients with metastatic embryonal RMS between the ages of 2-10 years. No patients developed acute or late toxicity of grade 4 or 5 and there were three incidences of grade 3 late toxicity, related to chronic otitis, cataract, and dry eye in head and neck tumors.

Several smaller site-specific studies using protons in RMS have been published. A retrospective review by Childs et al. of 17 consecutive children with parameningeal rhabdomyosarcoma (PM-RMS) that were treated with proton radiotherapy between 1996 an d 2005, demonstrated comparable outcomes to that seen with standard photon RT [13]. From the photon literature, local control for children with PM-RMS ranges from $80 \%-90 \%$, and the 5-year failure free survival (FFS) is approximately $67 \%$, but can drop as low as $52 \%$ if a patient has an unfavorable parameningeal site and meningeal impingement $[1,8,12]$. In the proton study, the median age for this study was 3.4 years and mean dose was 50.4 Gy (range, 50.4-56.0 Gy). The 5-year failure-free survival was 59\%, and overall survival was $64 \%$. Patients who had intracranial extension at diagnosis $(n=10)$ had a 5 -year FFS of $50 \%$ and a 5 -year OS of $60 \%$. Patients without intracranial extension $(n=7)$ fared better with a 5 -year FFS of $71 \%$ and a 5 -year OS of $71 \%$. Seven of the 17 patients $(41 \%)$ had a recurrence at a median time of 10.5 months (range, 7-18.5 months) after diagnosis. Among the seven patients who failed, sites of first recurrence were local only $(n=2)$, regional only $(n=2)$, distant only $(n=2)$, and a single patient failed both locally and distant. Ten patients $(59 \%)$ were without tumor recurrence at study completion and available for late toxicity evaluation. Among these patients, late effects of multimodality treatment included mild facial hypoplasia $(n=7)$, lack of permanent tooth eruption $(n=3)$, decreased height velocity $(n=3)$, endocrinopathies $(n=2)$ and chronic sinus congestion $(n=2)$. These numbers compare favorably to long term studies of traditional photon based treatment where late toxicity for head and neck patients included hearing loss $(17 \%-20 \%)$, learning disabilities $(10 \%-49 \%)$, facial/orbital hypoplasia $(34 \%-36 \%)$ visual problems $(17 \%-30 \%)$, decreased height velocity $(30 \%-61 \%)$ and poor dentition $(23 \%-29 \%)[14,15]$.

A separate dosimetric comparison of proton and intensity-modulated photon radiotherapy for 10 pediatric PM-RMS patients was also published by the same group [16]. Each patient was treated with protons, and the proton treatment plan was then compared to an IMRT plan generated for study purposes. In nine cases, the prescribed GTV dose was 50.4 Gy in 28 fractions. In the remaining case, the prescribed GTV dose was 52.2 Gy in 29 fractions. The median CTV prescription dose was 47.7 Gy (range, 36-52.2 Gy). Comparative beam arrangements were given for each proton and photon plan and on average five beams were used in the proton plans and four were used in the photon plans.

Eight patients in this cohort had radiographic evidence of intracranial extension. Proton treatment yielded equivalent target coverage while significantly decreasing dose to the optic structures (globe, lens, optic nerves, optic chiasm, and retina), the hypothalamic-pituitary axis, the brainstem, temporal lobes, cochlea, lacrimal glands, and parotids. Although direct comparison of clinical outcomes could not be done, some differences between the proton and photon plans were quite striking. With regards to lens dose and cataract risk, only one proton plan had a lens dose higher than $5 \mathrm{~Gy}$, whereas in the photon plans $80 \%$ of ipsilateral lenses and $60 \%$ of contralateral lenses received above 5 Gy. Cochlear dose associated with hearing loss is minimized at doses less than $32 \mathrm{~Gy}$, and no proton plans had a mean contralateral dose above 27 Gy whereas $50 \%$ of photon plans had a contralateral cochlear dose 
over 32 Gy. Similarly, for contralateral parotid glands where long-term salivary dysfunction begins at 26-30 Gy, the mean proton dose never exceeded 13 Gy whereas the mean dose for the IMRT plans was greater than 26 Gy for $70 \%$ of cases. And finally, the mean dose to the hypothalamus was 12 Gy for protons vs. $22.4 \mathrm{~Gy}$ for IMRT. Merchant et al. recently published data showing that a cumulative dose of 16.1 Gy to the hypothalamus was the mean radiation dose required to achieve a $50 \%$ risk of growth hormone deficiency at 5 years [17].

The proton experience for orbital RMS was reported by Yock et al. [18]. Seven children were treated to a median dose of 46.6 Gy with a median follow-up of 6.3 years. Six of the seven patients were without evidence of disease and the remaining child was salvaged with exenteration and stereotactic radiosurgery after local recurrence. The child with local failure had a two unfavorable prognostic factors going into RT; progression during chemotherapy and an age less than 1 year. Late effects of treatment were minimal. All six patients retained good vision in the treated eye and two of the six required drops for lubrication but none demonstrated corneal pathology or dry eye syndrome. All patients did develop mild to moderate orbital bony asymmetry or enophthalmous. None of the patients developed neuroendocrine deficits. In the early IRS trials (I-III), diminished height velocity, a marker for hypothalamic-pituitary dysfunction, was $48 \%-61 \%$ for orbital tumors and in more recent IMRT trials including orbital tumors, neuroendocrine dysfunction has ranged from $3 \%-10 \%$ for the small number of patients analyzed $[14,15,19,20]$. In the publication, the authors also compared the proton plans used for treatment to 3D conformal plans generated for the study. Proton radiation markedly decreased dose to the brain, temporal lobes, hypothalamic-pituitary axis, and both the ipsilateral and contralateral orbital structures with an average percent savings of $82 \%-94 \%$ for CNS structures and 26\%-65\% for ipsilateral orbital structures.

Finally, Cotter et al. reported the outcomes of for seven children treated with protons for bladder/prostate RMS with a median follow-up of 27 months [21]. Patients had a mean age of 30 months (range: 11-70) and radiation dose ranged from to 36 to 50.4 Gy. Five of seven patients (71.4\%) were without evidence of disease with intact bladders at study completion. One patient had a local recurrence in the treatment field, while a second had a local and a distant recurrence. Two of the five patients with intact bladders at the end of treatment reported bladder dysfunction, both of which were attributable to prior surgical procedures. No long-term skeletal or gastrointestinal effects were noted, and all patients were too young to assess sexual function. IMRT plans were created for study purposes and compared to the proton plans used for treatment. Proton radiotherapy showed a statistically significant decrease in mean organ dose to the bladder (median proton dose of 25 Gy vs. median IMRT dose of $33.2 \mathrm{~Gy} ; p=0.03$ ), testes (0.0 CGE $v s .0 .6 \mathrm{~Gy} ; p=0.016$ ), femoral heads (1.6 Gy vs. $10.6 \mathrm{~Gy} ; p=0.016$ ), pelvic growth plates (21.7 Gy vs. $32.4 \mathrm{~Gy} ; p=0.016$ ), and pelvic bones ( $8.8 \mathrm{~Gy}$ vs. $13.5 \mathrm{~Gy} ; p=0.016)$. There were no significant differences seen in dose to the bowel, prostate, penile bulb, and rectum.

Pelvic dose sparing in sarcoma treated with protons was also demonstrated in a do simetric analysis from MD Anderson Cancer Center [22]. A total of three patients with pelvic sarcomas, including one RMS, were planned using various techniques and demonstrated a marked reduction in ovarian dose with protons compared to both 3D conformal and IMRT. Protons allowed for complete sparing of dose to the ovaries while IMRT led to $100 \%$ of the ovarian volume receiving 2 Gy and $30 \%$ receiving a mean dose of $5 \mathrm{~Gy}$. Significant dose sparing to the pelvic bones and vertebral bodies 
was also demonstrated with protons, and the mean volume receiving $20 \mathrm{~Gy}$ was $13 \%$ and $29 \%$ with IMRT vs. 5\% and 9\% with protons. IMRT was associated with slightly better bladder sparing in these cases with $2 \%$ vs. $8 \%$ receiving $40 \mathrm{G}$ y and $0 \%$ vs. $4 \%$ receiving $45 \mathrm{G}$ y. Some examples of comparative proton and photon plans are shown in Figure 2.

Figure 2. Isodose comparisons for photon and proton plans. (A) Typical parameningeal (left) and orbital (right) plans for pediatric RMS. IMRT plans are shown above and proton plans for the same patient seen below; (B) A pelvic sarcoma with comparative photon and proton plans shown in three planes.

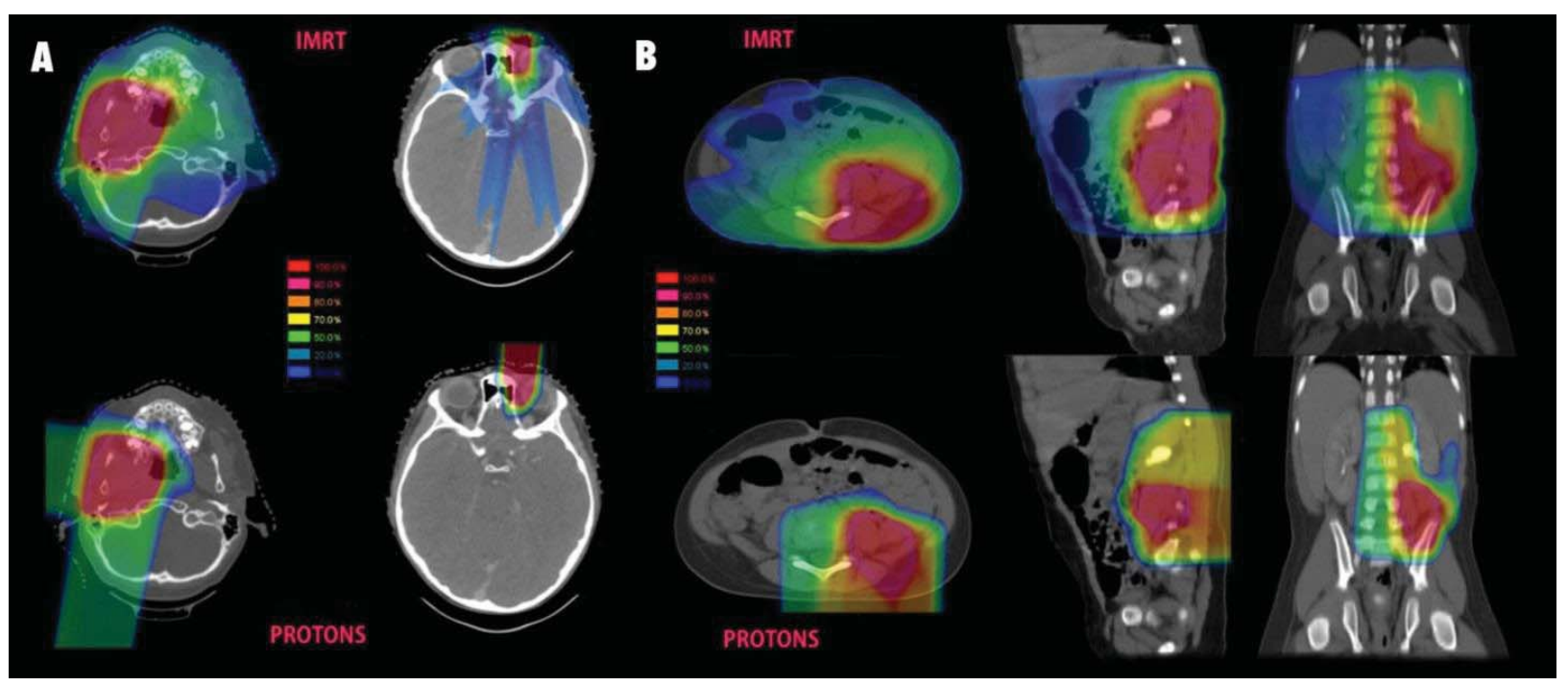

\section{Non-Rhabdomyosarcoma Soft Tissue Sarcoma}

Non-rhabdomyosarcoma soft tissue sarcomas (NRSTS) comprise about 4\% of childhood malignancies and affect approximately 500 children under the age of 20 years in the United States each year [6]. The estimated 5-year survival of children and adolescents with non-metastatic NRSTS is roughly $80 \%[23,24]$. The chance of cure is largely affected by surgical resectability, as children with grossly resected non-metastatic tumors have a 5 -year estimated survival of $89 \%$, compared with $56 \%$ for initially unresected tumors [25]. Radiation as an adjuvant is typically given to unresectable tumors, high-grade tumors less than $5 \mathrm{~cm}$ with involved surgical margins, or high-grade tumors greater than $5 \mathrm{~cm}$ regardless of margin status, and can be used pre-operatively with chemotherapy for delayed surgery in initially unresected disease. The doses of radiation used in the recently closed COG study for non-rhabdomyosarcomas ARST 0332 ranged from 45 Gy pre-operatively to 55.8 Gy for microscopic residual and 64.8 Gy for gross disease. Anthracycline based chemotherapy can be given for large, high-grade tumors or metastatic disease. A pooled analysis of the European and US experience with pediatric NRSTS from 1980-2005 demonstrated a significant survival advantage to adjuvant radiation after incomplete resection [26]. In the study published by Ferrari et al., the 5-year OS was $35 \%$ for those that did not receive radiation compared to $69 \%$ for those that did receive radiation, and this significance was retained on multivariate analysis.

Few studies of late effects in children and adolescents treated for NRSTS have been published and long-term quality of life and functional outcomes in this population are largely unknown. The 
recently closed COG protocol ARST 0332 a imed to reduce long-term toxicity by omitting chemotherapy and/or radiation in certain low to moderate risk presentations and will systematically evaluate the acute and long-term sequelae of treatment. Proton therapy was incorporated into the protocol and will be examined. Although to our knowledge there are no existing proton series dedicated to pediatric NRSTS, studies in adult paraspinal NRSTS treated with protons have found comparable local control and excellent toxicity profiles, and total integral dose and mean dose to the organs at risk were consistently shown to be reduced by a factor of 1.3 to 25 in dosimetric studies $[27,28]$.

\section{Osteosarcoma}

Within the United States, approximately 650-700 malignant bone tumors are diagnosed in patients under 20 years, of which 400 (56\%) are osteosarcoma (OSA) [29]. Traditionally, osteosarcoma has been viewed as a radioresistant tumor [30] and radiation has been used most successfully in the adjuvant and palliative setting [31]. A role for post-operative radiation in OSA of the head and neck, where gross total resection is frequently unattainable, was demonstrated by Guadagnolo et al. in their review of 119 adult and pediatric patients treated at MDACC with photon RT [32]. In the study, 92 (77\%) patients underwent surgery alone, and 27 (23\%) were treated with surgery and radiation (mean dose $=60 \mathrm{~Gy}$ ). Compared to surgery alone, RT improved 5-year OS ( $80 \%$ vs. $31 \%$ ), DSS ( $80 \%$ vs. $35 \%$ ) and LC (75\% vs. $24 \%)$ for patients with positive or uncertain resection margins. Despite improvements with adjuvant RT, local control still remains a significant problem and survival after local failure is typically as poor as with metastatic disease. Therefore, there exists great interest in using charged particle therapy to escalate dose to residual tumor and/or resection bed.

The largest experience with protons and osteosarcoma comes from a retrospective series of 55 patients treated at MGH between 1983 to 2009 with proton therapy or mixed photon-proton radiotherapy [33]. This cohort included pediatric and adult patients with a median age of 29 years (range, 2 to 76 years) with OSA of the pelvis, spine, ribs, skull, and facial bones. Most patients had positive surgical margins (49.1\%) following surgery and the remainder had biopsy only or minor a resection with residual gross tumor. Radiation dose ranged from 50.4 Gy to $80 \mathrm{~Gy} ; 40 \%$ patients received a dose between $60 \mathrm{~Gy}$ and $70 \mathrm{~Gy}$ and $50.1 \%$ received a total dose of $\geq 70 \mathrm{~Gy}$. Only 11 patients $(20 \%)$ received proton only RT and the majority of these had primary tumors of the skull and face. Preoperative RT was used in $13 \%$ of cases with a dose of $19.8 \mathrm{~Gy}$. MGH often utilizes a low dose pre-operative regimen with the goal of reducing intraoperative tumor seeding while protecting post-operative wound healing. All patients received neoadjuvant anthracycline based chemotherapy.

Clinical outcomes were excellent with 3 a nd 5 year local control rates of $82 \%$ and $72 \%$ respectively. Twelve patients failed locally and 10 were within the treatment field while two were considered marginal failures. Of those that recurred locally, 67\% were tumors arising from bones of the skull and on multivariate analysis, skull primaries were associated with a hazard ratio of 2.6. At 5 years, DFS was $65 \%$ and OS rate was $67 \%$. With regard to late effects, 17 patients $(31 \%)$ developed grade 3 or 4 toxicity and in 10 patients $(24 \%)$ the late effects were attributable to radiation. Grade 3 toxicity consisted of severe pain $(n=3)$, diplopia $(n=1)$, immobility of limb $(n=2)$, severe bowel dysfunction due to denervation $(n=1)$, and severe headaches $(n=1)$. Grade 4 toxicity, 
defined as a loss of organ or organ function, was seen in nine patients. Enucleation after RT was required in four patients, one patient suffered from ipsilateral loss of vision and orbital pain, and one patient suffered from ipsilateral hearing loss and blindness. Two patients were severely handicapped because of immobility or impairment of gait. One patient suffered from extensive structural alteration of the maxillary bone needing repeated adaptation of the prosthesis. Second malignancy was seen in two patients; one patient developed acute lymphocytic leukemia a year and a half after treatment, and the other patient died from a secondary squamous cell carcinoma of the maxilla 16 years after completion of therapy.

In comparing outcomes from this study to prior OSA outcomes published by MGH, the 5-year LC improved from $68 \%$ to $72 \%$, despite retaining 40 of the original patients in both studies [34]. The improvement was attributed to the higher RT dose used in the more recent patients and suggests that dose escalation allowed by proton RT will continue to play a role in the advancement of OSA treatment. Further, a meta-analysis done by the authors showed that as doses reached $70 \mathrm{~Gy}$ or higher, the benefit of surgical resection prior to RT lost significance. With limited data available, the careful weighing of potential surgical morbidity against the potential late effects of high dose RT should be done before treatment decisions are made.

\section{Ewing Sarcoma}

Ewing sarcoma represents a promising target for improving patient outcomes using proton RT. With 200 cas es per year in the US, it is the second most common primary bone tumor after osteosarcoma and comprises $34 \%$ of pediatric bone tumors and 3\% of all childhood cancers. The majority of patients (approximately 85\%) present with primary bone tumors but isolated soft tissue tumors are also observed. Primary sites typically include the pelvic bones $(23 \%)$, femur (18\%), ribs $(13 \%)$, tibia (10\%) and humerus (7\%), but tumors can arise from the head, neck, and skull as well. Radiation is used in conjunction with chemotherapy in $60 \%$ of Ewing's cases, either after resection for close/involved margins or as definitive therapy in unresectable disease [35-39]. With 5 year survival for localized disease at an upwards of $60 \%-70 \%$, reduction of late toxicity to normal tissue has become a major investigational goal [40-45]. Further, the need for doses up to 60 Gy and the frequency of primary sites in the pelvis, head and neck, base of skull, and spine make protons an ideal modality in young patients.

The only existing proton series dedicated to pediatric Ewing sarcoma was recently published by Rombi et al. The authors retrospectively reviewed 30 ped iatric Ewing cases treated with protons between 2003 and 2009 [46]. Most cases arose in the spine $(n=14)$ with other sites including pelvic $(n=4)$, head and neck/orbital $(n=4)$, base of skull $(n=3)$, cranial $(n=4)$ and chest wall $(n=1)$. Gross total resection or near total resection was achieved in only two patients and the majority of patients (17/30) had a biopsy only. Median follow up was 38.4 months all patients received proton RT (median dose $54 \mathrm{~Gy}$, range 45-58 Gy) and chemotherapy. The 3-year rates of LC, EFS, and OS were $86 \%, 60 \%$, and $89 \%$, respectively. Late sequelae seen consisted of five patients with scoliosis/kyphosis (three mild, one moderate, one severe) and all five had vertebral body primaries with laminectomy prior to RT. One patient with an orbital tumor developed epiphora from canicular stenosis and a left lid lag requiring gold seed placement after surgery and RT, and a s econd orbital patient developed a corneal ulcer. Endocrine deficiency was seen in two patients treated 
to cranial/base of skull tumors and high frequency hearing loss was seen in one patient with a head and neck $\mathrm{t}$ umor. Four patients also developed secondary hematologic malignancies attributable to chemotherapy.

MGH has also reported outcomes for two patients with Ewing sarcoma involving the sinonasal cavity and anterior skull base [47] and MDACC included one pelvic Ewing patient in adosimetric review of protons [22].

\section{Chordoma and Chondrosarcoma}

Chordomas and chondrosarcomas most commonly arise in adults, with a peak incidence in the fourth and fifth decade $[48,49]$. Fewer than $5 \%$ of these tumors are diagnosed in patients under 20 years of age. In children, chordomas arise more commonly in the spheno-occipital region, rather than the sacral predominance seen in adults, but can be found throughout the spine and sacrum [50]. Chordomas in the pediatric population can behave more aggressively than in adults, presenting with a shorter history of symptoms, a shorter interval to progression after surgery, and a higher rate of metastatic disease, especially in those under 5 years of age $[51,52]$. Whereas chordomas arise from notochord remnants within the axial skeleton, chondrosarcomas originate from cartilaginous elements within bone and are seen most often in the pelvis and femur but can occur in any number of locations, including the base of skull. The rates of long-term local control following treatment with surgery and radiation are significantly higher with chondrosarcoma than chordoma, ranging from $85 \%-100 \%$, and systemic therapy can be used in select situations [53-55].

Management of chordoma and chondrosarcoma relies heavily upon surgery as primary treatment. Despite advances in microscopic and image-guided neurosurgical procedures, complete resection in these tumors is achieved in only $50 \%-70 \%$ of cases, depending on location. Even with complete surgical resection, local recurrence ranges from $40 \%-60 \%$ [56-58]. Following surgery, RT is indicated for the presence of gross or microscopic residual disease, or in cases where violation of the tumor capsule has occurred and there is concern for contamination. Radiation combined with surgery has been shown to reduce local recurrence rates and proton RT has repeatedly demonstrated the ability to safely treat adult skull base patients and achieve higher local control than photon RT $[59,60]$. In a systematic review of all the major published chordoma series, the benefit of proton RT was demonstrated with an overall 5 year LC/OS of 36\%/54\% for photons $v s .64 \% / 80 \%$ for protons [59]. This benefit of RT has also been demonstrated in pediatric photon series and there is some data to suggest children have improved responses to radiation compared to their adult counterparts [51,61].

The published data for the use of protons with chordoma and chondrosarcoma is mainly limited to cranio-cervical disease. Halbrand et al. published their experience with 30 pediatric patients from l'Institut Curie in Orsay, France [62]. Children with tumors of the base of skull and cervical canal (26 chordoma and 4 chondrosarcoma), were treated with combination photon-proton RT following incomplete surgical resection. The mean total dose was $68.3 \mathrm{~Gy}$ although a cone down to the GTV was made after $55 \mathrm{~Gy}$. The maximum dose constraints were maintained at $\leq 56$ Gy to the optic nerves and optic chiasm, $\leq 64$ Gy to the anterior aspect of the brain stem, and at $\leq 56$ Gy to the mid-brainstem. Mean follow up was 26.5 months (range, 5-102 months). Local control was 83\% with all five failures seen in chordoma patients. Three of the failures were in field, one was a 
marginal failure, and one patient experienced seeding along the surgical path. The 5 year OS/PFS was $100 \% / 100 \%$ for chondrosarcoma and $81 \% / 77 \%$ for chordoma. Toxicity was remarkably low in this series with no child experiencing greater than grade $2 \mathrm{CTCAE}$ acute toxicity. Late toxicity consisted of unilateral hearing loss in one patient and grade 2 endocrine dysfunction in seven patients. Four patients experienced unilateral blindness yet all had reported this lack of vision prior to beginning RT.

Hug et al. reported a series of pediatric skull base tumors treated with protons at MGH and Loma Linda that included 10 chordomas and 3 chondrosarcomas, followed for a median of 40 months [63]. Recurrent disease was treated in 6 patients and all patients had evidence of gross residual disease at the time of radiation. All patients underwent resection or biopsy prior to RT. A dose of 78.6 Gy for chordoma and 70 Gy for chondrosarcoma was attempted, although the median dose for the chordoma cohort was $73.7 \mathrm{~Gy}$. More than half of these patients were treated with a mixed photon/proton plan. Normal tissue dose constraints were 60 Gy maximum to optic nerves and optic chiasm, 64 Gy to the brainstem and spinal cord surface, and $53 \mathrm{~Gy}$ to brainstem and spinal cord center. Local control was $60 \%$ and overall survival was $60 \%$ for the chordoma patients. The chondrosarcomas again faired better with all three patients achieving a local control and an overall survival of $100 \%$. No correlation was found between outcome and tumor size, duration of symptoms, or treatment for primary $v s$. recurrent disease, but female patients did have a statistically significant increased rate of progression. Acute effects from treatment were generally related to epilation and mucositis and all resolved after treatment completion. One patient did developed severe headaches within 4 weeks of proton RT and required analgesic treatment with resolution after several weeks. Severe late effects were seen in one chordoma patient after 75.4 Gy who developed cerebellar and brainstem parenchymal damage 16 months after treatment, resulting in right-sided motor weakness and ataxia. The authors note that this patient had three separate neurosurgical resections for progressive disease prior to radiation and that patients with more than two procedures prior to RT have been shown to demonstrate a higher rate of radiation induced brainstem damage [64].

The MGH later updated their pediatric series in a clinic-pathologic study published by Hoch et al. in 2006 to include 73 base of skull chordoma patients [52]. With a median follow up of 7.25 years, the overall survival rate was reported to be $81 \%$ for patients treated with surgery and protons or a combined proton/photon plan. It was noted by the authors that the 5 -year survival rate for a similar cohort of 125 adults with base of skull chordomas treated at MGH with surgery and proton beam radiation was 55\% [65], potentially illustrating the possibility of improved response to RT in pediatric patients. It should be pointed out that two institutions have subsequently published adult series combining surgery and proton RT for base of skull chordomas with 3 and 5 year survival rates of greater than $80 \%$, though the number of patients in each series were much fewer $[66,67]$. The authors also performed an in depth histopathologic review of the individual tumors and divided them into "conventional", "chondroid", "cellular", or "poorly differentiated" subtypes and reported the results. Of those who eventually died of disease, 14\% were conventional chordoma, 18\% were chondroid chordoma, $83 \%$ were poorly differentiated chordoma, and none were cellular chordoma. Further the average age of those with poorly differentiated chordoma was significantly younger than the rest of the cohort (4.8 years), and was hypothesized to account for the poor outcomes seen with pediatric chordomas under the age of 5 years. In the pediatric age group, metastatic disease has been 
documented to occur in up to $57.9 \%$ of patients under 5 years of age compared with only $8.5 \%$ of children 5 years or older, which the authors again hypothesize could be related to the prevalence of poorly differentiated histology.

Similar results for were reported by Rutz et al. at the Paul Scherrer Institute in Switzerland for 10 pediatric patients (six chordomas, four chondrosarcomas) that included extracranial tumors [67]. Tumors treated were found in the brain $(n=1)$, skull base $(n=5)$, cervical spine $(n=3)$, and lumbar spine $(n=1)$. Complete resection was achieved in $3 / 10$ children. Proton dose was 74.0 Gy for chordoma and 63.2-68.0 Gy for chondrosarcoma (median, 66.0 Gy), depending on histopathological grading and concurrent chemotherapy. Median follow-up time was 36 months (range, 8-77 months) and all patients were alive at last follow up without local or distant recurrence. Two patients experienced mild late adverse events with the first patient having neurosensory changes, alopecia and hypoaccusis and the second patient having grade 2 pituitary insufficiency.

\section{Conclusions}

Currently, there is sufficient data to suggest that in most pediatric cancers, sarcoma and otherwise, proton beam radiation delivers plans with superior dosimetric properties, specifically a reduced integral dose and reduced dose to organs at risk. Initial clinical data with protons and soft tissue sarcoma appears to show either equivalence or improvement in outcomes when compared to historical photon controls, and toxicity may be reduced. In the setting of bone and cartilaginous sarcomas a clearer advantage exists, mainly due to the ability to increase total dose while respecting the normal tissue tolerance of adjacent structures. Direct comparisons of proton and photon toxicity are plagued by historical differences in surgical technique, systemic therapy, and radiation delivery, as well as by institutional differences in the collection and scoring of toxicity data. The relatively recent incorporation of proton therapy into the majority of the COG protocols will likely provide the most accurate and applicable assessment of proton benefit. Further, almost all children with sarcoma treated with protons are now being enrolled on institution specific clinical and quality of life protocols, and long-term data will continue to emerge in the coming years. As more centers acquire proton capabilities and comparative costs drop, it is reasonable to expect widespread adoption of protons in a majority of pediatric sarcomas.

\section{Acknowledgments}

The authors would like to thank Amy Cook for her help with graphics and design.

\section{Conflicts of Interest}

The authors have no conflicts of interest to report.

\section{References}

1. Schwartz, C.L. Long-term survivors of childhood cancer: The late effects of therapy. Oncologist 1999, 4, 45-54. 
2. Goitein, M.; Jermann, M. The relative costs of proton and x-ray radiation therapy. Clin. Oncol. 2003, 15, S37-S50.

3. Pagnetti, H.; Niemierko, A.; Ancukiewitz, M.; Gerweck, L.E.; Goitein, M.; Loeffler, G.S.; Suit, H.D. Relative biological effectiveness (RBE) values for proton beam radiotherapy. Int. J. Radiat. Oncol. Biol. Phys. 2002, 53, 407-421.

4. Lomax, A.J.; Bortfeld, T.; Goitein, G.; Debus, J.; Dykstra, C.; Tercier, P.A.; Coucke, P.A.; Mirimanoff, R.O. A treatment planning inter-comparison of proton and intensity modulated photon radiotherapy. Radiother. Oncol. 1999, 51, 257-271.

5. Miralbell, R.; Lomax, A.; Cell, C.; Schneider, U. Potential reduction of the incidence of radiation induced second cancers by using proton beams in the treatment of pediatric tumors. Int. J. Radiat. Oncol. Biol. Phys. 2002, 54, 824-829.

6. Ries, L.A., Smith, M.A., Gurney, J.G., Linet, M., Tamra, T., Young, J.L., Bunin, G.R., Eds. Cancer Incidence and Survival among Children and Adolescents: United States SEER Program 1975-1995, National Cancer Institute, SEER Program; NIH Publication: Bethesda, MD, USA, 1999; No. 99-4649.

7. Pappo, A.S.; Shapiro, D.N.; Crist, W.M.; Maurer, H.M. Biology and therapy of pediatric rhabdomyosarcoma. J. Clin. Oncol. 1995, 13, 2123-2139.

8. Raney, R.B.; Anderson, J.R.; Barr, F.G.; Donaldson, S.S.; Pappo, A.S.; Qualman, S.J.; Wiener, E.S.; Maurer, H.M.; Crist, W.M. Rhabdomyosarcoma and undifferentiated sarcoma in the first two decades of life: A selective review of intergroup rhabdomyosarcoma study group experience and rationale for Intergroup Rhabdomyosarcoma Study V. J. Pediatr. Hematol. Oncol. 2001, 23, 215-220.

9. Ladra, M.; Szymonifka, J.; MacDonald, S.H.; Yong Yeap, B.; Friedmann, A.M.; Kasper, H.; Mahajan, A.; Grosshans, D.; Tarbell, N.; Yock, T.I. Proton Radiation Therapy for Rhabdomyosarcoma: Preliminary Results From a Multicenter Prospective Study. Int. J. Radiat. Oncol. Biol. Phys. 2013, 87, S597-S598.

10. Crist, W.M.; Anderson, J.R.; Meza, J.L.; Fryer, C.; Raney, R.B.; Ruymann, F.B.; Breneman, J.; Qualman, S.J.; Wiener, E.; Wharam, M.; et al. Intergroup rhabdomyosarcoma study-IV: Results for patients with nonmetastatic disease. J. Clin. Oncol. 2001, 19, 3091-3102.

11. Arndt, C.A.; Stoner, J.A.; Hawkins, D.S.; Rodeberg, D.A.; Hayes-Jordan, A.A.; Paidas, C.N.; Parham, D.M.; Teot, L.A.; Wharam, M.D.; Breneman, J.C.; et al. Vincristine, actinomycin, and cyclophosphamide compared with vincristine, actinomycin, and cyclophosphamide alternating with vincristine, topotecan, and cyclophosphamide for intermediate-risk rhabdomyosarcoma: Children's oncology group study D9803. J. Clin. Oncol. 2009, 27, 5182-5188.

12. Pappo, A.S.; Lyden, E.; Breitfeld, P.; Donaldson, S.S.; Wiener, E.; Parham, D.; Crews, K.R.; Houghton, P.; Meyer, W.H. Two consecutive phase II window trials of irinotecan alone or in combination with vincristine for the treatment of metastatic rhabdomyosarcoma: The Children's Oncology Group. J. Clin. Oncol. 2007, 25, 362-369.

13. Childs, S.K.; Kozak, K.R.; Friedmann, A.M.; Yeap, B.Y.; Adams, J.; Macdonald, S.M.; Liebsch, N.J.; Tarbell, N.J.; Yock, T.I. Proton radiotherapy for parameningeal rhabdomyosarcoma: Clinical outcomes and late effects. Int. J. Radiat. Oncol. Biol. Phys. 2011, $82,635-642$. 
14. Paulino, A.C.; Simon, J.H.; Zhen, W.; Wen, B.C. Long-term effects in children treated with radiotherapy for head and neck rhabdomyosarcoma. Int. J. Radiat. Oncol. Biol. Phys. 2000, 48, 1489-1495.

15. Raney, R.B.; Asmar, L.; Vassilopoulou-Sellin, R.; Klein, M.J.; Donaldson, S.S.; Green, J.; Heyn, R.; Wharam, M.; Glicksman, A.S.; Gehan, E.A.; et al. Late complications of therapy in 213 children with localized, nonorbital soft-tissue sarcoma of the head and neck: A descriptive report from the Intergroup Rhabdomyosarcoma Studies (IRS)-II and -III. Med. Pediatr. Oncol. 1999, 33, 362-371.

16. Kozak, K.R.; Adams, J.; Krejcarek, S.J.; Tarbell, N.J.; Yock, T.I. A dosimetric comparison of proton and intensity-modulated photon radiotherapy for pediatric parameningeal rhabdomyosarcomas. Int. J. Radiat. Oncol. Biol. Phys. 2009, 74, 179-186.

17. Merchant, T.E.; Rose, S.R.; Bosley, C.; Wu, S.; Xiong, X.; Lustig, R.H. Growth hormone secretion after conformal radiation therapy in pediatric patients with localized brain tumors. J. Clin. Oncol. 2011, 29, 4776-4780.

18. Yock, T.; Schneider, R.; Friedmann, A.; Adams, J.; Fullerton, B.; Tarbell, N. Proton radiotherapy for orbital rhabdomyosarcoma: Clinical outcome and a dosimetric comparison with photons. Int. J. Radiat. Oncol. Biol. Phys. 2005, 63, 1161-1168.

19. Heyn, R.; Ragab, A.; Raney, R.B., Jr.; Ruymann, F.; Tefft, M.; Lawrence, W., Jr.; Soule, E.; Maurer, H.M. Late effects of therapy in orbital rhabdomyosarcoma in children: A report from the Intergroup Rhabdomyosarcoma Study. Cancer 1986, 57, 1738-1743.

20. Wolden, S.L.; Wexler, L.H.; Kraus, D.H.; Laquaglia, M.P.; Lis, E.; Meyers, P.A. Intensity-modulated radiotherapy for head-and-neck rhabdomyosarcoma. Int. J. Radiat. Oncol. Biol. Phys. 2005, 61, 1432-1438.

21. Cotter, S.E.; Herrup, D.A.; Friedmann, A.; Macdonald, S.M.; Pieretti, R.V.; Robinson, G.; Adams, J.; Tarbell, N.J.; Yock, T.I. Proton radiotherapy for pediatric bladder/prostate rhabdomyosarcoma: Clinical outcomes and dosimetry compared to intensity-modulated radiation therapy. Int. J. Radiat. Oncol. Biol. Phys. 2011, 81, 1367-1373.

22. Lee, C.T.; Bilton, S.D.; Famiglietti, R.M.; Riley, B.A.; Mahajan, A.; Chang, E.L.; Maor, M.H.; Woo, S.Y.; Cox, J.D.; Smith, A.R. Treatment planning with protons for pediatric retinoblastoma, medulloblastoma, and pelvic sarcoma: How do protons compare with other conformal techniques? Int. J. Radiat. Oncol. Biol. Phys. 2005, 63, 362-372.

23. Spunt, S.L.; Hill, D.A.; Motosue, A.M.; Billups, C.A.; Cain, A.M.; Rao, B.N.; Pratt, C.B.; Merchant, T.E.; Pappo, A.S. Clinical features and outcome of initially unresectednonmetastatic pediatric nonrhabdomyosarcoma soft tissue sarcoma. J. Clin. Oncol. 2002, 20, 3225-3235.

24. Spunt, S.L.; Poquette, C.A.; Hurt, Y.S.; Cain, A.M.; Rao, B.N.; Merchant, T.E.; Jenkins, J.J.; Santana, V.M.; Pratt, C.B.; Pappo, A.S. Prognostic factors for children and adolescents with surgically resected nonrhabdomyosarcoma soft tissue sarcoma: An analysis of 121 pat ients treated at St Jude Children's Research Hospital. J. Clin. Oncol. 1999, 17, 3697-3705.

25. Pappo, A.S.; Rao, B.N.; Jenkins, J.J.; Merchant, T.; Poquette, C.A.; Cain, A.; Pratt, C.B. Metastatic nonrhabdomyosarcomatous soft-tissue sarcomas in children and adolescents: The St. Jude Children's Research Hospital experience. Med. Pediatr. Oncol. 1999, 33, 76-82. 
26. Ferrari, A.; Miceli, R.; Rey, A.; Oberlin, O.; Orbach, D.; Brennan, B.; Mariani, L.; Carli, M.; Bisogno, G.; Cecchetto, G.; et al. Non-metastatic unresectedpaediatric non-rhabdomyosarcoma soft tissue sarcomas: Results of a poo led analysis from United States and European groups. Eur. J. Cancer 2011, 47, 724-731.

27. Weber, D.C.; Trofimov, A.V.; Delaney, T.F.; Bortfeld, T. A treatment planning comparison of intensity modulated photon and proton therapy for paraspinal sarcomas. Int. J. Radiat. Oncol. Biol. Phys. 2004, 58, 1596-1606.

28. Weber, D.C.; Rutz, H.P.; Bolsi, A.; Pedroni, E.; Coray, A.; Jermann, M.; Lomax, A.J.; Hug, E.B.; Goitein, G. Spot scanning proton therapy in the curative treatment of adult patients with sarcoma: The Paul Scherrer institute experience. Int. J. Radiat. Oncol. Biol. Phys. 2007, 69, $865-871$.

29. SEER Pediatric Monograph on Malignant Bone Tumors. Available online: http://seer.cancer.gov/publications/childhood/bone.pdf (accessed on 17 December 2013).

30. Ogawa, Y.; Takahashi, T.; Kobayashi, T.; Kariya, S.; Nishioka, A.; Hamasato, S.; Moriki, T.; Seguchi, H.; Yoshida, S.; Sonobe, H. Immunocytochemical characteristics of human osteosarcoma cell line HS-Os-1: Possible implication in apoptotic resistance against irradiation. Int. J. Mol. Med. 2004, 14, 397-403.

31. Mahajan, A.; Woo, S.Y.; Kornguth, D.G.; Hughes, D.; Huh, W.; Chang, E.L.; Herzog, C.E.; Pelloski, C.E.; Anderson, P. Multimodality treatment of osteosarcoma: Radiation in a high-risk cohort. Pediatr. Blood Cancer 2008, 50, 976-982.

32. Guadagnolo, B.A.; Zagars, G.K.; Raymond, A.K.; Benjamin, R.S.; Sturgis, E.M. Osteosarcoma of the jaw/craniofacial region: Outcomes after multimodality treatment. Cancer 2009, 115, $3262-3270$.

33. Ciernik, I.F.; Niemierko, A.; Harmon, D.C.; Kobayashi, W.; Chen, Y.L.; Yock, T.I.; Ebb, D.H.; Choy, E.; Raskin, K.A.; Liebsch, N.; et al. Proton-based radiotherapy for unresectable or incompletely resected osteosarcoma. Cancer 2011, 117, 4522-4530.

34. DeLaney, T.F.; Park, L.; Goldberg, S.I.; Hug, E.B.; Liebsch, N.J.; Munzenrider, J.E.; Suit, H.D. Radiotherapy for local control of osteosarcoma. Int. J. Radiat. Oncol. Biol. Phys. 2005, 61, 492-498.

35. Cotterill, S.J.; Ahrens, S.; Paulussen, M.; Jürgens, H.F.; Voûte, P.A.; Gadner, H.; Craft, A.W. Prognostic factors in Ewing's tumor of bone: Analysis of 975 patients from the European Intergroup Cooperative Ewing's Sarcoma Study Group. J. Clin. Oncol. 2000, 18, 3108-3114.

36. Donaldson, S.S.; Torrey, M.; Link, M.P.; Glicksman, A.; Gilula, L.; Laurie, F.; Manning, J.; Neff, J.; Reinus, W.; Thompson, E.; et al. A multidisciplinary study investigating radiotherapy in Ewing's sarcoma: End results of POG \#8346. Pediatric Oncology Group. Int. J. Radiat. Oncol. Biol. Phys. 1998, 42, 125-135.

37. Shankar, A.G.; Pinkerton, C.R.; Atra, A.; Ashley, S.; Ashley, S.; Lewis, I.; Spooner, D.; Cannon, S.; Grimer, R.; Cotterill, S.J.; Craft, A.W.; et al. Local therapy and other factors influencing site of relapse in patients with localised Ewing's sarcoma. United Kingdom Children's Cancer Study Group (UKCCSG). Eur. J. Cancer 1999, 35, 1698-1704. 
38. Elomaa, I.; Blomqvist, C.P.; Saeter, G.; Akerman, M.; Stenwig, E.; Wiebe, T.; Björk, O.; Alvegård, T.A. Five-year results in Ewing's sarcoma. The Scandinavian Sarcoma Group experience with the SSG IX protocol. Eur. J. Cancer 2000, 36, 875-880.

39. Schuck, A.; Ahrens, S.; Paulussen, M.; Kuhlen, M.; Könemann, S.; Rübe, C.; Winkelmann, W.; Kotz, R.; Dunst, J.; Willich, N.; et al. Local therapy in localized Ewing tumors: Results of 1058 patients treated in the CESS 81, CESS 86, and EICESS 92 trials. Int. J. Radiat. Oncol. Biol. Phys. 2003, 55, 168-177.

40. Bacci, G.; Toni, A.; Avella, M.; Manfrini, M.; Sudanese, A.; Ciaroni, D.; Boriani, S.; Emiliani, E.; Campanacci, M. Long-term results in 144 localized Ewing's sarcoma patients treated with combined therapy. Cancer 1989, 63, 1477-1486.

41. Wilkins, R.M.; Pritchard, D.J.; Burgert, E.O., Jr.; Unni, K.K. Ewing's sarcoma of bone. Experiences with 140 patients. Cancer 1986, 58, 2551-2555.

42. Burgert, E.O., Jr.; Unni, K.K. The role of radiation therapy in the management of nonmetastatic Ewing's sarcoma of bone. Report of the Intergroup Ewing's sarcoma study. Int. J. Radiat. Oncol. Biol. Phys. 1981, 7, 141-145.

43. Jurgens, H.; Bier, V.; Dunst, J.; Harms, D.; Jobke, A.; Kotz, R.; Kühl, J.; Müller-Weihrich, S.; Ritter, J.; Salzer-Kuntschik, M.; et al. The German Society of Pediatric Oncology Cooperative Ewing Sarcoma Studies CESS 81/86: Report after 6 1/2 years. Klin. Padiatr. 1988, 200, $243-252$.

44. Dunst, J.; Sauer, R.; Burgers, J.M.; Hawliczek, R.; Kürten, R.; Müller, R.P.; Wannenmacher, M.; Jürgens, H. Radiotherapy in ewing sarcoma: Current results of the german society of pediatric oncology studies CESS 81 and CESS 86. Klin. Padiatr. 1988, 200, 261-266.

45. Jurgens, H.; Exner, U.; Gardner, H.; Harms, D.; Michaelis, J.; Sauer, R.; Treuner, J.; Voûte, T.; Winkelmann, W.; Winkler, K.; et al. Multidisciplinary treatment of primary Ewing's sarcoma of bone. A 6-year experience of a European cooperative trial. Cancer 1988, 61, 23-32.

46. Rombi, B.; Delaney, T.F.; Macdonald, S.M.; Huang, M.S.; Ebb, D.H.; Liebsch, N.J.; Raskin, K.A.; Yeap, B.Y.; Marcus, K.J.; Tarbell, N.J.; et al. Proton radiotherapy for pediatric Ewing's sarcoma: Initial clinical outcomes. Int. J. Radiat. Oncol. Biol. Phys. 2011, 82, $1142-1148$.

47. Gray, S.T.; Chen, Y.L.; Lin, D.T. Efficacy of proton beam therapy in the treatment of Ewing's sarcoma of the paranasal sinuses and anterior skull base. Skull Base 2009, 19, 409-416.

48. Cummings, B.J.; Hodson, D.I.; Bush, R.S. Chordoma: The results of megavoltage radiation therapy. Int. J. Radiat. Oncol. Biol. Phys. 1983, 9, 633-642.

49. Heffelfinger, M.J.; Dahlin, D.C.; MacCarty, C.S.; Beabout, J.W. Chordomas and cartilaginous tumors at the skull base. Cancer 1973, 32, 410-420.

50. Wold, L.E.; Laws, E.R., Jr. Cranial chordomas in children and young adults. J. Neurosurg. 1983, 59, 1043-1047.

51. Borba, L.A.B.; Al-Mefty, O.; Mrak, R.E.; Suen, J. Cranialchordomas in children and adolescents. J. Neurosurg. 1996, 84, 584-591.

52. Hoch, B.L.; Nielsen, G.P.; Liebsch, N.J.; Rosenberg, A.E. Base of skull chordomas in children and adolescents: A clinicopathologic study of 73 cases. Am. J. Surg. Pathol. 2006, 30, 811-818. 
53. Noel, G.; Harbrand, J.L.; Jauffret, E. Radiation therapy for chordoma and chondrosarcoma of the skull base and the cervical spine. Strahlenther. Onkol. 2003, 179, 241-247.

54. Hug, E.B.; Slater, J.D. Proton radiotherapy for chordomas and chondrosarcomas of the skull base. Neurosurg. Clin. N. Am. 2000, 11, 627-634.

55. Schulz-Ertner, D.; Nikoghosyan, A.; Thilmann, C.; Haberer, T.; Jäkel, O.; Karger, C.; Kraft, G.; Wannenmacher, M.; Debus, J. Results of carbon ion radiotherapy in 152 patients. Int. J. Radiat. Oncol. Biol. Phys. 2004, 58, 631-640.

56. Tzortzidis, F.; Elahi, F.; Wright, D.; Natarajan, S.K.; Sekhar, L.N. Patient outcome at long-term follow-up after aggressive microsurgical resection of cranial base chordomas. Neurosurgery 2006, 59, 230-237.

57. Boriani, S.; Bandiera, S.; Biagini, R.; Bacchini, P.; Boriani, L.; Cappuccio, M.; Chevalley, F.; Gasbarrini, A.; Picci, P.; Weinstein, J.N. Chordoma of the mobile spine: Fifty years of experience. Spine 2006, 31, 493-503.

58. Fuchs, B.; Dickey, I.D.; Yaszemski, M.J.; Inwards, C.Y.; Sim, F.H. Operative management of sacral chordoma. J. Bone Joint Surg. Am. 2005, 87, 2211-2216.

59. Munzenrider, J.E.; Liebsch, N.J. Proton therapy for tumors of the skull base. Strahlenther. Onkol. 1999, 175, 57-63.

60. Hug, E.B.; Loredo, L.N.; Slater, J.D.; DeVries, A.; Grove, R.I.; Schaefer, R.A.; Rosenberg, A.E.; Slater, J.M. Proton radiation therapy for chordomas and chondrosarcomas of the skull base. J. Neurosurg. 1999, 91, 432-439.

61. Montgomery, A.H.; Wolman, I.J. Sacrococcy gealchordomas in children. Am. J. Dis. Child. 1973, 46, 1263-1281.

62. Habrand, J.L.; Schneider, R.; Alapetite, C.; Feuvret, L.; Petras, S.; Datchary, J.; Grill, J.; Noel, G.; Helfre, S.; Ferrand, R.; et al. Proton therapy in pediatric skull base and cervical canal low-grade bone malignancies. Int. J. Radiat. Oncol. Biol. Phys. 2008, 71, 672-675.

63. Hug, E.B.; Sweeney, R.A.; Nurre, P.M.; Holloway, K.C.; Slater, J.D.; Munzenrider, J.E. Proton radiotherapy in management of pediatric base of skull tumors. Int. J. Radiat. Oncol. Biol. Phys. 2002, 52, 1017-1024.

64. Debus, J.; Hug, E.B.; Liebsch, N.J.; O’Farrel, D.; Finkelstein, D.; Efird, J.; Munzenrider, J.E. Brainstem tolerance to conformal radiotherapy of skull base tumors. Int. J. Radiat. Oncol. Biol. Phys. 1997, 39, 967-975.

65. Nielsen, G.P.; Rosenberg, A.E.; Liebsch, N.J. Chordomas of the base of skull in children and adolescents: A clinicopathologic study of 35 cases. Mod. Pathol. 1996, 9, 11A.

66. Yasuda, M.; Bresson, D.; Chibbaro, S.; Cornelius, J.F.; Polivka, M.; Feuvret, L.; Takayasu, M.; George, B. Chordomas of the skull base and cervical spine: Clinical outcomes associated with a multimodal surgical resection combined with proton-beam radiation in 40 patients. Neurosurg. Rev. 2012, 35, 171-182.

67. Rutz, H.P.; Weber, D.C.; Sugahara, S.; Timmermann, B.; Lomax, A.J.; Bolsi, A.; Pedroni, E.; Coray, A.; Jermann, M.; Goitein, G. Extracranialchordoma: Outcome in patients treated with function-preserving surgery followed by spot-scanning proton beam irradiation. Int. J. Radiat. Oncol. Biol. Phys. 2007, 67, 512-520. 
Reprinted from Cancers. Cite as: Tejani, M.A.; Galloway, T.J.; Lango, M.; Ridge, J.A.; von Mehren, M. Head and Neck Sarcomas: A Comprehensive Cancer Center Experience. Cancers 2013, 5, 890-900.

Article

\title{
Head and Neck Sarcomas: A Comprehensive Cancer Center Experience
}

\author{
Mohamedtaki A. Tejani ${ }^{1}$, Thomas J. Galloway ${ }^{2}$, Miriam Lango ${ }^{3}$, John A. Ridge ${ }^{3}$ and \\ Margaret von Mehren ${ }^{4, *}$
}

1 Division of Hematology/Oncology, University of Rochester Medical Center, Rochester, NY 14642, USA; E-Mail: mohamed_tejani@urmc.rochester.edu

2 Department of Radiation Oncology, Fox Chase Cancer Center, Philadelphia, PA 19111, USA; E-Mail: thomas.galloway@fccc.edu

3 Department of Surgical Oncology, Fox Chase Cancer Center, Philadelphia, PA 19111, USA; E-Mails: miriam.lango@fccc.edu (M.L.); drew.ridge@fccc.edu (J.A.R.)

4 Department of Medical Oncology, Fox Chase Cancer Center, Philadelphia, PA 19111, USA

* Author to whom correspondence should be addressed; E-Mail: margaret.vonmehren@fccc.edu; Tel.: +1-215-728-2814; Fax: +1-215-728-3639.

Received: 20 May 2013; in revised form: 20 May 2013 / Accepted: 18 June 2013 /

Published: 15 July 2013

\begin{abstract}
Head/neck sarcomas are rare, accounting for about $1 \%$ of head/neck malignancies and 5\% of sarcomas. Outcomes have historically been worse in this group, due to anatomic constraints leading to difficulty in completely excising tumors, with high rates of local recurrence. We retrospectively analyzed cases of head/neck soft tissue sarcomas (STS) and osteogenic sarcomas managed in a multi-disciplinary setting at Fox Chase Cancer Center from 1999-2009 to describe clinicopathologic characteristics, treatment, outcomes, and prognostic factors for disease control and survival. Thirty patients with STS and seven patients with osteogenic sarcoma were identified. Most STS were high grade (23) and almost all were localized at presentation (28). Common histologies were synovial cell (6), rhabdomyosarcoma (5), angiosarcoma (4), liposarcoma (4) and leiomyosarcoma (3). The type of primary therapy and disease outcomes were analyzed. Cox proportional hazards regression analysis was performed to identify predictors of disease-free survival (DFS) and overall survival (OS). The HR and 95\% CI for Cox model and median DFS/OS analyzed by Kaplan-Meier curves were calculated.
\end{abstract}


Keywords: head and neck sarcomas; soft tissue sarcomas; osteogenic sarcomas

\section{Introduction}

Head and neck sarcomas are rare tumors, accounting for only $1 \%$ of all head and neck malignancies and $5 \%$ of all sarcomas [1,2]. They comprise about 1,000 to 1,500 cases per year in the United States and the many histologic subtypes make rigorous study of these neoplasms difficult. Diagnostic and treatment algorithms are mainly based on expert consensus and retrospective case series. The experiences depend strongly on the reporting entity-patients predominantly seen by head and neck surgeons, pediatric oncologists and medical oncologists will have disparate histologies and clinical features. Our study focuses solely on adults treated in multi-disciplinary fashion by surgical, radiation and medical oncologists at Fox Chase Cancer Center.

In general, the natural history of these sarcomas parallels that of their non-head and neck counterparts, but with a higher reported rate of local recurrence and lower overall survival rate. In a single institution series of 102 patients with head and neck sarcomas between 1960 and 1999 [3], local control rates were inferior compared with non-head-and-neck primaries $(74 \%$ vs. $85 \%$, $p<0.001)$ as were disease-specific survival rates $(64 \% v s .76 \%, p<0.001)$. It is thought that worse disease control is a function of anatomic constraints limiting functional resections rather than a difference in biologic behavior and/or tumor histology. Osteosarcomas of the head and neck, compared with those presenting in long bones, arise in a somewhat older age group, have a higher risk for local recurrence, and a lower risk for distant metastasis [4].

We retrospectively analyzed Fox Chase Cancer Center's experience with head and neck sarcomas during the past 10 years. We characterized a cohort of 37 pat ients with respect to their clinicopathologic features and course. We focused in particular on the results of treatment and identifying prognostic factors for DFS and OS in the era of contemporary multidisciplinary management. In general, whenever possible, patients with soft tissue sarcomas of the head/neck at our institution are treated with complete surgical resection. Recommendations for adjuvant chemotherapy and/or radiation are then made based on discussion at a multi-disciplinary conference which takes into account all clinical and pathologic features of a par ticular tumor, including histology. On the other hand, patients with osteosarcomas of the head/neck usually receive pre-operative chemotherapy followed by surgical resection. Again, decisions regarding adjuvant therapy are made based on final tumor size, histology and margin status.

\section{Methods}

Eligible patients were identified in the Center's tumor registry. After receiving IRB approval, the medical records of all patients with head and neck sarcomas treated at Fox Chase Cancer Center between January 1999 and December 2009 were reviewed. The head and neck was defined as any site above the clavicles. Only patients whose diagnosis was confirmed by a pathology report were included in this study. Patients seen only for a second opinion, without treatment or follow-up at the Center, were excluded. 
We recorded demographic and clinicopathologic characteristics including age, gender, presenting symptoms, tumor site, size, AJCC and MSKCC stage, histology, grade, margin status (if applicable) and sites of recurrence/metastases. Tumor grade at our institution is based on the French Federation Nationale des Centres de L utte Contre le Cancer (FNCLCC) system. We a lso extracted data regarding treatment modality delivered (surgery, radiation and/or systemic therapy) at presentation and recurrence. Disease-free and overall survival were computed using the Kaplan-Meier method. DFS was measured starting on date of surgical resection and OS was measured starting on date of diagnostic biopsy. We were not able to accurately determine disease-specific survival (DSS) for our cohort since many patients were lost to follow-up after their initial treatment. Univariate analysis of clinicopathologic factors that could potentially affect survival were performed using the Cox proportional hazard model. The hazard ratio (HR) and 95\% confidence intervals (CI) were reported.

\section{Results for Soft Tissue Sarcoma Cohort}

\subsection{Patient and Tumor Characteristics}

Between January 1999 and December 2009, 34 patients with head and neck sarcomas were seen. For the purposes of this study, four patients were excluded since they were only seen once for consultation with no follow-up information. Age at diagnosis ranged between 15 and 91 (median, 50 years). There were 20 male and 10 female patients. Four patients had a history of prior radiation exposure to the head and neck region for preexisting conditions and one patient had Gorlin's syndrome (which is known to increase the risk of developing cancers).

Tumor site was grouped by the following anatomical regions: (a) scalp/face, (b) parotid/neck and (c) upper airway. Table 1 shows the patient distribution according to tumor site and histology. Most common were synovial cell sarcoma (6), rhabdomyosarcoma (5), angiosarcoma (4), liposarcoma (4) and leiomyosarcoma (3).

Table 1. Patient distribution according to tumor histology and site.

\begin{tabular}{lcccc}
\hline Histology & Scalp/Face & Parotid/Neck & Upper Airway & Total \\
\hline Synovial cell sarcoma & 3 & 3 & & 6 \\
Rhabdomyosarcoma & 4 & & 1 & 5 \\
Angiosarcoma & 4 & & & 4 \\
Liposarcoma & 1 & 3 & 1 & 4 \\
Leiomyosarcoma & 2 & & 1 & 3 \\
Spindle cell sarcoma & 1 & 1 & 1 & 3 \\
Alveolar soft part sarcoma & & & & 1 \\
Carcinosarcoma & 1 & & & 1 \\
Epithelioid sarcoma & 1 & & & 1 \\
Fibrosarcoma & 1 & & & 1 \\
Histiocytic sarcoma & 1 & 7 & 4 & 30 \\
\end{tabular}

Tumor size and grade were classified as per soft tissue sarcoma American Joint Committee on Cancer (AJCC) as well as Memorial Sloan Kettering Cancer Center (MSKCC) staging systems. 
Table 2 shows patient distribution according to these two parameters. 23 patients $(77 \%)$ had high grade disease. The majority ( 28 of 30 ) presented with locoregional disease and only three had nodal involvement (all of these were rhabdomyo sarcoma cases). Two patients had metastases at diagnosis (one case each of angiosarcoma and synovial cell).

Table 2. Patient distribution according to tumor size and grade.

\begin{tabular}{lccccc}
\hline \multirow{2}{*}{ Size } & \multicolumn{5}{c}{ Grade } \\
\cline { 2 - 6 } & Low & Intermediate & High & Not Specified & Total \\
\hline$<5 \mathrm{~cm}$ & 1 & 1 & 10 & 1 & 13 \\
$>5 \mathrm{~cm}$ & 1 & 1 & 8 & 1 & 11 \\
Unknown & & 1 & 5 & & 6 \\
Total & 2 & 3 & 23 & 2 & 30 \\
\hline
\end{tabular}

\subsection{Treatment}

Of 28 patients with localized disease, 24 underwent resection. In general, the surgeons performed an excision as wide as permitted by the nearby vital structures and functional concerns. Therefore, in this analysis, the surgical procedure is defined as gross total excision with final microscopic involved or R1 (7) and clear or R0 (17) margins. Seven patients underwent successful re-excision to achieve clear margins. Elective nodal dissection was not routinely performed in v iew of the low rate of regional node metastases in sarcomas. The four patients who did not have surgical excision included two angiosarcoma cases with locally advanced disease who received systemic chemotherapy and two rhabdomyosarcoma cases who were treated with combined modality chemotherapy and radiation.

Sixteen of 24 patients undergoing surgical resection also received radiation in a $\mathrm{n}$ adjuvant (post-operative) fashion. Total tumor doses ranged from 5,000 cGy to 6,600 cGy; most patients received around 6,000 cGy over 30 fractions. IMRT (intensity-modulated radiation therapy) was utilized in all patients. Treatment volume was designed to cover the primary site plus a generous margin. The neck was not treated electively. Of the eight patients who did not receive radiation, five had tumors well below $5 \mathrm{~cm}$ in size with clear resection margins while two patients had received prior radiation which precluded them from additional therapy and one patient recurred within one month of resection. The median time interval between surgery and post-operative radiation therapy was 12 weeks.

Chemotherapy was administered to six patients, after completing post-operative radiation, on an individualized basis. These patients either had rhabdomyosarcoma (three cases), which is known to be sensitive to chemotherapy, or high-risk resected tumors (three cases) based on size and involved margins. Standard vincristine, adriamycin, cytoxan (VAC) chemotherapy was used for rhabdomyosarcoma cases and Ifosfamide-based therapy was used for other high-risk tumors.

\subsection{Recurrence and Survival}

Tumor recurrence was documented in sixteen patients: Seven locoregionally and at distant metastatic sites, five in the locoregional area alone and four in distant metastatic sites only. Sites of metastases included lung, liver and bones. Only two cases involved regional lymph node metastasis (angiosarcoma and rhabodomyosarcoma) which is not atypical for these sarcoma histologies. Over 
ninety percent of recurrences (15 of 16) occurred within the first 3 years. Median DFS for our cohort was 1.1 years (95\% CI: 0.7-12.1) and median OS was 3.3 years (95\% CI: 2.1-5.4).

In the recurrent and metastatic setting, 18 pat ients underwent salvage therapy in various combinations. Nine patients were submitted to repeat surgical resection, $10 \mathrm{r}$ eceived palliative radiation and 17 received standard or protocol-based systemic therapy. For patients with local or distant relapse, the 5-year survival with salvage treatment was dismal, at $24 \%$ in our series. More patients with local failure achieved long-term survival than those with distant spread.

\subsection{Prognostic Factors}

Univariate analysis using Cox progression hazard model was performed using margin status, size $(<5 \mathrm{~cm} v s .>5 \mathrm{~cm})$, age at diagnosis $(<50$ vs. $>50)$, grade (low-intermediate $v s$. high) and multiple other recognized prognostic factors to identify predictors of DFS and OS. Patients with R1 resections had worse DFS (HR 3.74, 95\% CI: 0.98-14.34) and OS (HR 4.4, 95\% CI: 1.11-17.4). Tumors $>5 \mathrm{~cm}$ were also associated with worse DFS (HR 2.79, 95\% CI: 0.84-9.25). Age and grade did not correlate with outcome in our cohort. (See Table 3 and Figure 1).

Table 3. Univariate analysis of factors Associated WITH DFS and OS in soft tissue sarcomas.

\begin{tabular}{|c|c|c|c|c|c|c|}
\hline \multirow{2}{*}{ Variable } & \multirow{2}{*}{ Category } & \multirow{2}{*}{$\begin{array}{c}\text { Number } \\
\text { (n) }\end{array}$} & \multicolumn{2}{|c|}{ Disease-Free Survival } & \multicolumn{2}{|c|}{ Overall Survival } \\
\hline & & & HR $(95 \%$ CI $)$ & $p$-value & HR $(95 \%$ CI $)$ & $p$-value \\
\hline \multirow{2}{*}{ Margin status } & Clear & 17 & 1.0 & 0.05 & 1.0 & 0.03 \\
\hline & Involved & 7 & $3.74(0.98,14.34)$ & & $4.4(1.11,17.4)$ & \\
\hline \multirow{2}{*}{ Size } & $<5 \mathrm{~cm}$ & 13 & 1.0 & 0.09 & 1.0 & 0.38 \\
\hline & $>5 \mathrm{~cm}$ & 11 & $2.79(0.84,9.25)$ & & $1.63(0.54,4.9)$ & \\
\hline \multirow{2}{*}{$\begin{array}{c}\text { Age at } \\
\text { diagnosis }\end{array}$} & $<50$ & 12 & 1.0 & 0.77 & 1.0 & 0.3 \\
\hline & $>50$ & 18 & $0.85(0.29,2.5)$ & & $1.73(0.64,4.66)$ & \\
\hline \multirow{2}{*}{$\begin{array}{c}\text { FNCLCC } \\
\text { Grade }\end{array}$} & Lowintermed & 5 & & & & \\
\hline & high & 23 & - & NS & - & NS \\
\hline \multirow{2}{*}{ Gender } & Male & 20 & 1.0 & 0.16 & 1.0 & 0.18 \\
\hline & Female & 10 & $0.46(0.16,1.35)$ & & $0.51(0.19,1.37)$ & \\
\hline \multirow{3}{*}{ Site } & Scalp/face & 19 & 1.0 & 0.31 & 1.0 & 0.97 \\
\hline & Parotid/neck & 7 & $0.77(0.20,2.93)$ & & $0.61(0.17,2.18)$ & \\
\hline & Upper Airway & 4 & $2.40(0.69,8.32)$ & & $1.32(0.36,4.78)$ & \\
\hline \multirow{2}{*}{ Extension } & Superficial & 4 & 1.0 & 0.1 & 1.0 & 0.97 \\
\hline & Deep & 26 & $1.49(0.19,11.37)$ & & $0.98(0.28,3.45)$ & \\
\hline \multirow{3}{*}{ Radiation } & No & 10 & 1.0 & 0.14 & 1.0 & 0.07 \\
\hline & Primary & 2 & $1.78(0.32,9.78)$ & & $0.55(0.31,0.97)$ & \\
\hline & Adjuvant & 16 & $0.45(0.14,1.43)$ & & $0.34(0.11,1.02)$ & \\
\hline \multirow{3}{*}{ Chemotherapy } & No & 18 & 1.0 & 0.6 & 1.0 & 0.09 \\
\hline & Primary & 4 & $3.35(0.67,16.71)$ & & $0.48(0.21,1.1)$ & \\
\hline & Adjuvant & 6 & $1.24(0.33,4.64)$ & & $1.29(0.4,4.14)$ & \\
\hline \multirow{3}{*}{ AJCC } & I and II & 10 & 1.0 & 0.021 & 1.0 & 0.27 \\
\hline & III & 12 & $2.24(1.13,4.44)$ & & $2.59(0.74,8.98)$ & \\
\hline & IV & 8 & $6.29(1.2,33.06)$ & & $2.14(0.57,8.02)$ & \\
\hline \multirow{3}{*}{ MSKCC } & 0 and $I$ & 5 & 1.0 & 0.11 & 1.0 & 0.14 \\
\hline & II & 7 & $2.2(0.84,5.8)$ & & $1.79(0.82,3.88)$ & \\
\hline & III and IV & 18 & $2.25(0.29,17.76)$ & & $1.61(0.45,5.76)$ & \\
\hline \multirow{2}{*}{$\mathrm{N} 1$ or M1 } & No & 22 & 1.0 & 0.11 & 1.0 & 0.67 \\
\hline & Yes & 8 & $2.39(0.81,7.02)$ & & $1.24(0.47,3.3)$ & \\
\hline \multirow{2}{*}{ TNM } & T1aN0M0-T1bN1M1 & 12 & 1.0 & 0.18 & 1.0 & 0.07 \\
\hline & T2aN0M0-T2bN1M1 & 18 & $2.2(0.7,6.96)$ & & $2.9(0.93,9.08)$ & \\
\hline
\end{tabular}


Figure 1. (a) DFS and OS according to margin status. (b) DFS and OS according to size in Soft Tissue Sarcoma Cases.
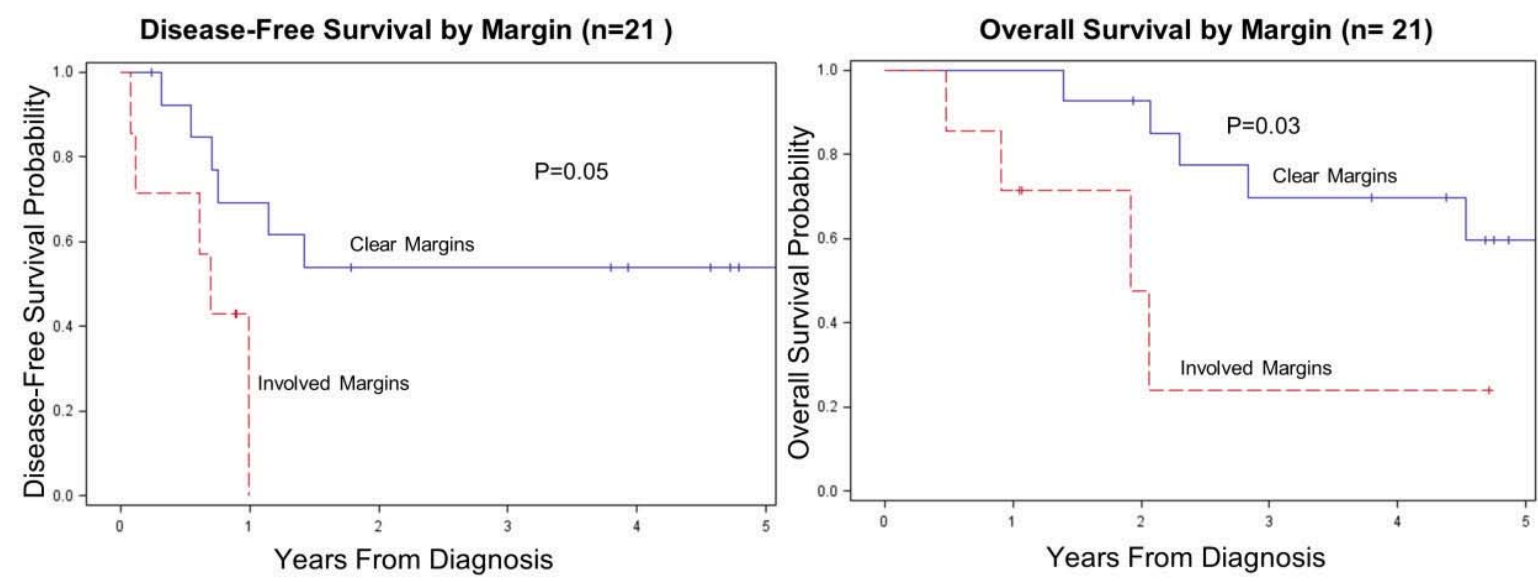

Disease-Free Survival by Size $(n=24)$
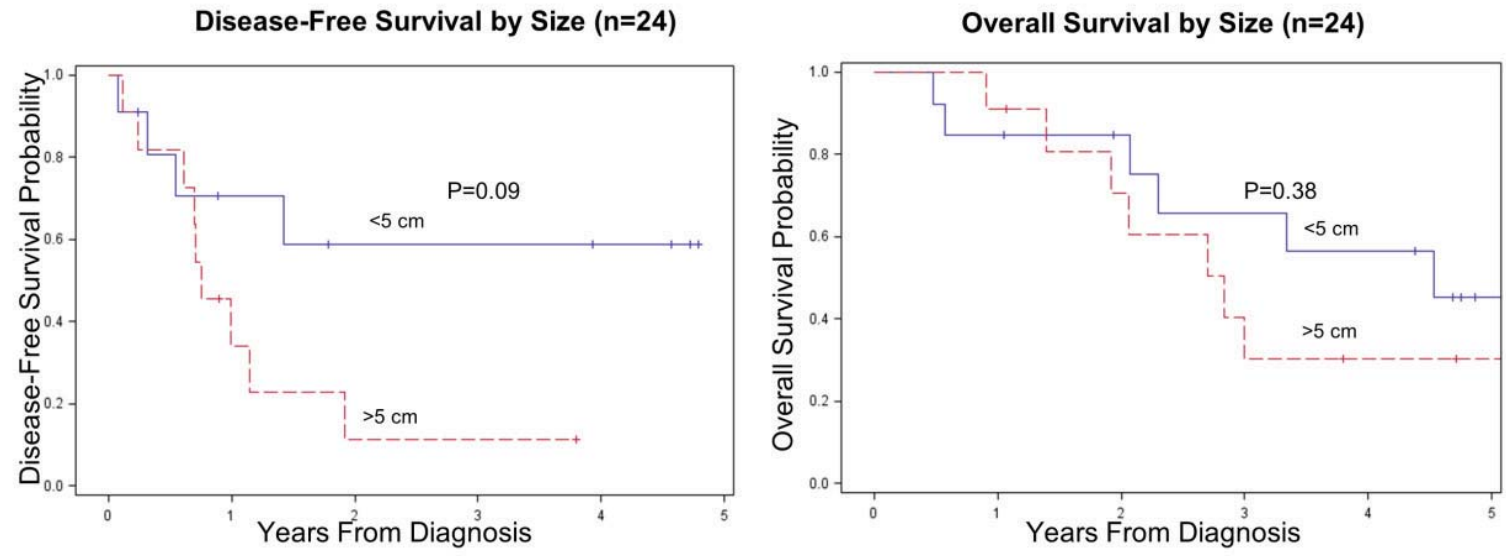

\section{Results for Osteogenic Sarcoma Cohort}

\subsection{Patient and Tumor Characteristics}

From 1999-2009, seven patients were treated for an osteosarcoma of the head and neck at Fox Chase Cancer Center. All were localized at diagnosis (AJCC Stage IA: four patients, IIA: two patients, IIB: one patient). Median age at diagnosis was much younger than that of soft tissue sarcoma (27.1 years, range 18.6-46.8) and all but one tumor was located in the mandible. One case was a chondroblastic osteosarcoma, while all others were osteosarcoma NOS.

\subsection{Treatment}

A total of $71 \%(5 / 7)$ patients received pre-operative systemic therapy generally consisting of cisplatin and an anthracycline $+/-$ high dose methotrexate. Two patients were not given pre-operative chemotherapy based on low grade and small size of their tumors. Although not customarily described in the pathologic report, it seems only one tumor (Stage IIA of the mandible) had an excellent response to induction chemotherapy (95\% necrosis). Forty-three percent (three of seven) of patients received adjuvant systemic therapy, generally with similar agents. Of the four 
patients who did not receive adjuvant chemotherapy, one had a low grade tumor, one patient refused and reasons for not administering adjuvant chemotherapy for the remaining two cases are unclear.

All patients with mandibular osteosarcomas underwent composite resection of the jaw and necessary soft tissue with reconstruction as a definitive surgical procedure. There were no elective neck dissections, but neck vessels were exposed in the course of free tissue transfer. One patient with a maxillary primary tumor was submitted to infrastructure maxillectomy, with subsequent fabrication of a custom obturator. Margins were clear in three patients, close $(\leq 1 \mathrm{~mm})$ in two patients and involved by cancer in two patients. All reconstructions were successful, although two patients had complications requiring additional operative procedures.

Involved and/or close margins were considered indications for adjuvant radiotherapy. Weekly low dose cisplatin $\left(30 \mathrm{mg} / \mathrm{m}^{2}\right)$ was administered with radiation. Both patients with involved margins and one of two patients with a c lose margin received adjuvant chemoradiation (median dose $6,600 \mathrm{cGy}$, range 6,000-6,600 cGy). There were no $\geq$ grade 3 radiation complications.

\subsection{Recurrence and Survival}

The median follow-up was 3.1 years (range 1.0-5.1 years). There were two recurrences, both of which were local recurrences within a year of completion of therapy. One recurrence was in a patient with high grade tumor and an involved margin who received adjuvant radiation and systemic chemotherapy. The other failure was of a low grade lesion with a clear margin who had received standard cisplatin and anthracycline chemotherapy (three cycles prior to surgery and two cycles after; the planned 6th cycle was not delivered secondary to disease recurrence). Both patients died of disease at 1.6 and 0.7 years from completion of therapy respectively. Crude locoregional control was therefore $71 \%$, and not affected by margin status (involved/close margin locoregional control: $75 \%$; clear margin locoregional control: $67 \%$ ).

\section{Discussion}

There have been few reports addressing the behavior and treatment of head and neck sarcomas in adults. This report represents an effort to describe outcomes and identify important prognostic factors among a contemporary cohort.

The 5-year disease-free and overall survival for our cohort of soft tissue sarcoma patients was $35 \%$ and $46 \%$, slightly lower than suggested in the literature [5-12], perhaps because a majority (23 of 30) of our patients had high grade tumors. Another difference we n oted was a higher percentage of synovial cell sarcomas compared with other series. This is unusual and may be due to our purely adult population. Synovial cell histology did not predict for patient outcome but analysis is limited due to the small number of cases. On univariate analysis, margin status and size were associated with survival, consistent with previous findings [6,13-18]. These factors seem related because it is more difficult to derive clear surgical margins with larger tumors - but our numbers do not permit multivariate analysis. This finding emphasizes that every thorough effort should be made to resect these tumors completely in the initial resection, including the use of modern reconstruction techniques. This is best accomplished at high-volume surgical centers with accomplished head and neck and reconstructive surgeons as well as experienced members of adjunctive services such as 
speech-language pathologists. Returning to high quality of life after en bloc resection about the head and neck region is demanding because of functional and cosmetic sequelae of the necessary resection. However, the extent and adequacy of excision may inform survival and incidence of local recurrence.

Unlike other series $[5,12,19-21]$, we did not find age or grade to be prognostic factors for disease control. This may be attributed to our sample size and larger number of patients with high grade tumors. Higher-grade sarcomas are more aggressive than low grade disease, recurring locally as well as metastasizing, thus leading to poorer outcomes.

Surgery remains the mainstay of treatment for head and neck sarcomas. Noteworthy exceptions to this principle include most rhabdomyosarcomas and Ewing's sarcomas. Radiation therapy is indicated after resection of all high grade sarcomas, large tumors, and when margins of resection are close or microscopically involved. There was no difference in local control or overall survival between patients in our series who were given postoperative radiotherapy and those who were not. This suggests that radiation is effective since the group that received adjuvant radiation had proportionally more aggressive tumors. Systemic chemotherapy is recommended for those tumors with a significant risk of distant metastases.

High local failure rates in the head a nd neck have historically been associated with poorer treatment outcomes in this group. Median DFS and OS in our series are worse compared to those achieved with other sub-sites [3,22-24], despite the finding that sarcomas arising in the head and neck have a lower probability of distant spread. This is commonly attributed to earlier diagnosis with a higher proportion of small tumors.

At disease relapse, repeat surgery and/or radiation should be considered to maximize control $[25,26]$. In addition, enrollment of these sarcoma patients to clinical trials investigating novel systemic therapies remains a priority.

Although limited by a small number of patients in our study, head and neck osteosarcoma appears to be a different disease than soft tissue sarcoma. It affects younger patients than does soft tissue sarcoma, is not as frequently of high grade, and appears to have a better prognosis. Most patients had lesions of the mandible, which along with the maxilla, is the most common site of primary tumors $[4,27]$. Due to small numbers, we were unable to determine prognostic factors (size, margin status or grade) predictive of recurrence. A large retrospective series from MD Anderson, however, indicated that high grade tumors, radiation induced tumors, and involved margins were associated with a poorer disease free survival and overall survival at five years [27]. Patients in that series with involved or uncertain margins who received chemotherapy had improved outcomes compared to those who were treated with surgery alone. They demonstrated no benefit from radiation alone, however only $23 \%$ of the patients were treated with radiation in the postoperative setting. Our institutional experience supports a p olicy of surgical extirpation with reconstruction, systemic therapy, and risk adapted use of external beam radiotherapy. None of our patients developed distant metastases, but there are reports with up to $21 \%$ of patients with distant progression (primarily to lung, bone, and brain) [27,28]. 


\section{Conclusions}

Patients with head and neck sarcomas should undergo wide excision with emphasis on removal of gross disease and attaining clear surgical margins. In most patients, except those with small, low grade lesions, postoperative radiation therapy should be added to maximize local control. Head and neck sarcomas are rare tumors that can present management difficulties. These tumors are best managed in a multi-disciplinary setting.

\section{Acknowledgments}

We would like to thank Saida M. Saleh for her support with statistical analysis.

\section{Conflicts of Interest}

The authors declare no conflict of interest.

\section{References}

1. Potter, B.O.; Sturgis, E.M. Sarcomas of the head and neck. Surg. Oncol. Clin. N. Am. 2003, 12,379 .

2. Hoffman, H.T.; Robinson, R.A.; Spiess, J.L.; Buatti, J. Update in management of head and neck sarcoma. Curr. Opin. Oncol. 2004, 16, 333.

3. Zagars, G.K.; Ballo, M.T.; Pisters, P.W.; Pollock, R.E.; Patel, S.R.; Benjamin, R.S.; Evans, H.L. Prognostic factors for patients with localized soft-tissue sarcoma treated with conservation surgery and radiation therapy: An analysis of 1,225 patients. Cancer 2003, 97, 2530.

4. Kassir, R.R.; Rassekh, C.H.; Kinsella, J.B.; Segas, J.; Carrau, R.L.; Hokanson, J.A. Osteosarcoma of the head and neck: Meta-analysis of nonrandomized studies. Laryngoscope 1997, 107, 56-61.

5. Willers, H.; Hug, E.B.; Spiro, I.J.; Efird, J.T.; Rosenberg, A.E.; Wang, C.C. Adult soft tissue sarcomas of the head and neck treated by radiation and surgery or radiation alone: Patterns of failure and prognostic factors. Int. J. Radiat. Oncol. Biol. Phys. 1995, 33, 585-593.

6. Kraus, D.H.; Dubner, S.; Harrison, L.B.; Strong, E.W.; Hajdu, S.I.; Kher, U.; Begg, C.; Brennan, M.F. Prognostic factors for recurrence and survival in head and neck soft tissue sarcomas. Cancer 1994, 74, 697-702.

7. Dijkstra, M.D.; Balm, A.J.; Coevorden, F.V.; Gregor, R.T.; Hart, A.A.; Hilgers, F.J.; Keus, R.B.; Loftus, B.M. Survival of adult patients with head and neck soft tissue sarcomas. Clin. Otolaryngol. 1996, 21, 66-71.

8. Barker, J.L.; Paulino, A.C.; Feeney, S.; McCulloch, T.; Hoffman, H. Locoregional treatment for adult soft tissue sarcomas of the head and neck: An institutional review. Cancer J. 2003, 9, 49-57.

9. Le Vay, J.; O’Sullivan, B.; Catton, C.; Cummings, B.; Fornasier, V.; Gullane, P.; Simm, J. An assessment of prognostic factors in soft tissue sarcoma of the head and neck. Arch. Otolaryngol. Head Neck Surg. 1994, 120, 981-986. 
10. Le, Q.T.; Fu, K.K.; Kroll, S.; Fitts, L.; Massullo, V.; Ferrell, L.; Kaplan, M.J., Phillips, T.L. Prognostic factors in adult soft-tissue sarcomas of the head and neck. Int. J. Radiat. Oncol. Biol. Phys. 1997, 37, 975-984.

11. Chen, S.A.; Morris, C.G.; Amdur, R.J.; Werning, J.W.; Villaret, D.B.; Mendenhall, W.M. Adult head and neck soft tissue sarcomas. Am. J. Clin. Oncol. 2005, 28, 259-263.

12. Penel, N.; Mallet, Y.M.; Robin, C.; Fournier, C.; Grosjean, J.; Ceugnart, L.; Clisant, S.; Lefebvre, J.L. Prognostic factors for adult sarcomas of head and neck. Int. J. Oral Maxillofac. Surg. 2008, 37, 428-432.

13. Farhood, A.I.; Hajdu, S.I.; Shiu, M.H.; Strong, E.W. Soft tissue sarcomas of the head and neck in adults. Am. J. Surg. 1990, 160, 365-369.

14. Tran, L.M.; Mark, R.; Meier, R.; Calcaterra, T.C.; Parker, R.G. Sarcomas of the head and neck: Prognostic factors and treatment strategies. Cancer 1992, 70, 169-177.

15. Weber, R.S.; Benjamin, R.S.; Peters, L.J.; Ro, J.Y.; Achon, O.; Goepfert, H. Soft tissue sarcomas of the head and neck in adolescence and adults. Am. J. Surg. 1986, 152, 286-292.

16. De Bree, R.; van der Valk, P.; Kuik, D.J.; van Diest, P.J.; Doornaert, P.; Buter, J.; Eerenstein, S.E.; Langendijk, J.A.; van der Waal, I.; Leemans, C.R. Prognostic factors in adult soft-tissue sarcomas of the head and neck: A single-centre experience. Oral Oncol. 2006, 42, 703-709.

17. Van Damme, J.P.; Schmitz, S.; Machiels, J.P.; Galent, C.; Gregoire, V.; Lengele, B.; Hamoir, M. Prognostic factors and assessment of staging systems for head and neck soft tissue sarcomas in adults. EJSO 2010, 36, 684-690.

18. Gonzalez-Gonzales, R.; Bologna-Molina, N.; Molina-Frechero, H.R.; Dominguez-Malagon, H.R. Prognostic factors and treatment strategies for adult head and neck soft tissue sarcoma. Int. J. Oral. Maxillofac. Surg. 2012, 41, 569-575.

19. Greager, J.A.; Patel, M.K.; Briele, H.A.; Walker, M.J.; Das Gupta, T.K. Soft tissue sarcomas of the head and neck. Cancer 1985, 56, 820-824.

20. Bentz, B.G.; Singh, B.; Woodruff, J.; Brennan, M.; Shah, J.P.; Kraus, D. Head and neck soft tissue sarcomas: A multivariate analysis of outcomes. Ann. Surg. Oncol. 2004, 11, 619-628.

21. Huber, G.F.; Matthews, T.W.; Dort, J.C. Soft tissue sarcomas of the head an d neck: A retrospective analysis of the Alberta experience 1974 to 1999. Laryngoscope 2006, 116, $780-785$.

22. Robinson, M.; Barr, L.; Fisher, C.; Fryatt, I.; Stotter, A.; Harmer, C.; Wiltshaw, E.; Westbury, G. Treatment of extremity soft tissue sarcomas with surgery and radiotherapy. Radiother. Oncol. 1990, 18, 221-223.

23. Sadoski, C.; Suit, H.D.; Rosenberg, A.; Mankin, H.; Efird, J. Preoperative radiation, surgical margins and local control of extremity sarcomas of soft tissues. J. Surg. Oncol. 1993, 52, 223-230.

24. Suit, H.D.; Mankin, H.J.; Wood, W.C.; Proppe, K.H. Preoperative, intraoperative and postoperative radiation in the treatment of primary soft tissue sarcoma. Cancer 1985, 55, 2659-2667.

25. Mendenhall, W.M.; Mendenhall, C.M.; Werning, J.W.; Riggs, C.E.; Mendenhall, N.P. Adult head and neck soft tissue sarcomas. Head Neck 2005, 27, 916. 
26. Dudhat, S.B.; Mistry, R.C.; Varughese, T.; Fakih, A.R.; Chinoy, R.F. Prognostic factors in head and neck soft tissue sarcomas. Cancer 2000, 89, 868.

27. Guadagnolo, B.A.; Zagars, G.K.; Raymond, A.K.; Benjamin, R.S.; Sturgis, E.M. Osteosarcoma of the jaw/craniofacial region outcomes after multimodality treatment. Cancer 2009, 115, 3262-3270.

28. Chennupati, S.K.; Norris, R.; Dunham, B.; Kazahaya, K. Osteosarcoma of the skull base: Case report and review of literature. Int. J. Ped. Otorhinolaryngol. 2008, 72, 115-119. 
Reprinted from Cancers. Cite as: Infante, T.; Cesario, E.; Gallo, M.; Fazioli, F.; De Chiara, A.; Tutucci, C.; Apice, G.; de Nigris, F. Ex Vivo Behaviour of Human Bone Tumor Endothelial Cells. Cancers 2013, 5, 404-417.

Article

\title{
Ex Vivo Behaviour of Human Bone Tumor Endothelial Cells
}

\section{Teresa Infante ${ }^{1}$, Elena Cesario ${ }^{2}$, Michele Gallo ${ }^{3}$, Flavio Fazioli ${ }^{3}$, Annarosaria De Chiara ${ }^{4}$,} Cristina Tutucci ${ }^{5}$, Gaetano Apice ${ }^{5}$ and Filomena de Nigris ${ }^{2} *$

1 SDN-Foundation, Institute of Diagnostic and Nuclear Development, IRCCS, 80143 Naples, Italy

2 Department of Biochemistry Biophysics and General Pathology, Second University of Naples, 80138 Naples, Italy

3 Division of Skeletal Muscles Oncology Surgery, National Cancer Institute, Pascale Foundation, 80131 Naples, Italy

4 Anatomic Pathology Unit, National Cancer Institute, Pascale Foundation, 80131 Naples, Italy

5 Medical Oncology of Bone and Soft Sarcoma tissues Unit, National Cancer Institute, Pascale Foundation, 80131 Naples, Italy

* Author to whom correspondence should be addressed; E-Mail: filomena.denigris@unina2.it; Tel.: +39-081-566-5686; Fax: +39-081-450-169.

Received: 19 February 2013; in revised form: 19 March 2013 / Accepted: 3 April 2013 /

Published: 11 April 2013

\begin{abstract}
Cooperation between endothelial cells and bone in bone remodelling is well established. In contrast, bone microvasculature supporting the growth of primary tumors and metastasis is poorly understood. Several antiangiogenic agents have recently been undergoing trials, although an extensive body of clinical data and experimental research have proved that angiogenic pathways differ in each tumor type and stage. Here, for the first time, we characterize at the molecular and functional level tumor endothelial cells from human bone sarcomas at different stages of disease and with different histotypes. We selected a CD $31^{+}$subpopulation from biopsies that displayed the capability to grow as adherent cell lines without vascular endothelial growth factor (VEGF). Our findings show the existence in human primary bone sarcomas of highly proliferative endothelial cells expressing CD31, CD44, CD105, CD146 and CD90 markers. These cells are committed to develop capillary-like structures and colony formation units, and to produce nitric oxide. We believe that a better understanding of tumor vasculature could be a valid tool for the design of an efficacious antiangiogenic therapy as adjuvant treatment of sarcomas.
\end{abstract}


Keywords: sarcomas; angiogenesis; endothelial cells; tumor microenvironment

\section{Introduction}

The unique characteristics of bone provide homing signals to different types of cancer cells, which together with biochemical (e.g., cytokines, growth factors) and physical (e.g., acidic pH, high extracellular calcium concentration) properties of the bone create an advantageous microenvironment for primary tumors and metastasis growth [1,2]. Efforts directed toward targeting tumor microenvironment for solid tumor therapy have largely focused on blocking blood vessels $[3,4]$. However, to date, the promising results reported in preclinical studies have not been reproduced in the clinical setting [5]. The lack of markers for monitoring antiangiogenic therapy and the fact that tumor endothelial cells (TECs) are different from normal endothelial cells (ECs) have contributed to short-lived response to treatment [6]. Identifying markers and overcoming the mechanisms that mediate resistance to antiangiogenic drugs will likely yield improved clinical outcomes. Several studies have shown that TECs differ from normal ECs in the expression of specific surface markers and genetic abnormalities responsible for incomplete, irregular and tortuous blood vessels with increased permeability $[1,2,7,8]$. These properties derive from tumor type, blood vessels site, and stage of tumor progression governed by extracellular environment including biochemical and signalling pathways as well as by cellular context [9].

The phenotypic and genetic heterogeneity of TECs raises the issue of whether they originate from normal ECs, bone marrow, resident stem cells, transdifferentiation or de-differentiation of tumor stem cells into ECs, or cell fusion between cancer cells and normal ECs $[1,10]$. Furthermore, the mechanism by which TECs acquire a co nstitutive proangiogenic phenotype and resistance to common antiangiogenic drugs is still unknown [11-13]. Bone sarcomas are a group of mesenchymal malignancies, highly vascularized with many different subtypes each with unique clinical pathological features [14]. To improve the outcome of unresectable, chemoresistant or metastatic patients, ongoing trials are testing antiangiogenic drugs as adjuvant therapy [15]. However, not all subtypes are expected to respond since bone tumor microenvironments are poorly understood and no data are available on the phenotypic and functional characteristics of TECs. Understanding the origin of bone TECs as well as their molecular and functional characteristics may help to develop appropriate and specific antiangiogenic therapies. Here, we characterize for the first time human bone TECs from fresh biopsies at functional and molecular levels.

\section{Experimental Section}

\subsection{Human Tissue Samples}

Tissues, surgically resected from 13 cases, were clinically classified as bone and soft sarcoma by histologic and immunohistologic criteria, according to UI CC/AJCC by Istituto Nazionale Tumori, Fondazione "G. Pascale" (Naples, Italy), under Local Ethical Committee approval. Specimens were finely minced with scissors and then digested by incubation for $1 \mathrm{~h}$ at $37^{\circ} \mathrm{C}$ in Dulbecco's modified Eagle's medium (DMEM) containing collagenase II (Sigma Chemical, Perth, 
WA, USA). After washings in medium plus 10\% FCS (GIBCO, Grand Island, NY, USA), cell suspension was forced through a graded series of meshes to s eparate the cell components from stromal cells and aggregates. Cells were pelleted, resuspended, and isolated using anti-CD31 Ab coupled to magnetic beads, with the MACS system (Miltenyi Biotec, Auburn, CA, USA). Briefly, $2 \times 10^{5}$ cells $/ \mu \mathrm{L}$ were labelled with the anti-CD31 mAb for $20 \mathrm{~min}$ and then were washed twice and resuspended in MACS buffer (PBS without $\mathrm{Ca}^{2+}$ and $\mathrm{Mg}^{2+}$, supplemented with $1 \%$ bovine serum albumin (BSA) and $5 \mathrm{mmol} / \mathrm{L}$ EDTA). After washings, cells were separated on a magnetic stainless steel wool column (Miltenyi Biotec.) according to the manufacturer's recommendations. CD31 ${ }^{+}$ cells were plated onto $0.1 \%$ gelatin and were grown in EGM-2 medium (GIBCO) supplemented with epidermal growth factor $(10 \mathrm{ng} / \mathrm{mL})$, hydrocortisone $(1 \mu \mathrm{g} / \mathrm{mL})$, and $0.5 \%$ FCS (GIBCO). Lack of contaminating leukocytes or of smooth muscle cells was verified by FACS analysis with anti-CD45 mAb and anti-smooth muscle actin Ab. Human aortic endothelial cells (HAEC) were obtained from ATCC, as described previously $[16,17]$.

\subsection{Cytofluorimetric Analysis}

Cells were detached from plates with non-enzymatic cell dissociation solution, washed in PBS containing $2 \%$ BSA to block remaining non-specific sites. $2 \times 10^{5}$ cells were then incubated for 30 min at $4{ }^{\circ} \mathrm{C}$ with the appropriate $\mathrm{Ab}$ or with the relevant control in PBS containing $2 \%$ BSA. For cytofluorimetric analysis the primary antibodies used were: anti CD31-FITC (R\&D Systems, Inc. Minneapolis, MN, USA), anti VE-cadherin-PE (Santa Cruz Biotechnology, Inc. Milan, Italy), anti CD133-PE (Miltenyi Biotech), anti fusin-PE (Santa Cruz Biotechnology), anti CD14-PE (R\&D Systems), anti CD44-FITC (R\&D Systems), anti CD13-PE/Cy5 (Chemicon International, Temecula, CA, USA), anti CD34-PE, anti CD105-PE, anti CD45-PE, anti CD90-PE, anti CD131-PE, anti CCR7-PE, anti CD133-PE (Miltenyi Biotech.), anti CD146-PE (Miltenyi Biotech.) and anti CD309-PE (Miltenyi Biotech.). Cells were analyzed on a FACS Vantage cell sorter (Becton Dickinson, Franklin Lakes, NJ, USA) and 30,000 event cells were analyzed at each experimental point. Control experiments included incubation with isotopic human IgG (Becton Dickinson). Data were analyzed using CellQuest software (Becton Dickinson).

\subsection{Immunofluorescence Studies}

For immunofluorescence $2 \times 10^{4}$ cells were grown on cover slides, fixed in $1 \%$ paraformaldehyde and first permeabilized with $0.1 \%$ Triton and then incubated with mouse anti-human CD31 (Chemicon International) or goat anti-human von-Willebrand (AbCam) at $4{ }^{\circ} \mathrm{C}$ for $45 \mathrm{~min}$. Cells were then stained by addition of FITC conjugated mouse anti-goat IgG or goat anti-mouse labelled with Texas red or Alexa Fluor 488 (Molecular Probe, Invitrogen, Milan, Italy), at 1:1,000 dilution. Cell nuclei were stained with DAPI (Sigma). Confocal microscopy analysis was performed using a Zeiss confocal microscope, (Carl Zeiss International, Berlin, Germany).

\subsection{Nitric Oxide and Platelet Activating Factor (PAF) Assays}

Nitric oxide (NO) production was detected using QuantiChrom TM Nitric Oxide Assay Kit (D2NO-100; Bioassay, San Diego, CA, USA). Cells were incubated for $60 \mathrm{~m}$ in at $37{ }^{\circ} \mathrm{C}$ in the 
working solution and then absorbance was read at $540 \mathrm{n} \mathrm{m}$. Cells were stimulated with acetylcholine in the absence or presence of the NO synthase inhibitor nitro-L-arginine methyl ester (L-NAME) (1 mM), and, as a co ntrol, with L-arginine (1 mM) (Bioassay). Three different experiments were performed at 5 th and 13th passages. Platelet Activating Factor (PAF) assay was performed using Platelet Activating Factor (PAF) ELISA Kit (Cayman Chemical, Ann Arbor, MI, USA) following the manufacturer's instructions.

\subsection{Cell Proliferation Assay}

$2 \times 10^{4}$ cells/well were seeded into 24 -well plates in EGM-2 containing FCS ranging between $0.5 \%-10 \%$ or plus growth factors (GIBCO). After 24,48 and $72 \mathrm{~h}$ of incubation under the appropriate conditions, monolayers were carefully washed, dried, and treated with $0.75 \%$ crystal violet in a solution of $50 \%$ ethanol, $0.25 \% \mathrm{NaCl}$, and $1.75 \%$ formaldehyde. After washing, the dye was eluted with $1 \%$ SDS in PBS, and absorbance was read at $595 \mathrm{~nm}$ with an ELISA reader (TECAN SPECTRA Fluor Plus fluorescence, absorbance, and luminescence reader, MTX Lab Systems, Inc., Vienna, VI, USA). Cell numbers were determined on the basis of a standard curve obtained with known cell numbers. All experiments were performed in triplicate. In addition, DNA synthesis was detected as incorporation of 5-bromo-2'-deoxyuridine (BrdU) into cellular DNA by using an ELISA kit (Roche Molecular Biochemicals, Basel, Switzerland), according to the manufacturer's instructions. Briefly, $10 \mu \mathrm{M}$ BrdU was added to the stimulated cells for $18 \mathrm{~h}$. Cells were then fixed with $0.5 \mathrm{M}$ ethanol- $\mathrm{HCl}$ and incubated with nuclease to digest the DNA. BrdU incorporated into the DNA was detected by using an anti-BrdU peroxidase-conjugated $\mathrm{mAb}$ and was visualized with a soluble chromogenic substrate. Optical density was measured with the ELISA reader at $450 \mathrm{~nm}$.

\subsection{Tube Formation and Migration Assay}

In vitro angiogenesis assay was performed on growth factor-reduced Matrigel diluted 1:1 with cold EGM-2 minimum media. HAECs or TECs $\left(1 \times 10^{4}\right.$ cells/well $)$ were seeded onto Matrigel-coated wells (12 multiwell plate) cultured with EGM-2 medium without growth factors containing $0.25 \%$ BSA. Additionally, migration assay was performed growing $1 \times 10^{4}$ cells in EGM-2 medium without growth factors in presence of $10 \mathrm{ng} / \mathrm{mL}$ SDF-1 (R\&D Systems). The capillary-like tubule structures in each well were photographed with Nikon light microscope, and experimental results were recorded. The mean of tube length $/ \mu \mathrm{m} \times$ field ( 3 different fields) was calculated with the MicroImage analysis system (Cast Imaging srl, Venice, Italy).

\subsection{Statistical Analysis}

Student t-test (two-tailed) was used for statistical evaluation. Level of significance was set at $p<0.05$. 


\section{Results}

\subsection{Characterization of TECs}

We examined 13 human bone sarcoma biopsies (with different clinical and histologic characteristics as indicated in Table 1) obtained after surgical resection at the Istituto Nazionale Tumori “G. Pascale", Naples (Italy).

Table 1. Clinical pathological features of bone biopsies.

\begin{tabular}{ccccc}
\hline Case & Gender & Age & Histotype & Grade \\
\hline 1 & M & 30 & GCT $*$ & I \\
2 & M & 25 & Chondrosarcoma & II \\
3 & M & 40 & Fibrosarcoma & I \\
4 & F & 35 & Osteosarcoma & II \\
5 & F & 45 & Chondrosarcoma & II \\
6 & F & 23 & Chondrosarcoma & II \\
7 & M & 43 & Osteosarcoma & III \\
8 & F & 31 & Fibrosarcoma & II \\
9 & M & 26 & Osteosarcoma & II \\
10 & M & 27 & GCT $*$ & I \\
11 & F & 22 & Chondrosarcoma & II \\
12 & F & 20 & GCT $*$ & I \\
13 & F & 45 & GCT * & I \\
\hline \multicolumn{5}{c}{ GCT: Giant cell tumor of bone. }
\end{tabular}

A subpopulation of $\mathrm{CD} 31^{+}$cells (a marker of endothelial cells) was selected from all biopsies by using magnetic beads coupled to CD31 an tibody. We chose CD31 an tibody because immunohistochemistry data on the same biopsies indicated that CD31 was expressed by vascular structures but not by tumor cells (Figure 1). After selection, TECs were established and grown without VEGF as adherent cells and were able to continue for several culture passages. Although at the first passage all cells lines were positive to CD31 antibody (Figures 2A,B), long-term culturing determined a reduction of CD31 (see below) and vWF positivity (Figure 2C).

Figure 1. Immunohistochemistry of bone sarcomas. (A) CD31-immunostained chondrosarcoma specimen (TEC 1 line); (B) ki67-stained chondrosarcoma specimen; (C) CD31-stained osteosarcoma specimen (TEC 2 line); (D) Ki67-stained osteosarcoma specimen.
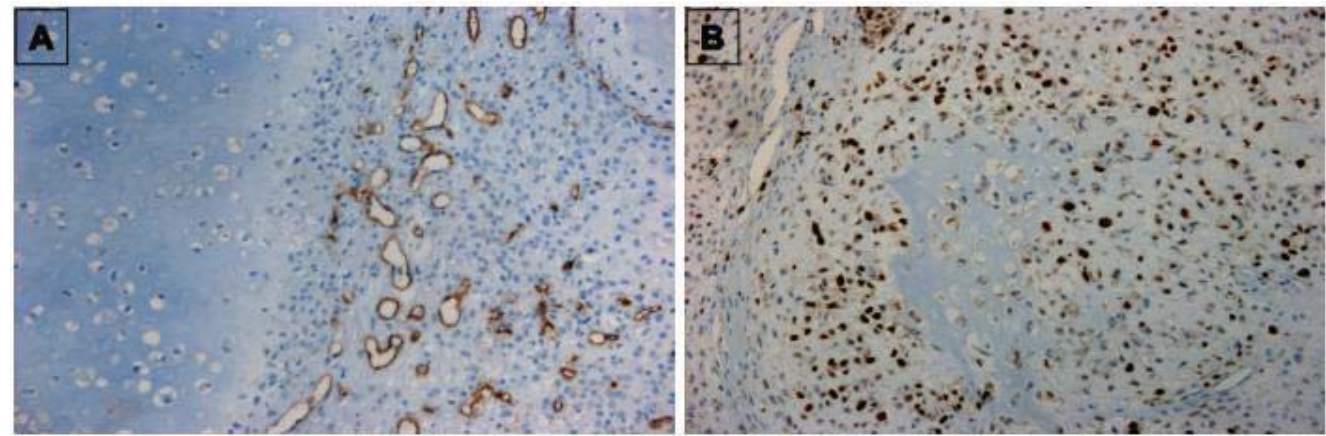
Figure 1. Cont.
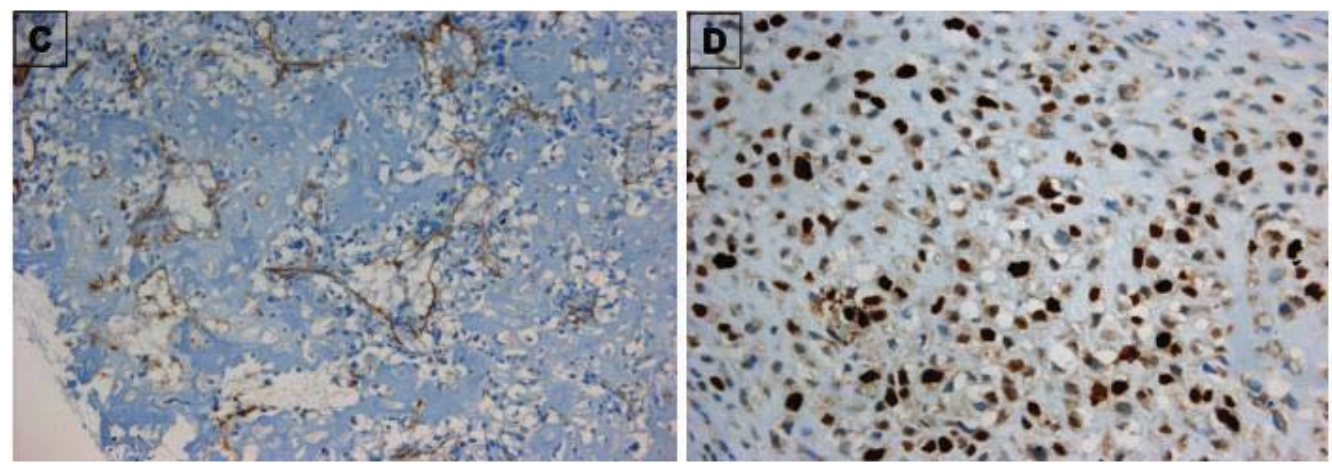

Figure 2. Cultured tumor endothelial cells from bone sarcoma. (A) Confluent tumor endothelial cells (TEC 1 line) stained with CD31, exhibiting the typical cobblestone structure (Panel A); (B) DAPI control staining; (C) Tumor endothelial cells TEC 1 stained with von Willebrand factor (cytoplasmatic staining).

CD31

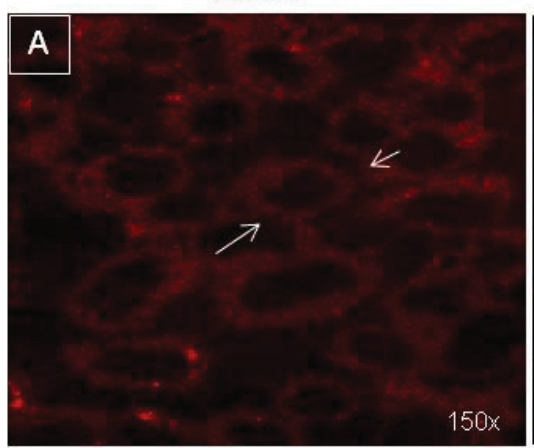

DAPI

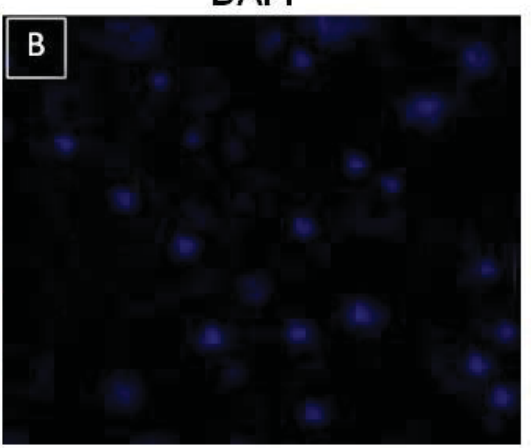

vWF

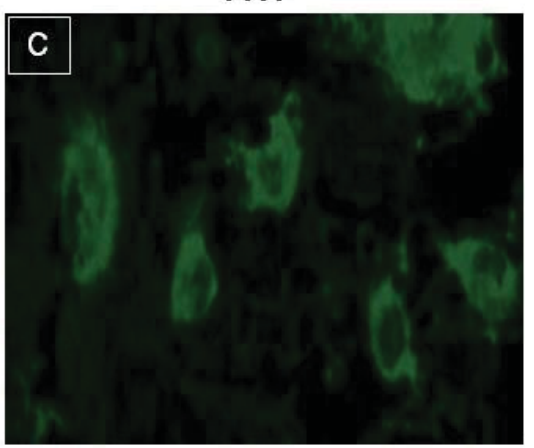

In addition, TECs showed a normal karyotype indicating they were not contaminated or originating from tumor cells (data not shown). TECs from different biopsies were subjected to cytofluorimetric analysis with endothelial markers and staminal markers, showing heterogeneous positivity. However, all TEC cell lines were strongly positive to CD105, CD90 and CD146 compared to normal ECs (Figure 3B) [18]. During culturing a lower expression of endothelial markers such as CD31, CD309 (VEGF receptor) and VE-Cadherin as well as mesenchymal markers such as CD117 and CD73 (ranging between 1\%-2\% of positive cells) was detected in TECs compared to the same cells at first passage (Figure 3B) consistent with cell growth in plate [19]. In contrast, 98\% of TECs expressed CD44 (compared to 30\% of HAECs), a marker of invasiveness possibly involved in cell motility. Concomitantly, TECs were negative to CD45, CD14 and smooth muscle actin Ab, suggesting non-contamination from bone-marrow cells, leukocytes and smooth muscle cells [20]. 
Figure 3. Characterization of TECs. (A) Expression of endothelial markers in a representative TEC line and HAEC cells, used as a control detected by FACS analysis. FACS analysis of HAECs, and TEC 1, with CD31 antibody at 1st passage and 3th passage. Expression of CD44 antigen in HAECs and TEC 1. (B) Bar graph expressing percentage of positive cells to specific antigens analyzed by FACS. Data are expressed as mean \pm SE of 10 different TEC lines grouped by histology and three control cell lines. Each experiment was repeated three times. TECs $v s$. HAECs $(p<0.001)$. IgG was used as negative control. (C) Representative example of TECs analyzed with CD146 antibody.

A
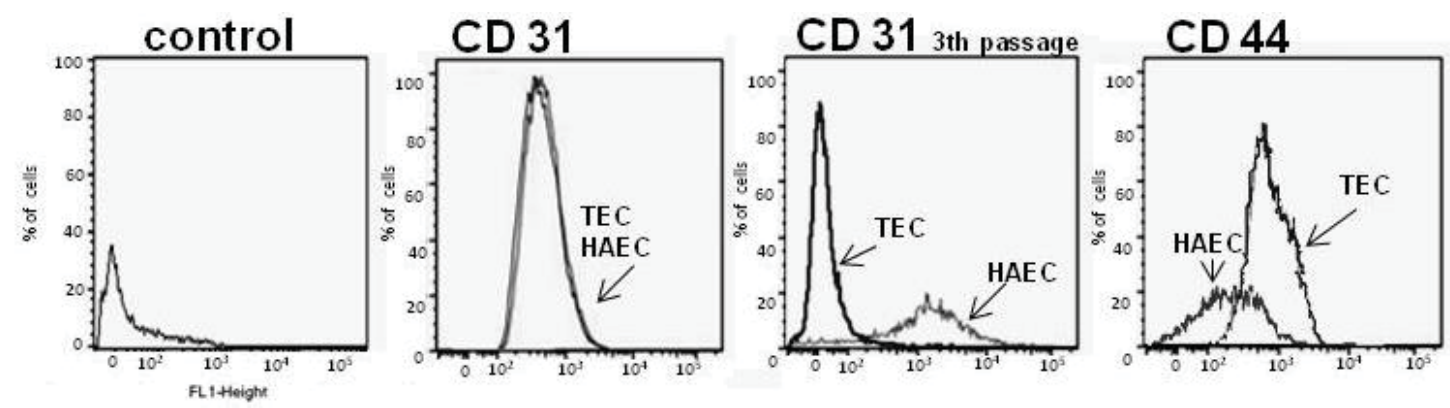

B
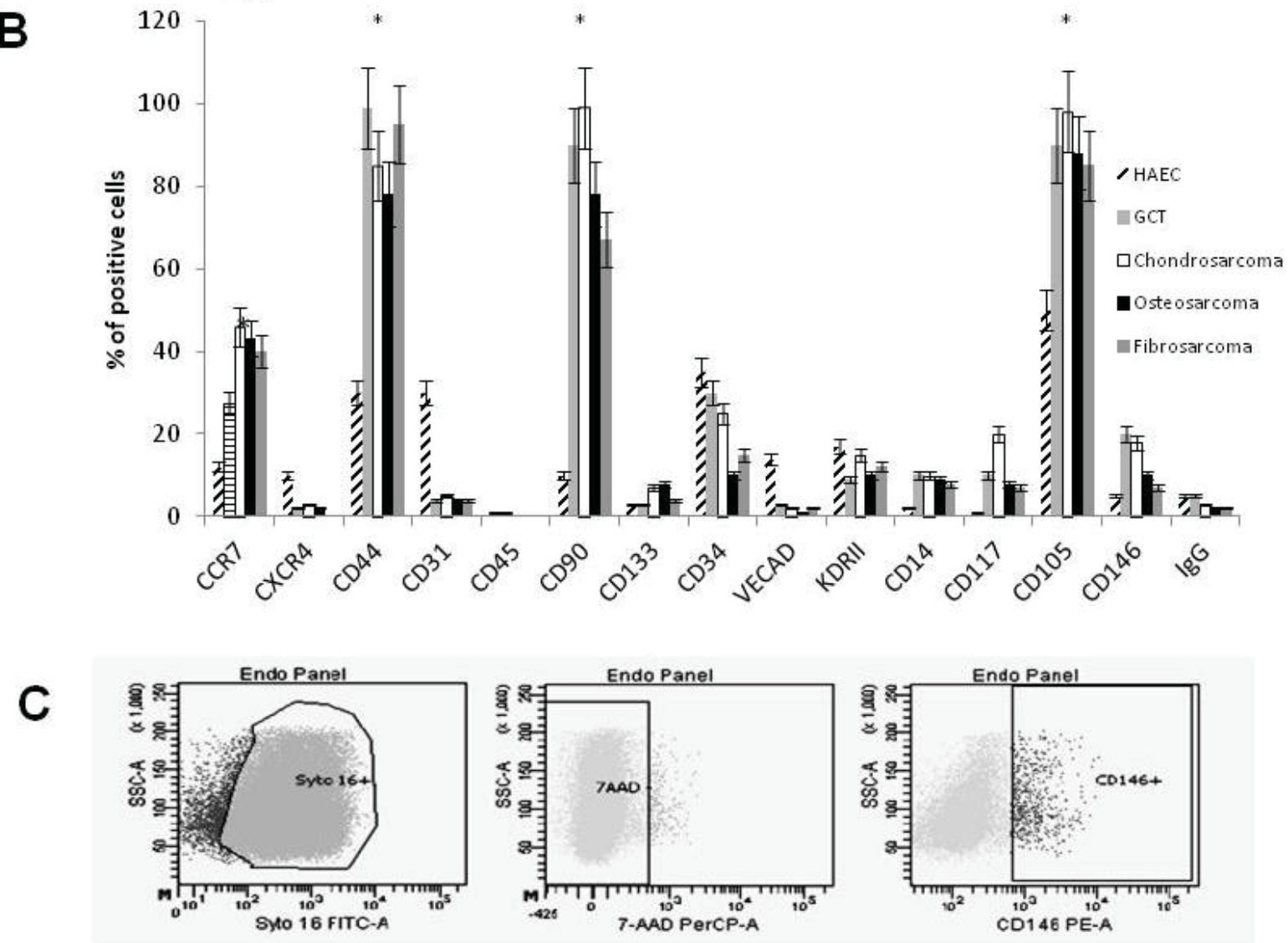

\subsection{In Vitro Behaviour of TECs}

To determine the behaviour of TECs in vitro, we selected two representative cell lines, TEC 1 and TEC 2 (from chondrosarcoma and osteosarcoma biopsies respectively and positive to CD44, CD105, CD90 and CD146), for further functional and molecular studies. Both cell lines showed proliferation at low serum concentration $(0.5 \%)$, and their growth rate was significantly higher than HAECs, even 
at the higher serum concentration and in the presence of serum plus growth factors known to represent optimal conditions for HAEC growth (Figure 4A,B).

Figure 4. Growth and functional characterization of TECs. (A) BrdU incorporation by TEC 1 (grey column), TEC 2 (black column) and HAECs (white column) cultured in EGM-2 in presence of $0.5 \%$ serum. (B) Proliferation of normal HAECs (white columns), TEC 1 (grey columns), and TEC 2 (black columns) in response to different concentrations of FCS or to FCS plus growth factors (GFs) added to medium for cell culture, evaluated after $72 \mathrm{~h}$. Results are the mean $\pm \mathrm{SE}$ of at least six experiments, $p<0.001 v s$. HAECs. (C) Nitric oxide assay. Cells were incubated with vehicle alone, $1 \mu \mathrm{M}$ acetylcholine, or $1 \mu \mathrm{M}$ acetylcholine in the presence of $10^{-3} \mathrm{M}$ of L-NAME. Activity of the endogenous NO system was controlled by stimulating cells with L-arginine, the substrate for NO synthase (data not shown). Experiments were repeated three times. NO values are expressed as cell absorbance at $540 \mathrm{~nm}$, mean \pm SE $p<0.001 v s$. control. (D) PAF production from $1 \times 10^{6}$ normal HAECs (white columns), TEC 1 (grey columns), and TEC 2 (black columns) incubated with vehicle or with $2 \mathrm{U} / \mathrm{mL}$ thrombin for $15 \mathrm{~min}$ at $37^{\circ} \mathrm{C}$. Data are the mean $\pm \mathrm{SE}$ of three individual experiments; $p<0.001 v s$. control (HAECs).

A

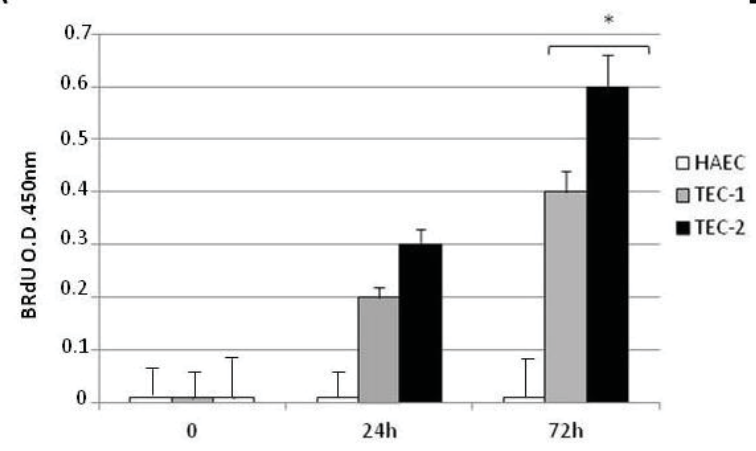

C

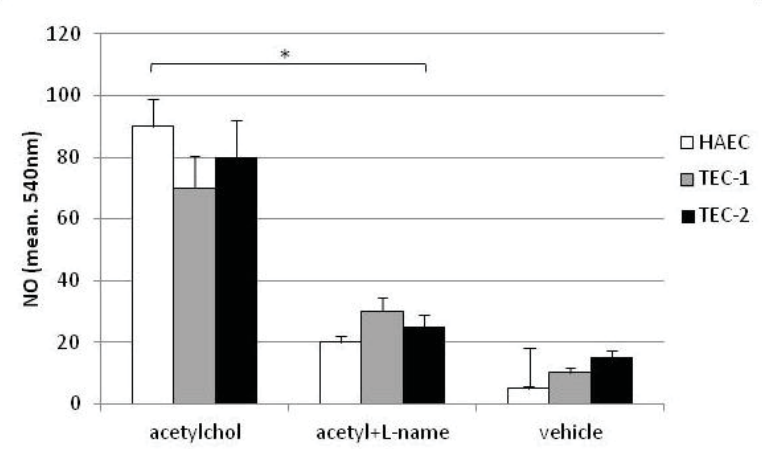

B

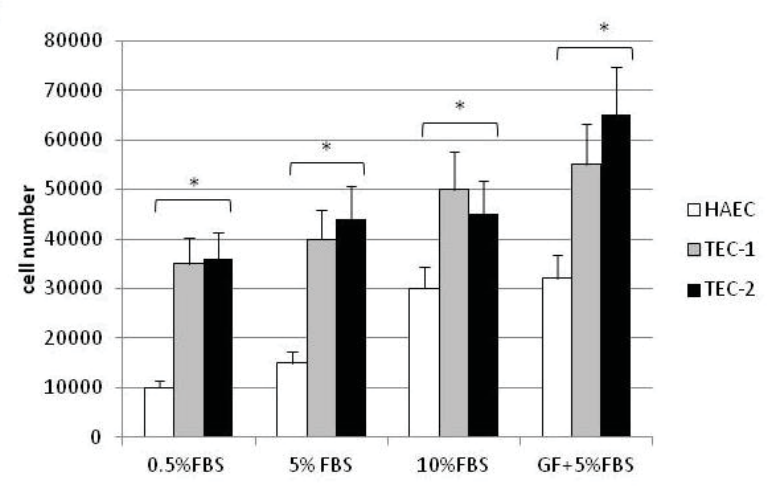

D

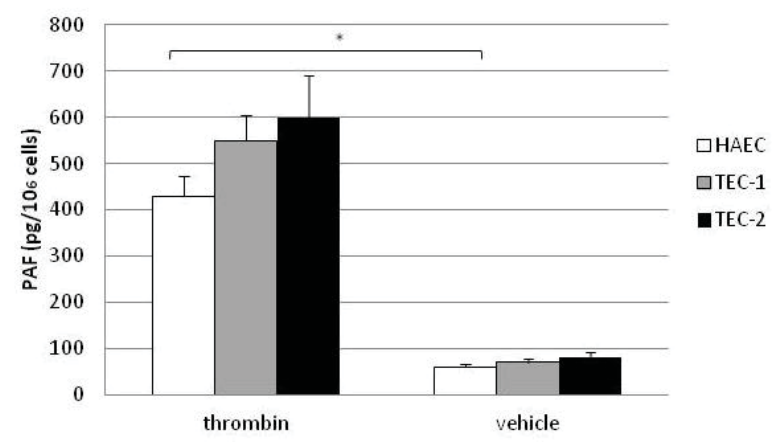

TEC 1 and TEC 2 were able to respond to acetylcholine and thrombin stimulation in terms of synthesis of NO and PAF, respectively (Figure 4C,D). To compare angiogenic properties of TECs and normal ECs we performed tube formation assays (Figure 5A). 
Figure 5. In vitro angiogenesis of TECs. Normal HAECs and TECs $\left(5.0 \times 10^{4}\right.$ cells/well $)$ were plated on growth factor-reduced Matrigel in EGM- 2 containing plus $0.2 \%$ BSA and were observed after 3 h. (A), (a) Representative micrograph of the weak network of tubes formed by HAECs in the absence of serum. (b) Representative micrograph of the complete network of tubes formed by TEC 2 cell line persisting for several days $(20 \times)$. (c) Bar graph of mean tube length $\mathrm{x}$ fields/ $\mu \mathrm{m}$ of different TEC cell lines (TEC 1 and TEC 2) evaluated by computer analysis system in 3 different fields at $20 \times$ magnification in duplicate wells of two different experimental points $(* p<0.05)$. (B, a,b) Typical examples of TEC colonies. As indicated by arrows an endothelial colony consists of a central core of rounded cells surrounded by sprouting cells. (C) Direct observation of spontaneous tube-forming process by endothelial bone tumor cells cultured with stromal-derived growth factor (SDF-1). (a-c) TEC 1 spontaneous tube formation in presence of SDF-1 stained with DAPI at different time points as indicated at $150 \times$ magnification; (a) $\mathrm{t}=0$; (b) $\mathrm{t}=30 \mathrm{~min} ;$ (c) $\mathrm{t}=1 \mathrm{~h}$; (d) HAEC cells in presence of $10 \mathrm{ng} / \mathrm{mL} \mathrm{SDF}-1 \mathrm{t}=1 \mathrm{~h}$. $(\mathbf{e}-\mathbf{g})$ direct in vitro micrograph of the tube formation at different time points; (e) $\mathrm{t}=0$; (f) $\mathrm{t}=30 \mathrm{~min} ;(\mathbf{g}) \mathrm{t}=1 \mathrm{~h}(20 \times$ magnification $)$.
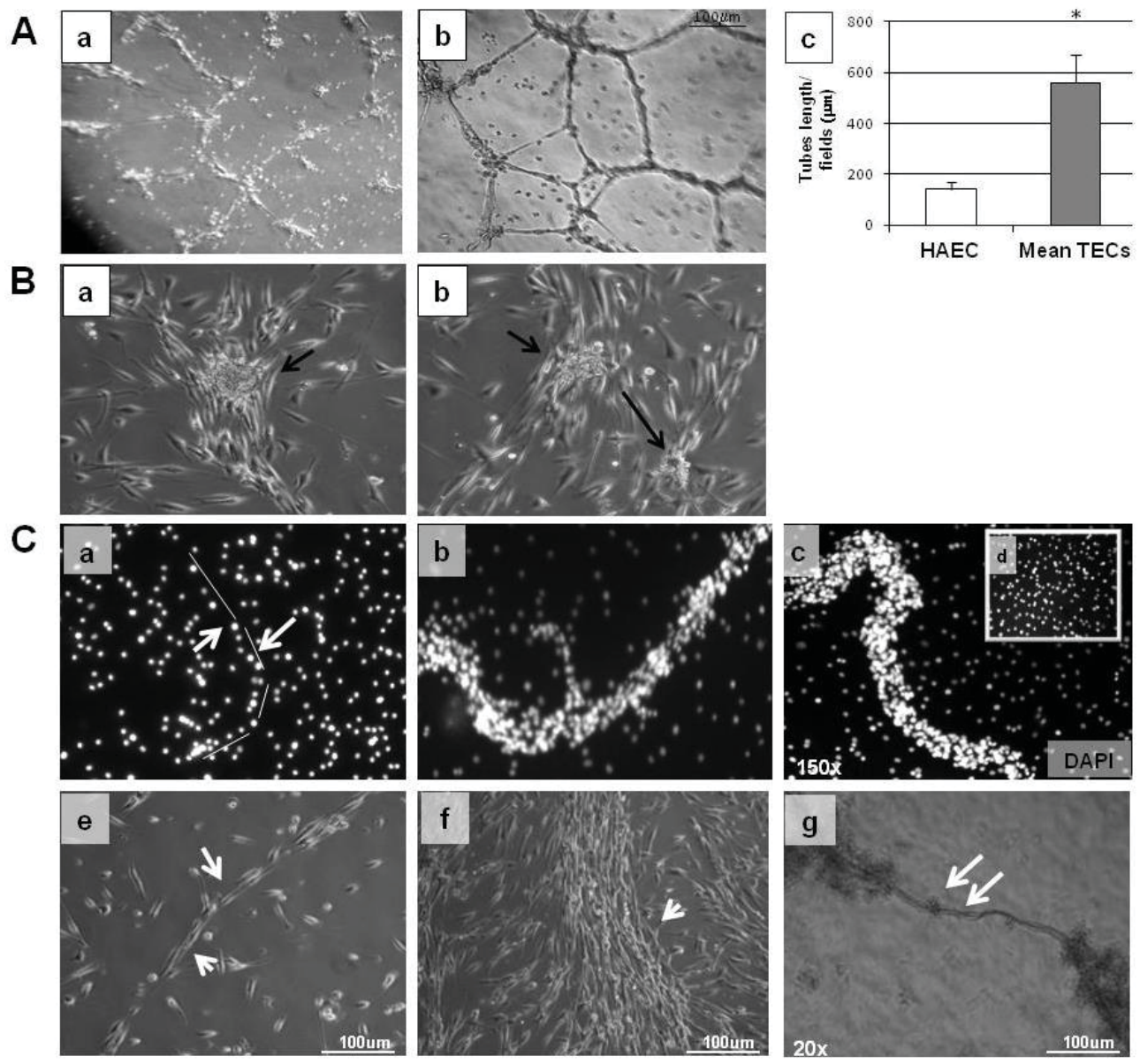

In contrast to HAECs, TECs showed the ability to make tubes when plated onto basal Matrigel in only a few minutes. TECs formed structures that persisted for several days in $0 \%$ serum (Figure $5 \mathrm{~A}$ panel b) unlike normal HAECs, which rapidly undergo apoptosis when plated onto Matrigel in the absence of serum (panel a). At $4 \mathrm{~h}$, under conditions providing optimal branch formation by 
HAECs ( $5 \%$ FCS, tube length $170 \pm 10 \mu \mathrm{m} \times$ field), TECs showed enhanced formation of branches $(570 \pm 20 \mu \mathrm{m}$ tube length $\times$ field; $* p<0.05)$. No differences in the spontaneous random motility of TECs were observed compared with normal HAECs (data not shown). In addition, TECs were able spontaneously to migrate one to another and organize themselves into colony formation units (Figure 5B), while in presence of stromal-derived growth factor (SDF-1) they were able to form spontaneous tubular structures (Figure 5C). Together, these data indicate that TECs not only present an endothelial phenotype but also behave as ECs.

\section{Discussion}

Here, we introduce for the first time the concept of bone tumor endothelial heterogeneity that arises from a co mplex heterotypic cross-talk interplay among osteoblasts, endothelium and environment $[21,22]$. In the present study, we isolated and characterized a population of endothelial bone tumor cells from 13 tumor biopsies with different grade and histology. TECs selected by CD31 antibody as already reported [23] lost this antigen early in vitro [24]. We selected from different histotypes an homogenous population expressing both endothelial and mesenchymal markers. Thus, TECs were positive, with different grade, to endothelial markers such as CD105, CD146, von Willebrand factor together with CD90, and, in addition, were strongly positive to CD44 antigen. Cells spontaneously showed an outgrowth forming colony units, normally associated with late endothelial progenitor cells (EPC) in other published studies [25,26]. Furthermore, recent observations have demonstrated that vascular endothelium itself contains highly proliferative endothelial colony-forming cell subpopulations (ECFCs), responsible for forming vessel lumens [27,28]. In contrast, no studies to date have determined whether these cells might represent the majority of angiogenesis response in tumors. Our functional studies demonstrated that bone $\mathrm{CD} 31^{+}$TECs are committed to forming vessels and, in contrast to normal ECs, and in presence of SDF-1, were able to differentiate into tubular structures in a few minutes. Although, these cells are a subpopulation of ECs present in the tumor, we observed that they are independent from tumor environment and VEGF to form vessels, consistent with previous reports [29,30], thus suggesting a permanent genetic change in their pro-angiogenic phenotype. The origin of TECs is still unknown; however, the presence of both endothelial and mesenchymal antigens indicates that they may originate from mesenchymal cells. EPCs and mesenchymal stem cells have the ability to trans-differentiate from one lineage to the other via an endothelial-to-mesenchymal process [31-33] however, it has been reported to have anticancer activity through the inhibition of angiogenesis $[34,35]$. The findings that TECs have stem cell features such as self renewal, migration capacity, longevity and normal karyotype but lack of main stem antigen such as CD133, suggest that TECs do not originate from tumor stem cells $[7,36]$. However, we cannot exclude that bone TECs derive from recruitment of EPCs following accumulation of genetic and epigenetic damage as reported by several studies [37]. We know that the present isolated TEC lines only partially represent bone TEC population because endothelium is heterogeneous in both tumor site and stage [36]. Further studies using different technical approaches and a better immunophenotyping will be needed to ch aracterize other endothelial cell populations in order to understand tumor vessel complexity [37,38]. Here, we hi ghlight for the first time that CD31 $1^{+}$bone TECs express a constitutive proangiogenic phenotype and have a different immunophenotype from normal 
endothelium, but have the same behaviour. We believe that TECs cultured from specific tumors are ideal tools to test genetic and chemical compounds in order to find angiogenic inhibitors on a high-throughput basis. In addition, cell-based assays using various TECs could be a rational approach to study ECs in tumor tissues. Finally, these findings may be of great importance in designing more tissue-specific antiangiogenic therapies for sarcoma and bone metastasis treatment or identifying novel markers to improve clinical outcomes $[15,39]$.

\section{Conclusions}

Endothelial cells were in the past considered genetically stable and therefore good candidates for specific therapies because they were not expected to develop resistance. Here, we show that tumor endothelial cells have a particular proangiogenic phenotype. We believe that with better knowledge of the molecular and functional characteristics of tumor endothelium, can become a valid device to design appropriate antiangiogenic therapeutic approaches and to define novel tools to improve the predictive value of imaging techniques.

\section{References}

1. Mundy, G.R. Metastasis to bone: Causes, consequences and therapeutic opportunities. Nat. Rev. Cancer 2002, 2, 584-593.

2. Guise, T. Examining the metastatic niche: Targeting the microenvironment. Semin. Oncol. 2010, 37, S2-S14.

3. Potente, M.; Gerhardt, H.; Carmeliet, P. Basic and therapeutic aspects of angiogenesis. Cell 2011, 146, 873-887.

4. De Nigris, F.; Schiano, C.; Infante, T.; Napoli, C. CXCR4 inhibitors: Tumor vasculature and therapeutic challenges. Recent Pat. Anticancer Drug Discov. 2012, 7, 251-264.

5. Hanahan, D.; Coussens, L.M. Accessories to the crime: Functions of cells recruited to the tumor microenvironment. Cancer Cell 2012, 21, 309-322.

6. Jain, R.K.; Duda, D.G.; Willett, C.G.; Sahani, D.V.; Zhu, A.X.; Loeffler, J.S.; Batchelor, T.T.; Sorensen, A.G. Biomarkers of response and resistance to an tiangiogenic therapy. Nat. Rev. Clin. Oncol. 2009, 6, 327-338.

7. St. Croix, B.; Rago, C.; Velculescu, V.; Traverso, G.; Romans, K.E.; Montgomery, E.; Lal, A.; Riggins, G.J.; Lengauer, C.; Vogelstein, B.; et al. Genes expressed in human tumor endothelium. Science 2000, 289, 1197-1202.

8. Bagley, R.G.; Honma, N.; Weber, W.; Boutin, P.; Rouleau, C.; Shankara, S.; Kataoka, S.; Ishida, I.; Roberts, B.L.; Teicher, B.A. Endosialin/TEM 1/CD248 is a per icyte marker of embryonic and tumor neovascularization. Microvasc. Res. 2008, 76, 180-188.

9. De Bock, K.; Cauwenberghs, S.; Carmeliet, P. Vessel abnormalization: Another hallmark of cancer? Molecular mechanisms and therapeutic implications. Curr. Opin. Genet. Dev. 2011, 21, 73-79.

10. Bussolati, B.; Deregibus, M.C.; Camussi, G. Characterization of molecular and functional alterations of tumor endothelial cells to design anti-angiogenic strategies. Curr. Vasc. Pharmacol. 2010, 8, 220-232. 
11. Di Tomaso, E.; Capen, D.; Haskell, A.; Hart, J.; Logie, J.; Jain, R.K.; McDonald, D.M.; Jones, R.; Munn, L.L. Mosaic tumor vessels: Cellular basis and ultrastructure of focal regions lacking endothelial cell markers. Cancer Res. 2005, 65, 5740-5749.

12. Virrey, J.J.; Golden, E.B.; Sivakumar, W.; Wang, W.; Pen, L.; Schönthal, A.H.; Hofman, F.M.; Chen, T.C. Glioma-associated endothelial cells are chemoresistant to temozolomide. J. Neurooncol. 2009, 95, 13-22.

13. Xiong, Y.Q.; Sun, H.C.; Zhang, W.; Zhu, X.D.; Zhuang, P.Y.; Zhang, J.B.; Wang, L.; Wu, W.Z.; Qin, L.X.; Tang, Z.Y. Human hepatocellular carcinoma tumor-derived endothelial cells manifest increased angiogenesis capability and drug resistance compared with normal endothelial cells. Clin. Cancer Res. 2009, 15, 4838-4346.

14. Bacci, G.; Longhi, A.; Versari, M.; Mercuri, M.; Briccoli, A.; Picci, P. Prognostic factors for osteosarcoma of the extremity treated with neoadjuvant chemotherapy 15 -year experience in 789 patients treated at a single institution. Cancer 2006, 106, 1154-1161.

15. Ganjoo, K.; Jacobs, C. Antiangiogenesis agents in the treatment of soft tissue sarcomas. Cancer 2010, 116, 1177-1183.

16. De Nigris, F.; Rossiello, R.; Schiano, C.; Arra, C.; Williams-Ignarro, S.; Barbieri, A.; Lanza, A.; Balestrieri, A.; Giuliano, M.T.; Ignarro, L.J.; et al. Deletion of Yin Yang 1 protein in osteosarcoma cells on cell invasion and CXCR4/angiogenesis and metastasis. Cancer Res. 2008, 68, 1797-1808.

17. De Nigris, F.; Crudele, V.; Giovane, A.; Casamassimi, A.; Giordano, A.; Garban, H.J.; Cacciatore, F.; Pentimalli, F.; Marquez-Garban, D.C.; Petrillo, A.; et al. CXCR4/YY1 inhibition impairs VEGF network and angiogenesis during malignancy. Proc. Natl. Acad. Sci. USA 2010, 107, 14484-14489.

18. Schiano, C.; Grimaldi, V.; Casamassimi, A.; Infante, T.; Esposito, A.; Giovane, A.; Napoli, C. Different expression of CD146 in human normal and osteosarcoma cell lines. Med. Oncol. 2012, 29, 2998-3002.

19. McDonald, D.M.; Foss, A.J. Endothelial cells of tumor vessels: Abnormal but not absent. Cancer Metastasis Rev. 2000, 19, 109-120.

20. Zöller, M. CD44: Can a cancer-initiating cell profit from an abundantly expressed molecule? Nat. Rev. Cancer 2011, 24, 254-267.

21. Fuchs, S.; Dohle, E.; Kolbe, M.; Kirkpatrick, C.J. Outgrowth endothelial cells: Sources, characteristics and potential applications in tissue engineering and regenerative medicine. Adv. Biochem. Eng. Biotechnol. 2010, 123, 201-217.

22. Kanczler, J.M.; Oreffo, R.O. Osteogenesis and angiogenesis: The potential for engineering bone. Eur. Cell Mater. 2008, 15, 100-114.

23. Bussolati, B.; Deambrosis, I.; Russo, S.; Deregibus, M.C.; Camussi, G. Altered angiogenesis and survival in human tumor-derived endothelial cells. FASEB J. 2003, 17, 1159-1161.

24. De Bock, K.; Cauwenberghs, S.; Carmeliet, P. Vessel abnormalization: Another hallmark of cancer? Molecular mechanisms and therapeutic implications. Curr. Opin. Genet. Dev. 2011, $21,73-79$.

25. Fadini, G.P.; Losordo, D.; Dimmeler, S. Critical reevaluation of endothelial progenitor cell phenotypes for therapeutic and diagnostic use. Circ. Res. 2012, 110, 624-637. 
26. Yoder, M.C.; Mead, L.E.; Prater, D.; Krier, T.R.; Mroueh, K.N.; Li, F.; Krasich, R.; Temm, C.J.; Prchal, J.T.; Ingra, D.A. Redefining endothelial progenitor cells via clonal analysis and hematopoietic stem/progenitor cell principals. Blood 2007, 109, 1801-1809.

27. Tilki, D.; Hohn, H.-P.; Ergün, B.; Rafii, S.; Ergün, S. Emerging biology of vascular wall progenitor cells in health and disease. Trends Mol. Med. 2009, 15, 501-509.

28. Zhu, H.; Shao, Q.; Sun, X.; Deng, Z.; Yuan, X.; Yu, D.; Zhou, X.; Ding, Y. The mobilization, recruitment and contribution of bone marrow-derived endothelial progenitor cells to the tumor neovascularization occur at an early stage and throughout the entire process of hepatocellular carcinoma growth. Oncol. Rep. 2012, 28, 1217-1224.

29. Huang, J.; Kontos, C.D. PTEN modulates vascular endothelial growth factor-mediated signaling and angiogenic effects. J. Biol. Chem. 2002, 277, 10760-10766.

30. Liu, W.; Ahmad, S.A.; Reinmuth, N.; Shaheen, R.M.; Jung, Y.D.; Fan, F.; Ellis, L.M. Endothelial cell survival and apoptosis in the tumor vasculature. Apoptosis 2000, 5, 323-328.

31. Díez, M.; Musri, M.M.; Ferrer, E.; Barberà, J.A.; Peinado, V.I. Endothelial progenitor cells undergo an endothelial-to-mesenchymal transition- like process mediated by TGFbetaRI. Cardiovasc. Res. 2010, 88, 502-511.

32. Kurose, K.; Hoshaw-Woodard, S.; Adeyinka, A.; Lemeshow, S.; Watson, P.H.; Eng, C. Genetic model of multi-step breast carcinogenesis involving the epithelium and stroma: Clues to tumour-microenvironment interactions. Hum. Mol. Genet. 2001, 10, 1907-1913.

33. Akino, T.; Hida, K.; Hida, Y.; Tsuchiya, K.; Freedman, D.; Muraki, C.; Ohga, N.; Matsuda, K.; Akiyama, K.; Harabayashi, T.; et al. Cytogenetic abnormalities of tumor-associated endothelial cells in human malignant tumors. Am. J. Pathol. 2009, 175, 2657-2667.

34. Secchiero, P.; Zorzet, S.; Tripodo, C.; Corallini, F.; Melloni, E.; Caruso, L.; Bosco, R.; Ingrao, S.; Zavan, B.; Zauli, G. Human bone marrow mesenchymal stem cells display anti-cancer activity in SCID mice bearing disseminated non-Hodgkin's lymphoma xenografts. PLoS One 2010, 1, e11140.

35. Ho, I.A.; Toh, H.C.; Ng, W.H.; Teo, Y.L.; Guo, C.M.; Hui, K.M.; Lam, P.Y. Human bone marrow-derived mesenchymal stem cells suppress human glioma growth through inhibition of angiogenesis. Stem Cells 2013, 31, 146-155.

36. Regan, E.R.; Aird, W.C. Dynamical systems approach to endothelial heterogeneity. Circ. Res. 2012, 111, 110-130.

37. Resch, T.; Pircher, A.; Kähler, C.M.; Pratschke, J.; Hilbe, W. Endothelial progenitor cells: Current issues on characterization and challenging clinical applications. Stem Cell Rev. 2012, 8, 926-939.

38. Langley, R.R.; Fidler, I.J. The seed and soil hypothesis revisited-The role of tumor stroma interactions in metastasis to different organs. Int. J. Cancer 2011, 128, 2527-2535.

39. Amini, A.R.; Laurencin, C.T.; Nukavarapu, S.P. Differential analysis of peripheral blood- and bone marrow-derived endothelial progenitor cells for enhanced vascularization in bone tissue engineering. J. Orthop. Res. 2012, 30, 1507-1515. 
Reprinted from Cancers. Cite as: G oudarzi, A.; Gokgoz, N.; Gill, M.; Pinnaduwage, D.; Merico, D.; Wunder, J.S.; Andrulis, I.L. Protein Kinase C Epsilon and Genetic Networks in Osteosarcoma Metastasis. Cancers 2013, 5, 372-403.

Article

\title{
Protein Kinase C Epsilon and Genetic Networks in Osteosarcoma Metastasis
}

\author{
Atta Goudarzi ${ }^{1,2, *}$, Nalan Gokgoz ${ }^{2}$, Mona Gill ${ }^{2}$, Dushanthi Pinnaduwage ${ }^{2}$, Daniele Merico ${ }^{3}$, \\ Jay S. Wunder ${ }^{2}$ and Irene L. Andrulis ${ }^{1,2}$ \\ 1 Department of Molecular Genetics, University of Toronto, 1 King's College Circle, Toronto, \\ ON M5S 1A8, Canada; E-Mail: andrulis@lunenfeld.ca \\ 2 Samuel Lunenfeld Research Institute, Mount Sinai Hospital, 600 University Ave., Toronto, \\ ON M5G 1X5, Canada; E-Mails: nalan@lunenfeld.ca (N.G.); monagill_@hotmail.com (M.G.); \\ pinnad@lunenfeld.ca (D.P.); wunder@lunenfeld.ca (J.S.W.) \\ 3 The Centre for Applied Genomics, The Hospital for Sick Children, MaRS Centre-East Tower, \\ 101 College Street Rm.14-701, Toronto, ON M5G 1L7, Canada; E-Mail: \\ daniele.merico@gmail.com \\ * Author to whom correspondence should be addressed; E-Mail: atta.goudarzi@utoronto.ca; \\ Tel.: +1-416-596-4200 (ext. 8255); Fax: +1-416-586-8663.
}

Received: 16 February 2013; in revised form: 22 March 2013 / Accepted: 26 March 2013 / Published: 8 April 2013

\begin{abstract}
Osteosarcoma (OS) is the most common primary malignant tumor of the bone, and pulmonary metastasis is the most frequent cause of OS mortality. The aim of this study was to di scover and characterize genetic networks differentially expressed in metastatic OS. Expression profiling of OS tumors, and subsequent supervised network analysis, was performed to discover genetic networks differentially activated or organized in metastatic OS compared to localized OS. Broad trends among the profiles of metastatic tumors include aberrant activity of intracellular organization and translation networks, as well as disorganization of metabolic networks. The differentially activated PRKC $\varepsilon$-RASGRP3-GNB2 network, which interacts with the disorganized DLG2 hub, was also found to $b$ e differentially expressed among OS cell lines with differing metastatic capacity in xenograft models. PRKCE transcript was more abundant in some metastatic OS tumors; however the difference was not significant overall. In functional studies, PRKCE was not found to be involved in migration of M132 OS cells, but its protein expression was induced in M112 OS cells following IGF-1 stimulation.
\end{abstract}


Keywords: osteosarcoma; metastasis; expression profiling; network analysis; Dynemo; protein kinase $\mathrm{C}$ epsilon

\section{Introduction}

Osteosarcoma (OS) is the most common primary malignant cancer of the bone, with nearly 1,000 diagnoses annually in North America [1,2]. High-grade intramedullary osteosarcoma ("conventional") constitutes the majority of cases, and is an aggressive disease that typically metastasizes to the lungs [1,2]. Approximately $10 \%-20 \%$ of patients present to the clinic with discernable metastasis, and a further 20\%-30\% develop metastasis despite aggressive treatment [3]. Currently neoadjuvant chemotherapy in combination with surgical resection of osteosarcomas can achieve 5-year overall survival rates of approximately 65\% [4,5]. However, delineation of the molecular mechanisms contributing to osteosarcoma metastasis has the possibility of identifying specific therapeutic targets that improve prognosis of patients with metastatic osteosarcoma.

Expression profiling is an "omic"-level discovery technique that allows the quantification of thousands of different transcripts simultaneously between different disease states [6]. This technology has many applications in the cancer field including distinguishing subtypes of a particular cancer, identifying transcripts differentially expressed between those types, and predicting the subtype of a cancer based on the expression of identified transcripts [6,7]. In recent years it has been shown that analysis of expression profiles at the level of sets of transcripts rather than individual transcripts has more statistical power, is more reproducible between studies, and provides informative context to the results [7-9]. There are many algorithms for gene-set or network-based analytical algorithms, including the popular gene-set enrichment analysis (GSEA) and the proprietary ingenuity pathway analysis (IPA) [10]. Additionally dozens of other network-based analytical algorithms have been published that either utilize different statistical methods or that interrogate a unique cellular application (reviews: [8,9]). Two such algorithms are the methods of Chuang et al. that aims to discover differentially "activated" genetic networks [11], and "Dynemo" published by Taylor et al. that aims to discover differentially organized genetic networks [12]. These algorithms have successfully been applied to uncover molecular alterations in expression profiles of poor-outcome breast cancers, and have achieved over $70 \%$ predictive accuracy in -classifying those tumor samples utilizing a five-fold cross-validation strategy $[11,12]$.

The aim of the present study was to identify differentially activated and organized networks in expression profiles of metastatic-at-diagnosis osteosarcomas (MD-OS) compared to ex pression profiles of localized-at-diagnosis osteosarcomas (LD-OS). This analysis would assist in the prioritization of candidate networks for in vitro characterization in osteosarcoma cell lines, including cells of differing metastatic capability. 


\section{Results}

\subsection{Unsupervised Hierarchical Clustering of Expression Profiles Reveals Distinct Subtypes of Osteosarcomas}

Expression profiling was performed on 46 LD-OS tumor samples and 17 MD-OS tumor samples using UHN "human $19 \mathrm{k}$ cDNA microarrays (H19K)", that contain approximately 19,000 cDNA spots mapped to approximately 9,000 unique transcripts. Unsupervised gene discovery techniques were used to identify a subset of 596 genes that exhibited at-least six-fold change in expression in at-least four tumors $(p \leq 0.001)$, and hierarchical clustering of tumors according to the expression pattern of these genes demonstrated two broad groups of tumors. One group (Figure 1B, red dendrogram) contained all but two of the MD-OS tumors, and the other group (Figure 1B, green and blue dendrograms) contained all but one of the LD-OS tumors. This analysis supports the notion that osteosarcomas of varying metastatic status at the time of diagnosis exhibit distinct expression patterns, and that division of tumors into these categories (localized at diagnosis or metastatic at diagnosis) is appropriate for subsequent supervised analysis.

Figure 1. (A) Unsupervised hierarchical clustering of 596 hi ghly variable genes. (B) Dendrogram depicting separation of MD-OS tumors (green bars) and LD-OS tumors based on expression of highly variable genes. (A) Unsupervised hierarchical clustering of OS tumor samples (Partek Genomics Suite) was performed on a subset of 596 genes found to be significantly differentially expressed within the dataset (expression varies by at least 6 fold, in at least 4 pa tients, $p \leq 0.001$ ), according to methods previously described $[13,14]$. (B) The dendrogram reveals two clusters, one with red dendrogram lines that contains mostly metastatic-at-diagnosis tumors (green rectangles). A second cluster, which can be subdivided further (blue and green dendrogram lines) contains mostly localized-at-diagnosis tumors (purple rectangles).
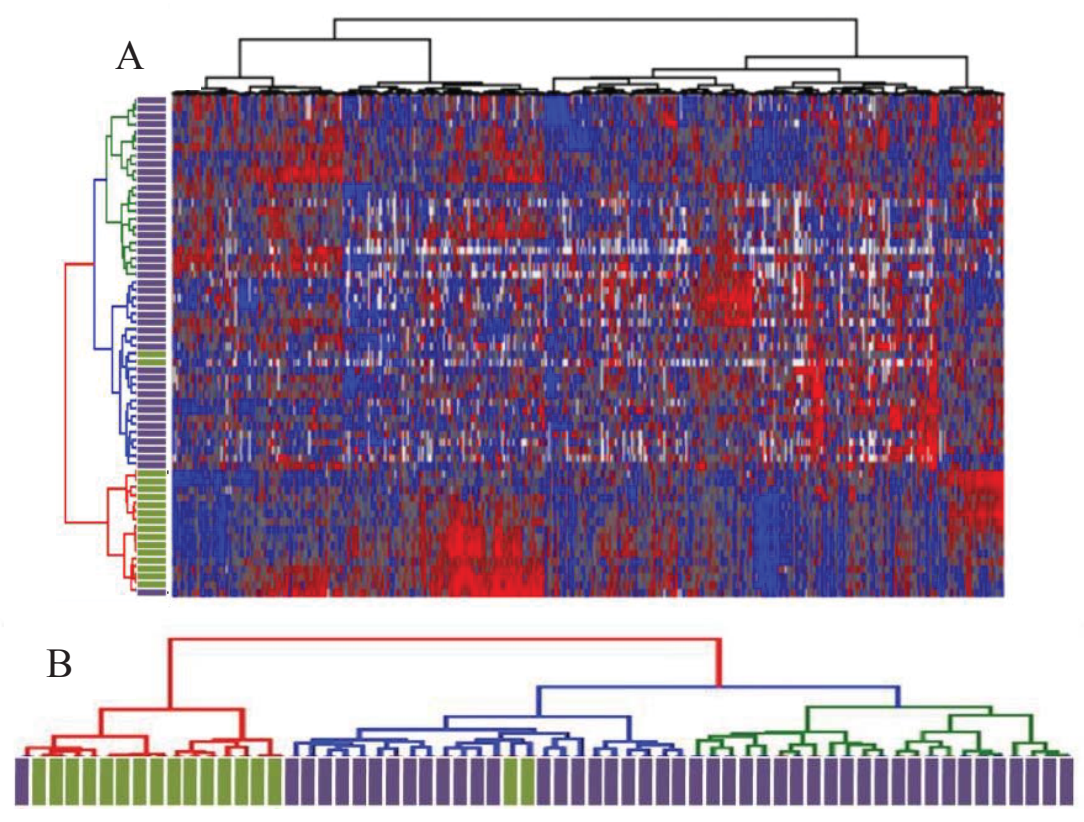


\subsection{Differentially Activated and Organized Networks in Metastatic Osteosarcomas}

The expression information was integrated with the Pathway Commons database of physical and genetic interactions [15], yielding a dat aset of 5,855 genetic networks. Supervised analysis was performed on these data in a manner similar to previous publications to identify the differentially activated and organized networks in MD-OS expression profiles. To describe broad trends in human metastatic OS, inclusive cut-off levels were identified to delineate as many networks as possible that were significantly differentially activated or organized in the metastatic samples. For this analysis, four hundred and ninety seven (497) of the 5,855 genetic networks were identified as differentially activated and six hundred and eighty three (683) networks were significantly differentially organized. Networks annotated to transport, translation, organization and protein modification were more commonly differentially activated than expected by chance (Table 1, column 8). Networks annotated to the processes of organization, transport and translation were more commonly differentially organized than expected by chance, as were metabolic networks (Table 1, column 11). Three hundred thirty eight (338) differentially activated, and one hundred and sixty two (162) differentially organized, networks were visualized and clustered according to their Gene Ontology Process annotation (Figure 2). This visualization allowed the observation that clusters of differentially activated networks interact with large, significantly disorganized hubs (e.g., Figure 2-"protein modification" process: cluster of differentially activated networks interacting with the disorganized hub breakpoint cluster region (BCR). This pattern is also observed in the "translation" and "transport" processes.

Figure 2. Nodes represent differentially activated (red squares) and organized (blue squares) networks in metastatic OS s amples. Green edges represent genetic overlap between networks. Genes in significant networks were annotated with simplified Gene Ontology Slim Generic terms (Table 6), and networks were grouped into processes by the most commonly occurring term in each network. Networks meeting the cut-off conditions detailed at the top of the figure were visualized with the Enrichment Map plugin for Cytoscape. The size of each node reflects the number of genes included in the network.

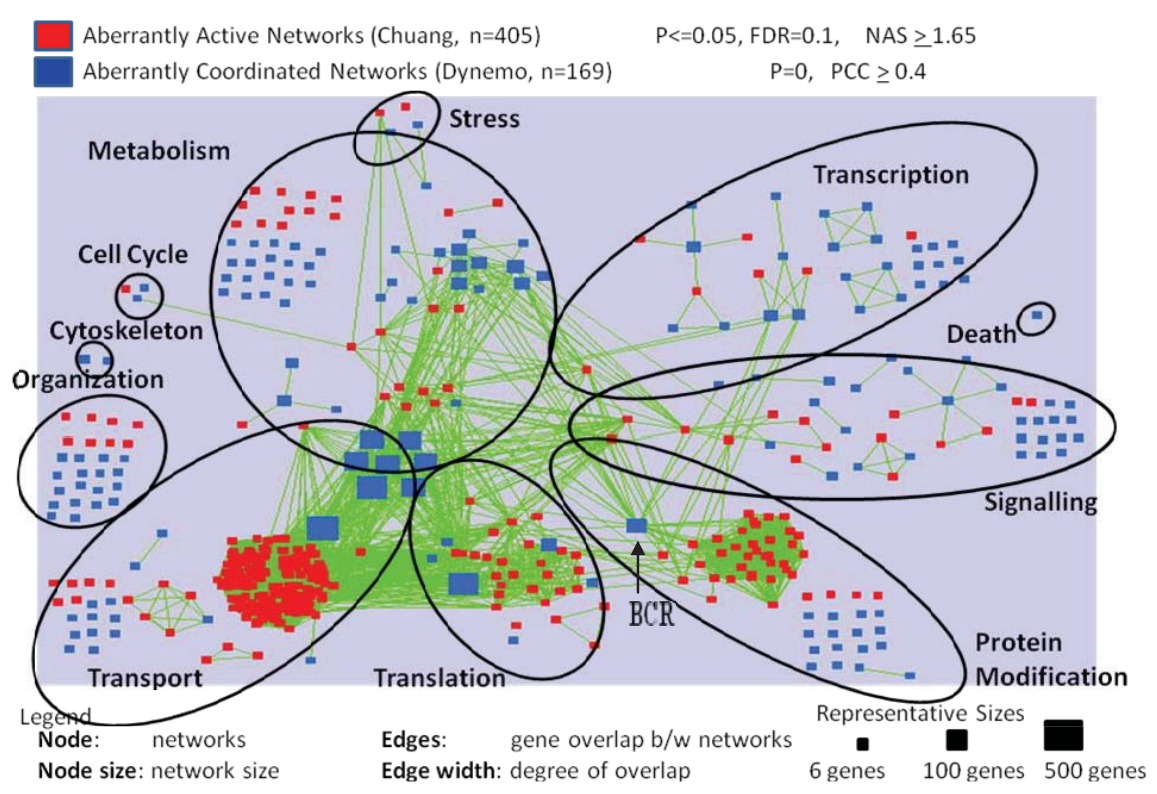


Table 1. Enrichment of genetic networks in metastatic osteosarcomas according to cellular processes at the global (Permissive) significance level.

\begin{tabular}{|c|c|c|c|c|c|c|c|c|c|c|}
\hline \multirow{3}{*}{$\begin{array}{c}\text { Cellular Processes } \\
1 \\
\end{array}$} & \multicolumn{4}{|c|}{ All Networks in Study } & \multicolumn{6}{|c|}{ Significant Networks: Permissive Cutoffs } \\
\hline & \multicolumn{2}{|c|}{ Subsets } & \multicolumn{2}{|c|}{ Entire Networks } & \multicolumn{3}{|c|}{ Differentially Activated } & \multicolumn{3}{|c|}{ Differentially Organized } \\
\hline & 2 & 3 & 4 & 5 & 6 & 7 & 8 & 9 & 10 & 11 \\
\hline & $\#$ & $\%$ of all & \# & $\%$ of all & $\#$ & $\%$ of all & $p$-value & $\#$ & $\%$ of all & $p$-value \\
\hline protein modification & 573 & 9.8 & 575 & 9.8 & 52 & $10.5 *$ & $5.3 \mathrm{E}-02$ & 49 & 7.2 & \\
\hline transport & 1002 & 17.1 & 869 & 14.8 & 194 & $39 *$ & $1.1 \mathrm{E}-34^{\dagger}$ & 133 & $19.5 *$ & $9.0 \mathrm{E}-05^{\dagger}$ \\
\hline signaling & 954 & 16.3 & 955 & 16.3 & 69 & 13.9 & & 87 & 12.7 & \\
\hline transcription & 595 & 10.2 & 635 & 10.8 & 17 & 3.4 & & 48 & 7 & \\
\hline stress & 247 & 4.2 & 257 & 4.4 & 15 & 3 & & 9 & 1.3 & \\
\hline metabolism & 1340 & 22.9 & 1410 & 24.1 & 58 & 11.7 & & 275 & $40.3 *$ & $5.7 \mathrm{E}-24^{\dagger}$ \\
\hline cell cycle & 65 & 1.1 & 68 & 1.2 & 6 & $1.2 *$ & $1.6 \mathrm{E}-01$ & 7 & 1 & \\
\hline reproduction & 130 & 2.2 & 137 & 2.3 & 6 & 1.2 & & 0 & 0 & \\
\hline intracellular organization & 195 & 3.3 & 201 & 3.4 & 27 & $5.4 *$ & $3.4 \mathrm{E}-03+$ & 54 & $7.9 *$ & $9.1 \mathrm{E}-10^{\dagger}$ \\
\hline development & 338 & 5.8 & 357 & 6.1 & 0 & 0 & & 0 & 0 & \\
\hline translation & 146 & 2.5 & 99 & 1.7 & 50 & $10.1 *$ & $4.4 \mathrm{E}-19^{\dagger}$ & 17 & $2.5 *$ & $3.9 \mathrm{E}-2{ }^{\dagger}$ \\
\hline death & 31 & 0.5 & 36 & 0.6 & 0 & 0 & & 3 & 0.4 & \\
\hline cytoskeleton & 57 & 1 & 59 & 1 & 0 & 0 & & 0 & 0 & \\
\hline ion transport & 10 & 0.2 & 8 & 0.1 & 2 & $0.4 *$ & $1.6 \mathrm{E}-01$ & 0 & 0 & \\
\hline proliferation & 3 & 0.1 & 2 & 0 & 1 & $0.2 *$ & $2.1 \mathrm{E}-01$ & 0 & 0 & \\
\hline homeostasis & 2 & 0 & 2 & 0 & 0 & 0 & & 0 & 0 & \\
\hline differentiation & 6 & 0.1 & 6 & 0.1 & 0 & 0 & & 0 & 0 & \\
\hline $\mathrm{NaN}$ & 161 & 2.7 & 179 & 3.1 & 0 & 0 & & 1 & 0.1 & \\
\hline Totals & 5855 & 100 & 5855 & 99.8 & 497 & 100 & & 683 & 99.9 & \\
\hline
\end{tabular}

* denotes an enrichment (increase in proportion) above that found in the appropriate "all networks in study" category (e.g., column 7 is compared to column 3, and column 10 is compared to column 5). + denotes a significant $p$-value $(p \leq 0.05)$.

\subsection{Genes Previously Implicated in Osteosarcoma Metastasis are among Significant Network Results}

Next, it was examined if the genetic networks discovered to be differentially activated or organized in expression profiles of MD-OS samples contained genes previously implicated in OS metastasis. Extensive literature review led to the generation of a query list containing genes whose expression is either correlated with outcome in human OS patients, or genes that have been shown to be involved in OS metastasis in xenograft studies or animal models of OS (Table 2). Thirty-two genes from this list were included in the present study (i.e., present on the expression profiling microarrays), and it was found that five such genes were among networks found to be significantly differentially activated in MD-OS expression profiles, and twenty-eight were among networks found to be significantly differentially organized in MD-OS expression profiles (Tables 3 and 4). 
Table 2. Genes previously implicated in metastatic progression of OS.

\begin{tabular}{ccccccc}
\hline Gene & Ref. & Gene & Ref. & Gene & Ref. \\
\hline BIRC5 & {$[16]$} & IVD & -- & CXCR3 & X & {$[17]$} \\
CAV1 & {$[18]$} & KIT & {$[19]$} & EGF & X & {$[20,21]$} \\
CCN1 & {$[22]$} & LRP5 & {$[23]$} & EGFR2 & X & {$[24-29]$} \\
CCN3 & {$[30]$} & MMP2 & {$[31]$} & FADD & X & {$[32]$} \\
CD44 & {$[33]$} & PDGFRA & {$[34,35]$} & HIF1 & X & {$[36]$} \\
CDH11 & {$[37]$} & PEDF & {$[38]$} & IL12A & X & {$[39]$} \\
CDH2 & {$[37]$} & RECK & {$[40]$} & IL12B & X & {$[39]$} \\
COL18A1 & {$[41]$} & S100A6 & {$[42]$} & INS & X & {$[43-51]$} \\
CXCR4 & {$[52-55]$} & SPARC & {$[56]$} & MAML1 & X & {$[57]$} \\
DPF2 & -- & TIMP1 & {$[58]$} & MIRK & X & {$[59]$} \\
EGFR & {$[60]$} & PLAUR & {$[61]$} & MMP14 & X & {$[62]$} \\
EZR & {$[25,63,64]$} & VEGFA & {$[55,65-72]$} & MMP9 & X & {$[58,73,74]$} \\
FAS & {$[32,75-77]$} & VEGFB & {$[55,65-72]$} & PDGFA & X & {$[35]$} \\
IGF1 & {$[43-51]$} & VEGFC & {$[55,65-72]$} & VCP & X & {$[78]$} \\
IGF2 & {$[43-51]$} & WIF1 & {$[79]$} & & & \\
IGF1R & {$[43-51]$} & YYI & {$[80]$} & & & \\
\hline
\end{tabular}

$\mathrm{X}$ denotes genes not present in the expression profiling microarrays used in this study.

Table 3. Differentially activated networks containing genes previously implicated in OS metastasis.

\begin{tabular}{ccccccc}
\hline Query & Node & Score & Psample & FDRsample & Pgene & FDRgene \\
\hline CDH2 & CDH2 & 3.89 & 0 & 0.0017 & 0.037 & 0.019 \\
S100A6 & CACYBP & 3.94 & 0 & 0.00096 & 0.007 & 0.18 \\
TIMP1 & ECH1 & 3.28 & 0 & 0.00096 & 0.003 & 0.17 \\
PLAUR & PGAP1 & 2.54 & 0 & 0.0019 & 0.003 & 0.19 \\
IVD & MECR & 3.94 & 0.001 & 0.01 & 0.046 & 0.0076 \\
\hline
\end{tabular}

"Node" is the central gene exhibiting differential network activity.

Table 4. Differentially organized networks containing genes previously implicated in OS metastasis.

\begin{tabular}{cccccccc}
\hline Query & Node & $\boldsymbol{\Delta}$ PCC $_{\text {Total }}$ & Psample & Query & Node & $\Delta$ PCC $_{\text {Total }}$ & Psample \\
\hline S100A6 & S100A6 & 0.41 & 0 & TIMP1 & LRP1 & 0.32 & 0 \\
KIT & KIT & 0.34 & 0 & PLAUR & LRP1 & 0.32 & 0 \\
YY1 & YY1 & 0.32 & 0.001 & BIRC5 & PAFAH1B1 & 0.34 & 0 \\
BIRC5 & BIRC5 & 0.35 & 0.001 & NOV & GIA1 & 0.3 & 0 \\
MMP2 & MMP2 & 0.35 & 0.001 & VEGFA & SPARC & 0.34 & 0 \\
SPARC & SPARC & 0.34 & 0 & YY1 & HDAC & 0.33 & 0 \\
IGF1R & CAMK2B & 0.37 & 0 & IGF1 & PRKCD & 0.36 & 0 \\
IGF2 & CAMK2B & 0.37 & 0 & PDGFRA & JAK1 & 0.33 & 0 \\
EGFR & CAMK2B & 0.37 & 0 & CXCR4 & JAK1 & 0.33 & 0 \\
KIT & CAMK2B & 0.37 & 0 & FAS & BTK & 0.35 & 0 \\
CDH2 & CAMK2B & 0.37 & 0 & VEGFB & RASA1 & 0.32 & 0 \\
EZR & CAMK2B & 0.37 & 0 & MMP2 & ITGB2 & 0.37 & 0 \\
CD44 & CAMK2B & 0.37 & 0 & LRP5 & FZD8 & 0.48 & 0 \\
SPARC & CAMK2B & 0.37 & 0 & SERPINF1 & CSNK2A1 & 0.33 & 0.001 \\
CYR61 & ATP2A2 & 0.33 & 0 & COL18A1 & CTSL1 & 0.33 & 0.001 \\
CAV1 & ATP2A2 & 0.33 & 0 & WIF1 & FZD1 & 0.37 & 0.001 \\
S100A6 & ACTN1 & 0.32 & 0 & IVD & MCCC1 & 0.37 & 0 \\
\hline
\end{tabular}


In order to identify drivers of the metastatic phenotype, the lists of significant networks discovered to be differentially activated and organized in expression profiles of MD-OS samples were further refined by focusing on a manageable number of each type of network that exhibited the greatest change between LD-OS and MD-OS samples. Twelve differentially organized networks were identified that exhibited the greatest change in organization between LD-OS and MD-OS samples, and that were also "genetic hubs" (having more interactors than the median), as it has been shown that these genes may be particularly important to cellular processes (Figure 3) [81]. Forty-three networks were identified that exhibited the greatest change in activity between LD-OS and MD-OS expression profiles, and their interactions with some of the most disorganized hubs were visualized (Figure 4). This analysis allowed the identification of the networks exhibiting the greatest change among MD-OS expression profiles, and also the observation that many differentially activated networks also interact with significantly disorganized hubs, e.g., the differentially activated network containing protein kinase $\mathrm{C}$ epsilon (PRKCE), RAS guanyl releasing protein 3 (RASGRP3), and guanine nucleotide binding protein 2 (GNB2), also interacts with the disorganized network of discs large homolog 2 (DLG2) (Figure 4).

Figure 3. Labeled black squares show 12 differentially organized hubs $(\triangle \mathrm{PCC} \geq 0.4$, $p=0$, interactors $\geq 7$ ). Nodes are genes and their colour reflects fold change in expression in the metastatic samples, only for significantly differentially expressed genes ( $\mathrm{t}$-test $p \leq 0.001$ ). Only interactors of a s ignificant hub whose expression is significantly correlated (PCC $p \leq 0.001$ ) with expression of that hub in either the localized or metastatic samples are included. Edges depict interactions, and their colour reflects change in co-expression between metastatic samples and localized samples (green: correlation increased in metastatic samples, purple: correlation decreased in metastatic samples). Asterisks denote co-expressions that are significant (PCC $p \leq 0.001)$ in both localized and metastatic samples.

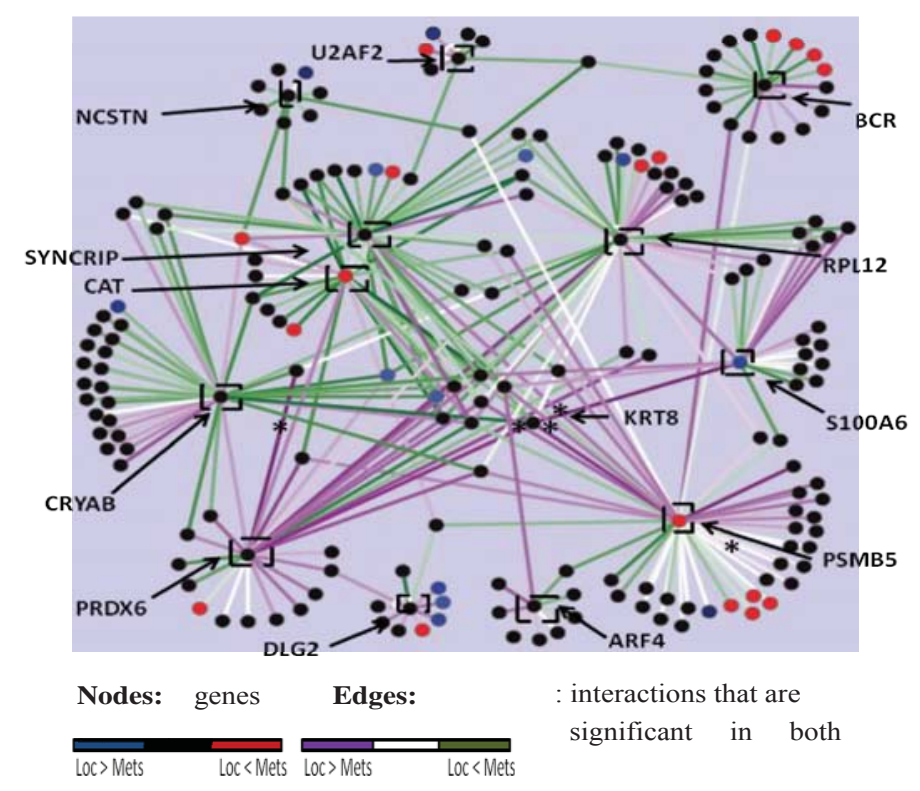


Figure 4. Genes comprising 43 di fferentially activated networks that meet stringent cut-offs (detailed at the top of the figure) were visualized with Cytoscape, as well as their interactions with 11 differentially organized networks (that also met stringent cut-offs which are detailed at the top of the figure). Coloured nodes represent relative abundance in metastatic samples $v s$. localized samples (red = more abundant, blue $=$ less abundant). Coloured edges represent correlations between genes in metastatic samples $v s$. localized samples $($ green $=$ more correlated, purple $=$ less correlated) . The number of included interactors of each gene in this study is proportional to the size of the node depicting the gene.

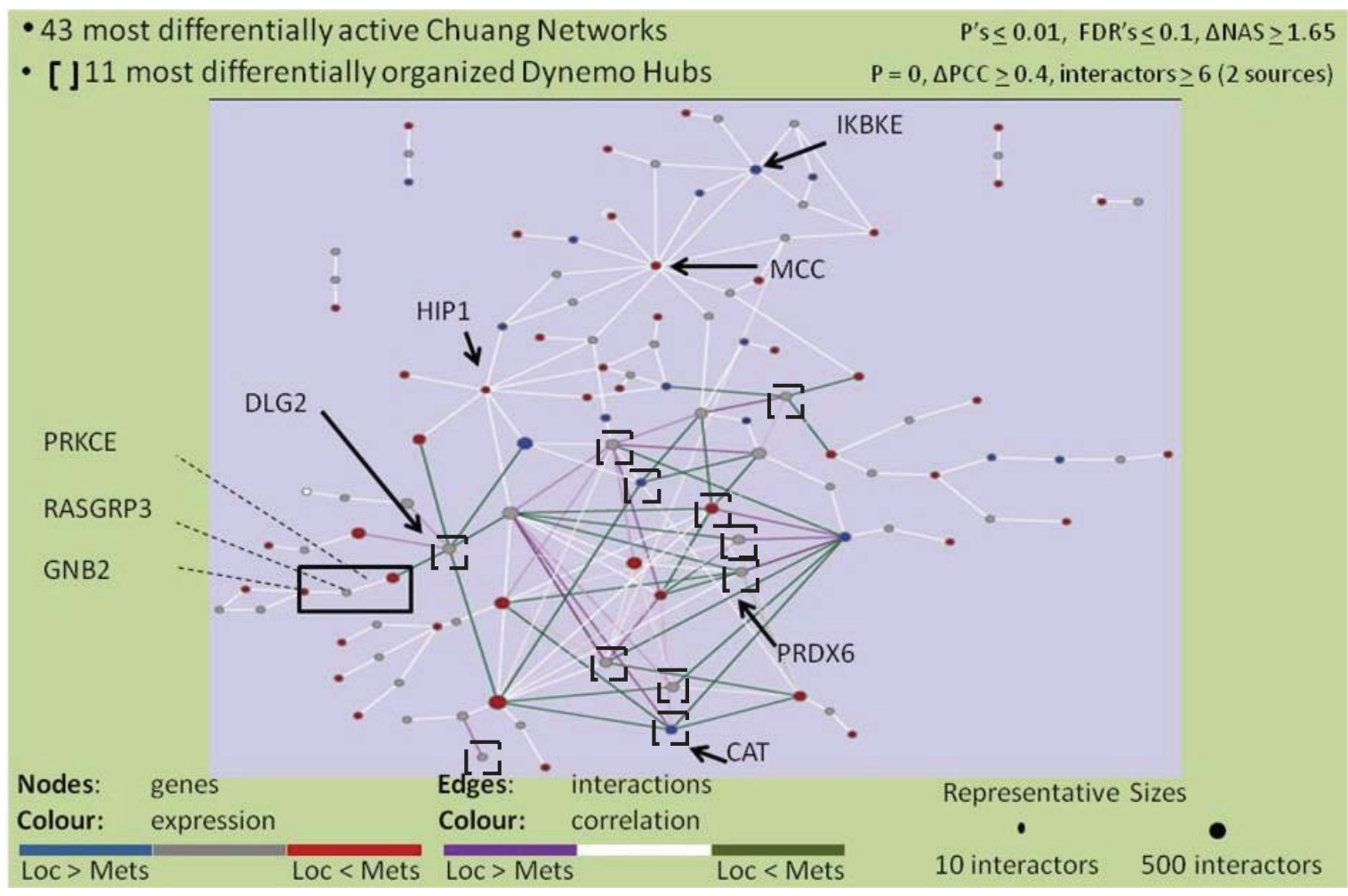

Literature searches of the genes contained in the highly significant network results showed that PRKCE has been characterized in progression of prostate [82-87], breast [88,89], and renal cancers [90], as well as tumorigenesis of squamous cell carcinoma (non-melanoma skin [91-95], and head and neck [96-99]), as well as non-small cell lung cancer [100-103]. Additionally high expression of GNB2 is associated with an aggressive form of pulmonary adenocarcinoma (mixed adenocarcinoma with bronchioalveolar features), and shorter overall survival for patients with these tumors [104]. RASGRP3 has been shown to promote androgen independence and progression of prostate cancer [105]. These reports of the involvement of these genes in the progression of other cancers led to the selection of the differentially activated PRKCE-RASGRP3-GNB2 network as a lead candidate for further characterization in osteosarcoma cells and tumors (Figure 5). 
Figure 5. (A) The PRKCe-RASGRP3-GNB2 Network is Differentially Activated in Expression Profiles of Metastatic Osteosarcomas. (B) Differential Activity Score of PRKCE-RASGRP3-GNB2 Network. (A) Shown is the network of PRKCE-RASGRP3-GNB2 that is differentially activated in metastatic OS samples of the expression profiling screen. Red nodes are transcripts that are more abundant in metastatic samples, and blue nodes are less abundant in metastatic samples. Within each circle is the \%-fold change in expression of metastatic samples $v s$. localized samples, and the order of magnitude of the $p$-value from the corresponding student's t-test. (B) Shown are the relevant scores for the PRKCE-RASGRP3-GNB2 network, the results of the differential network activity analysis applied to expression profiles of MD-OS $v s$. LD-OS tumors.

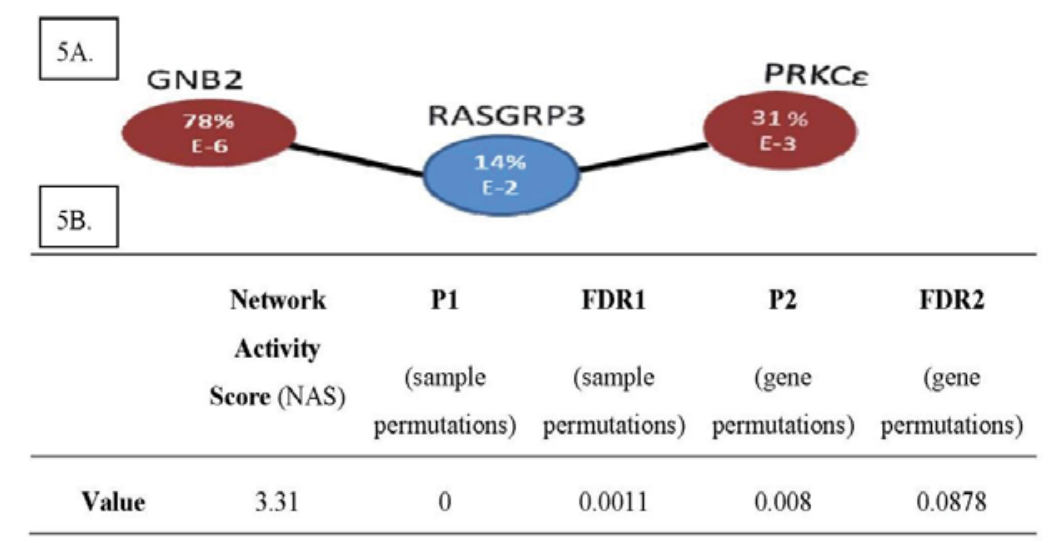

\subsection{PRKCE-RASGRP3-GNB2 Network Is Differentially Activated in Vitro}

A panel of human osteosarcoma cell lines known to have differing metastatic potential when grown as murine xenografts was collected to investigate the role of PRKCE-RASGRP3-GNB2. This panel includes the parental $\mathrm{Hu}-09$ cell line (included in Figure 6), and its highly metastatic derivatives M112 and M132 (derived by in vivo metastatic selection), as well as the poorly metastatic sub-clones L06 and L13 (all generously provided by Dr. B. Fuchs, the University of Zurich, Zurich, Switzerland) [106,107]. Additionally the poorly metastatic SAOS2 and MG63 cell lines, as well as their highly metastatic derivatives LM7 and M8, were included (generously provided by Dr. E. Kleinerman, University of Texas MD Anderson Cancer Center, Houston, TX, USA) [108,109]. In this panel, it was observed that the PRKCE-RASGRP3-GNB2 network exhibited an mRNA expression pattern similar to that observed in the expression profiles of osteosarcoma tumors (Figure 6). Specifically, it was observed that PRKCe mRNA was significantly more abundant in some highly metastatic lines (M112 and M132 vs. L06 and L13), and that RASGRP3 was significantly less abundant in some highly metastatic lines (LM7 vs. SAOS2 and M8 vs. MG63). Additionally, mRNA levels of myosin chain heavy 9 (MYH9), which has been shown to interact with PRKCE at stress fibers in mice [110], was also observed to be more abundant in some highly metastatic lines (LM7 vs. SAOS2 and M8 vs. MG63). The protein expression of PRKC $\varepsilon$ was also investigated in this panel, and it was found that the highly metastatic lines M112 and M132 expressed PRKC $\varepsilon$ protein at higher levels than observed in either the less metastatic parental Hu09 line, or in the poorly metastatic L13 derivative (Figure 7). 
Figure 6. The PRKCE-RASGRP3-GNB2 network is differentially activated in vitro. Quantitative PCR was performed on cDNA synthesized from the human OS cell lines shown. Purple bars denote cell lines that are poorly metastatic in mouse models, and green bars denote cell lines that are strongly metastatic as murine xenografts. Arrows denote relationships between cell lines. Student's t-test: ${ }^{*} p \leq 0.05 * * p \leq 0.001$. Multiple independent experiments are shown for each gene: GNB2 $(\mathrm{n}=2)$, PRKC $\varepsilon$ and RASGRP3 $(\mathrm{n}=3)$, and MYH9 $(\mathrm{n}=5)$. $* * p \leq 0.001, * p \leq 0.05$.
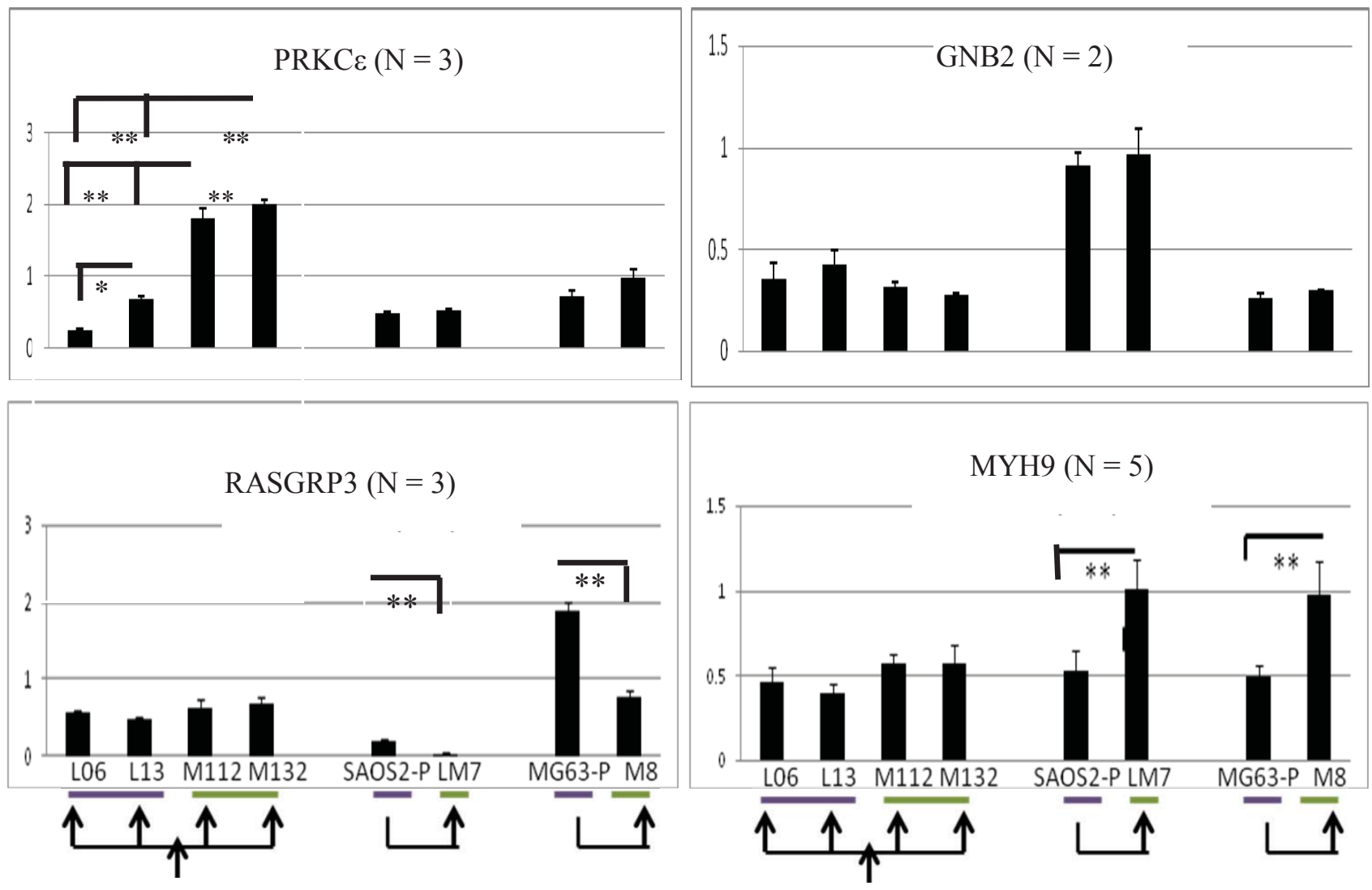

Figure 7. PRKCE Protein is more abundant in some highly metastatic cell line models of osteosarcoma metastasis. Western blots of whole cell lysates from human OS cell lines probed with anti-PRKC $\varepsilon$ and anti- $\beta$-actin antibodies. Purple bars denote cell lines that are poorly metastatic in mouse models, and green bars denote cell lines that are strongly metastatic in cell lines. Arrows denote relationships between cell lines. Shown is a representative western blot of two independent experiments.

\section{PRKCE protein}

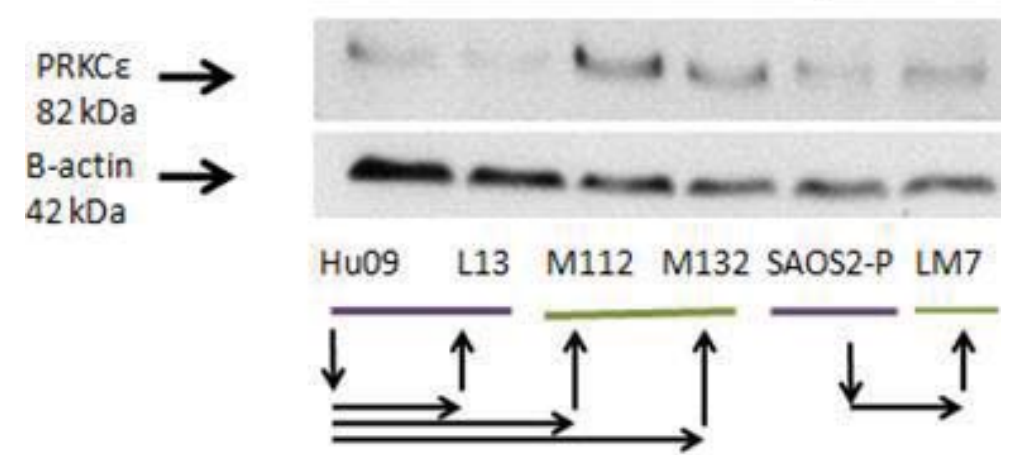


2.6. Human Osteosarcomas That Are Metastatic-at-Diagnosis Are More Likely to Exhibit High Levels of PRKCE $m R N A$

The amount of PRKC $\varepsilon$ mRNA was assayed in 17 LD-OS and 14 MD-OS tumors that were used in the expression profiling screen (Figure 8). It was found that MD-OS tumors do not have a higher average expression of PRKC $\varepsilon$ than LD-OS tumors (Welch two sample t-test $p=0.1873$ ), however MD-OS tumors were more likely to exhibit high expression of the PRKC $\varepsilon$ transcript (five of fourteen MD-OS tumors: Figure 8).

Figure 8. Metastatic-at-Diagnosis human osteosarcomas are more likely to have high PRKC $\varepsilon$ expression. Quantitative PCR was performed on cDNA synthesized from tumor samples from the original expression profiling cohort. PRKCE expression was normalized to STAM2, and is depicted as fold-change. The purple bar denotes localized tumors at diagnosis (LD-OS), and the green bar denotes metastatic tumors at diagnosis (MD-OS). The dotted red line depicts the average PRKCE expression in localized tumors. The number of replicates for each sample is shown below each bar.

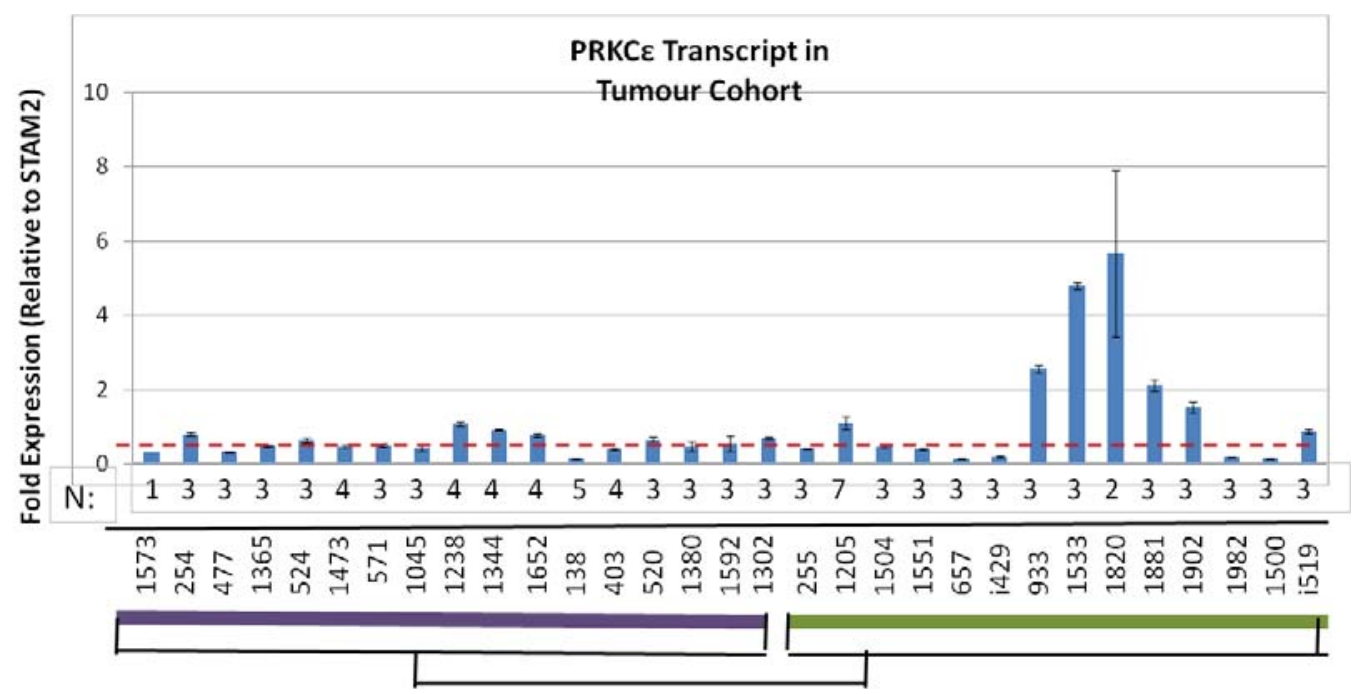

\subsection{PRKCE Is Not Required for Migration of Highly Metastatic M132 Cells}

Since PRKCE promotes the in vitro migration of other cell lines [89], and the in vitro migration rate of the Hu09-derived cell lines correlates with their ability to form metastatic colonies in mice [107], experiments were undertaken to de termine whether PRKCE promotes migration of osteosarcoma cells. The highly metastatic M132 cell line was selected for PRKCE-knockdown studies, as this line expressed PRKCe protein at a high level (Figure 7). However, it was observed that knockdown of PRKCE protein using siRNA did not affect the in vitro migration rates of M132 cells as observed during a scratch assay (Figure 9).

\subsection{IGF-1 Stimulation Induces Protein Expression of PRKCE in M112 Osteosarcoma Cells}

It is well known that the insulin/insulin-like growth factor (IGF) pathway plays an important role in osteosarcoma tumor growth. Osteosarcoma tumors and cell lines express components of the 
pathway (including insulin, IGF-I and -II, as well as pathway receptors) that are capable of autocrine signaling $[44,45,51]$. Additionally, inhibition of the pathway through various means is effective at inhibiting osteosarcoma growth in xenograft models [43,46-50]. IGF-I signaling is also known to lead to increased cellular levels of diacylglycerol, which can then activate PRKC $\varepsilon$ (and other proteins containing C1 domains) [111-113], by a mechanism involving membrane tethering of PRKCE and conformational change [114]. Specifically PRKC $\varepsilon$ is activated following IGF-1 treatment in vascular smooth muscle cells, and may be involved in IGF-I-mediated proliferation and migration of these cells $[112,113]$.

Figure 9. (a) PRKCE is not required for in vitro migration of highly metastatic M132 osteosarcoma cells. (b) Confirmation of PRKCE knockdown. (c) Quantification of migration by M132 cells. (a) Upper panel: photographs of M132 cells immediately after scratching, two days following transfection with PRKCE siRNA. Lower panel: matched photographs of M132 cells two days following scratching and four days following transfection with PRKCE siRNA. (b) Western blot verification of PRKCE knock-down two, three and four days following transfection with siRNA. (c) Quantification of migrated distance by M132 cells transfected with PRKCE siRNA and appropriate controls. Values are average \pm standard error of three independent experiments.

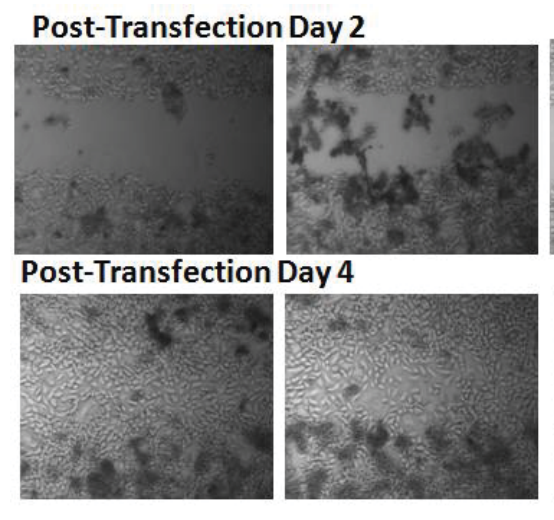

Day 2

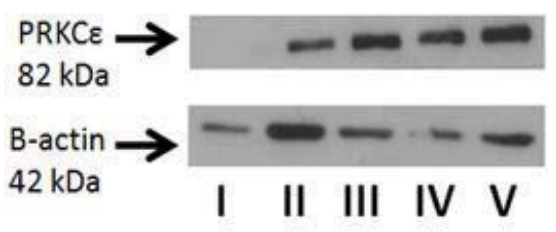

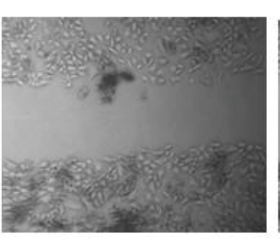

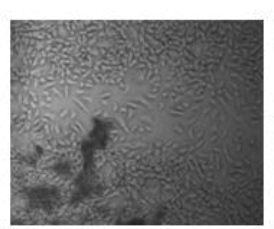

(a)

Day 3

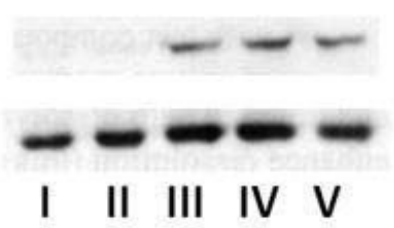

(b)

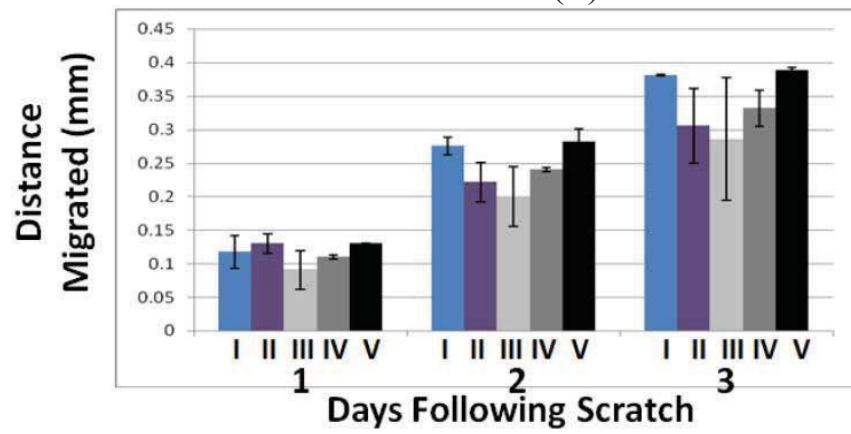

(c)
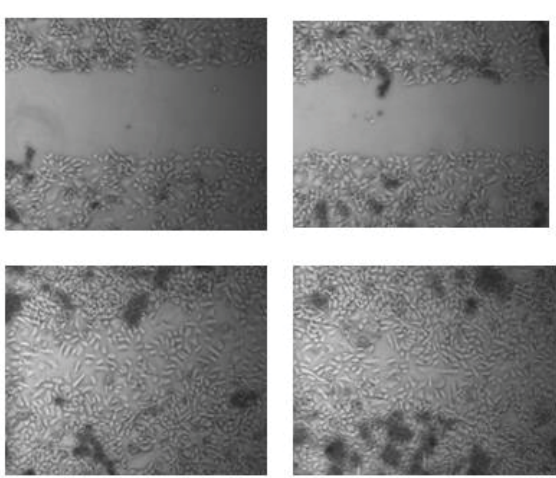

Day 4

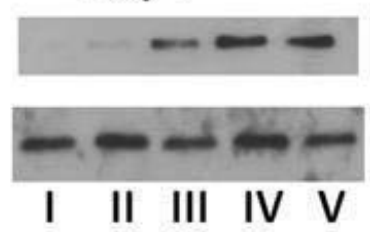

Legend

I PRKC $\varepsilon$ SIRNA \#2

II PRKCE SIRNA \#3

III Scramble

IV Mock

V Cells 
The effect of IGF-1 stimulation on PRKCe protein in highly metastatic osteosarcoma cells was investigated. Highly metastatic M112 osteosarcoma cells were serum starved for at least $24 \mathrm{~h}$, followed by the addition of either fresh serum-free media or media containing $50 \mathrm{ng} / \mathrm{mL}$ IGF-1. It was observed that following IGF-1 stimulation, the protein expression of PRKC $\varepsilon$ increased in a time-dependent manner, with a peak occurring approximately $30 \mathrm{~min}$ following stimulation (Figure 10).

Figure 10. Protein expression of PRKC $\varepsilon$ is induced by IGF-1 treatment. Western blots were performed on whole cell lysates of M112 cells incubated in 1\% FBS (low serum) medium or in 1\% FBS medium supplemented with $50 \mathrm{ng} / \mathrm{mL}$ IGF-1 for the time periods described. Membranes were probed with anti-PRKC $\varepsilon$ antibody and anti- $\beta$-actin antibody as a loading control. The experiment was performed three independent times.

\section{M112 cell line $(\mathrm{N}=3)$}

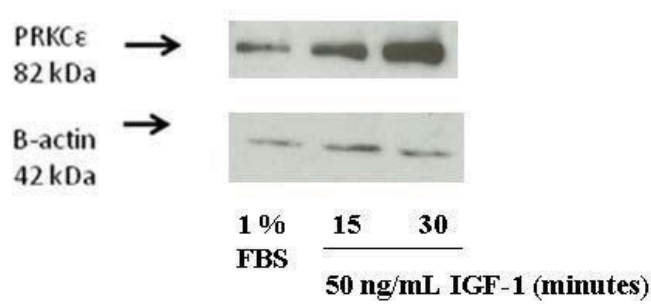

\section{Discussion}

This work demonstrates that expression profiles of metastatic-at-diagnosis osteosarcomas (MD-OS) are quite distinct from expression profiles of localized-at-diagnosis osteosarcomas (LD-OS), possibly indicating a distinct disease etiology for the more severe MD-OS. Supervised network analysis discovered hundreds of networks that exhibited both differential activity and differential organization in the MD-OS expression profiles, at permissive cut-off levels. This indicates that either heterogeneous differences are observed in the MD-OS tumor group, or that changes to the transcriptome observed in MD-OS are potentially associated with many bystander events (i.e., aberrations in expression pattern not functionally relevant to osteosarcoma metastasis), or possibly both.

Investigation of the cellular processes of networks within the permissive significant results showed differentially activated networks were strongly enriched for transport and translation networks, and slightly enriched for intracellular organization networks. Differentially organized networks were strongly enriched for metabolic networks, as well as intracellular organization and transport networks. Increased or over-active translation is known to support an aggressive phenotype of many cancers [115], and the analysis presented here implies it is a characteristic of MD-OS tumors as well. The enrichment of disorganized metabolic networks in MD-OS expression profiles may indicate that altered cellular metabolism plays a role in osteosarcoma metastasis, a notion supported by a recent study by Hua et al. [116]. By studying serum metabolite profiles of mice injected with OS cells, Hua et al observed a metabolic shift coincident with the onset of pulmonary metastasis [116].

Examination of the most stringently differentially activated and organized networks in MD-OS led to the selection of the PRKCE-RASGRP3-GNB2 network for follow-up characterization. This 
network was subsequently found to be differentially activated at the transcript level among a panel of in vitro models of OS metastasis. PRKCE was also found to be more abundant in some highly metastatic OS cells at the protein level, and in some MD-OS tumors at the transcript level. Although PRKC $\varepsilon$ is known to support migration of other cell types [89,96,98,117,118], there was no evidence to support a role for PRKCe in the migration of M132 osteosarcoma cells in this study. This may indicate that the aberrant expression of PRKCE and its network in vitro and in vivo is related to a bystander effect, or that the functional relevance of this aberrant expression has not yet been elucidated. The effect of high PRKCE expression may be related to the proliferation effects of the IGF-1 pathway, as this study provides evidence for the IGF-1 dependent induction of PRKC $\varepsilon$ protein expression. PRKCE may support other cellular processes entirely, as it is known to promote pro-metastatic phenotypes of many other cancers [82-87,91-103].

In addition to the PRKCE-RASGRP3-GNB2 and MYH9 network; this article describes several networks exhibiting significant differential activity, organization, or some combination of the two in MD-OS expression profiles. The integration of the results of this study with other datasets of osteosarcoma expression profiles, as they become available, will help to distinguish the drivers and genuine characteristics of metastatic osteosarcoma from the passengers, as would a 1 imited high-throughput functional screen of the significant networks described in this study.

\section{Experimental Section}

\subsection{Patient Follow-Up}

Overall survival data was available for all 46 patients in the group presenting without metastasis at diagnosis. Of these, 17 died of disease (DOD) with a median follow-up of 41 months (minimum follow-up $=6$ month, maximum follow-up $=157, \mathrm{SD}=39.2$ months), 28 are alive with no evidence of disease (ANED), with a median follow-up of 101 months (minimum follow-up $=35$ month, maximum follow-up $=269, \mathrm{SD}=62.1$ months) and one subject is alive with evidence of disease (AWED) with a follow-up of 12 months.

Out of 17 pat ients presenting with metastases, overall survival data was available for only 14 patients. Out these 14, $13 \mathrm{~d}$ ied of disease (DOD) with a median follow-up of 10 months (minimum follow-up $=1$ month, maximum follow-up $=49, \mathrm{SD}=12.5$ months) and the other patient is alive with no evidence of disease (ANED) with a follow-up of 178 months.

\subsection{Tumor Samples}

Primary high-grade intramedullary osteosarcoma tumors were selected for expression profiling by sarcoma pathologists on the basis of tumor homogeneity. The OS tumor cohort consisted of 63 tumors which were grouped into those that were localized $(n=46)$ or metastatic $(n=17)$ at the time of initial diagnosis. Samples were collected by open biopsies prior to administration of any chemotherapy, and stored in liquid nitrogen until time of RNA isolation. Total RNA was extracted using Trizol reagent (Invitrogen, Carlsbad, CA, USA). The amount and quality of RNA was assessed using both Ultrospec 2100 pro (GE Healthcare Bio-Sciences, Piscataway, NJ, USA) and $1 \%$ agarose gels. 


\subsection{Gene Expression Profiling}

$5 \mu \mathrm{g}$ of tumor and "reference" (pooled from cell lines) cDNA was indirectly labeled using aminoallyl nucleotide analogues with Cy3 and Cy5 fluorescent tags, respectively. The labeled cDNA was competitively hybridized to University Health Network 19 k cDNA arrays (UHN19k) containing 18,981 "spots" (mapped to 8,998 known unique genes). This process was repeated with reciprocal fluorescent tagging. Data normalization, imputation (K10 Nearest Neighbours algorithm), and analysis were performed in collaboration with Drs. Shelley Bull, Dushanthi Pinnaduwage, and Robert Parkes. Supervised statistical analysis (random variance T-test) was performed by Robert Parkes using BRB-Array Tools software [119].

\subsection{Unsupervised Hierarchical Clustering}

The most differentially expressed single genes within the expression profiling experiment were identified according to methods previously described $[13,14]$. In this study, a subset was examined that exhibited at least six-fold change in expression in at-least four tumors with a maximum $p$-value of 0.001 (student's t-test). Unsupervised hierarchical clustering of the tumors according to their expression of these genes was performed with Partek Genomics Suite.

\subsection{Supervised Network Analysis}

The entire database of interactions for human genes and proteins was downloaded from the Pathway Commons website (as an adjacency list and the interactions were converted to Entrez GeneIDs [15]. This dataset comprised physical and genetic interactions, as well as pathway and disease associations (which were either translated by Pathway Commons to binary interactions or were lost), from both curated and non-curated sources [15]. A subset of 5,855 genes was common to both this interaction database and the expression profiling experiment. There were 176,121 interactions among these genes, with six interactions being the median number per gene.

\subsubsection{Differentially Activated Networks}

Genetic networks demonstrating significant differential "activity" in MD-OS tumors were discovered in a manner similar, with some changes, to that previously described by Chuang et al. [11]. Briefly each network was restricted to those genes that met some cut-off of significant expression between localized and metastatic tumors (Table 5).

Table 5. Cut-Off conditions for gene inclusion to "differential activity" analysis.

\begin{tabular}{ccccccccccc}
\hline Trial & $\mathbf{1}$ & $\mathbf{2}$ & $\mathbf{3}$ & $\mathbf{4}$ & $\mathbf{5}$ & $\mathbf{6}$ & $\mathbf{7}$ & $\mathbf{8}$ & $\mathbf{9}$ & $\mathbf{1 0}$ \\
\hline $\begin{array}{c}\text { Differential } \\
\text { Expression (\%) }\end{array}$ & 0 & 0 & 10 & 20 & 30 & 40 & 50 & 60 & 70 & 80 \\
\hline P Value Maximum & None (i.e., all genes included) & \multicolumn{7}{c}{0.001} \\
\hline
\end{tabular}

For each gene in the remaining network a "class difference score" (CDS) was calculated as the difference in median expression between classes (i.e., between metastatic tumors and localized tumors), and normalized to the variation within the localized samples [Equation (1)]. A "network 
activity score" (NAS) was calculated by determining the average of the absolute CDS for each gene in the network [Equation (2)]. In these equations, $\mathrm{g}_{1}, \mathrm{~g}_{2} \ldots \mathrm{gn}$ are all genes in network $\mathrm{J}$, which has $\mathrm{N}_{\mathrm{G}}$ members. $\mathrm{G}_{\mathrm{A}}$ and $\mathrm{G}_{\mathrm{B}}$ are the median expression of gene $\mathrm{G}$ among localized (A) and metastatic (B) tumors. $\mathrm{S}_{\mathrm{GA}}$ is the standard deviation of expression values of gene $\mathrm{G}$ among the localized tumors:

$$
\begin{gathered}
\mathrm{CDS}_{G}=\frac{\tilde{\mathrm{G}}_{B}-\frac{\tilde{\mathrm{G}}_{A}}{\mathrm{~S}_{\mathrm{GA}}}}{N A S_{J}=} \\
\frac{\sum_{\mathrm{g} 11}^{\mathrm{gn}}\left|\mathrm{CDS}_{G}\right|}{N_{G}}
\end{gathered}
$$

To determine statistical significance, the NAS was compared to the corresponding NAS generated from 1,000 permutations of both sample and gene labels to determine two empirical $p$-values [Equation (3)]. In these equations $\mathrm{J} 1 \ldots \phi-1, \phi, \phi+1 \ldots \hat{\mathrm{J}}$ are all networks in the study, and $\mathrm{i} \ldots \mathrm{l}-$ $1,1,1+1 \ldots \mathrm{N}_{\mathrm{i}}$ are the repetitions of gene and label permutations (in this study $\mathrm{N}_{\mathrm{i}}=1,000$ ).

$$
\begin{gathered}
f= \begin{cases}0 & \text { if } N A S_{\jmath}>N A S-R_{j i} \\
1 & \text { if } N A S_{\jmath} \leq N A S-R_{j i}\end{cases} \\
P_{J}=\frac{\sum_{\substack{j=\hat{\jmath} \\
i=1 \ldots N_{i}}} f\left(N A S_{\jmath}, N A S-R_{j i}\right)}{N_{i}}
\end{gathered}
$$

The significance of the randomly generated NAS scores was also determined in a similar fashion [Equation (4)]; this was done for false-discovery rate (FDR) calculation.

$$
\begin{aligned}
& h= \begin{cases}0 & \text { if } N A S_{\jmath_{\dagger}}>N A S-R_{j i} \\
1 & \text { if } N A S_{\jmath+} \leq N A S-R_{j i}\end{cases} \\
& P_{j t}=\sum_{\substack{j=\hat{j} \\
t-1+t+1}} h\left(\text { NAS }_{j t}, \text { NAS- } R_{j j}\right) \\
& \mathrm{N}_{\mathrm{i}}-1
\end{aligned}
$$

Two FDRs were calculated for each network by determining the average number of randomly-generated networks with a $p$ value equal to or lower than a nominal $p$ value threshold $\left[p_{\phi}\right.$ in Equation (5) below], equal to the $p$-value of the network being considered. The number of randomly generated $p$-values falling below this nominal threshold was then divided by the number of real networks also falling below this nominal threshold [Equation (5)]. This was done for both $p$-values (from sample and gene permutations) to yield two empirical FDR values for each network. The entire process was repeated for different cut-off conditions (Table 5), and was stopped when more stringent cutoffs failed to produce any additional significant networks:

$$
\begin{aligned}
& k=\left\{\begin{array}{ll}
1 & \text { if } P_{J} \leq P_{\phi} \\
0 & \text { if } P_{J}>P_{\phi}
\end{array} \quad m= \begin{cases}1 & \text { if } P_{J t} \leq P_{\phi t} \\
0 & \text { if } P_{J t}>P_{\phi t}\end{cases} \right.
\end{aligned}
$$

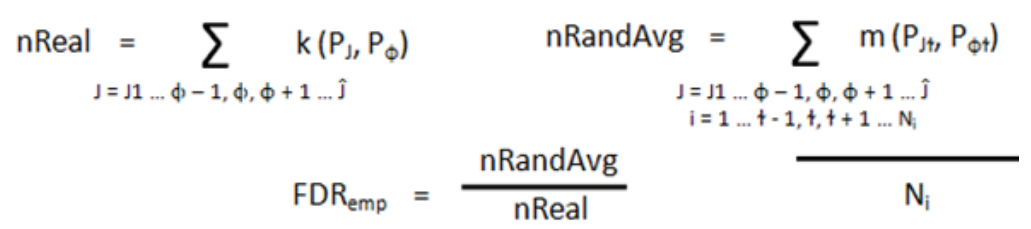




\subsubsection{Differentially Organized Network—Dynemo}

Genetic networks exhibiting significant differential organization in MD-OS samples relative to localized samples were discovered as previously described [12]. Briefly, for each network the Pearson Correlation Coefficient (PCC) was used to determine the overall correlation in gene expression between the network hub and each of its interactors in both the localized and metastatic samples [Equation (6)]. The difference in this hub-interactor correlation between localized and metastatic samples was then calculated for each interaction in the network [Equation (7)], and the average difference in correlation across the entire network was also calculated: "Avg $\Delta \mathrm{PCC}$ " [Equation (8)]. In these equations $\mathrm{J} 1 \ldots \phi-1, \phi, \phi+1 \ldots \hat{\mathrm{J}}$ are all the networks in the study. $\mathrm{H}$ is the hub (central node) of network $\mathrm{J}$, which has $\mathrm{N}_{\mathrm{G}}$ members, and $\mathrm{g} 1, \mathrm{~g} 2 \ldots$ gn are all interactors of $\mathrm{H}$, and therefore all other genes in network $J . S_{G}$ and $S_{H}$ are the standard deviations of gene $G$ and hub $H$ among the indicated tumor class. $\mathrm{t} 1, \mathrm{t} 2 \ldots \mathrm{T}$ are all the tumors in each class, and $\mathrm{N}_{\mathrm{T}}$ is the total number of tumors in the localized $\left(\mathrm{N}_{\mathrm{T}}=46\right)$ and metastatic $\left(\mathrm{N}_{\mathrm{T}}=17\right)$ classes:

$$
\begin{gathered}
\mathrm{PCC}_{\mathrm{g}}=\frac{\sum_{t=\mathrm{T}}\left(\mathrm{G}_{\mathrm{t}}-\overline{\mathrm{G}}\right)\left(\mathrm{H}_{\mathrm{t}}-\mathrm{H}\right)}{\left.{ }^{\prime} \mathrm{N}_{\mathrm{T}}-1\right) \mathrm{S}_{\mathrm{G}} \mathrm{S}_{\mathrm{H}}} \\
\Delta \mathrm{PCC}_{\mathrm{g}}=\mathrm{PCC}_{\mathrm{g}-\text { Metastatic }}-\mathrm{PCC}_{\mathrm{g} \text {-Localized }} \\
\text { Avg } \Delta \mathrm{PCC}=\frac{\sum_{\mathrm{g}=\mathrm{g} 1 \ldots \mathrm{gn}}\left|\Delta \mathrm{PCC}_{\mathrm{g}}\right|}{\mathrm{N}_{\mathrm{G}}}
\end{gathered}
$$

The Avg $\triangle \mathrm{PCC}$ value was compared to the corresponding scores generated from the same network following 1,000 permutations of the class labels to generate a non-parametric $p$-value to assess the significance of the change in internal correlation of each network [Equation (9)]. Let i ... $1-1,1$, $1+1 \ldots N_{i}$ be the number of repetitions of gene and label permutations (in this study $N_{i}=1,000$ ).

$$
\begin{aligned}
& q= \begin{cases}0 & \text { if Avg } \triangle P C C_{j}>A v g \Delta P C C-R_{j i} \\
1 & \text { if Avg } \triangle P C C_{j} \leq A v g \Delta P C C-R_{j i}\end{cases} \\
& P_{J}=\sum_{\substack{j=\hat{j} \\
i=1 \ldots N_{i}}} q\left(A v g \Delta P C C_{j}, A v g \Delta P C C-R_{j i}\right) \\
& N_{i}
\end{aligned}
$$

\subsubsection{Visualization of Network Results}

Two statistical confidence levels were investigated in this study, a permissive cut-off level to discover broad (or "global") trends among metastatic tumors, and a stringent cut-off to delineate high-confidence networks for follow-up. The permissive cut-off for differentially activated networks was chosen to be $p$ 's $\leq 0.05$ and FDR's $\leq 0.2$, and for the differentially organized levels the permissive cut-off was $p \leq 0.001$. These cut-offs were used to assess the significance of cellular process enrichment at the global level (Table 1) and for discovery of networks containing genes previously implicated in OS metastasis (Tables 3 and 4). Visualization of these global trends (Figure 1) was limited to computer processing power, and thus were further refined for the differentially activated networks to be $p$ 's $=0.05$, FDR's $=0.1$ and NAS $\geq 1.65$, and for differentially 
organized networks to be $p \leq 0.001$ and $|\Delta \mathrm{PCC}| \geq 0.4$. Visualization of the global trends was performed with the Enrichment Map plugin for Cytoscape [120]. The stringent cut-off levels for differentially activated networks were set to $p$ 's $\leq 0.01$, FDR's $\leq 0.1$, NAS $\geq 1.65$, and for differentially organized networks were set to $p=0,|\Delta \mathrm{PCC}| \geq 0.4$, and having at least seven interactors. Visualization of these high-confidence networks was conducted using Cytoscape [121] (Figures 3 and 4).

\subsubsection{Cellular Process Annotation}

Gene Ontology annotations (which relate genes to cellular processes) from the "Generic Slim" database were downloaded [122]. The database was further simplified to focus on interesting processes according to Table 6. Networks were assigned to processes by determining the most commonly occurring term among genes within the network. In this manner all 5,855 networks in the study could be assigned to a process (Figure 11a - the "study process composition", or Table 1, column 4). Since analysis of "differential activated" networks requires identification of significant subsets, all networks which were eventually found to be significantly differentially activated were re-annotated using only the genes within the network's significant subset (Table 1, column 2). This resulted in discordant annotations between the network and the significant subset for only $6 \%$ of all networks, and thus the annotations of the network subsets (Figure 11b- "subsets process composition", or Table 1, column 2) and overall "study process composition" (Figure 11a, or Table 1, column 4) are overwhelmingly similar. As the methods of Taylor et al. do not identify significant subsets within networks, this consideration was not necessary for differentially organized networks.

Table 6. Simplification of the gene ontology slim generic database.

\begin{tabular}{ll}
\hline \multicolumn{1}{c}{ Original Terms } & Further Simplified Terms \\
\hline cell death, death & death \\
\hline $\begin{array}{l}\text { multicellular organismal development, embryonic } \\
\text { development, anatomical structure morphogenesis }\end{array}$ & development \\
\hline cell differentiation, differentiation & differentiation \\
\hline regulation of gene expression, epigenetic & epigenetics \\
\hline cell growth, growth & growth \\
\hline $\begin{array}{l}\text { cellular component organization, organelle organization, } \\
\text { mitochondrion organization, cytoplasm organization }\end{array}$ & intracellular organization \\
\hline $\begin{array}{l}\text { metabolic process, cellular amino acid and derivative metabolic } \\
\text { process, secondary metabolic process, lipid metabolic process, } \\
\text { biosynthetic process, catabolic process, carbohydrate metabolic } \\
\text { process, protein metabolic process, nucleobase nucleoside } \\
\text { nucleotide and nucleic acid metabolic process, DNA metabolic } \\
\text { process, generation of precursor metabolites and energy }\end{array}$ & \\
\hline $\begin{array}{l}\text { signal transduction, response to biotic stimulus, response to external } \\
\text { stimulus, response to abiotic stimulus, cell-cell signaling, cell } \\
\text { communication, response to endogenous stimulus, cell recognition }\end{array}$ & signaling \\
\hline protein transport, transport & transport \\
\hline \begin{tabular}{l} 
regulation of biological process, biological process, behavior \\
\hline
\end{tabular} & NaN \\
\hline
\end{tabular}


Figure 11. Distribution of cellular processes among networks and sub-networks investigated in this study. (a) "Study" Process Composition (b) "Subsets" Process Composition. (a) The Generic Slim database of gene-process associations was accessed from Gene Ontology and each network was annotated according to the most commonly occurring process term among genes within the network. (b) For all 497 networks found to be differentially activated in this study, the networks were re-annotated according to the most commonly occurring term among the significant sub-set (as the analysis of differential activity involves identification of a significant subset of genes within each network).

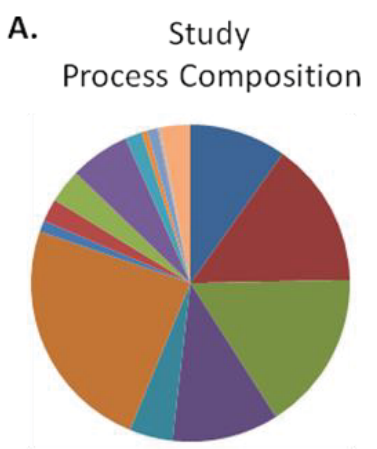
B. Subsets Process Composition

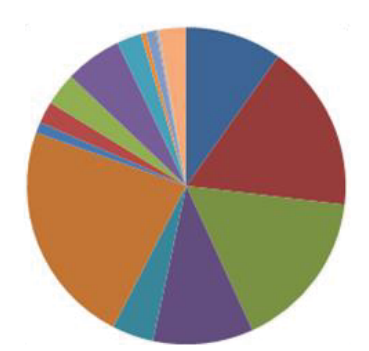

\begin{tabular}{|c|c|}
\hline protein modification & a development \\
\hline a transport & E translation \\
\hline = signalling & = death \\
\hline = transcription & cytoskeleton \\
\hline stress & Eion transport \\
\hline metabolism & proliferation \\
\hline cell cycle & womeostasis \\
\hline reproduction & differentiation \\
\hline intracellular organization & $\equiv \mathrm{NaN}$ \\
\hline
\end{tabular}

4.5.5. Cellular Process Enrichment

Enriched cellular processes were determined separately for differentially activated and organized networks. Enriched processes were first identified by determining those processing comprising a greater proportion within the significant results than in the entire study (i.e., differentially activated: Table 1 column 7 was compared to Table 1 column 3, differentially organized: Table 1 column 10 was compared to Table 1 column 5).

Significance of cellular process enrichment ( $p$-value) within the network results was determined using the hypergeometric distribution [Equation (10)], as described by Boyle et al. [123]. In this equation, $\mathrm{N}$ is the total number of networks in the study $(5,855)$. $\mathrm{M}$ is the number of networks in the entire study annotated to a p rocess of interest (differentially activated: Table 1 co lumn 2, differentially organized: Table 1 column 4). $i$ is the number of networks within the significant results annotated to the same process of interest (differentially activated: Table 1 column 6 , differentially organized: Table 1, column 9), and $\mathrm{n}$ is the number of networks contained within that set of significant results (bottom of Table 1-differentially activated: 497, differentially organized: 683). This operation was performed with Matlab using the "hygepdf" command.

$$
\mathrm{P}=1-\sum_{i=\mathrm{C}}^{\mathrm{k}-1} \frac{\left(\begin{array}{c}
\mathrm{M} \\
i
\end{array}\right)\left(\begin{array}{c}
\mathrm{N}-\mathrm{M} \\
\mathrm{n}-i
\end{array}\right)}{\left(\begin{array}{c}
\mathrm{N} \\
i
\end{array}\right)}
$$

\subsection{Cell Culture}

Hu09 and derivates (L06, L13, M112 and M132) were grown in RPMI 1640 supplemented with $10 \%$ fetal bovine serum (FBS) and 1\% L-glutamine. L06 and L13 are cell lines derived from Hu09 
human OS cell line by limited dilution plating, and M112 and M132 were derived from the Hu09 line by in vivo selection of pulmonary metastatic nodules [106,107]. L06 and L13 both form fewer lung metastases at a decr eased incidence in mice following tail-vein injection compared to the intermediate Hu09 line, while M112 and M132 result in greater numbers of pulmonary nodules at a higher incidence [107]. The differences in metastatic propensity correlate to di fferent survival lengths for mice injected with the various cell lines [107]. M8 cells were derived from MG63 human OS ce lls (hereafter referred to as MG63-P), and have decreased latency until pulmonary metastasis [109]. Both were grown in Dulbecco's Modified Eagle Medium supplemented with 10\% FBS. LM7 cells were derived from SAOS2 human OS cells (hereafter referred to as SAOS2-P), and form pulmonary metastases at a greater incidence than SAOS2-P following tail-vein injection in mice [108]. LM7 and SAOS2-P were grown in McCoy's 5A supplemented with 5\% FBS. RNA was harvested from all cell lines using Trizol reagent followed by phenol/chloroform extraction.

\subsection{Quantitative Reverse-Transcription Polymerase Chain Reaction (rt-PCR)}

RNA was collected from OS tumor samples as previously described [124], and cDNA was synthesized from both tumor RNA and cell line RNA according to the manufacturer's instructions (M-MLV Reverse Transcriptase-Invitrogen, Carlsbad, CA, USA). Quantitative rt-PCR was performed according to the manufacturer's instructions (Sybr Green-Applied Biosystems, Life Technologies, Carlsbad, CA, USA) to quantify the abundance of target cDNA relative to that of a control gene, signal transducing adaptor molecule 2 (STAM2), using primer sequences according to Table 7. Dr. Dushanthi Pinnaduwage performed statistical analysis (Welch two-sample t-test on log2 transformed data) comparing expression levels of protein kinase $\mathrm{C}$ epsilon (PRKCE) transcript between localized and metastatic tumors.

Table 7. Primer sequences used in this study.

\begin{tabular}{|c|c|}
\hline Gene Symbol & Primer Pairs \\
\hline \multirow[t]{2}{*}{ STAM2 } & Forward 5'-TGGATGACAGTGATGCCAATTG-3' \\
\hline & Reverse 5'-CGCTGCCTCAGTCTCTATGT-3' \\
\hline \multirow[t]{2}{*}{ PRKC $\varepsilon$} & Forward 5'-CACTGCAAGCTGGCTGACT-3' \\
\hline & Reverse 5'-TGCAGGATCTCAGGAGCTATG-3' \\
\hline \multirow[t]{2}{*}{ RASGRP3 } & Forward 5'-GGATTTCTCTGGGGCATAATC-3' \\
\hline & Reverse 5'-AGGAGGTCTTTGCACTGTTTG-3' \\
\hline \multirow[t]{2}{*}{ GNB2 } & Forward 5'-CTATCAAGCTGTGGGACGTG-3' \\
\hline & Reverse 5'-GTAGCCGTTGGGGAAGAAAG-3' \\
\hline \multirow[t]{2}{*}{ MYH9 } & Forward 5'-GCCTACAGGAGTATGATGCAAG-3' \\
\hline & Reverse 5'-ACTGGATGACCTTCTTGGTGTT-3' \\
\hline
\end{tabular}

\subsection{Western Blots}

Cytosolic protein extracts were isolated from cell lines using NETN lysis buffer $(150 \mathrm{mM} \mathrm{NaCl}$, $1 \mathrm{mM}$ EDTA, $20 \mathrm{mM}$ Tris pH 7.5, 0.5\% NP40, $1 \mathrm{mM}$ phenylmethylsulphonyl fluoride, and 1\% each of protease inhibitor, phosphatase inhibitor I and phosphatase inhibitor II, all from Sigma-Aldrich, (St. Louis, MO, USA). Protein concentration was determined using the bicinchoninic acid (BCA) 
protein assay kit (Pierce, Thermo Scientific, Rockford, IL, USA). Proteins were separated using 10\% sodium dodecyl sulfate polyacrylamide gel electrophoresis (SDS-PAGE) and transferred to nitrocellulose membranes at $30 \mathrm{~V}$, overnight at $4{ }^{\circ} \mathrm{C}$. Membranes were blocked for $1 \mathrm{~h}$ with Tris buffered saline with $0.1 \%$ Tween-20 (TBS-T) supplemented with 5\% fat-free milk. Primary antibody incubation was performed in TBS-T supplemented with $5 \%$ bovine serum albumin (BSA) or fat-free milk, according to the manufacturer's instructions. Secondary antibody incubation (donkey anti mouse, donkey anti rabbit) was performed at 1:5,000 concentration in TBS-T with 5\% BSA for 50 min. Protein bands were visualized by chemiluminescence using ECL detection system (Amersham, GE Healthcare Bio-Sciences Corp, Piscataway, NJ, USA,). Primary antibodies used in this study are PKC $\varepsilon(1: 1,000$, Cell Signaling Technology, Danvers, MA, USA), and $\beta$-actin (1:5,000, Sigma-Aldrich, St. Louis, MO, USA). Analysis of PRKCE protein expression in the panel of OS cell lines was performed in triplicate.

\subsection{Knockdown of PRKCE}

Two different siRNAs were purchased from Ambion (Invitrogen, Carlsbad, CA, USA) targeting PRKCE: "select s11102" and "select s11103" (hereafter referred to as PRKCE siRNA \#2 and \#3, respectively). Ambion "select negative control siRNA \#1" was used as a negative control (hereafter referred to as "scramble"). Transfection reagents were purchased from Dharmacon Thermo Scientific (Rockford, IL, USA): "DharmaFECT 2" was used for the M112 cell line, and "DharmaFECT 4" was used for the M132 cell line. Transfections were performed in parallel with cell plating for experiments. $20 \mu \mathrm{M}$ siRNA and the appropriate transfection reagent were each diluted (1.5:100 and 1:100, respectively) in Opti-MEM medium (Invitrogen, Carlsbad, CA, USA) and left to incubate for $5 \mathrm{~min}$ at room temperature (RT). The siRNA and transfection reagents were then mixed together and incubated for $10 \mathrm{~min}$ at RT. $200 \mu \mathrm{L}$ of the mixture was then applied to wells of 12 -well plates, or $20 \mu \mathrm{L}$ was applied to wells of 96-well plates. $800 \mu \mathrm{L}$ (12-well plates) or $80 \mu \mathrm{L}$ (96-well plates) of cells at an appropriate concentration were then plated evenly in the wells. The cells were washed with phosphate-buffered saline (PBS) the following day and given fresh media.

\subsection{Scratch Assay}

Comparison of migration rate following knockdown of PRKC $\varepsilon$ in M132 cells was performed by plating $1.2 \times 10^{5}$ cells in 12 -well plates. All plates had grids drawn across them to allow repeated photographing of the same field. At least 12 fields were collected and analyzed for each sample on each day. Twenty four hours following plating, the cells were washed with PBS and given RPMI 1640 supplemented with $1 \%$ each of FBS and L-glutamine ("low serum media"). On the second day following plating, the confluent cells were "scratched" using a $200 \mu \mathrm{L}$ pipette tip. The cells were washed twice with PBS and fresh low serum media was added before immediately imaging the cells using an inverted microscope and camera. The average distance that the confluent edge of cells had travelled into the wound was measured for each time point. This experiment was performed in triplicate. 


\subsection{IGF-1 Induction Assay}

To determine if IGF-1 is able to induce the expression of PRKC $\varepsilon, 2 \times 10^{5}$ M1 12 or M132 cells were plated in 12-well plates. After two days the cells were washed twice with PBS and were given low serum RPMI media (1\% FBS, 1\% L-glutamine), with the exception of some cells which were retained in complete media as a control. The following day the remaining cells were given fresh low serum RPMI supplemented either with nothing ("no treatment" control) or with $50 \mathrm{ng} / \mathrm{mL}$ of IGF-1 (Sigma-Aldrich, St. Louis, MO, USA). After the appropriate length of incubation with IGF-1, the cells (4 wells) were washed with cold PBS, scraped, and then lysed in order to harvest cytosolic protein as described above. Trizol reagent was added to a fifth well of each sample for the extraction of RNA and evaluation of mRNA abundance as described above. This experiment was repeated in triplicate.

\section{Conclusions}

Supervised network analysis was used to discover differentially activated and organized genetic networks in expression profiles of metastatic-at-diagnosis osteosarcomas (MD-OS) compared to localized-at-diagnosis osteosarcomas (LD-OS). The PRKCE-RASGRP3-GNB2 network was found to be differentially activated among MD-OS expression profiles and among in vitro models of OS metastasis. It was found that MD-OS tumors do not express significantly higher levels of PRKC $\varepsilon$ overall (t-test $p=0.1873$ ), but they were more likely to exhibit high expression of PRKC $\varepsilon$ transcript, compared to the LD-OS tumors (five of fourteen MD-OS tumors had PRKCe expression greater than the maximum of the LD-OS tumors).

This result is consistent with the expression pattern observed in the panel of OS cell lines, where PRKC $\varepsilon$ was found to be more abundant at the RNA level in some of the in vitro models of OS metastasis. Specifically, PRKCe was more abundant in the M112 and M132 vs. Hu09, L06 and L13 cell line model, but not in the LM7 vs. SAOS2 or M8 vs. MG63 models. The heterogeneity of PRKC $\varepsilon$ expression among MD-OS tumors may indicate heterogeneous networks are aberrant in MD-OS tumors, or that the PRKCE-RASGRP3-GNB2 network may be disrupted through alterations of other genes in the network.

Despite reports by others of the involvement of PRKC $\varepsilon$ in cell migration $[89,90,96,98,118]$, knockdown of PRKC $\varepsilon$ using siRNA was not found to affect migration of highly metastatic M132 osteosarcoma cells. The effect of PRKCE on invasion of osteosarcoma cells was not investigated in this study, and may be a fruitful avenue of further research, as PRKCE is known to be involved in invasion of other cell systems in vitro, as well as to be involved in various other pro-metastatic pathways $[125,126]$. The absence of pro-metastatic effect of PRKC $\varepsilon$ protein in migration assays indicates that either the aberrant expression of the PRKCE-RASGRP3-GNB2 network may be related to a bystander effect, or that the pro-metastatic phenotype of PRKCE over-expression remains to be elucidated.

PRKCE protein expression in highly metastatic M112 cells was found to be induced by IGF-1 stimulation, and this may indicate that PRKCE is involved in IGF-1 dependent pathways. Beyond the PRKCE-RASGRP3-GNB2network, this article describes many aberrantly activated and organized networks among expression profiles of MD-OS tumors. A systematic functional screen of these 
networks, and determination of the predictive accuracy of these network expression patterns in independent datasets, would help differentiate the bystanders from the drivers of OS metastasis.

\section{Acknowledgments}

We would like to thank the following people: J. Wrana and G.D. Bader for helpful discussions, B. Fuchs and R. Muff, for correspondence regarding, and provision of, the Hu09 and derivate cell lines, E. Kleinerman for the provision of the SAOS2-P cell line, I. Taylor for provision of Dynemo software and discussions thereof, and Ali Muhammad for converting network adjacency tables to lists.

\section{References}

1. Kansara, M.; Thomas, D.M. Molecular pathogenesis of osteosarcoma. DNA Cell Biol. 2007, 26, 1-18.

2. Murphey, M.D.; Robbin, M.R.; McRae, G.A.; Flemming, D.J.; Temple, H.T.; Kransdorf, M.J. The many faces of osteosarcoma. Radiographics 1997, 17, 1205-1231.

3. PosthumaDeBoer, J.; Witlox, M.A.; Kaspers, G.J.; van Royen, B.J. Molecular alterations as target for therapy in metastatic osteosarcoma: A review of literature. Clin. Exp. Metastasis 2011, 28, 493-503.

4. Clark, J.C.; Dass, C.R.; Choong, P.F. A review of clinical and molecular prognostic factors in osteosarcoma. J. Cancer Res. Clin. Oncol. 2008, 134, 281-297.

5. Kong, C.; Hansen, M.F. Biomarkers in Osteosarcoma. Expert Opin. Med. Diagn. 2009, 3, $13-23$.

6. Schulze, A.; Downward, J. Navigating gene expression using microarrays-A technology review. Nat. Cell Biol. 2001, 3, E190-E195.

7. Dupuy, A.; Simon, R.M. Critical review of published microarray studies for cancer outcome and guidelines on statistical analysis and reporting. J. Natl. Cancer Inst. 2007, 99, 147-157.

8. Goeman, J.J.; Buhlmann, P. Analyzing gene expression data in terms of gene sets: Methodological issues. Bioinformatics 2007, 23, 980-987.

9. Song, S.; Black, M.A. Microarray-based gene set analysis: A comparison of current methods. BMC Bioinformatics 2008, 9, 502.

10. Subramanian, A.; Kuehn, H.; Gould, J.; Tamayo, P.; Mesirov, J.P. GSEA-P: A d esktop application for Gene Set Enrichment Analysis. Bioinformatics 2007, 23, 3251-3253.

11. Chuang, H.Y.; Lee, E.; Liu, Y.T.; Lee, D.; Ideker, T. Network-based classification of breast cancer metastasis. Mol. Syst. Biol. 2007, 3, 140.

12. Taylor, I.W.; Linding, R.; Warde-Farley, D.; Liu, Y.; Pesquita, C.; Faria, D.; Bull, S.; Pawson, T.; Morris, Q.; Wrana, J.L. Dynamic modularity in protein interaction networks predicts breast cancer outcome. Nat. Biotechnol. 2009, 27, 199-204.

13. Perou, C.M.; Sorlie, T.; Eisen, M.B.; van de Rijn, M.; Jeffrey, S.S.; Rees, C.A.; Pollack, J.R.; Ross, D.T.; Johnsen, H.; Akslen, L.A.; et al. Molecular portraits of human breast tumours. Nature 2000, 406, 747-752. 
14. Van’t Veer, L.J.; Dai, H.; van de Vijver, M.J.; He, Y.D.; Hart, A.A.; Mao, M.; Peterse, H.L.; van der Kooy, K.; Marton, M.J.; Witteveen, A.T.; et al. Gene expression profiling predicts clinical outcome of breast cancer. Nature 2002, 415, 530-536.

15. Cerami, E.G.; Gross, B.E.; Demir, E.; Rodchenkov, I.; Babur, O.; Anwar, N.; Schultz, N.; Bader, G.D.; Sander, C. Pathway Commons, a web resource for biological pathway data. Nucleic Acids Res. 2011, 39, D685-D690.

16. Osaka, E.; Suzuki, T.; Osaka, S.; Yoshida, Y.; Sugita, H.; Asami, S.; Tabata, K.; Hemmi, A.; Sugitani, M.; Nemoto, N.; et al. Survivin as a prognostic factor for osteosarcoma patients. Acta Histochem. Cytochem. 2006, 39, 95-100.

17. Pradelli, E.; Karimdjee-Soilihi, B.; Michiels, J.F.; Ricci, J.E.; Millet, M.A.; Vandenbos, F.; Sullivan, T.J.; Collins, T.L.; Johnson, M.G.; Medina, J.C.; et al. Antagonism of chemokine receptor CXCR3 inhibits osteosarcoma metastasis to 1 ungs. Int. J. Cancer 2009, 125, 2586-2594.

18. Cantiani, L.; Manara, M.C.; Zucchini, C.; de Sanctis, P.; Zuntini, M.; Valvassori, L.; Serra, M.; Olivero, M.; di Renzo, M.F.; Colombo, M.P.; et al. Caveolin-1 reduces osteosarcoma metastases by inhibiting c-Src activity and met signaling. Cancer Res. 2007, 67, 7675-7685.

19. Entz-Werle, N.; Marcellin, L.; Gaub, M.P.; Guerin, E.; Schneider, A.; Berard-Marec, P.; Kalifa, C.; Brugiere, L.; Pacquement, H.; Schmitt, C.; et al. Prognostic significance of allelic imbalance at the c-kit gene locus and c-kit overexpression by immunohistochemistry in pediatric osteosarcomas. J. Clin. Oncol. 2005, 23, 2248-2255.

20. Boulytcheva, I.V.; Soloviev, Y.N.; Kushlinskii, N.E.; Mahson, A.N. Expression of molecular markers in the tumor and survival prognosis in osteosarcoma. Bull. Exp. Biol. Med. 2010, $150,237-242$.

21. Oda, Y.; Wehrmann, B.; Radig, K.; Walter, H.; Rose, I.; Neumann, W.; Roessner, A. Expression of growth factors and their receptors in human osteosarcomas. Immunohistochemical detection of epidermal growth factor, platelet-derived growth factor and their receptors: Its correlation with proliferating activities and p53 expression. Gen. Diagn. Pathol. 1995, 141, 97-103.

22. Fromigue, O.; Hamidouche, Z.; Vaudin, P.; Lecanda, F.; Patino, A.; Barbry, P.; Mari, B.; Marie, P.J. CYR61 downregulation reduces osteosarcoma cell invasion, migration, and metastasis. J. Bone Miner. Res. 2011, 26, 1533-1542.

23. Hoang, B.H.; Kubo, T.; Healey, J.H.; Sowers, R.; Mazza, B.; Yang, R.; Huvos, A.G.; Meyers, P.A.; Gorlick, R. Expression of LDL receptor-related protein 5 (LRP5) as a novel marker for disease progression in high-grade osteosarcoma. Int. J. Cancer 2004, 109, 106-111.

24. Boulytcheva, I.V.; Soloviev, Y.N.; Kushlinskii, N.E.; Mahson, A.N. Expression of molecular markers in the tumor and survival prognosis in osteosarcoma. Bull. Exp. Biol. Med. 2010, 150, 237-242.

25. Ferrari, S.; Bertoni, F.; Zanella, L.; Setola, E.; Bacchini, P.; Alberghini, M.; Versari, M.; Bacci, G. Evaluation of P-glycoprotein, HER-2/ErbB-2, p53, and Bcl-2 in primary tumor and metachronous lung metastases in patients with high-grade osteosarcoma. Cancer 2004, 100, 1936-1942. 
26. Gorlick, R.; Huvos, A.G.; Heller, G.; Aledo, A.; Beardsley, G.P.; Healey, J.H.; Meyers, P.A. Expression of HER2/erbB-2 correlates with survival in osteosarcoma. J. Clin. Oncol. 1999, 17, 2781-2788.

27. Onda, M.; Matsuda, S.; Higaki, S.; Iijima, T.; Fukushima, J.; Yokokura, A.; Kojima, T.; Horiuchi, H.; Kurokawa, T.; Yamamoto, T. ErbB-2 expression is correlated with poor prognosis for patients with osteosarcoma. Cancer 1996, 77, 71-78.

28. Scotlandi, K.; Manara, M.C.; Hattinger, C.M.; Benini, S.; Perdichizzi, S.; Pasello, M.; Bacci, G.; Zanella, L.; Bertoni, F.; Picci, P.; et al. Prognostic and therapeutic relevance of HER2 expression in osteosarcoma and Ewing's sarcoma. Eur. J. Cancer 2005, 41, 1349-1361.

29. Zhou, H.; Randall, R.L.; Brothman, A.R.; Maxwell, T.; Coffin, C.M.; Goldsby, R.E. Her-2/neu expression in osteosarcoma increases risk of lung metastasis and can be associated with gene amplification. J. Pediatr. Hematol. Oncol. 2003, 25, 27-32.

30. Perbal, B.; Zuntini, M.; Zambelli, D.; Serra, M.; Sciandra, M.; Cantiani, L.; Lucarelli, E.; Picci, P.; Scotlandi, K. Prognostic value of CCN3 in osteosarcoma. Clin. Cancer Res. 2008, 14, 701-709.

31. Bjornland, K.; Flatmark, K.; Pettersen, S.; Aaasen, A.O.; Fodstad, O.; Maelandsmo, G.M. Matrix metalloproteinases participate in osteosarcoma invasion. J. Surg. Res. 2005, 127, $151-156$.

32. Gordon, N.; Koshkina, N.V.; Jia, S.F.; Khanna, C.; Mendoza, A.; Worth, L.L.; Kleinerman, E.S. Corruption of the Fas pathway delays the pulmonary clearance of murine osteosarcoma cells, enhances their metastatic potential, and reduces the effect of aerosol gemcitabine. Clin. Cancer Res. 2007, 13, 4503-4510.

33. Weber, G.F.; Bronson, R.T.; Ilagan, J.; Cantor, H.; Schmits, R.; Mak, T.W. Absence of the CD44 gene prevents sarcoma metastasis. Cancer Res. 2002, 62, 2281-2286.

34. Kubo, T.; Piperdi, S.; Rosenblum, J.; Antonescu, C.R.; Chen, W.; Kim, H.S.; Huvos, A.G.; Sowers, R.; Meyers, P.A.; Healey, J.H.; et al. Platelet-derived growth factor receptor as a prognostic marker and a therapeutic target for imatinib mesylate therapy in osteosarcoma. Cancer 2008, 112, 2119-2129.

35. Sulzbacher, I.; Birner, P.; Trieb, K.; Traxler, M.; Lang, S.; Chott, A. Expression of platelet-derived growth factor-AA is associated with tumor progression in osteosarcoma. Mod. Pathol. 2003, 16, 66-71.

36. Mizobuchi, H.; Garcia-Castellano, J.M.; Philip, S.; Healey, J.H.; Gorlick, R. Hypoxia markers in human osteosarcoma: An exploratory study. Clin. Orthop. Relat. Res. 2008, 466, 2052-2059.

37. Kashima, T.; Nakamura, K.; Kawaguchi, J.; Takanashi, M.; Ishida, T.; Aburatani, H.; Kudo, A.; Fukayama, M.; Grigoriadis, A.E. Overexpression of cadherins suppresses pulmonary metastasis of osteosarcoma in vivo. Int. J. Cancer 2003, 104, 147-154.

38. Ek, E.T.; Dass, C.R.; Contreras, K.G.; Choong, P.F. PEDF-derived synthetic peptides exhibit antitumor activity in an orthotopic model of human osteosarcoma. J. Orthop. Res. 2007, 25, $1671-1680$. 
39. Duan, X.; Jia, S.F.; Koshkina, N.; Kleinerman, E.S. Intranasal interleukin-12 gene therapy enhanced the activity of ifosfamide against osteosarcoma lung metastases. Cancer 2006, 106, $1382-1388$.

40. $\mathrm{Xu}, \mathrm{J}$.; Wu, S.; Shi, X. Expression of matrix metalloproteinase regulator, RECK, and its clinical significance in osteosarcoma. J. Orthop. Res. 2010, 28, 1621-1625.

41. Kaya, M.; Wada, T.; Nagoya, S.; Yamashita, T. Prevention of postoperative progression of pulmonary metastases in osteosarcoma by antiangiogenic therapy using endostatin. J. Orthop. Sci. 2007, 12, 562-567.

42. Luu, H.H.; Zhou, L.; Haydon, R.C.; Deyrup, A.T.; Montag, A.G.; Huo, D.; Heck, R.; Heizmann, C.W.; Peabody, T.D.; Simon, M.A.; He, T.C. Increased expression of S100A6 is associated with decreased metastasis and inhibition of cell migration and anchorage independent growth in human osteosarcoma. Cancer Lett. 2005, 229, 135-148.

43. Braczkowski, R.; Schally, A.V.; Plonowski, A.; Varga, J.L.; Groot, K.; Krupa, M.; Armatis, P. Inhibition of proliferation in human MNNG/HOS osteosarcoma and SK-ES-1 Ewing sarcoma cell lines in vitro and in vivo by antagonists of growth hormone-releasing hormone: Effects on insulin-like growth factor II. Cancer 2002, 95, 1735-1745.

44. Burrow, S.; Andrulis, I.L.; Pollak, M.; Bell, R.S. Expression of insulin-like growth factor receptor, IGF-1, and IGF-2 in primary and metastatic osteosarcoma. J. Surg. Oncol. 1998, 69, $21-27$.

45. Duan, Z.; Choy, E.; Harmon, D.; Yang, C.; Ryu, K.; Schwab, J.; Mankin, H.; Hornicek, F.J. Insulin-like growth factor-I receptor tyrosine kinase inhibitor cyclolignan picropodophyllin inhibits proliferation and induces apoptosis in multidrug resistant osteosarcoma cell lines. Mol. Cancer Ther. 2009, 8, 2122-2130.

46. Kolb, E.A.; Gorlick, R.; Houghton, P.J.; Morton, C.L.; Lock, R.; Carol, H.; Reynolds, C.P.; Maris, J.M.; Keir, S.T.; Billups, C.A.; et al. Initial testing (stage 1) of a monoclonal antibody (SCH 717454) against the IGF-1 receptor by the pediatric preclinical testing program. Pediatr. Blood Cancer 2008, 50, 1190-1197.

47. Kolb, E.A.; Kamara, D.; Zhang, W.; Lin, J.; Hingorani, P.; Baker, L.; Houghton, P.; Gorlick, R. R1507, a fully human monoclonal antibody targeting IGF-1R, is effective alone and in combination with rapamycin in inhibiting growth of osteosarcoma xenografts. Pediatr. Blood Cancer 2010, 55, 67-75.

48. Pinski, J.; Schally, A.V.; Groot, K.; Halmos, G.; Szepeshazi, K.; Zarandi, M.; Armatis, P. Inhibition of growth of human osteosarcomas by antagonists of growth hormone-releasing hormone. J. Natl. Cancer Inst. 1995, 87, 1787-1794.

49. Pinski, J.; Schally, A.V.; Halmos, G.; Szepeshazi, K.; Groot, K. Somatostatin analog RC-160 inhibits the growth of human osteosarcomas in nude mice. Int. J. Cancer 1996, 65, 870-874.

50. Pollack, R. Suramin Blockade of InsulinLike Growth Factor I-Stimulated Proliferation of Human Osteosarcoma Cells. J. Natl. Cancer Inst. 1990, 82, 1349-1352.

51. Sekyi-Otu, A.; Bell, R.S.; Ohashi, C.; Pollak, M.; Andrulis, I.L. Insulin-like growth factor 1 (IGF-1) receptors, IGF-1, and IGF-2 are expressed in primary human sarcomas. Cancer Res. 1995, 55, 129-134. 
52. Kim, S.Y.; Lee, C.H.; Midura, B.V.; Yeung, C.; Mendoza, A.; Hong, S.H.; Ren, L.; Wong, D.; Korz, W.; Merzouk, A.; et al. Inhibition of the CXCR4/CXCL12 chemokine pathway reduces the development of murine pulmonary metastases. Clin. Exp. Metastasis 2008, 25, 201-211.

53. Laverdiere, C.; Hoang, B.H.; Yang, R.; Sowers, R.; Qin, J.; Meyers, P.A.; Huvos, A.G.; Healey, J.H.; Gorlick, R. Messenger RNA expression levels of CXCR4 correlate with metastatic behavior and outcome in patients with osteosarcoma. Clin. Cancer Res. 2005, 11, 2561-2567.

54. Lin, F.; Zheng, S.E.; Shen, Z.; Tang, L.N.; Chen, P.; Sun, Y.J.; Zhao, H.; Yao, Y. Relationships between levels of CXCR4 and VEGF and blood-borne metastasis and survival in patients with osteosarcoma. Med. Oncol. 2011, 28, 649-653.

55. Oda, Y.; Yamamoto, H.; Tamiya, S.; Matsuda, S.; Tanaka, K.; Yokoyama, R.; Iwamoto, Y.; Tsuneyoshi, M. CXCR4 and VEGF expression in the primary site and the metastatic site of human osteosarcoma: Analysis within a gr oup of patients, all of whom developed lung metastasis. Mod. Pathol. 2006, 19, 738-745.

56. Dalla-Torre, C.A.; Yoshimoto, M.; Lee, C.H.; Joshua, A.M.; de Toledo, S.R.; Petrilli, A.S.; Andrade, J.A.; Chilton-MacNeill, S.; Zielenska, M.; Squire, J.A. Effects of THBS3, SPARC and SPP1 e xpression on biological behavior and survival in patients with osteosarcoma. BMC Cancer 2006, 6, 237.

57. Engin, F.; Bertin, T.; Ma, O.; Jiang, M.M.; Wang, L.; Sutton, R.E.; Donehower, L.A.; Lee, B. Notch signaling contributes to the pathogenesis of human osteosarcomas. Hum. Mol. Genet. 2009, 18, 1464-1470.

58. Ferrari, C.; Benassi, S.; Ponticelli, F.; Gamberi, G.; Ragazzini, P.; Pazzaglia, L.; Balladelli, A.; Bertoni, F.; Picci, P. Role of MMP-9 and its tissue inhibitor TIMP-1 in human osteosarcoma: Findings in 42 patients followed for 1-16 years. Acta Orthop. Scand. 2004, 75, 487-491.

59. Yang, C.; Ji, D.; Weinstein, E.J.; Choy, E.; Hornicek, F.J.; Wood, K.B.; Liu, X.; Mankin, H.; Duan, Z. The kinase Mirk is a potential therapeutic target in osteosarcoma. Carcinogenesis 2010, 31, 552-558.

60. Kersting, C.; Gebert, C.; Agelopoulos, K.; Schmidt, H.; van Diest, P.J.; Juergens, H.; Winkelmann, W.; Kevric, M.; Gosheger, G.; Brandt, B.; et al. Epidermal growth factor receptor expression in high-grade osteosarcomas is associated with a good clinical outcome. Clin. Cancer Res. 2007, 13, 2998-3005.

61. Dass, C.R.; Nadesapillai, A.P.; Robin, D.; Howard, M.L.; Fisher, J.L.; Zhou, H.; Choong, P.F. Downregulation of uPAR confirms link in growth and metastasis of osteosarcoma. Clin. Exp. Metastasis 2005, 22, 643-652.

62. Uchibori, M.; Nishida, Y.; Nagasaka, T.; Yamada, Y.; Nakanishi, K.; Ishiguro, N. Increased expression of membrane-type matrix metalloproteinase-1 is correlated with poor prognosis in patients with osteosarcoma. Int. J. Oncol. 2006, 28, 33-42.

63. Khanna, C.; Wan, X.; Bose, S.; Cassaday, R.; Olomu, O.; Mendoza, A.; Yeung, C.; Gorlick, R.; Hewitt, S.M.; Helman, L.J. The membrane-cytoskeleton linker ezrin is necessary for osteosarcoma metastasis. Nat. Med. 2004, 10, 182-186. 
64. Kim, M.S.; Song, W.S.; Cho, W.H.; Lee, S.Y.; Jeon, D.G. Ezrin expression predicts survival in stage IIB osteosarcomas. Clin. Orthop. Relat. Res. 2007, 459, 229-236.

65. Abdeen, A.; Chou, A.J.; Healey, J.H.; Khanna, C.; Osborne, T.S.; Hewitt, S.M.; Kim, M.; Wang, D.; Moody, K.; Gorlick, R. Correlation between clinical outcome and growth factor pathway expression in osteogenic sarcoma. Cancer 2009, 115, 5243-5250.

66. Charity, R.M.; Foukas, A.F.; Deshmukh, N.S.; Grimer, R.J. Vascular endothelial growth factor expression in osteosarcoma. Clin. Orthop. Relat. Res. 2006, 448, 193-198.

67. Feng, Y.; Hu, J.; Ma, J.; Feng, K.; Zhang, X.; Yang, S.; Wang, W.; Zhang, J.; Zhang, Y. RNAi-mediated silencing of VEGF-C inhibits non-small cell lung cancer progression by simultaneously down-regulating the CXCR4, CCR7, VEGFR-2 and VEGFR-3-dependent axes-induced ERK, p38 and AKT signalling pathways. Eur. J. Cancer 2011, 47, 2353-2363.

68. Kaya, M.; Wada, T.; Akatsuka, T.; Kawaguchi, S.; Nagoya, S.; Shindoh, M.; Higashino, F.; Mezawa, F.; Okada, F.; Ishii, S. Vascular endothelial growth factor expression in untreated osteosarcoma is predictive of pulmonary metastasis and poor prognosis. Clin. Cancer Res. 2000, 6, 572-577.

69. Kaya, M.; Wada, T.; Kawaguchi, S.; Nagoya, S.; Yamashita, T.; Abe, Y.; Hiraga, H.; Isu, K.; Shindoh, M.; Higashino, F.; et al. Increased pre-therapeutic serum vascular endothelial growth factor in patients with early clinical relapse of osteosarcoma. Br. J. Cancer 2002, 86, 864-869.

70. Kaya, M.; Wada, T.; Nagoya, S.; Sasaki, M.; Matsumura, T.; Yamashita, T. The level of vascular endothelial growth factor as a predictor of a poor prognosis in osteosarcoma. J. Bone Joint Surg. Br. 2009, 91, 784-788.

71. Lee, Y.H.; Tokunaga, T.; Oshika, Y.; Suto, R.; Yanagisawa, K.; Tomisawa, M.; Fukuda, H.; Nakano, H.; Abe, S.; Tateishi, A.; et al. Cell-retained isoforms of vascular endothelial growth factor (VEGF) are correlated with poor prognosis in osteosarcoma. Eur. J. Cancer 1999, 35, 1089-1093.

72. Sulzbacher, I.; Birner, P.; Trieb, K.; Lang, S.; Chott, A. Expression of osteopontin and vascular endothelial growth factor in benign and malignant bone tumors. Virchows Arch. 2002, 441, 345-349.

73. Foukas, A.F.; Deshmukh, N.S.; Grimer, R.J.; Mangham, D.C.; Mangos, E.G.; Taylor, S. Stage-IIB osteosarcomas around the knee. A study of MMP-9 in surviving tumour cells. J. Bone Joint Surg. Br. 2002, 84, 706-711.

74. Himelstein, B.P.; Asada, N.; Carlton, M.R.; Collins, M.H. Matrix metalloproteinase-9 (MMP-9) expression in childhood osseous osteosarcoma. Med. Pediatr. Oncol. 1998, 31, 471-474.

75. Gordon, N.; Arndt, C.A.; Hawkins, D.S.; Doherty, D.K.; Inwards, C.Y.; Munsell, M.F.; Stewart, J.; Koshkina, N.V.; Kleinerman, E.S. Fas expression in lung metastasis from osteosarcoma patients. J. Pediatr. Hematol. Oncol. 2005, 27, 611-615.

76. Koshkina, N.V.; Khanna, C.; Mendoza, A.; Guan, H.; DeLauter, L.; Kleinerman, E.S. Fas-negative osteosarcoma tumor cells are selected during metastasis to the lungs: The role of the Fas pathway in the metastatic process of osteosarcoma. Mol. Cancer Res. 2007, 5, 991-999. 
77. Lafleur, E.A.; Koshkina, N.V.; Stewart, J.; Jia, S.F.; Worth, L.L.; Duan, X.; Kleinerman, E.S. Increased Fas expression reduces the metastatic potential of human osteosarcoma cells. Clin Cancer Res. 2004, 10, 8114-8119.

78. Asai, T.; Tomita, Y.; Nakatsuka, S.; Hoshida, Y.; Myoui, A.; Yoshikawa, H.; Aozasa, K. VCP (p97) regulates NFkappaB signaling pathway, which is important for metastasis of osteosarcoma cell line. Jpn. J. Cancer Res. 2002, 93, 296-304.

79. Rubin, E.M.; Guo, Y.; Tu, K.; Xie, J.; Zi, X.; Hoang, B.H. Wnt inhibitory factor 1 decreases tumorigenesis and metastasis in osteosarcoma. Mol. Cancer Ther. 2010, 9, 731-741.

80. De Nigris, F.; Rossiello, R.; Schiano, C.; Arra, C.; Williams-Ignarro, S.; Barbieri, A.; Lanza, A.; Balestrieri, A.; Giuliano, M.T.; Ignarro, L.J.; et al. Deletion of Yin Yang 1 protein in osteosarcoma cells on cell invasion and CXCR4/angiogenesis and metastasis. Cancer Res. 2008, 68, 1797-1808.

81. Han, J.D.; Bertin, N.; Hao, T.; Goldberg, D.S.; Berriz, G.F.; Zhang, L.V.; Dupuy, D.; Walhout, A.J.; Cusick, M.E.; Roth, F.P.; et al. Evidence for dynamically organized modularity in the yeast protein-protein interaction network. Nature 2004, 430, 88-93.

82. Aziz, M.H.; Manoharan, H.T.; Verma, A.K. Protein kinase C epsilon, which sensitizes skin to sun's UV radiation-induced cutaneous damage and development of squamous cell carcinomas, associates with Stat3. Cancer Res. 2007, 67, 1385-1394.

83. Gundimeda, U.; Schiffman, J.E.; Gottlieb, S.N.; Roth, B.I.; Gopalakrishna, R. Negation of the cancer-preventive actions of selenium by over-expression of protein kinase Cepsilon and selenoprotein thioredoxin reductase. Carcinogenesis 2009, 30, 1553-1561.

84. Hafeez, B.B.; Zhong, W.; Weichert, J.; Dreckschmidt, N.E.; Jamal, M.S.; Verma, A.K. Genetic ablation of PKC epsilon inhibits prostate cancer development and metastasis in transgenic mouse model of prostate adenocarcinoma. Cancer Res. 2011, 71, 2318-2327.

85. McJilton, M.A.; van Sikes, C.; Wescott, G.G.; Wu, D.; Foreman, T.L.; Gregory, C.W.; Weidner, D.A.; Harris Ford, O.; Morgan Lasater, A.; Mohler, J.L.; et al. Protein kinase Cepsilon interacts with Bax and promotes survival of human prostate cancer cells. Oncogene 2003, 22, 7958-7968.

86. Meshki, J.; Caino, M.C.; von Burstin, V.A.; Griner, E.; Kazanietz, M.G. Regulation of prostate cancer cell survival by protein kinase Cepsilon involves bad phosphorylation and modulation of the TNFalpha/JNK pathway. J. Biol. Chem. 2010, 285, 26033-26040.

87. Wu, D.; Foreman, T.L.; Gregory, C.W.; McJilton, M.A.; Wescott, G.G.; Ford, O.H.; Alvey, R.F.; Mohler, J.L.; Terrian, D.M. Protein kinase cepsilon has the potential to advance the recurrence of human prostate cancer. Cancer Res. 2002, 62, 2423-2429.

88. Grossoni, V.C.; Todaro, L.B.; Kazanietz, M.G.; de Kier Joffe, E.D.; Urtreger, A.J. Opposite effects of protein kinase $\mathrm{C}$ beta1 (PKCbeta1) and PKCepsilon in the metastatic potential of a breast cancer murine model. Breast Cancer Res. Treat. 2009, 118, 469-480.

89. Pan, Q.; Bao, L.W.; Kleer, C.G.; Sabel, M.S.; Griffith, K.A.; Teknos, T.N.; Merajver, S.D. Protein kinase $\mathrm{C}$ epsilon is a predictive biomarker of aggressive breast cancer and a validated target for RNA interference anticancer therapy. Cancer Res. 2005, 65, 8366-8371. 
90. Huang, B.; Cao, K.; Li, X.; Guo, S.; Mao, X.; Wang, Z.; Zhuang, J.; Pan, J.; Mo, C.; Chen, J.; et al. The expression and role of protein kinase $\mathrm{C}(\mathrm{PKC})$ epsilon in clear cell renal cell carcinoma. J. Exp. Clin. Cancer Res. 2011, 30, 88.

91. Jansen, A.P.; Verwiebe, E.G.; Dreckschmidt, N.E.; Wheeler, D.L.; Oberley, T.D.; Verma, A.K. Protein kinase C-epsilon transgenic mice: A unique model for metastatic squamous cell carcinoma. Cancer Res. 2001, 61, 808-812.

92. Reddig, P.J.; Dreckschmidt, N.E.; Zou, J.; Bourguignon, S.E.; Oberley, T.D.; Verma, A.K. Transgenic mice overexpressing protein kinase $\mathrm{C}$ epsilon in their epidermis exhibit reduced papilloma burden but enhanced carcinoma formation after tumor promotion. Cancer Res. 2000, 60, 595-602.

93. Sand, J.M.; Aziz, M.H.; Dreckschmidt, N.E.; Havighurst, T.C.; Kim, K.; Oberley, T.D.; Verma, A.K. PKCepsilon overexpression, irrespective of genetic background, sensitizes skin to UVR-induced development of squamous-cell carcinomas. J. Investig. Dermatol. 2010, 130, 270-277.

94. Sand, J.M.; Hafeez, B.B.; Aziz, M.H.; Siebers, E.M.; Dreckschmidt, N.E.; Verma, A.K. Ultraviolet radiation and 12-O-tetradecanoylphorbol-13-acetate-induced interaction of mouse epidermal protein kinase $\mathrm{C}$ epsilon with Stat3 involve integration with Erk1/2. Mol. Carcinog. 2012, 51, 291-302.

95. Wheeler, D.L.; Ness, K.J.; Oberley, T.D.; Verma, A.K. Inhibition of the development of metastatic squamous cell carcinoma in protein kinase $\mathrm{C}$ epsilon transgenic mice by alpha-difluoromethylornithine accompanied by marked hair follicle degeneration and hair loss. Cancer Res. 2003, 63, 3037-3042.

96. Bao, L.; Gorin, M.A.; Zhang, M.; Ventura, A.C.; Pomerantz, W.C.; Merajver, S.D.; Teknos, T.N.; Mapp, A.K.; Pan, Q. Preclinical development of a bifunctional cancer cell homing, PKCepsilon inhibitory peptide for the treatment of head and neck cancer. Cancer Res. 2009, 69, 5829-5834.

97. Martinez-Gimeno, C.; Diaz-Meco, M.T.; Dominguez, I.; Moscat, J. Alterations in levels of different protein kinase $\mathrm{C}$ isotypes and their influence on behavior of squamous cell carcinoma of the oral cavity: Epsilon PKC, a novel prognostic factor for relapse and survival. Head Neck 1995, 17, 516-525.

98. Pan, Q.; Bao, L.W.; Teknos, T.N.; Merajver, S.D. Targeted disruption of protein kinase C epsilon reduces cell invasion and motility through inactivation of RhoA and RhoC GTPases in head and neck squamous cell carcinoma. Cancer Res. 2006, 66, 9379-9384.

99. Tosi, L.; Rinaldi, E.; Carinci, F.; Farina, A.; Pastore, A.; Pelucchi, S.; Cassano, L.; Evangelisti, R.; Carinci, P.; Volinia, S. Akt, protein kinase C, and mitogen-activated protein kinase phosphorylation status in head and neck squamous cell carcinoma. Head Neck 2005, 27, 130-137.

100. Bae, K.M.; Wang, H.; Jiang, G.; Chen, M.G.; Lu, L.; Xiao, L. Protein kinase C epsilon is overexpressed in primary human non-small cell lung cancers and functionally required for proliferation of non-small cell lung cancer cells in a p21/Cip1-dependent manner. Cancer Res. 2007, 67, 6053-6063. 
101. Caino, M.C.; von Burstin, V.A.; Lopez-Haber, C.; Kazanietz, M.G. Differential regulation of gene expression by protein kinase $\mathrm{C}$ isozymes as determined by genome-wide expression analysis. J. Biol. Chem. 2011, 286, 11254-11264.

102. Ding, L.; Wang, H.; Lang, W.; Xiao, L. Protein kinase C-epsilon promotes survival of lung cancer cells by suppressing apoptosis through dysregulation of the mitochondrial caspase pathway. J. Biol. Chem. 2002, 277, 35305-35313.

103. Felber, M.; Sonnemann, J.; Beck, J.F. Inhibition of novel protein kinase $C$-epsilon augments TRAIL-induced cell death in A549 lung cancer cells. Pathol. Oncol. Res. 2007, 13, 295-301.

104. Aviel-Ronen, S.; Coe, B.P.; Lau, S.K.; da Cunha Santos, G.; Zhu, C.Q.; Strumpf, D.; Jurisica, I.; Lam, W.L.; Tsao, M.S. Genomic markers for malignant progression in pulmonary adenocarcinoma with bronchioloalveolar features. Proc. Natl. Acad. Sci. USA 2008, 105, 10155-10160.

105. Yang, D.; Kedei, N.; Li, L.; Tao, J.; Velasquez, J.F.; Michalowski, A.M.; Toth, B.I.; Marincsak, R.; Varga, A.; Biro, T.; et al. RasGRP3 contributes to formation and maintenance of the prostate cancer phenotype. Cancer Res. 2010, 70, 7905-7917.

106. Kimura, K.; Nakano, T.; Park, Y.B.; Tani, M.; Tsuda, H.; Beppu, Y.; Moriya, H.; Yokota, J. Establishment of human osteosarcoma cell lines with high metastatic potential to lungs and their utilities for therapeutic studies on metastatic osteosarcoma. Clin. Exp. Metastasis 2002, $19,477-485$.

107. Nakano, T.; Tani, M.; Ishibashi, Y.; Kimura, K.; Park, Y.B.; Imaizumi, N.; Tsuda, H.; Aoyagi, K.; Sasaki, H.; Ohwada, S.; et al. Biological properties and gene expression associated with metastatic potential of human osteosarcoma. Clin. Exp. Metastasis 2003, 20, 665-674.

108. Jia, S.F.; Worth, L.L.; Kleinerman, E.S. A nude mouse model of human osteosarcoma lung metastases for evaluating new therapeutic strategies. Clin. Exp. Metastasis 1999, 17, 501-506.

109. Zhu, W.; Guo, F.; Xu, T.; Chen, A. Establishment of nude mice model of human osteosarcoma cell line MG63 with different potential of metastasis. Chin. Ger. J. Clin. Oncol. 2007, 6, 484-486.

110. England, K.; Ashford, D.; Kidd, D.; Rumsby, M. PKC epsilon is associated with myosin IIA and actin in fibroblasts. Cell. Signal. 2002, 14, 529-536.

111. Allen, T.R.; Krueger, K.D.; Hunter, K.W.; Agrawal, D.K. Evidence that insulin-like growth factor-1 requires protein kinase $\mathrm{C}$-(epsilon), PI3-kinase and mitogen-activated protein kinase pathways to protect human vascular smooth muscle cells from apoptosis. Immunol. Cell. Biol. 2005, 83, 651-667.

112. Thommes, K.B.; Hoppe, J.; Vetter, H.; Sachinidis, A. The synergistic effect of PDGF-AA and IGF-1 on VSMC proliferation might be explained by the differential activation of their intracellular signaling pathways. Exp. Cell Res. 1996, 226, 59-66.

113. Yano, K.; Bauchat, J.R.; Liimatta, M.B.; Clemmons, D.R.; Duan, C. Down-regulation of protein kinase $\mathrm{C}$ inhibits insulin-like growth factor I-induced vascular smooth muscle cell proliferation, migration, and gene expression. Endocrinology 1999, 140, 4622-4632.

114. Newton, A.C. Regulation of the ABC kinases by phosphorylation: Protein kinase $\mathrm{C}$ as a paradigm. Biochem. J. 2003, 370, 361-371. 
115. Mamane, Y.; Petroulakis, E.; LeBacquer, O.; Sonenberg, N. mTOR, translation initiation and cancer. Oncogene 2006, 25, 6416-6422.

116. Hua, Y.; Qiu, Y.; Zhao, A.; Wang, X.; Chen, T.; Zhang, Z.; Chi, Y.; Li, Q.; Sun, W.; Li, G.; et al. Dynamic metabolic transformation in tumor invasion and metastasis in mice with LM-8 osteosarcoma cell transplantation. J. Proteome Res. 2011, 10, 3513-3521.

117. Huang, B.; Cao, K.; Li, X.; Guo, S.; Mao, X.; Wang, Z.; Zhuang, J.; Pan, J.; Mo, C.; Chen, J.; et al. The expression and role of protein kinase $\mathrm{C}$ (PKC) epsilon in clear cell renal cell carcinoma. J. Exp. Clin. Cancer Res. 2011, 30, 88.

118. Leask, A.; Shi-Wen, X.; Khan, K.; Chen, Y.; Holmes, A.; Eastwood, M.; Denton, C.P.; Black, C.M.; Abraham, D.J. Loss of protein kinase Cepsilon results in impaired cutaneous wound closure and myofibroblast function. J. Cell. Sci. 2008, 121, 3459-3467.

119. Simon, R.M.; Lam, A.; Li, M.C.; Ngan, M.; Menenzes, S.; Zhao, Y. Analysis of Gene Expression Data Using BRB-Array Tools. Cancer Inform. 2007, 3, 11-17.

120. Merico, D.; Isserlin, R.; Stueker, O.; Emili, A.; Bader, G.D. Enrichment map: A network-based method for gene-set enrichment visualization and interpretation. PLoS One 2010, 5, e13984.

121. Cline, M.S.; Smoot, M.; Cerami, E.; Kuchinsky, A.; Landys, N.; Workman, C.; Christmas, R.; Avila-Campilo, I.; Creech, M.; Gross, B.; et al. Integration of biological networks and gene expression data using Cytoscape. Nat. Protoc. 2007, 2, 2366-2382.

122. Harris, M.A.; Clark, J.; Ireland, A.; Lomax, J.; Ashburner, M.; Foulger, R.; Eilbeck, K.; Lewis, S.; Marshall, B.; Mungall, C.; et al. The Gene Ontology (GO) database and informatics resource. Nucleic Acids Res. 2004, 32, D258-D261.

123. Boyle, E.I.; Weng, S.; Gollub, J.; Jin, H.; Botstein, D.; Cherry, J.M.; Sherlock, G. GO::TermFinder-open source software for accessing Gene Ontology information and finding significantly enriched Gene Ontology terms associated with a list of genes. Bioinformatics 2004, 20, 3710-3715.

124. Eppert, K.; Wunder, J.S.; Aneliunas, V.; Kandel, R.; Andrulis, I.L. von Willebrand factor expression in osteosarcoma metastasis. Mod. Pathol. 2005, 18, 388-397.

125. Gorin, M.A.; Pan, Q. Protein kinase C epsilon: An oncogene and emerging tumor biomarker. Mol. Cancer 2009, 8, 9.

126. Griner, E.M.; Kazanietz, M.G. Protein kinase C and other diacylglycerol effectors in cancer. Nat. Rev. Cancer 2007, 7, 281-294. 
Reprinted from Cancers. Cite as Sadri, N.; Zhang, P.J. Hypoxia-Inducible Factors: Mediators of Cancer Progression; Prognostic and Therapeutic Targets in Soft Tissue Sarcomas. Cancers 2013, 5, 320-333.

Review

\title{
Hypoxia-Inducible Factors: Mediators of Cancer Progression; Prognostic and Therapeutic Targets in Soft Tissue Sarcomas
}

\author{
Navid Sadri and Paul J. Zhang *
}

Anatomic Pathology, Department of Pathology and Laboratory Medicine, Hospital of the University of Pennsylvania, 3400 Spruce Street, 6th Floor Founders Building, Philadelphia, PA 19104, USA;

E-Mail: navid.sadri@uphs.upenn.edu

* Author to whom correspondence should be addressed; E-Mail: pjz@mail.med.upenn.edu; Tel.: +1-215-662-6526; Fax: +1-215-662-3238.

Received: 23 January 2013; in revised form: 12 March 2013 / Accepted: 26 March 2013 /

Published: 2 April 2013

\begin{abstract}
Soft-tissue sarcomas remain aggressive tumors that result in death in greater than a third of patients due to either loco-regional recurrence or distant metastasis. Surgical resection remains the main choice of treatment for soft tissue sarcomas with pre- and/or post-operational radiation and neoadjuvant chemotherapy employed in more advanced stage disease. However, in recent decades, there has been little progress in the average five-year survival for the majority of patients with high-grade soft tissue sarcomas, highlighting the need for improved targeted therapeutic agents. Clinical and preclinical studies demonstrate that tumor hypoxia and up-regulation of hypoxia-inducible factors (HIFs) is associated with decreased survival, increased metastasis, and resistance to therapy in soft tissue sarcomas. HIF-mediated gene expression regulates many critical aspects of tumor biology, including cell survival, metabolic programming, angiogenesis, metastasis, and therapy resistance. In this review, we discuss HIFs and HIF-mediated genes as potential prognostic markers and therapeutic targets in sarcomas. Many pharmacological agents targeting hypoxia-related pathways are in development that may hold therapeutic potential for treating both primary and metastatic sarcomas that demonstrate increased HIF expression.
\end{abstract}

Keywords: soft tissue sarcoma; hypoxia; hypoxia-inducible factor; HIFs 


\section{Introduction}

Hypoxia is a common feature of many solid tumors owing to aberrant vascular function and rapid cell division. Neoplastic cells can survive under conditions of low oxygenation by activating adaptive responses to match oxygen supply with metabolic, bioenergetic, and redox demands [1]. Clinical and experimental studies have long demonstrated that tumor hypoxia is associated with increased malignancy, poor prognosis, and resistance to radiation and chemotherapy [2-4]. Patients with the most hypoxic soft tissue sarcomas have a worse disease-specific and overall survival [4] and increased likelihood of metastasis [5].

\subsection{Hypoxia-Inducible Factors}

The hypoxia-inducible factor (HIF) transcription factors mediate the primary transcriptional response to hypoxic stress in normal and neoplastic cells [6]. HIFs form heterodimeric complexes composed of an oxygen-liable $\alpha$ subunit and a stable $\beta$ subunit. Together these subunits bind hypoxia response elements (HREs) on several hundred genes that facilitate the adaptation to hypoxia [6,7]. Mammals possess three isoforms of HIF $\alpha$, of which HIF1 $\alpha$ and HIF2 $\alpha$ are the best characterized. HIF3 $\alpha$ lacks the $C$-terminal transactivation domain and is believed to be a negative regulator of hypoxia-inducible gene expression, most likely by competing for binding with HIF1 $\alpha$ and HIF2 $\alpha$ in conditions where HIF $\beta$ is limited [8]. HIF1 $\alpha$ is ubiquitously expressed, whereas HIF2 $\alpha$ and HIF3 $\alpha$ are selectively expressed in certain tissue types including vascular endothelium, liver parenchyma, and cells of myeloid lineage [2].

HIF activity is controlled primarily through the stabilization of HIF $1 \alpha$ and HIF2 $\alpha$ protein subunits, which increases as cells become more hypoxic. HIF $\alpha$ subunits are modified by hydroxylation of two proline residues by HIF-specific prolyl-hydroxylases (PDHs) in the presence of oxygen, which leads to normoxic proteasomal degradation that is in part mediated by the von Hippel-Lindau (VHL) tumor suppressor protein [6] (Figure 1). Furthermore, under normoxic conditions, HIF1 $\alpha$ is hydroxylated at residue Asn803 by factor inhibiting HIF1 (FIH), which disrupts a critical interaction between HIF $\alpha$ and coactivator p300, blocking HIF1-dependent transcriptional activition [1]. In the context of increased oncogenic signaling, HIF1 $\alpha$ expression is also increased in cancer cells by hypoxia-independent mechanisms that include increased transcription and/or translation of HIF1 $\alpha$ mRNA, as well as, increased protein stability [1]. Activated phosphoinositide-3 kinase (PI3K)-AKT-mammalian target of rapamycin (mTOR) signaling, a common feature of many cancer cells, increases the rate of HIF mRNA translation, leading to increased HIF expression and activity [9].

\subsection{HIFs and Soft Tissue Sarcoma}

Each year, soft tissue sarcomas arise in over 12,000 persons in the United States, and 35\% of patients die of either loco-regional recurrence or distant metastasis [10]. Like other solid tumors, as sarcomas outgrow their blood supply, hypoxia stabilizes HIF1 $\alpha$ and HIF $2 \alpha$, which bind to HIF - $\beta$ (ARNT), and drive the transcription of over 150 genes crucial in many aspects of cancer biology including angiogenesis, epithelial-mesenchymal transition, stem-cell maintenance, invasion, 
metastasis, and resistance to radiation therapy and chemotherapy [11] (Figure 1). Based on genome-wide chromatin immunoprecipitation studies the estimated number of direct HIF target genes is greater than 800 genes [8]. HIFs also indirectly regulate gene expression by transactivation of chromatin-modifying genes and microRNAs [7,12,13]. Hierarchical clustering analysis using expression data from $>100$ hypoxia-related genes on oligonucleotide microarrays of sarcomas and normal tissues identified distinctly different patterns of expression; numerous hypoxia-related genes were significantly up-regulated in sarcomas including HIF1 $\alpha$ [14].

Figure 1. HIFs are involved in crucial aspects of tumor progression and metastasis in sarcomas. In the presence of oxygen, prolyl-hydroxylases (PDH) hydroxylate proline residues on HIF $\alpha$ subunit. Hydoxylated HIF $\alpha$ interacts with von Hippel-Lindau (VHL) protein, which is part of the E3 ubiquitin ligase complex that mediates the ubiquitination (Ub) of HIF $\alpha$ and targets it for proteasomal degradation. Under hypoxia, the $\alpha$ subunit is not hydroxylated. The stabilized $\alpha$ subunit moves into the nucleus, dimerizes with $\beta$ subunit, and binds to hypoxia response elements (HRE) on target genes. HIF $\alpha$ is additionally up-regulated in a hypoxia-independent mechanism by several oncogenic pathways, including the PI3K-AKT-mTOR pathway. A representative HIF-regulated gene is shown to illustrate the importance of HIF target genes in many aspects of cancer biology in sarcomas. HIF-target genes are involved in: metabolic reprogramming, which include glucose transporter 1 (GLUT-1); induction of angiogenesis mediated by vascular endothelial growth factor (VEGF); promoting cell survival by encoding insulin-like growth factor 2 (IGF2); enhance stem-cell self-renewal ability through expression of delta-like 1 (DLK-1); promote metastasis through regulation of extracellular matrix genes, such as collagen, type $\mathrm{V}, \alpha 1$ (COL5A1); and chemotherapy resistance through expression of ATP-binding cassette transporter B1 (ABCB1) that efflux chemotherapy drugs from cancer cells. In addition, HIFs indirectly regulate gene and protein expression by transactivation of chromatin-modifying genes and microRNAs. As an example, HIF1 $\alpha$ up-regulates EWS-FL1 onco-protein expression and modulates its transcriptional signature towards metastasis-related genes.

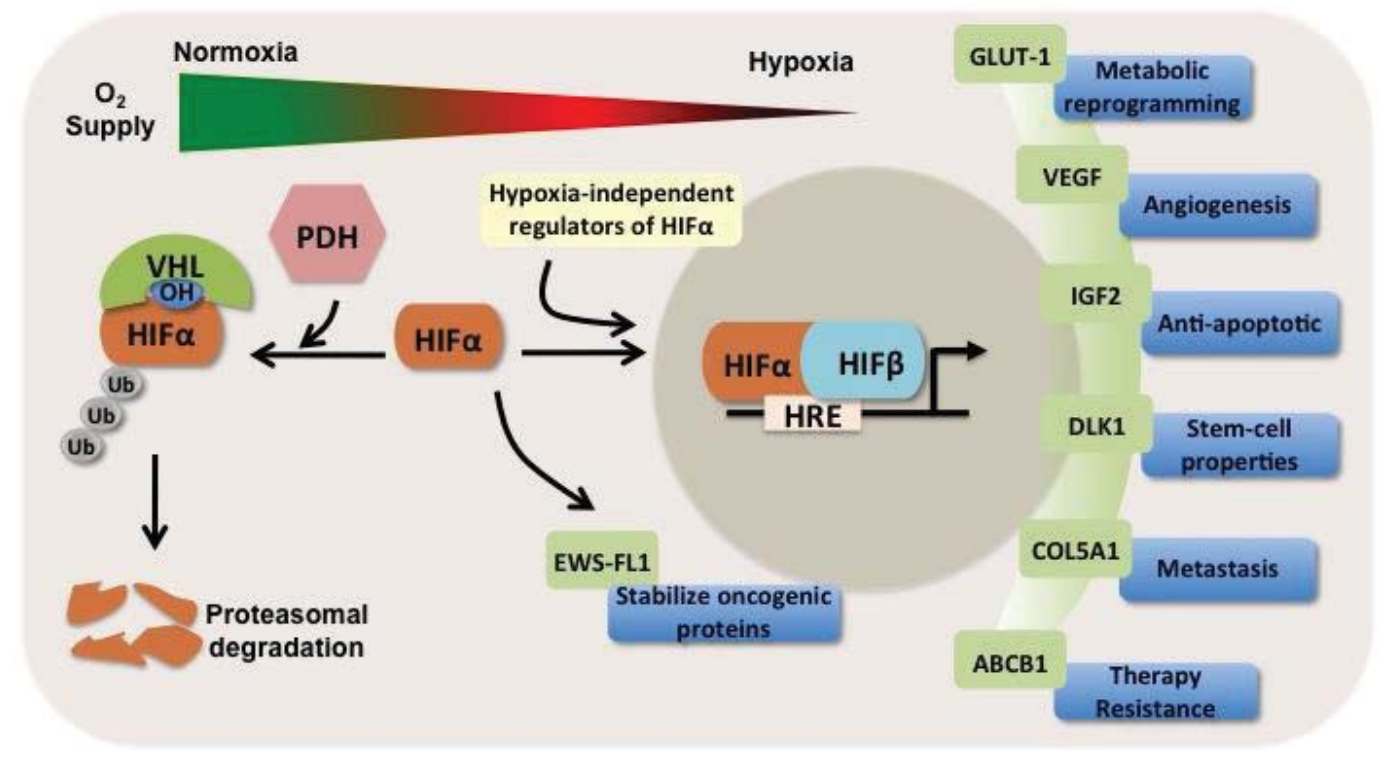


The importance of hypoxia in many human cancers has prompted intensive research into understanding the mechanisms by which hypoxic tumor cells alter their transcriptional profiles to modulate critical pathways important in cancer biology $[1,6]$. There is also a growing body of preclinical evidence supporting the importance of the HIF pathway in sarcoma progression, metastasis, and therapy resistance. Only recently has the use of HIFs and HIF-targets as prognostic markers and potential therapeutic targets in soft tissue sarcomas been explored.

\subsection{HIFs as Independent Prognosticators in Soft Tissue and Bone Sarcomas}

There is compelling evidence that activation of the HIF pathway promotes oncogenesis and cancer progression, including clinical data showing an association between increased HIF expression and decreased patient survival in many human cancers, including sarcomas [15-21]. Several reports have shown that hypoxia and elevated HIFs expression are correlated with a significantly shorter overall survival and metastasis free survival in soft tissue sarcomas, and in certain studies irrespective of histological diagnosis [4,22-25]. As an example, increased expression of HIF $1 \alpha$ and HIF $2 \alpha$ in chondrosarcomas is associated with worse patient survival $[18,19]$. HIF $1 \alpha$ and HIF2 $\alpha$ up-regulation play a prominent role in evasion of apoptosis and tumor progression associated with high Bcl-xL and low Beclin 1 expression in chondrosarcomas [18,19]. Whereas the trend from these studies is that HIF1 $\alpha$ and HIF2 $\alpha$ promote cancer progression, the association is not absolute. In one report, high expression of HIF $1 \alpha$ mRNA in certain sarcomas was correlated with a significantly more favorable prognosis [26]. This may suggest differences in transcriptional and post-transcriptional mechanisms of HIF mRNA and/or may highlight complex and even opposing function by different iso forms of HIF during different stages of tumor development.

\section{HIFs: Mediators of Sarcoma Progression, Metastasis, and Therapy Resistance}

\subsection{HIFs as Regulators of Sarcomagenesis}

Early data showing that HIF1 $\alpha$ expression was correlated with aberrant p53 accumulation and cell proliferation in various solid tumors and their metastasis indicated the important role that HIFs may play in human cancer progression [27]. Although the pathogenesis of sarcoma subtypes varies greatly, HIF-regulated genes have been shown to be important in the pathobiology of various sarcoma subtypes.

\subsubsection{Rhabdomyosarocoma}

Several lines of evidence suggest a role HIF1 $\alpha$ in rhabdomyosarcoma tumorigenesis. Under hypoxia, glucose transporter 1 (GLUT-1) is induced in a HIF1 $\alpha$-dependent manner increasing glucose uptake and playing an important role in conferring apoptosis resistance [28]. Hypoxia also enhances the stem-like population of cells within rhabdomyosarcoma cell lines [29]. More recently, pharmacological targeting of the HIF1 $\alpha$ signaling pathway has been shown to inhibit rhabdomyosarcoma growth in xenograft models [30]. 


\subsubsection{Kaposi’s Sarcoma}

As the master regulator of the hypoxic vascular response, it should not be surprising that HIFs plays a central role in Kaposi's sarcomagenesis (KS) [31]. HIF1 $\alpha$ drives transcriptional activation of hundreds of genes involved in vascular reactivity, angiogenesis, arteriogenesis, and the recruitment of endothelial precursor cells, all key steps toward the development of KS [31,32]. The latency-associated nuclear antigen (LANA) encoded by Kaposi's sarcoma-associated herpesvirus (KSHV) is critical for nuclear accumulation of HIF1 $\alpha$ in normoxia as it targets the HIF1 $\alpha$ suppressors von Hippel-Lindau protein and p53 for degradation [33,34]. KSHV G protein-coupled receptor (vGPCR) upregulates vascular endothelial growth factor (VEGF) in endothelial cells through an mTOR-dependent increase in HIF1 $\alpha$ and HIF2 $\alpha$ protein levels [32]. Pharmacologic inhibition of HIFs blocked VEGF secretion and lead to regression of tumor allografts in this model [32].

\subsubsection{Gastroinstestinal Tumor}

In a subset of "wild-type" gastrointestinal tumors (GISTs) with succinate dehydrogenase loss-of-function, it is postulated that elevation of succinate levels negatively regulates prolyl hydroxylase leading to increased HIF1 $\alpha$ levels [35]. In line with this model, wild-type GISTs show increased expression of VEGF and insulin-like growth factor 2 (IGF2), HIF1 $\alpha$ transcriptional targets, as compared to KIT-mutated GISTs [36]. IGF2 may activate IGFR in an autocrine manner resulting in increased signaling through the PI3K-AKT pathway [35].

\subsubsection{Liposarcoma}

Several lines of evidence suggest HIF1 $\alpha$ involvement in the progression of liposarcoma to a dedifferentiated state. In synchronous liposarcoma lesions that contain both well-differentiated component (adipocyte-like differentiation) and dedifferentiated component (lacking adipocyte differentiation and frequently showing other mesenchchymal differentiation), HIF $1 \alpha$ is primarily detected in the dedifferentiated component [24]. Hypoxia has been shown to inhibit adipocyte differentiation through the repressive activity of HIF1 $\alpha$-induced differentiated embryo-chondrocyte expressed gene 1 (DEC1) on PPAR 2 expression [37]. Furthermore, hypoxia via an HIF-dependent mechanism promotes the maintained expression of delta-like 1 (DLK1), a key stem cell gene that negatively regulates adipogenic differentiation and may facilitate the maintenance and/or selection of cancer cells with stem cell properties [38,39].

\subsubsection{Ewing's Sarcoma}

In Ewing's sarcoma cells, EWS-FLI1 protein expression is upregulated by hypoxia in a HIF1 $\alpha$-dependent manner [40]. Furthermore, hypoxia modulates the EWS-FLI1 transcriptional signature towards the expression of metastasis-related genes and leads to invasiveness and soft agar colony formation in vitro [40]. Although HIF1 $\alpha$ and HIF2 $\alpha$ were previously suspected to have overlapping functions, more recent data suggest isoform-specific transcriptional responses. Experiments in Ewing's sarcoma and osteosarcoma cell lines highlight isoform-specific HIF transcriptional response to hypoxia and hypoglycemia [41]. Downstream transcription of transcripts 
containing the VEGF and GLUT-1 hypoxia-response element (HRE) was HIF1 $\alpha$-dependent in Ewing's sarcoma, but regulated by both isoforms in osteosarcoma [41]. The specific mechanism(s) whereby HIF promotes Ewing's sarcoma and osteosarcoma progression in vivo remains to be identified. Furthermore, future studies need to better define isoform-specific transcriptional responses and function in an oncogenic context.

In certain contexts, hypoxia alone may not be enough to activate the HIF system. Despite the presence of extreme hypoxia, HIF1 $\alpha$ is not up-regulated in benign uterine leiomyomas, however in their malignant counterparts, leiomyosarcomas, show a strong induction of the HIF system [42]. The authors suggest the strong activation of the HIF system observed in solid malignant tumors may be mechanistically linked to their transformed phenotype, rather than being a physiological reaction activated in a pathological context [42].

\subsection{HIFs as Regulators of Metastasis}

It has been shown that tumor oxygenation predicts the likelihood of distant metastases in human sarcomas $[5,43,44]$. Gene expression data from human tumors and work with experimental mouse models highlight the importance of HIF pathway activation in sarcoma metastasis. In a genetically engineered, temporally and spatially restricted, mouse model of pleomorhpic undifferentiated sarcomas, the HIF-target FOXM1 is highly associated with lung metastasis [44]. Gene expression microarray analysis in a gr oup of $177 \mathrm{~s}$ arcomas revealed a prognostic profile of hypoxia-related genes predictive of metastatic potential in high grade, pleomorphic, genetically-complex sarcomas [43]. A separate gene expression microarray analysis suggested the existence of at least two subsets of high-grade pleomorphic STS with distinct clinical behavior, with tumors with increased metastatic propensity showing increased expression of HIF-dependent extracellular matrix genes, including COL5A1, COL1A2, and PLOD2 [45]. Independent of currently used prognosticators, these results support that hypoxia-related gene expression signature provide diagnostic utility in improved selection of high-risk STS patients.

Studies from other cancer types suggest that metastasis is achieved through a stepwise selection process driven by hypoxia [46]. HIF1 $\alpha$-dependent up-regulation of cathepsin D, urokinase-type plasminogen-activator receptor, and matrix metalloproteinase- 2 enable cellular invasion through the basement membrane and the underlying stroma [47]. Studies in breast and head and neck cancers have shown that hypoxia-induced lysyl oxidase (LOX) is essential for tumor metastasis as LOX covalently modifies collagens to increase focal adhesion kinase activity, cell migration, and metastasis [48]. Hypoxia-induced VEGF promotes intravasation and extravasation by helping to increase microvascular permeability and interstitial fluid pressure [46]. ANGPTL4, a key molecule for extravasation to the lung, is up-regulated by HIF1 $\alpha$ [49]. Hypoxia may increase metastatic homing by inducing chemokine receptor CXCR4, which plays a key role in metastatic homing of tumor cells to organs expressing high level of its ligand, SDF1 [50]. LOX also acts as a critical mobilizing factor, which recruits $\mathrm{CD} 11 \mathrm{~b}+$ myeloid cells to form the niche to facilitate the colonization of metastatic tumor cells [51]. Through regulation of these critical molecular targets, HIFs promote various steps of the metastatic cascade and provide an adaptive advantage to select tumor cell populations to survive and escape the unfavorable microenvironment of the primary 
tumor [46]. It is currently unclear if sarcoma cancer cells use similar molecular mechanisms or employ other HIF-dependent pathways to achieve metastatic potential.

Hypoxia may also influence organ-specific metastasis. In breast cancer, hypoxia enhances the expression of a large percentage of genes involved in lung metastasis, while it activates a more limited number in bo ne metastasis [27]. The role of HIFs in organ-specific metastasis needs to be better studied in sarcomas, especially with respect to lung metastasis that constitute the majority of metastatic diseases. Future studies need to address if HIF-targets that promote survival, metabolic reprogramming, invasion and angiogenesis in the primary tumor function similarly in secondary sites.

\subsection{HIFs as Regulators of Therapy Resistance}

It is well established that hypoxic cells within a tumor limit the effectiveness of radiotherapy, with the requirement of free oxygen to covert free radicals initiated by ionizing radiation to form DNA strand breaks [52]. HIFs also contribute to radiation resistance. Inhibition of HIF-2 $\alpha$ leads to tumor cell death and enhances the response to radiation treatment [53]. HIF1 $\alpha$ also promotes tumor radioresistance through stimulation of endothelial cell survival [54]. In a phase II clinical trial, neoadjuvant bevacizumab, an anti-VEGF therapy, can significantly augment the therapeutic efficacy of radiation therapy against soft tissue sarcomas and may reduce the incidence of local recurrence [55].

A contribution of HIF1 $\alpha$ to chemoresistance of neoplastic cells has been observed in a wide spectrum of solid tumors, including fibrosarcoma [56-58]. Clinically, HIF1 $\alpha$ has been shown to be an independent factor for resistance to chemotherapy in several solid tumor types [59-61]. The mechanisms underpinning HIF1 $\alpha$-mediated chemoresistance need to be better studied in the heterogenous group of sarcomas that exhibit resistance to therapy. Furthermore, HIF1 activity may contribute to resistance to $\mathrm{m}$ ore targeted therapies, such as imatinib, through metabolic reprogramming [62].

\section{Agents Targeting HIFs}

Several approaches targeting hypoxia in sarcoma cells have been explored, including the use of bioreductive prodrugs that are converted to cytotoxins under hypoxic conditions, developing inhibitors of HIF1 expression and activity, targeting oncogenic pathways (mTOR) regulating HIF1 expression, and targeting specific HIF downstream pathways [63]. Most of these agents have previously been reviewed elsewhere [11,63-65], and will not be extensively covered here.

Bioactive prodrugs, agents that only become activated in oxygen-poor conditions, are designed to exploit the hypoxic microenvironment in many sarcomas. TH-302, a nitroimidazole prodrug of the DNA alkylating agent, bromoisophosphoramide mustard (Br-IPM), is reduced in hypoxic conditions leading to release of the Br-IPM and DNA cross-linking [66]. TH-302 has minimal cytotoxic activity under normoxic conditions, minimizing systemic toxicity seen with ifosfamide, a similar systemic cross-linking agent. The standard single-agent chemotherapy for metastatic or locally advanced unresectable disease is doxorubicin, with an expected response rate of $12 \%-23 \%$ and median progression-free survival of 4-6 months [66]. Updated results of phase 2 clinical trial involving 
91 metastatic or locally advanced unresectable STS reported at 2012 Connective Tissue Oncology Society meeting in Prague showed that TH-302 boosted the response rate to $36 \%$ with a median progression-free survival of 6.7 months [66,67]. In March 2012, TH-302 was granted orphan drug status in the United States and E urope to treat soft-tissue sarcoma [68]. Given the marked heterogeneity in tumor hypoxia, there is a great need for the development of diagnostics to better predict patient stratification and sensitivity as new drugs are discovered [63].

Agents targeting HIFs are in various stages of clinical development [69]. Currently, there is a growing number of chemical compounds that have been shown to block xenograft growth and inhibit HIF activity through a variety of molecular mechanisms such as decreased mRNA expression, protein synthesis and stabilization, subunit dimerization, DNA binding, and transcriptional activity of HIFs [11]. Antisense oligonucleotide (EZN2968) [70], and the aryl hydrocarbon receptor ligand aminoflavone have been shown to reduce HIF1 $\alpha$ mRNA levels [71]. Several agents reduce HIF1 $\alpha$ mRNA translation, including topoisomerase I inhibitors, such as EZN2208 [72], and mTOR inhibitors, temsirolimus and ridaforolimus [30,73]. HIF1 $\alpha$ protein stability can be targeted by 17DMAG, an inhibitor of the chaperone heat sock protein 90 (Hsp90) [74]. Although most published studies correspond to the regulation of HIF1 $\alpha$, many of these agents might also affect HIF $2 \alpha$ expression and activity [64].

Other HIF inhibitors have been discovered through phenotypic screens. A screen of drugs in clinical trials and/or use revealed that digoxin and other cardiac glycosides inhibit HIF1 $\alpha$ protein synthesis and expression of HIF1 $\alpha$ target genes in cancer cells [65]. In tumor xenografts, administration of digoxin increased latency and decreased growth, whereas treatment of established tumors resulted in growth arrest within one week [65]. Another screen using a cell-based reporter gene assay identified the anthracycline chemotherapeutic agents doxorubicin and daunorubicin as potent inhibitors of HIF $1 \alpha$-mediated gene transcription by blocking HIF binding to DNA [75].

Given the prominent role of mTOR in control of HIF expression, several mTOR inhibitors are under clinical investigation for patients with STS [68]. A large prospective study of ridaforolimus, an mTOR inhibitor, in 212 patients with metastatic or unresectable sarcomas showed a clinical benefit rate of $29 \%$ [73], which is greater than the expected rate with standard doxorubicin therapy [66]. Patients in the leiomyosarcoma and liposarcoma cohorts had slightly higher clinical benefit rates than those patients in the "other" cohort, highlighting the need for better biomarkers to identify patients who may respond [73]. A randomized, placebo-controlled Phase III study of ridaforolimus as maintenance therapy in 711 patients with advanced bone and soft tissue sarcomas who had at least stable disease following prior chemotherapy, showed an improvement in progression free survival as compared to the placebo group [73,76,77].

In addition, combining several HIF inhibitors may allow lower doses of individual agents, thereby reducing the likelihood of off-target effects, while still allowing for effective inhibition of the HIF pathway by multiple mechanisms [78]. In addition, combination treatment with HIF inhibitors may improve the efficacy of anti-VEGF therapy, by blocking compensatory pathways exploited by cancer cells to overcome environmental stresses [78]. To expand the use of agents targeting VEGF and HIFs in sarcoma patients, it is essential to understand better the complex role of HIFs in controlling sarcoma progression and metastasis and the biological effects of VEGF and HIF inhibition on chemo- and radiosensitivity in these tumors. 
Currently, there is insufficient data to identify specific STS subtypes or specific class of tumors within a subtype that are more likely to benefit from hypoxia-driven therapies. Although there are no validated predictive biomarkers for the response to hypoxia-target therapy, future research should lead to selection of patients more likely to respond [66]. Furthermore, molecular imaging techniques, such as positron emission tomography imaging with fluorinated nitroimidazoles, are being developed to better predict the response to hypoxia-target therapies [79].

\section{Conclusions}

Mounting evidence suggests that hypoxia-induced factors are important regulators in tumor progression, metastasis, and therapy resistance in soft tissue sarcomas. HIFs and HIF-mediated genes can be used as important prognosticators to stratify patients with sarcomas into groups with distinct clinical behavior improving selection of high-risk cancers for management. For their critical role in regulating sarcoma progression, HIFs and HIF-mediated genes may provide a brand new frontier to fight sarcoma. Better understanding of HIFs in regulating key oncogenic pathways will expand the use of agents targeting hypoxia-related genes in sarcoma patients. Pharmacological agents are being developed to target hypoxia-related pathways in human cancers, and this growing library of agents may hold therapeutic implications for treating both the primary sarcoma and metastatic disease. Future studies utilizing more accurate tumor models should tease out the effects of targeting the HIF pathway because different isoforms of HIF are likely to have complex and even opposing functions during different stages of tumor development. In addition, better molecular and imaging markers are needed to improve patient selection and treatment-response surveillance to parallel the use of HIF inhibitors.

\section{References}

1. Majmundar, A.J.; Wong, W.J.; Simon, M.C. Hypoxia-inducible factors and the response to hypoxic stress. Mol. Cell 2010, 40, 294-309.

2. Bertout, J.A.; Patel, S.A.; Simon, M.C. The impact of $\mathrm{O}_{2}$ availability on human cancer. Nat. Rev. Cancer 2008, 8, 967-975.

3. Semenza, G.L. Targeting HIF-1 for cancer therapy. Nat. Rev. Cancer 2003, 3, 721-732.

4. Nordsmark, M.; Alsner, J.; Keller, J.; Nielsen, O.S.; Jensen, O.M.; Horsman, M.R.; Overgaard, J. Hypoxia in human soft tissue sarcomas: Adverse impact on survival and no association with p53 mutations. Br. J. Cancer 2001, 84, 1070-1075.

5. Brizel, D.M.; Scully, S.P.; Harrelson, J.M.; Layfield, L.J.; Bean, J.M.; Prosnitz, L.R.; Dewhirst, M.W. Tumor oxygenation predicts for the likelihood of distant metastases in human soft tissue sarcoma. Cancer Res. 1996, 56, 941-943.

6. Keith, B.; Johnson, R.S.; Simon, M.C. HIF1alpha and HIF2alpha: Sibling rivalry in hypoxic tumour growth and progression. Nat. Rev. Cancer 2011, 12, 9-22.

7. Xia, X.; Lemieux, M.E.; Li, W.; Carroll, J.S.; Brown, M.; Liu, X.S.; Kung, A.L. Integrative analysis of HIF binding and transactivation reveals its role in maintaining histone methylation homeostasis. Proc. Natl. Acad. Sci. USA 2009, 106, 4260-4265. 
8. Heikkila, M.; Pasanen, A.; Kivirikko, K.I.; Myllyharju, J. Roles of the human hypoxia-inducible factor (HIF)-3alpha variants in the hypoxia response. Cell Mol. Life Sci. 2011, 68, 3885-3901.

9. Pouyssegur, J.; Dayan, F.; Mazure, N.M. Hypoxia signalling in cancer and approaches to enforce tumour regression. Nature 2006, 441, 437-443.

10. Siegel, R.; Naishadham, D.; Jemal, A. Cancer statistics, 2012. CA Cancer J. Clin. 2012, 62, $10-29$.

11. Semenza, G.L. Hypoxia-inducible factors: Mediators of cancer progression and targets for cancer therapy. Trends Pharmacol. Sci. 2012, 33, 207-214.

12. Wu, M.Z.; Tsai, Y.P.; Yang, M.H.; Huang, C.H.; Chang, S.Y.; Chang, C.C.; Teng, S.C.; $\mathrm{Wu}$, K.J. Interplay between HDAC3 and WDR5 is essential for hypoxia-induced epithelial-mesenchymal transition. Mol. Cell 2011, 43, 811-822.

13. Crosby, M.E.; Devlin, C.M.; Glazer, P.M.; Calin, G.A.; Ivan, M. Emerging roles of microRNAs in the molecular responses to hypoxia. Curr. Pharm. Des. 2009, 15, 3861-3866.

14. Detwiller, K.Y.; Fernando, N.T.; Segal, N.H.; Ryeom, S.W.; D’Amore, P.A.; Yoon, S.S. Analysis of hypoxia-related gene expression in sarcomas and effect of hypoxia on RNA interference of vascular endothelial cell growth factor A. Cancer Res. 2005, 65, 5881-5889.

15. Semenza, G.L. Defining the role of hypoxia-inducible factor 1 in cancer biology and therapeutics. Oncogene 2010, 29, 625-634.

16. Aebersold, D.M.; Burri, P.; Beer, K.T.; Laissue, J.; Djonov, V.; Greiner, R.H.; Semenza, G.L. Expression of hypoxia-inducible factor-1alpha: A novel predictive and prognostic parameter in the radiotherapy of oropharyngeal cancer. Cancer Res. 2001, 61, 2911-2916.

17. Birner, P.; Gatterbauer, B.; Oberhuber, G.; Schindl, M.; Rossler, K.; Prodinger, A.; Budka, H.; Hainfellner, J.A. Expression of hypoxia-inducible factor-1 alpha in oligodendrogliomas: Its impact on prognosis and on neoangiogenesis. Cancer 2001, 92, 165-171.

18. Enatsu, S.; Iwasaki, A.; Shirakusa, T.; Hamasaki, M.; Nabeshima, K.; Iwasaki, H.; Kuroki, M. Expression of hypoxia-inducible factor-1 alpha and its prognostic significance in small-sized adenocarcinomas of the lung. Eur. J. Cardiothorac. Surg. 2006, 29, 891-895.

19. Fillies, T.; Werkmeister, R.; van Diest, P.J.; Brandt, B.; Joos, U.; Buerger, H. HIF1-alpha overexpression indicates a good prognosis in early stage squamous cell carcinomas of the oral floor. BMC Cancer 2005, 5, 84.

20. Hoffmann, A.C.; Mori, R.; Vallbohmer, D.; Brabender, J.; Klein, E.; Drebber, U.; Baldus, S.E.; Cooc, J.; Azuma, M.; Metzger, R.; et al. High expression of HIF1a is a predictor of clinical outcome in patients with pancreatic ductal adenocarcinomas and correlated to PDGFA, VEGF, and bFGF. Neoplasia 2008, 10, 674-679.

21. Lidgren, A.; Hedberg, Y.; Grankvist, K.; Rasmuson, T.; Vasko, J.; Ljungberg, B. The expression of hypoxia-inducible factor 1alpha is a favorable independent prognostic factor in renal cell carcinoma. Clin. Cancer Res. 2005, 11, 1129-1135.

22. Chen, C.; Ma, Q.; Ma, X.; Liu, Z.; Liu, X. Association of elevated HIF-2alpha levels with low Beclin 1 expression and poor prognosis in patients with chondrosarcoma. Ann. Surg. Oncol. 2011, 18, 2364-2372. 
23. Chen, C.; Zhou, H.; Wei, F.; Jiang, L.; Liu, X.; Liu, Z.; Ma, Q. Increased levels of hypoxia-inducible factor-1alpha are associated with Bcl-xL expression, tumor apoptosis, and clinical outcome in chondrosarcoma. J. Orthop. Res. 2011, 29, 143-151.

24. Huang, J.H.; Lee, F.S.; Pasha, T.L.; Sammel, M.D.; Karakousis, G.; Xu, G.; Fraker, D.; Zhang, P.J. Analysis of HIF-1alpha and its regulator, PHD2, in retroperitoneal sarcomas: Clinico-Pathologic implications. Cancer Biol. Ther. 2010, 9, 303-311.

25. Shintani, K.; Matsumine, A.; Kusuzaki, K.; Matsubara, T.; Satonaka, H.; Wakabayashi, T.; Hoki, Y.; Uchida, A. Expression of hypoxia-inducible factor (HIF)-1alpha as a biomarker of outcome in soft-tissue sarcomas. Virchows Arch. 2006, 449, 673-681.

26. Hoffmann, A.C.; Danenberg, K.D.; Taubert, H.; Danenberg, P.V.; Wuerl, P. A three-gene signature for outcome in soft tissue sarcoma. Clin. Cancer Res. 2009, 15, 5191-5198.

27. Zhong, H.; de Marzo, A.M.; Laughner, E.; Lim, M.; Hilton, D.A.; Zagzag, D.; Buechler, P.; Isaacs, W.B.; Semenza, G.L.; Simons, J.W. Overexpression of hypoxia-inducible factor 1alpha in common human cancers and their metastases. Cancer Res. 1999, 59, 5830-5835.

28. Kilic, M.; Kasperczyk, H.; Fulda, S.; Debatin, K.M. Role of hypoxia inducible factor-1 alpha in modulation of apoptosis resistance. Oncogene 2007, 26, 2027-2038.

29. Das, B.; Tsuchida, R.; Malkin, D.; Koren, G.; Baruchel, S.; Yeger, H. Hypoxia enhances tumor stemness by increasing the invasive and tumorigenic side population fraction. Stem Cells 2008, 26, 1818-1830.

30. Wan, X.; Shen, N.; Mendoza, A.; Khanna, C.; Helman, L.J. CCI-779 inhibits rhabdomyosarcoma xenograft growth by an antiangiogenic mechanism linked to the targeting of mTOR/Hif-1alpha/VEGF signaling. Neoplasia 2006, 8, 394-401.

31. Rey, S.; Semenza, G.L. Hypoxia-Inducible factor-1-dependent mechanisms of vascularization and vascular remodelling. Cardiovasc. Res. 2010, 86, 236-242.

32. Jham, B.C.; Ma, T.; Hu, J.; Chaisuparat, R.; Friedman, E.R.; Pandolfi, P.P.; Schneider, A.; Sodhi, A.; Montaner, S. Amplification of the angiogenic signal through the activation of the TSC/mTOR/HIF axis by the KSHV vGPCR in Kaposi's sarcoma. PLoS One 2011, 6, e19103.

33. Cai, Q.; Murakami, M.; Si, H.; Robertson, E.S. A potential alpha-helix motif in the amino terminus of LANA encoded by Kaposi's sarcoma-associated herpesvirus is critical for nuclear accumulation of HIF-1 alpha in normoxia. J. Virol. 2007, 81, 10413-10423.

34. Cai, Q.L.; Knight, J.S.; Verma, S.C.; Zald, P.; Robertson, E.S. EC5S ubiquitin complex is recruited by KSHV latent antigen LANA for degradation of the VHL and p53 t umor suppressors. PLoS Pathog. 2006, 2, e116.

35. Corless, C.L.; Barnett, C.M.; Heinrich, M.C. Gastrointestinal stromal tumours: Origin and molecular oncology. Nat. Rev. Cancer 2011, 11, 865-878.

36. Antonescu, C.R.; Viale, A.; Sarran, L.; Tschernyavsky, S.J.; Gonen, M.; Segal, N.H.; Maki, R.G.; Socci, N.D.; DeMatteo, R.P.; Besmer, P. Gene expression in gastrointestinal stromal tumors is distinguished by KIT genotype and anatomic site. Clin. Cancer Res. 2004, 10, $3282-3290$.

37. Yun, Z.; Maecker, H.L.; Johnson, R.S.; Giaccia, A.J. Inhibition of PPAR gamma 2 gene expression by the HIF-1-regulated gene DEC1/Stra13: A mechanism for regulation of adipogenesis by hypoxia. Dev. Cell 2002, 2, 331-341. 
38. Lin, Q.; Lee, Y.J.; Yun, Z. Differentiation arrest by hypoxia. J. Biol. Chem. 2006, 281, 30678-30683.

39. Kim, Y.; Lin, Q.; Zelterman, D.; Yun, Z. Hypoxia-Regulated delta-like 1 homologue enhances cancer cell stemness and tumorigenicity. Cancer Res. 2009, 69, 9271-9280.

40. Aryee, D.N.; Niedan, S.; Kauer, M.; Schwentner, R.; Bennani-Baiti, I.M.; Ban, J.; Muehlbacher, K.; Kreppel, M.; Walker, R.L.; Meltzer, P.; et al. Hypoxia modulates EWS-FLI1 transcriptional signature and enhances the malignant properties of Ewing's sarcoma cells in vitro. Cancer Res. 2010, 70, 4015-4023.

41. Knowles, H.J.; Schaefer, K.L.; Dirksen, U.; Athanasou, N.A. Hypoxia and hypoglycaemia in Ewing's sarcoma and osteosarcoma: Regulation and phenotypic effects of Hypoxia-Inducible Factor. BMC Cancer 2010, 10, 372.

42. Mayer, A.; Hoeckel, M.; von Wallbrunn, A.; Horn, L.C.; Wree, A.; Vaupel, P. HIF-Mediated hypoxic response is missing in severely hypoxic uterine leiomyomas. Adv. Exp. Med. Biol. 2010, 662, 399-405.

43. Francis, P.; Namlos, H.M.; Muller, C.; Eden, P.; Fernebro, J.; Berner, J.M.; Bjerkehagen, B.; Akerman, M.; Bendahl, P.O.; Isinger, A.; et al. Diagnostic and prognostic gene expression signatures in $177 \mathrm{~s}$ oft tissue sarcomas: Hypoxia-induced transcription profile signifies metastatic potential. BMC Genomics 2007, 8, 73.

44. Mito, J.K.; Riedel, R.F.; Dodd, L.; Lahat, G.; Lazar, A.J.; Dodd, R.D.; Stangenberg, L.; Eward, W.C.; Hornicek, F.J.; Yoon, S.S.; et al. Cross species genomic analysis identifies a mouse model as undifferentiated pleomorphic sarcoma/malignant fibrous histiocytoma. PLoS One 2009, 4, e8075.

45. Skubitz, K.M.; Francis, P.; Skubitz, A.P.; Luo, X.; Nilbert, M. Gene expression identifies heterogeneity of metastatic propensity in high-grade soft tissue sarcomas. Cancer 2012, 118, 4235-4243.

46. Sullivan, R.; Graham, C.H. Hypoxia-Driven selection of the metastatic phenotype. Cancer Metastasis Rev. 2007, 26, 319-331.

47. Krishnamachary, B.; Berg-Dixon, S.; Kelly, B.; Agani, F.; Feldser, D.; Ferreira, G.; Iyer, N.; LaRusch, J.; Pak, B.; Taghavi, P.; et al. Regulation of colon carcinoma cell invasion by hypoxia-inducible factor 1. Cancer Res. 2003, 63, 1138-1143.

48. Erler, J.T.; Bennewith, K.L.; Nicolau, M.; Dornhofer, N.; Kong, C.; Le, Q.T.; Chi, J.T.; Jeffrey, S.S.; Giaccia, A.J. Lysyl oxidase is essential for hypoxia-induced metastasis. Nature 2006, 440, 1222-1226.

49. Lu, X.; Yan, C.H.; Yuan, M.; Wei, Y.; Hu, G.; Kang, Y. In vivo dynamics and distinct functions of hypoxia in primary tumor growth and organotropic metastasis of breast cancer. Cancer Res. 2010, 70, 3905-3914.

50. Muller, A.; Homey, B.; Soto, H.; Ge, N.; Catron, D.; Buchanan, M.E.; McClanahan, T.; Murphy, E.; Yuan, W.; Wagner, S.N.; et al. Involvement of chemokine receptors in breast cancer metastasis. Nature 2001, 410, 50-56.

51. Erler, J.T.; Bennewith, K.L.; Cox, T.R.; Lang, G.; Bird, D.; Koong, A.; Le, Q.T.; Giaccia, A.J. Hypoxia-Induced lysyl oxidase is a critical mediator of bone marrow cell recruitment to form the premetastatic niche. Cancer Cell 2009, 15, 35-44. 
52. Denny, W.A. Hypoxia-Activated prodrugs in cancer therapy: Progress to the clinic. Future Oncol. 2010, 6, 419-428.

53. Bertout, J.A.; Majmundar, A.J.; Gordan, J.D.; Lam, J.C.; Ditsworth, D.; Keith, B.; Brown, E.J.; Nathanson, K.L.; Simon, M.C. HIF2alpha inhibition promotes p53 pat hway activity, tumor cell death, and radiation responses. Proc. Natl. Acad. Sci. USA 2009, 106, 14391-14396.

54. Moeller, B.J.; Dreher, M.R.; Rabbani, Z.N.; Schroeder, T.; Cao, Y.; Li, C.Y.; Dewhirst, M.W. Pleiotropic effects of HIF-1 blockade on tumor radiosensitivity. Cancer Cell 2005, 8, 99-110.

55. Yoon, S.S.; Duda, D.G.; Karl, D.L.; Kim, T.M.; Kambadakone, A.R.; Chen, Y.L.; Rothrock, C.; Rosenberg, A.E.; Nielsen, G.P.; Kirsch, D.G.; et al. Phase II study of neoadjuvant bevacizumab and radiotherapy for resectable soft tissue sarcomas. Int. J. Radiat. Oncol. Biol. Phys. 2011, 81, 1081-1090.

56. Rohwer, N.; Dame, C.; Haugstetter, A.; Wiedenmann, B.; Detjen, K.; Schmitt, C.A.; Cramer, T. Hypoxia-Inducible factor 1alpha determines gastric cancer chemosensitivity via modulation of p53 and NF-kappaB. PLoS One 2010, 5, e12038.

57. Hao, J.; Song, X.; Song, B.; Liu, Y.; Wei, L.; Wang, X.; Yu, J. Effects of lentivirus-mediated HIF-1alpha knockdown on hypoxia-related cisplatin resistance and their dependence on p53 status in fibrosarcoma cells. Cancer Gene Ther. 2008, 15, 449-455.

58. Sullivan, R.; Pare, G.C.; Frederiksen, L.J.; Semenza, G.L.; Graham, C.H. Hypoxia-Induced resistance to anticancer drugs is associated with decreased senescence and requires hypoxia-inducible factor-1 activity. Mol. Cancer Ther. 2008, 7, 1961-1973.

59. Generali, D.; Buffa, F.M.; Berruti, A.; Brizzi, M.P.; Campo, L.; Bonardi, S.; Bersiga, A.; Allevi, G.; Milani, M.; Aguggini, S.; et al. Phosphorylated ERalpha, HIF-1alpha, and MAPK signaling as predictors of primary endocrine treatment response and resistance in patients with breast cancer. J. Clin. Oncol. 2009, 27, 227-234.

60. Nakamura, J.; Kitajima, Y.; Kai, K.; Hashiguchi, K.; Hiraki, M.; Noshiro, H.; Miyazaki, K. HIF-1alpha is an unfavorable determinant of relapse in gastric cancer patients who underwent curative surgery followed by adjuvant 5-FU chemotherapy. Int. J. Cancer 2010, 127, $1158-1171$.

61. Unruh, A.; Ressel, A.; Mohamed, H.G.; Johnson, R.S.; Nadrowitz, R.; Richter, E.; Katschinski, D.M.; Wenger, R.H. The hypoxia-inducible factor-1 alpha is a negative factor for tumor therapy. Oncogene 2003, 22, 3213-3220.

62. Zhao, F.; Mancuso, A.; Bui, T.V.; Tong, X.; Gruber, J.J.; Swider, C.R.; Sanchez, P.V.; Lum, J.J.; Sayed, N.; Melo, J.V.; et al. Imatinib resistance associated with BCR-ABL upregulation is dependent on HIF-1 alpha-induced metabolic reprograming. Oncogene 2010, 29, 2962-2972.

63. Wilson, W.R.; Hay, M.P. Targeting hypoxia in cancer therapy. Nat. Rev. Cancer 2011, 11, 393-410.

64. Rapisarda, A.; Melillo, G. Overcoming disappointing results with antiangiogenic therapy by targeting hypoxia. Nat. Rev. Clin. Oncol. 2012, 9, 378-390.

65. Zhang, H.; Qian, D.Z.; Tan, Y.S.; Lee, K.; Gao, P.; Ren, Y.R.; Rey, S.; Hammers, H.; Chang, D.; Pili, R.; et al. Digoxin and other cardiac glycosides inhibit HIF-1alpha synthesis and block tumor growth. Proc. Natl. Acad. Sci. USA 2008, 105, 19579-19586. 
66. Ganjoo, K.N.; Cranmer, L.D.; Butrynski, J.E.; Rushing, D.; Adkins, D.; Okuno, S.H.; Lorente, G.; Kroll, S.; Langmuir, V.K.; Chawla, S.P. A p hase I study of the safety and pharmacokinetics of the hypoxia-activated prodrug TH-302 in combination with doxorubicin in patients with advanced soft tissue sarcoma. Oncology 2011, 80, 50-56.

67. Ganjoo, K.N. New developments in targeted therapy for soft tissue sarcoma. Curr. Oncol. Rep. 2010, 12, 261-265.

68. Moyer, M.W. Targeting hypoxia brings breath of fresh air to cancer therapy. Nat. Med. 2012, 18, 636-637.

69. Ma, W.W.; Adjei, A.A. Novel agents on the horizon for cancer therapy. CA Cancer J. Clin. 2009, 59, 111-137.

70. Greenberger, L.M.; Horak, I.D.; Filpula, D.; Sapra, P.; Westergaard, M.; Frydenlund, H.F.; Albaek, C.; Schroder, H.; Orum, H. A RNA antagonist of hypoxia-inducible factor-1alpha, EZN-2968, inhibits tumor cell growth. Mol. Cancer Ther. 2008, 7, 3598-3608.

71. Terzuoli, E.; Puppo, M.; Rapisarda, A.; Uranchimeg, B.; Cao, L.; Burger, A.M.; Ziche, M.; Melillo, G. Aminoflavone, a 1 igand of the aryl hydrocarbon receptor, inhibits HIF-1alpha expression in an AhR-independent fashion. Cancer Res. 2010, 70, 6837-6848.

72. Sapra, P.; Kraft, P.; Pastorino, F.; Ribatti, D.; Dumble, M.; Mehlig, M.; Wang, M.; Ponzoni, M.; Greenberger, L.M.; Horak, I.D. Potent and s ustained inhibition of HIF-1alpha and downstream genes by a po lyethyleneglycol-SN38 conjugate, EZN-2208, results in anti-angiogenic effects. Angiogenesis 2011, 14, 245-253.

73. Chawla, S.P.; Staddon, A.P.; Baker, L.H.; Schuetze, S.M.; Tolcher, A.W.; D’Amato, G.Z.; Blay, J.Y.; Mita, M.M.; Sankhala, K.K.; Berk, L.; et al. Phase II study of the mammalian target of rapamycin inhibitor ridaforolimus in patients with advanced bone and soft tissue sarcomas. J. Clin. Oncol. 2012, 30, 78-84.

74. Isaacs, J.S.; Jung, Y.J.; Mimnaugh, E.G.; Martinez, A.; Cuttitta, F.; Neckers, L.M. Hsp90 regulates a $\mathrm{v}$ on Hippel Lindau-independent hypoxia-inducible factor-1 alpha-degradative pathway. J. Biol. Chem. 2002, 277, 29936-29944.

75. Lee, K.; Qian, D.Z.; Rey, S.; Wei, H.; Liu, J.O.; Semenza, G.L. Anthracycline chemotherapy inhibits HIF-1 transcriptional activity and tumor-induced mobilization of circulating angiogenic cells. Proc. Natl. Acad. Sci. USA 2009, 106, 2353-2358.

76. Keedy, V.L. Treating metastatic soft-tissue or bone sarcomas-Potential role of ridaforolimus. Oncol. Targets Ther. 2012, 5, 153-160.

77. Mita, M.M.; Poplin, E.; Britten, C.D.; Tap, W.D.; Rubin, E.H.; Scott, B.B.; Berk, L.; Rivera, V.M.; Loewy, J.W.; Dodion, P.; et al. Phase I/IIa trial of the mammalian target of rapamycin inhibitor ridaforolimus (AP23573; MK-8669) administered orally in patients with refractory or advanced malignancies and sarcoma. Ann. Oncol. 2013, 24, 1104-1111.

78. Rapisarda, A.; Shoemaker, R.H.; Melillo, G. Antiangiogenic agents and HIF-1 inhibitors meet at the crossroads. Cell Cycle 2009, 8, 4040-4043.

79. Krohn, K.A.; Link, J.M.; Mason, R.P. Molecular imaging of hypoxia. J. Nucl. Med. 2008, 49, 129S-148S. 
Reprinted from Cancers. Cite as Bernthal, N.M.; Jones, K.B.; Monument, M.J.; Liu, T.; Viskochil, D.; Randall, R.L. Lost in Translation: Ambiguity in Nerve Sheath Tumor Nomenclature and Its Resultant Treatment Effect. Cancers 2013, 5, 519-528.

Review

\title{
Lost in Translation: Ambiguity in Nerve Sheath Tumor Nomenclature and Its Resultant Treatment Effect
}

\author{
Nicholas M. Bernthal ${ }^{1}$ *, Kevin B. Jones ${ }^{1}$, Michael J. Monument ${ }^{1}$, Ting Liu ${ }^{2}$, \\ David Viskochil ${ }^{3}$ and R. Lor Randall ${ }^{1} *$
}

1 Sarcoma Services, Department of Orthopaedics, Huntsman Cancer Institute and Primary Childrens Medical Center, University of Utah, Salt Lake City, UT 84112, USA

2 Department of Pathology, Huntsman Cancer Institute, University of Utah, Salt Lake City, UT 84112, USA

3 Division of Medical Genetics, Department of Pediatrics, University of Utah, Salt Lake City, UT 84112, USA

* Authors to whom correspondence should be addressed;

E-Mails: Nicholas.bernthal@hsc.utah.edu (N.B.); Lor.Randall@hci.utah.edu (R.L.R.); Tel.: +1-301-518-7147 (N.B.).

Received: 4 March 2013; in revised form: 9 April 2013 / Accepted: 3 May 2013 /

Published: 8 May 2013

\begin{abstract}
There is much ambiguity surrounding the diagnosis of nerve sheath tumors, including atypical neurofibroma and low-grade MPNST, and yet, the distinction between these entities designates either benign or malignant behavior and thus carries presumed profound prognostic importance that often guides treatment. This study reviews the diagnostic criteria used to designate atypical neurofibroma from low-grade MPNSTs and reviews existing literature the natural history of each of these tumors to see if the distinction is, in fact, of importance.
\end{abstract}

Keywords: MPNST; neurofibromatosis; nerve sheath tumor; atypical neurofibroma; low grade MPNST

\section{Introduction}

Peripheral nerve sheath tumors (PNSTs) comprise a spectrum of neoplastic potential ranging from benign neurofibromas and schwannomas to high-grade malignant peripheral nerve sheath tumors 
(MPNSTs). Benign neurofibromas are often found incidentally and usually require only symptomatic treatment. In contrast, high-grade MPNSTs are fulminantly malignant lesions with clinical outcomes on par with the worst of soft tissue sarcomas, despite aggressive treatment. Along this spectrum, atypical neurofibromas and low-grade MPNSTs reside in an undefined, nebulous middle ground that presents a treatment challenge to physicians and surgeons. While convention defines atypical neurofibromas as benign lesions and low-grade MPNSTs as malignant, this distinction may not be so clear. In this analysis, we review the present literature on atypical neurofibromas and low-grade MPNSTs to evaluate the clinical utility of this distinction. In doing so, we hope to help guide treatment for these challenging tumors.

\section{Methods}

A PUBMED search was performed on 20 September 2012, searching for the following key words: "atypical neurofibroma", "malignant peripheral nerve sheath tumor", "low-grade MPNST", and "neurofibromatosis". Articles from 1970-2012 were reviewed.

\section{A Rose by Any Other Name [1]}

\subsection{Neurofibroma}

According to the World Health Organization (WHO), a neurofibroma is an unencapsulated nerve sheath tumor comprised of a heterogenous population of cells of mixed origin in which Schwann cells are the predominant cell type [2]. Neurofibromas are the most common type of peripheral nerve sheath tumor and approximately $10 \%$ are associated with the genetic disorder neurofibromatosis type 1 (NF1) [3]. Neurofibromas are often described as "benign, slow-growing, and often painless" and treatment is predominantly surgical resection of those tumors that are symptomatic [4]. Neurofibromas are often subclassified into localized, diffuse and plexiform types based on their gross appearance and location [2]. Morphologically, neurofibromas are generally described as having mild nuclear size and hyperchromasia, minimal mitotic activity and no areas of necrosis [5]. The spindly cells show wavy, small and dark nuclei without cytological atypia. Intermixed are dense, wire-like collagen, often described as "shredded carrot" type. A myxoid or mucin rich stroma matrix is noted (Figure 1a,b).

\subsection{Malignant Peripheral Nerve Sheath Tumors (MPNSTs)}

MPNSTs are defined by the WHO as "any malignant tumor arising from a peripheral nerve or showing nerve sheath differentiation, excluding those originating from epineurium or peripheral nerve vasculature" [2]. This broad definition encompasses tumors previously classified as neurogenic sarcoma, malignant schwannoma, malignant neurilemoma, and neurofibrosarcoma [4]. While several variants have been described including epithelioid, glandular, and rhabdomyosarcomatous (triton tumor), high-grade MPNSTs are generally thought to grow rapidly, cause severe pain, and lead to "relentlessly progressive neurologic deficits" [4]. In contrast to neurofibromas, MPNSTs are histologically characterized by a fascicular arrangement of grossly atypical wavy spindle cells with an increased nuclear:cytoplasm ratio, a high mitotic index, minimal 
"shredded carrot" type collagen, high mitotic activity, a fascicular growth pattern, and prominent areas of necrosis [5] (Figure 2a,b).

Figure 1. An intermediate $(200 \times)$ (a) and high power view $(400 \times)$; (b) of routine hematoxylin and eosin (H\&E stain) sections of typical neurofibromas from an NF1 patient. A low cellularity of spindly (Schwann) cells intermixed with abundant stroma mucin and prominent "shredded carrot" collagen. There is no evidence of cytologic atypia, increased cellularity, or mitotic figures.

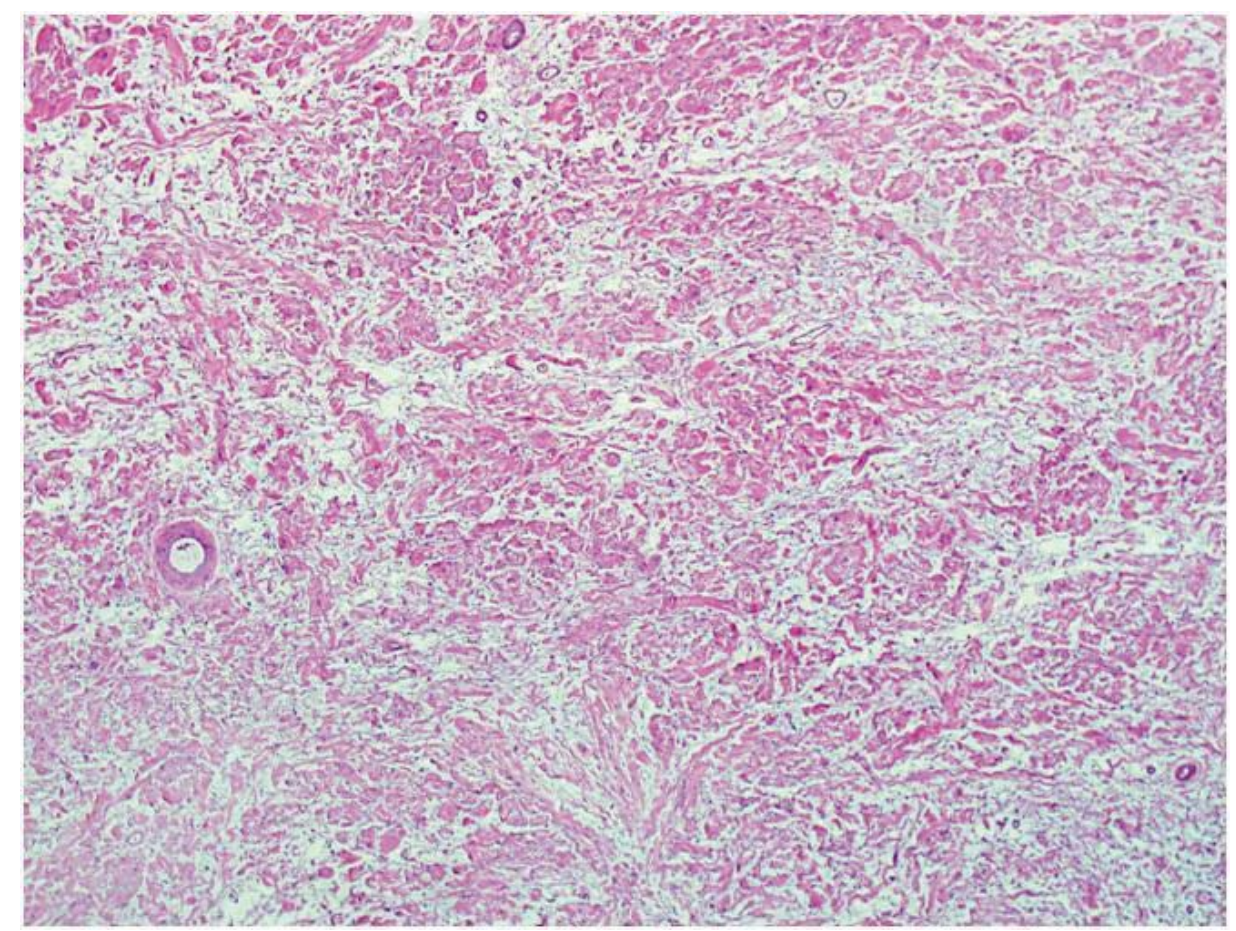

(a)

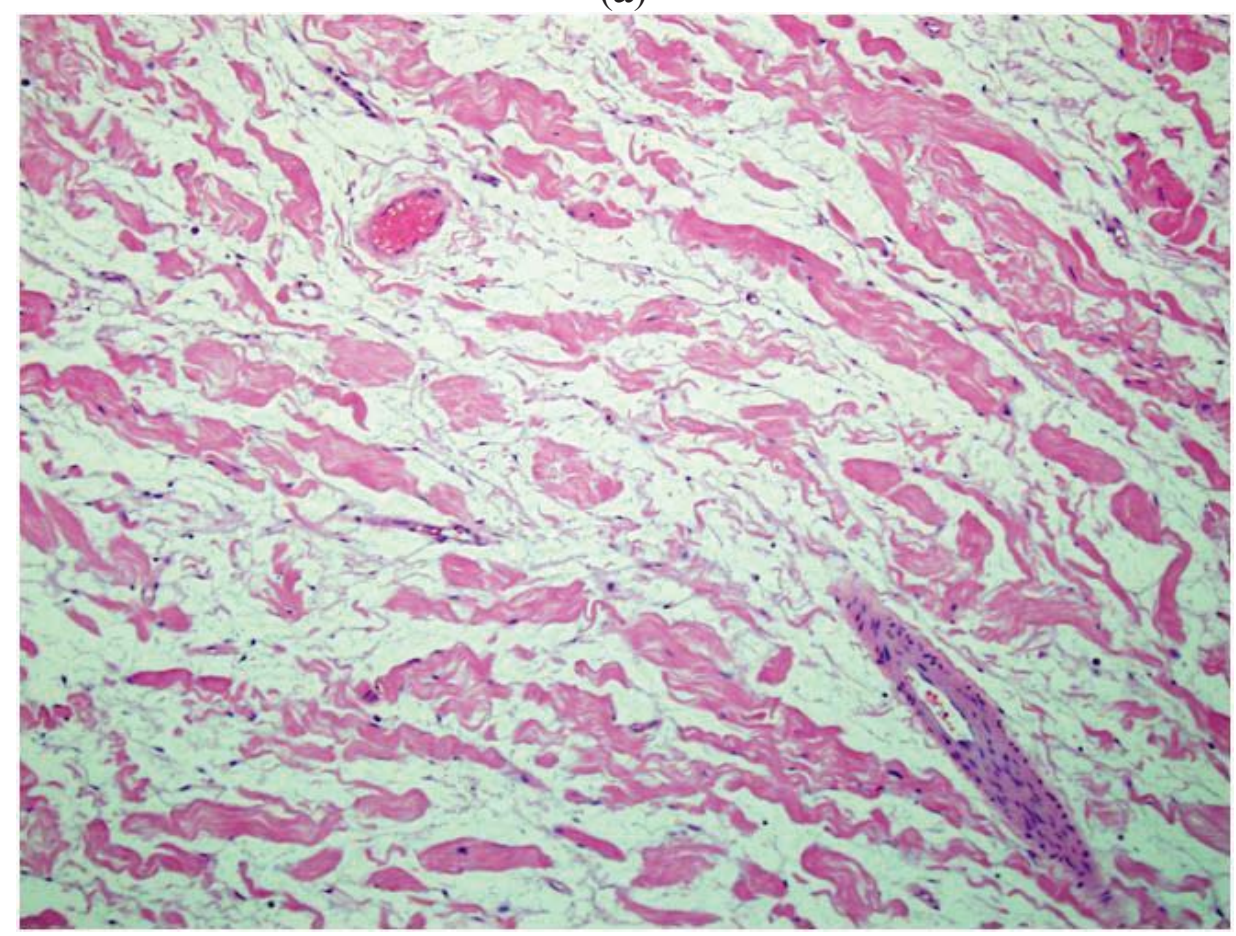

(b) 
Figure 2. An intermediate power $(200 \times)$ (a) view of routine hematoxylin and eosin (H\&E stain) sections of a high-grade MPNST from an NF1 patient. A high cellularity of spindly (Schwann) cells intermixed with a fascicular growth pattern and little "shredded carrot" collagen. High power (400×); (b) view shows increased cellularity, atypia, and mitotic figures.

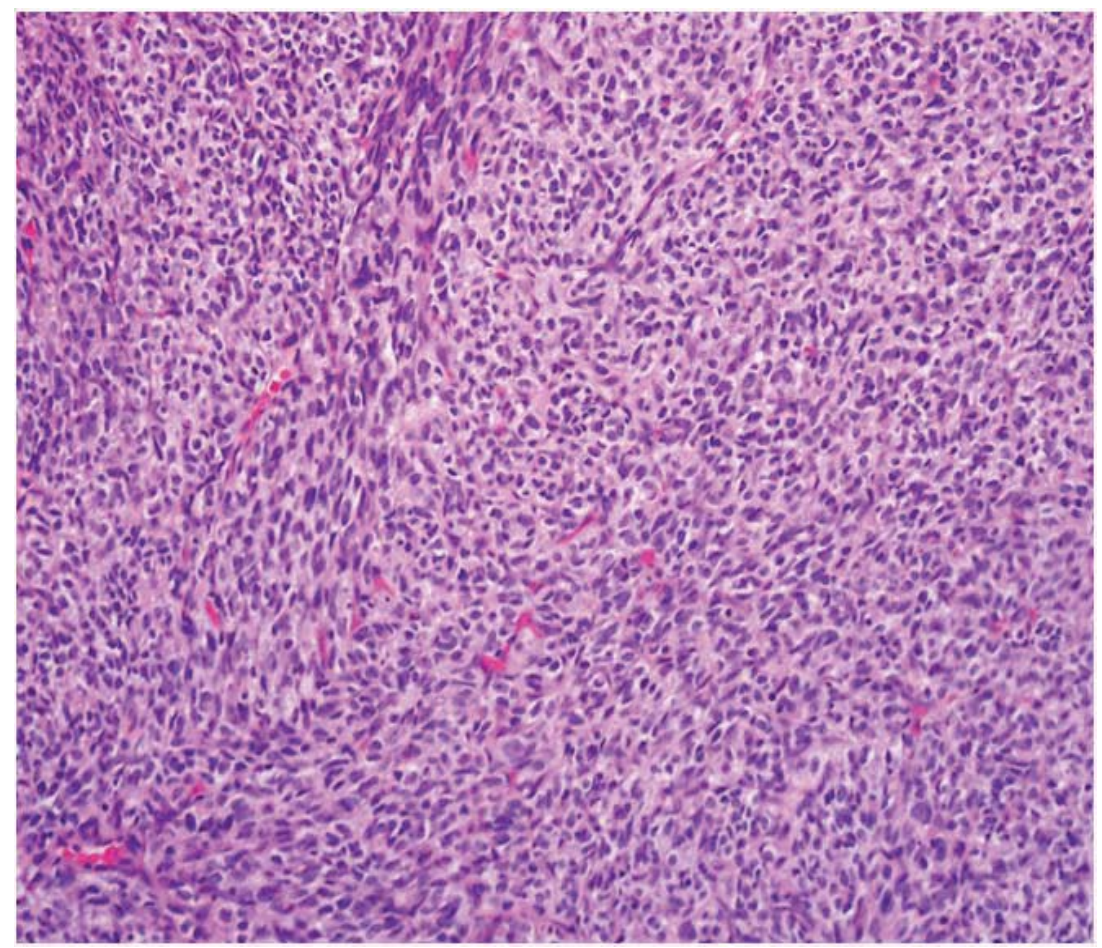

(a)

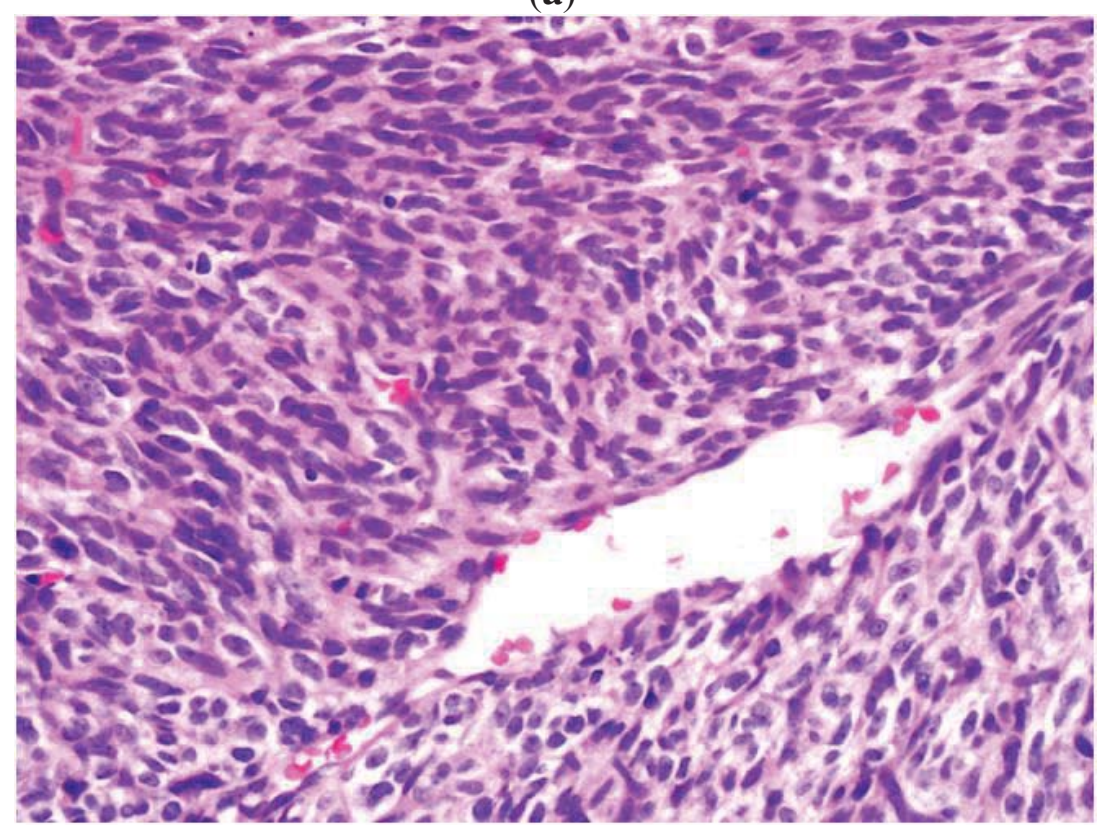

(b)

\section{Shades of Grey}

Unfortunately for physicians and patients alike, the aforementioned pathohistological criteria used to differentiate neurofibroma from MPNST are most effective at differentiating "typical" 
neurofibromas from high-grade MPNSTs. However, the intermediate lesions, the atypical neurofibromas and low-grade MPNSTs, have characteristics of both tumors, and therefore present a difficult diagnostic and treatment conundrum [6].

\subsection{Atypical Neurofibroma}

An atypical neurofibroma is classified as such based on increased cellularity, more degenerative cytological atypia, and a more pronounced fascicular growth pattern as compared to the more "typical" neurofibroma. While all of these characteristics are more suggestive of MPNST, atypical neurofibromas lack the "monotonous" cytological atypia, chromatin abnormalities and mitotic activity seen in MPNST [5].

\subsection{Low-Grade MPNST}

An MPNST is histologically defined as a hy percellular tumor with nuclear enlargement, hyperchromasia, and a pronounced fascicular growth pattern (similar to atypical neurofibroma). A low-grade MPNST is one in which less than five mitoses per 10 high-power fields are noted in the setting of the aforementioned signs of malignancy [5]. Further, necrosis is conspicuously lacking.

\section{Imaging}

Positron-emission/computerized tomography (PET/CT) has been applied to help distinguish benign and malignant nerve sheath tumors [7]. The mean standardized uptake value (SUV) in benign neurofibroma has been calculated in one series at 1.5 (SD 1.1) as compared to 5.7 (SD 2.6) in MPNST [8]. However, again the intermediate lesions muddy the waters: recent work applying the aforementioned scale to determine malignancy shows an atypical neurofibroma in a patient with NF1 with a high, "malignant-level" SUV of 5.7 on PET/CT [9].

\section{Choose Wisely...}

The rather ambiguous classification noted above, distinguishing atypical neurofibroma from low-grade MPNST is an ostensibly critical one in guiding treatment. A low-grade MPNST is, by definition, a malignant lesion, and therefore convention suggests treatment with wide resection in an attempt for oncologic cure. Conversely, an atypical neurofibroma, a benign lesion, can be treated with intralesional or marginal resection if a wide margin carries significant morbidity. With this major threshold-initiated shift in treatment paradigms in mind, getting the classification "correct" becomes of utmost significance.

The situation becomes more complex when one examines the clinical behavior of nerve sheath tumors. While treatment is often guided reflexively by the categorizing of a tumor as benign or malignant, the natural history of low-grade MPNSTs and of atypical neurofibromas must be considered. In other words, do low grade MPNSTs truly exhibit malignant behavior and are atypical neurofibromas actually benign? This question drives the present review. 


\section{Clinical Outcomes}

Studies looking at MPNSTs often aggregate low- and high-grade lesions into one category of "Malignant Peripheral Nerve Sheath Tumor." This grouping clearly skews clinical results to assign a more "malignant" course for low-grade MPNSTs than may be justified. Similarly, studies looking at neurofibromas often include both typical and atypical neurofibromas, thereby raising the possibility of "diluting" a more aggressive natural history of atypical neurofibromas. We therefore reviewed existing literature on low-grade MPNSTs and atypical neurofibromas to see if these lesions are clinically distinguishable at two separate intermediately aggressive groups.

The largest study to date of MPNSTs is a single-institution review of 205 patients with localized MPNST treated with surgery at the Nazionale per lo Studio e la Cura dei Tumori in Milan, Italy [10]. While the report's discussion concludes that tumor grade is not a significant factor for survival, close analysis of the data brings this conclusion into doubt. For the statistical analysis, the authors combine low and intermediate grade MPNSTs as a single group, then compare these to high-grade tumors, concluding that the groups do not differ in survival. Intermediate grade MPNST in the French Grading system used in this cohort includes tumors with a total score of $4-5$, which could include a tumor that is completely undifferentiated (3 points) and has 10-19 mitoses per 10 HPF (2 points) [11]. Such a so-called intermediate grade tumor has more in common histologically with a high-grade MPNST than a low-grade MPNST. Such blurred distinctions may well distort a survival comparison. When low-grade tumors are reviewed as an independent group, only $6 \%$ of the cohort, or 14 total patients, can be considered. One of these 14 patients (7\%) developed local recurrence and metastatic disease, ultimately dying of disease. The authors did not designate whether this patient had NF1, which might indicate development of a second MPNST, rather than a true recurrence of the first tumor.

A recent publication from the Mayo Clinic reviewed the institution's experience with 175 MPNST patients [12]. This study classifies MPNSTs on a four-tier grading system, with grade 1 and 2 tumors (well differentiated and intermediately differentiated) as "low grade" and grade 3 and 4 tumors as "high grade." They conclude that tumor grade was significantly correlated with disease specific survival on uni- and multi-variate analysis. The 50 patients with grade 1 or 2 "low grade" MPNSTs included seven who developed metastatic disease (14\%). This again includes "intermediately differentiated" tumors as low-grade but similar to the Italian cohort, cannot rule out the possibility of aggressive behavior in "low-grade" lesions.

Unfortunately, the remaining literature addressing outcomes of low-grade MPNSTs is limited to small case series. Okada et al. published a series of 55 cases of MPNST, in which six were classified as low-grade by AJCC classification [13]. All six host patients showed no evidence of disease at final follow-up after resection. As a diagnostic aside, the authors note that all low-grade MPNSTs were S100 positive in their cohort whereas only 50\% of high-grade MPNSTs demonstrated S100 positivity. Yamaguchi et al. published a retrospective case series of four cases of low-grade MPNSTs (AJCC classification) [14]. In this study, there were no recurrences, metastases or deaths attributable to disease in these patients with low-grade tumors.

These four studies represent the complete literature available for low-grade MPNSTs, paltry as it is. Atypical neurofibromas have even less conclusive data available than low-grade MPNSTs. The term, "atypical neurofibroma" is most often associated with a neurofibroma in the setting of a patient 
with NF1 and has recently been described by several studies as a "premalignant lesion in transit", i.e., in the early stages of transitioning from a benign neurofibroma to an aggressive MPNST. While some cytogenetic analyses were recently published providing support for this hypothesis [15], there are no published articles currently available in PUBMED showing clinical/oncologic outcomes of atypical neurofibromas.

\section{Discussion}

The spectrum of neoplastic potential ranging from benign neurofibromas to MPNSTs continues to challenge our diagnostic and therapeutic capabilities today. Between the biological extremes - a benign neurofibroma producing only local symptoms and a high-grade MPNST progressing rapidly to metastasis and death despite aggressive treatment — most lesions lie somewhere in the middle range. Evidence for a correlation between histologic features and ultimate clinical course is rather weak at any level, but downright absent for the graded distinctions between atypical neurofibromas and low-grade MPNSTs.

This ambiguity challenges the patient and the treating physician. Tailored treatment options along a range of aggressiveness are not really available. We basically have non-operative surveillance, marginal, nerve-sparing excisions, and wide, morbid resections in our arsenal. Because ultimately high-grade tumors are so profoundly recalcitrant to any treatment options, any tumor known to be on a biological course headed that direction would merit aggressive and morbid treatment while it is yet possible. Conversely, any tumor known to be stable in a non-progressive state would be happily observed over time, or symptomatically managed with a minimally-morbid resection. The distinction is of paramount importance, but remains woefully unclear. The question is highlighted in NF1 patients, in whom "atypical" neurofibromas often track along the nerve sheath of an entire extremity all the way to the nerve roots at the spinal cord. Massively disfiguring and debilitating surgeries are often performed on these patients in the name of oncologic principles with no clear information available regarding their biology or natural history.

Future work aims to address this ambiguity in two ways. First, the science of molecular genetics is rapidly advancing and may provide us a better understanding of what truly defines a "malignant" nerve sheath tumor. Recent cytogenetic data suggests that mutations in the CDKN2A and CDKN2B loci, which code for cell cycle regulators p16 and p14, accumulate as part of the "process" by which neurofibromas become malignant [15]. Other work suggests that MPNSTs arise from precursors via silencing mutations in multiple tumor suppressor genes (e.g., TP53, CDKN2A) and amplifications of tyrosine kinase receptor genes (e.g., EGFR) [16-19]. A recent case report presents genomic changes in one area of a neurofibroma that "de-differentiated" to a MPNST, where losses of TP53, RB1, and CDKN2A were found only in the malignant portion of the tumor [20]. Additional work has been done attempting to correlate MDM2 amplification with p53 immunoreactivity to better distinguish among nerve sheath tumors, but with little proposed current applicability [21]. Nonetheless, future molecular diagnostic analyses will undoubtedly assist us to define the lesions more accurately than we are currently able to with histopathology and immunohistochemistry.

The second area for future work lies in well-organized clinical outcome studies. Our institution, among others, is in the process of analyzing the clinical outcomes of atypical neurofibromas and low-grade MPNSTs. With widely agreed upon criteria and a common grading system, large-scale, 
multi-institutional studies could more clearly define the discernible risk factors (histopathologic or molecular) for a bad prognosis meriting more aggressive treatment.

\section{Conclusions}

While the distinction among nerve sheath tumors remains diagnostically challenging with current criteria, the importance of the distinction in determining the malignant potential of these tumors remains unclear in the current literature. Previous clinical studies have used inconsistent definitions and diagnostic criteria; thus, coalescing current data into a meta-analysis is not feasible. Molecular genetics will hopefully provide more reproducible definitions of these intermediate lesions and therefore allow more accurate assessment of natural history. This is essential prior to determining the appropriateness of aggressive and often debilitating surgical procedures.

\section{References}

1. Shakespeare, W. Romeo and Juliet. William Shakespeare Info. Available online: http://www.william-shakespeare.info/quotes-quotations-play-romeo-andjuliet.htm/ (accessed on 12 December 2012).

2. Kleihues, P.; Cavenee, W.K. World Health Organization Classification of Tumors: Pathology and Genetics of Tumorous of the Nervous System; AZRC Press: Lyon, France, 2000.

3. Kransdorf, M.; Murphay, M.D. Neurogenic tumors. In Imaging of Soft Tissue Tumors; Saunders Press: Philadelphia, PA, USA, 1997; pp. 235-273.

4. Huang, J.; Johnson, V.; Zager, E. Tumors of the peripheral nerves and plexuses. Curr. Treat. Opt. Neuro. 2006, 8, 299-308.

5. Rodriguez, F.; Folpe, A.; Giannini, C.; Perry, A. Pathology of peripheral nerve sheath tumors: Diagnostic overview and update on selected diagnostic problems. Acta Neuropathol. 2012, 123, 295-319.

6. Zhou, H.; Coffin, C.; Perkins, S.; Tripp, S.; Liew, M.; Viskochil, D. Malignant peripheral nerve sheath tumor: A comparison of grade, immunophenotype, and cell cycle/growth activation marker expression in sporadic and neurofibromatosis 1-related lesions. Am. J. Surg. Pathol. 2003, 27, 1337-1345.

7. Ferner, R.; Lucas, J.; O’Doherty, M.; Hughes, R.; Smith, M.; Cronin, B.; Bingham, J. Evaluation of ${ }^{18}$ fluorodeoxyglucose positron-emission tomography $\left({ }^{18}\right.$ FDG-PET) in the detection of malignant peripheral nerve sheath tumors arising from within plexiform neurofibromas in neurofibromatosis 1. J. Neurol. Neurosurg. Psychiatry 2000, 68, 353-357.

8. Ferner, R.; Golding, J.; Smith, M.; Calonje, E.; Jan, W.; Sanhayanathan, V.; O’Doherty, M. $\left({ }^{18} \mathrm{~F}\right) 2$-Fluoro-deoxy-D-glucose positron emission tomography (FDG-PET) as a diagnostic tool for neurofibromatosis 1 (NF1) associated malignant peripheral nerve sheath tumors (MPNSTs): A long-term clinical study. Ann. Oncol. 2008, 19, 390-394.

9. Friedrich, R.; Derlin, T.; Hagel, C. Atypical plexiform neurofibroma in NF1 with high Standardized Uptake Value (SUV) in Positron-Emission Tomography (PET) expressing podoplanin. In Vivo 2010, 6, 871-876. 
10. Anghileri, M.; Miceli, R.; Fiore, M.; Mariani, L.; Ferrari, A.; Mussi, C.; Lozza, L.; Collini, P.; Olmi, P.; Casali, P.; et al. Malignant peripheral nerve sheath tumors: Prognostic factors and survival in a series of patients treated at a single institution. Cancer 2006, 107, 1065-1074.

11. Trojani, M.; Contesso, G.; Coindre, J.; Rouesse, J.; Bui, N.; de Mascarel, A.; Goussot, J.; David, M.; Bonichon, F.; Lagarde, C. Soft-tissue sarcomas of adults; study of pathological prognostic variables and definition of a histopathological grading system. Int. J. Cancer 1984, $33,37-42$.

12. Stucky, C.; Johnson, K.; Gray, R.; Pockaj, B.; Ocal, I.; Rose, P.; Wasif, N. Malignant Peripheral Nerve Sheath Tumors (MPNST): The mayo clinic experience. Ann. Surg. Oncol. 2012, 19, 878-885.

13. Okada, K.; Hasegawa, T.; Tajino, T.; Hotta, T.; Yanagisawa, M.; Osanai, T.; Nishida, J.; Seki, K.; Itoi, E. Clinical relevance of pathological grades of malignant peripheral nerve sheath tumor: A multi-institutional TMTS study of $56 \mathrm{c}$ ases in Northern Japan. Ann. Surg. Oncol. 2007, 14, 597-604.

14. Yamaguchi, U.; Hasegawa, T.; Hirose, T.; Chuman, H.; Kawai, A.; Ito, Y.; Beppu, Y. Low grade malignant peripheral nerve sheath tumor: Varied cytological and histological patterns. J. Clin. Pathol. 2003, 56, 826-830.

15. Beert, E.; Brems, H.; Daniels, B.; de Wever, I.; van Calenbergh, F.; Schoenaers, J.; Debiec-Rychter, M.; Gevaert, O.; de Raedt, T.; van den Bruel, A.; et al. Atypical neurofibromas in neurofibromatosis type I are premalignant tumors. Genes Chromosom. Cancer 2011, 50, 1021-1032.

16. Menon, A.; Anderson, K.; Riccardi, V.; Chung, R.; Whaley, J.; Yandell, D.; Farmer, G.; Friedman, R.; Lee, J.; Li, F.; et al. Chromosome 17p de letions and p53 gen e mutations associated with the formation of malignant neurofibrosarcomas in von recklinghausen neurofibromastosis. Proc. Natl. Acad. Sci. USA 1990, 14, 5435-5439.

17. Kourea, H.; Orlow, I.; Scheithauer, B.; Cordon-Cardo, C.; Woodruff, J. Deletions of the INK4A gene occur in malignant peripheral nerve sheath tumors but not in neurofibromas. Am. J. Pathol. 1999, 155, 1855-1860.

18. Nielsen, G.; Stemmer-Rachaminov, A.; Ino, Y.; Moller, M.; Rosenberg, A.; Louis, D. Malignant transformation of neurofibromas in neurofibromatosis 1 is associated with CDKN2A/p16 inactivation. Am. J. Pathol. 1999, 155, 1879-1884.

19. Perry, A.; Kunz, S.; Fuller, C.; Banerjee, R.; Marley, E.; Liapis, H.; Watson, M.; Gutmann, D. Differential NF1, p16, and EGFR patterns by interphase cytogenetics (FISH) in Malignant Peripheral Nerve Sheath Tumor (MPNST) and morphologically similar spindle cell neoplasms. J. Neuropathol. Exp. Neurol. 2002, 61, 702-709.

20. Spurlock, G.; Knight, S.J.L.; Thomas, N.; Kiehl, T.; Guha, A.; Upadhyaya, M. Molecular evolution of a neurofibroma to Malignant Nerve Sheath Tumor (MPNST) in an NF1 Patient: Correlation between histopathological, clinical, and molecular findings. J. Cancer Res. Clin. Oncol. 2010, 136, 1869-1880.

21. Wallander, M.; Tripp, S.; Layfield, L. MDM2 amplification in malignant peripheral nerve sheath tumors correlates with p53 protein expression. Arch. Pathol. Lab. Med. 2012, 136, 95-99. 
Reprinted from Cancers. Cite as Boerkamp, K.M.; Rutteman, G.R.; Kik, M.J.L.; Kirpensteijn, J.; Schulze, C.; Grinwis, G.C.M. Nuclear DNA-Content in Mesenchymal Lesions in Dogs: Its Value as Marker of Malignancy and Extent of Genomic Instability. Cancers 2012, 4, 1300-1317.

Article

\title{
Nuclear DNA-Content in Mesenchymal Lesions in Dogs: Its Value as Marker of Malignancy and Extent of Genomic Instability
}

\author{
Kim M. Boerkamp ${ }^{1, \uparrow}$, , Gerard R. Rutteman ${ }^{1, \dagger}$, Marja J. L. Kik ${ }^{2}$, Jolle Kirpensteijn ${ }^{1}$, \\ Christoph Schulze $^{2, *}$ and Guy C. M. Grinwis ${ }^{2}$
}

1 Department of Clinical Science of Companion Animals, Faculty of Veterinary Medicine, UU, Yalelaan 104, 3584 CM, Utrecht, The Netherlands; E-Mails: G.R.Rutteman@uu.nl (G.R.R.); J.Kirpensteijn@uu.nl (J.K.)

2 Department of Pathobiology, Faculty of Veterinary Medicine, UU, Yalelaan 1, 3508 TD, Utrecht, The Netherlands; E-Mails: M.Kik@uu.nl (M.J.L.K.); Christoph.Schulze@Landeslabor-bbb.de (C.S.); Grinwis@uu.nl (G.C.M.G.)

$\dagger$ These authors contributed equally to this work.

Appointment at time of investigation.

* Author to whom correspondence should be addressed; E-Mail: K.M.Boerkamp@uu.nl; Tel.: +31-30-253-5243; Fax: +31-30-251-8126.

Received: 25 October 2012; in revised form: 16 November 2012 / Accepted: 26 November 2012 / Published: 3 December 2012

\begin{abstract}
DNA-aneuploidy may reflect the malignant nature of mesenchymal proliferations and herald gross genomic instability as a mechanistic factor in t umor genesis. DNA-ploidy and -index were determined by flow cytometry in canine inflammatory or neoplastic mesenchymal tissues and related to clinico-pathological features, biological behavior and p53 gene mutational status. Half of all sarcomas were aneuploid. Benign mesenchymal neoplasms were rarely aneuploid and inflammatory lesions not at all. The aneuploidy rate was comparable to that reported for human sarcomas with significant variation amongst subtypes. DNA-ploidy status in canines lacked a relation with histological grade of malignancy, in contrast to human sarcomas. While aneuploidy was related to the development of metastases in soft tissue sarcomas it was not in osteosarcomas. No relation amongst sarcomas was found between ploidy status and presence of P53 gene mutations. Heterogeneity of the DNA index between primary and metastatic sarcoma sites was present in half of the cases examined.
\end{abstract}


Hypoploidy is more common in canine sarcomas and hyperploid cases have less deviation of the DNA index than human sarcomas. The variation in the presence and extent of aneuploidy amongst sarcoma subtypes indicates variation in genomic instability. This study strengthens the concept of interspecies variation in the evolution of gross chromosomal aberrations during cancer development.

Keywords: aneuploidy evolution; canine; sarcomas; DNA index

\begin{abstract}
Abbreviations
Canine Kidney Cells, CKC; Chicken Red Blood Cells, CRBC; Coefficient of Variation, CV; DNA Index, DI; Flow Cytometry, FCM; Histiocytic Sarcoma, HS; Malignant Tumor of Bone, MTB; Osteosarcoma, OS; Peridiploid, PD; Soft Tissue Sarcoma, STS.
\end{abstract}

\title{
1. Introduction
}

In dogs cancer is the most common cause of non-traumatic death [1]. Several types of canine malignant neoplasms - based upon pathobiology - can serve as useful models for rare cancers in humans, including osteosarcomas (OS) and soft tissue sarcomas (STS) [2-4], both types being relatively common in the dog $[5,6]$.

As in humans, the prognosis of canine tumor patients is related to tumor location and, for malignant tumors, clinical stage [7]. A key factor in this assessment is the histological phenotype including malignancy grade $[4,8,9]$, but there is a s ignificant morphological overlap between malignant, benign, and reactive mesenchymal lesions in the human [10,11]. For both species, classification and management of mesenchymal tumors is complicated by the fact that these form a very heterogeneous group $[9,12]$. In order to improve the prognostic value of histological examination of Malignant Tumor of Bone (MTB) and of STS, several classification and grading systems have been established in humans [13], and later adopted for use in the dog [8,9], but the prognostic value of these systems for many individual tumors is limited [4,9,14]. Therefore, objective prognostic criteria are urgently needed.

Determination of the DNA-ploidy status may help to discriminate non-neoplastic or benign neoplastic lesions from (pre)malignant neoplastic conditions [15], including lesions of mesenchymal origin [16-19]. In humans, DNA-aneuploidy is more common in high-grade sarcomas than in those of low- or intermediate-grade $[16,18,20,21]$. These potential discriminatory values still need to be examined in dogs. In addition, DNA-aneuploidy has been reported to have prognostic value in several cancer types in humans [22-24]. However, in some sarcoma subtypes, this is not always the case $[16,21,25-32]$.

Besides the diagnostic value that the DNA-ploidy status can have, the DNA index (DI) distribution may help to comprehend the nature of genomic changes during tumorigenesis [33-35]. It reflects gross chromosomal changes [36] that, as already hypothesized one century ago [37], appear to play a mechanistic role in tumorigenesis, the extent of which is subject of debate [33-36,38-49]. In approximately two-thirds of human malignant solid cancers, the evolution of karyotypic 
alterations over many cell divisions until cancer clinically manifests, is thought to be reflected by the increase of total nuclear DNA content, which peaks at 1.6-fold of the normal amount [33-35,50]. In the other one-third of human cancers, the deviation of the DI is much less prominent, or cannot be discriminated from normal due to only minute or balanced chromosomal changes [15]. In general, a significant decrease in the DNA-content or DNA-hypoploidy is rare in most human solid cancers, with a few exceptions such as chondrosarcomas [51].

Loss of function of the $P 53$ pathway has been hypothesized to be one important factor in the development of aneuploid cancers [52]. As in humans, most cancers in dogs, such as thyroid and mammary carcinomas, are DNA-aneuploid, but the extent of the aberration of the DI in DNA-aneuploid cancers is less and DNA-hypoploidy more common in such carcinomas in the dog than in humans [53-55]. In fact, these observations led us to hypothesize that there is an interspecies evolutionary variation in the manifestation of DNA-aneuploidy in tumors, when comparing humans and dogs [44].

In continuation with our earlier research [53-55], we now examined the DNA-ploidy distribution pattern as determined by flow cytometry (FCM) in a series of fresh frozen samples of canine benign and malignant mesenchymal lesions. Preliminary results of this study have been presented as poster at the ESF conference (ESF Conference, Dresden, March 2010). For sarcomas, the data were compared with information on clinical stage, histological subtype and grade and the mutational status of the P53 gene, and with published data in human sarcomas. Comparison of observations in the current and earlier studies on canine neoplasms to those published on human cancers, points to interspecies variation in the driving force of aneuploidy in the development of malignant tumors.

\section{Results}

\subsection{DNA-Ploidy Status in Primary Lesions}

The arithmetical mean of the $\mathrm{CV}_{\text {of }} \mathrm{G}_{0-1}$ populations of all lesions was 3.38 (1.4-5.0). The DNA ratio of the non-neoplastic $\mathrm{G}_{0-1}$ diploid cells as compared to $\mathrm{CRBC}_{0-1}$ cells fell within the margins established in earlier studies [53,56,57]. The ploidy status as defined by FCM-base histograms (Figure 1) was normal (diploid) in all non-malignant lesions except one lipoma with a small (10\% of all nuclei) aneuploid peak (DI 1.20).

In three dogs with histiocytic sarcoma (HS), there was multi-organ involvement. To attribute a ploidy status in such cases, the DI of the largest of multiple tumors (one dog) or one out of multiple equally-sized lesions with equal DI-results was used in the categorization of primary malignancies. There were 42 aneuploid cases out of 77 primary malignant tumors (55\%), and four (5\%) PD cases. We found a significant difference in the ploidy-status of primary malignancies compared to benign neoplastic lesions $(p=0.023)$ and to non-neoplastic proliferative lesions $(p=0.0005)$. Out of all 43 aneuploid primary tumors (including one lipoma), 35 had only a single aneuploid $\mathrm{G}_{0-1}$ population named stemline (81.4\%), whereas eight (18.6\%) had multiple aneuploid stemlines. The distribution of the DIs of all stemlines in primary sarcomas is shown in Figure 2. The ploidy status did not vary $(p=0.62)$ between all STS as compared to all MTB. 
Figure 1. Example of DNA content histograms of canine tumors.
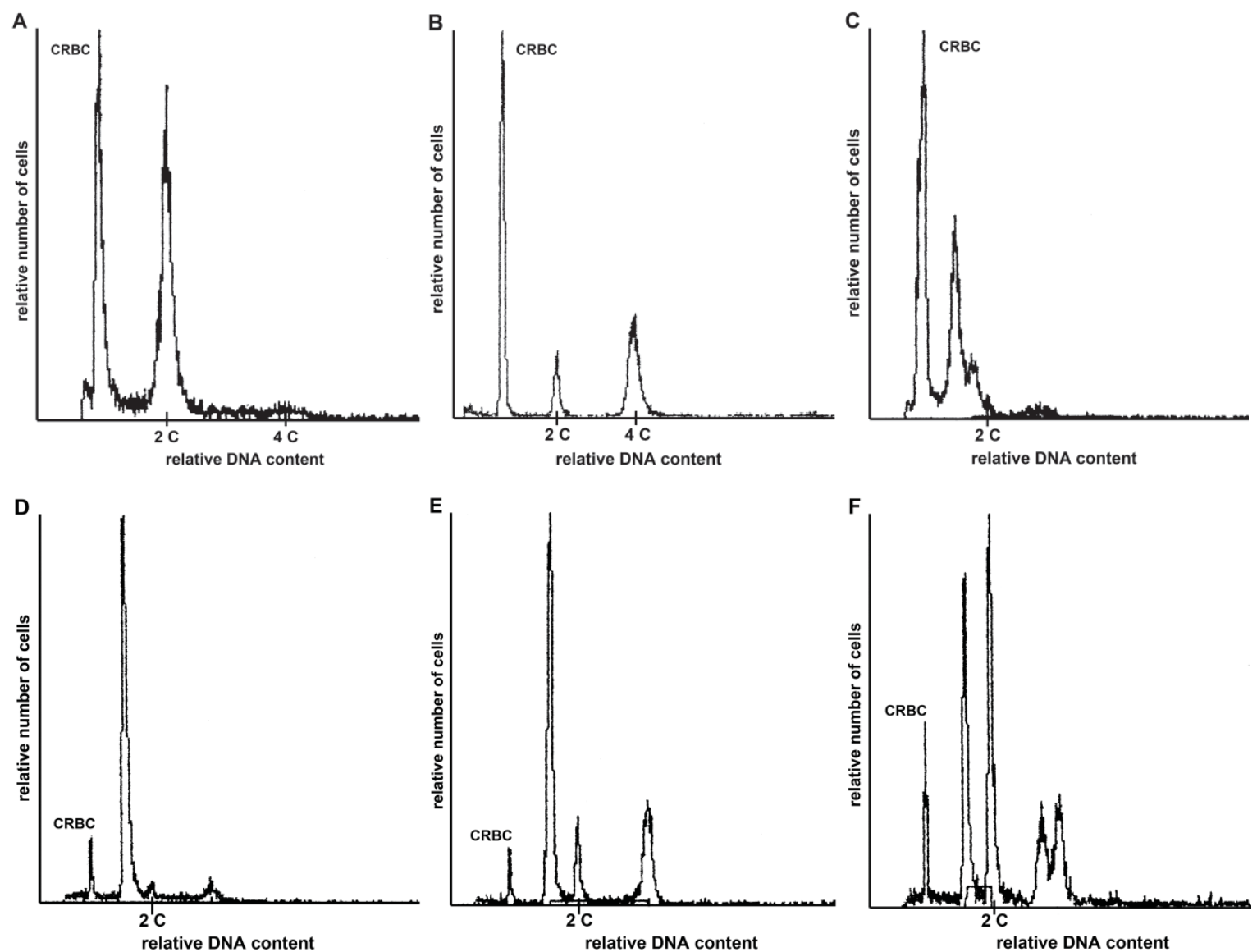

(A) Diploid tumor, osteosarcoma (OS, DI $=1.0)$; (B) Hyperploid (tetraploid) tumor, hemangiosarcoma ( $\mathrm{DI}=2.0)$; (C) Multiploid-hypoploid tumor, chondrosarcoma (DI $=0.73$ and 0.88); (D) Hypoploid primary OS (DI = 0.76); (E) Multiploid tumor (1st lung-metastases of former OS, DI $=0.77$ and 1.56); (F) Multiploid tumor (2nd lung-metastases of former OS, $\mathrm{DI}=0.79,1.43$ and 1.59$)$. Note: The stemline of the primary OS $(\mathrm{DI}=0.76)$ reappeared in the $1 \mathrm{st}$ metastases $(\mathrm{DI}=0.77)$ together with a second stemline with double the DNA content $(\mathrm{DI}=1.56)$, while in the second metastases the hypoploid stemline $(\mathrm{DI}=0.79)$ plus the polyploid stemline $(\mathrm{DI}=1.59)$ appeared together with a third stemline $(\mathrm{DI}=1.43)$.

Amongst STS, malignant peripheral nerve sheath tumors (MPNST) and leiomyosarcomas had a significantly lower rate of aneuploidy (Table 1) as compared to all other STS ( $p=0.003$ and 0.03 , respectively). In contrast, HS and synovial cell sarcomas had an increased rate of aneuploidy when compared to all other STS ( $p=0.01$ and 0.02 , respectively) or to MPNST or leiomyosarcomas $(p<0.02)$.

Twenty of the 77 primary sarcomas had hypoploid stemlines $(26 \%$, or $48 \%$ of the aneuploid cases). Amongst aneuploid sarcomas the occurrence of hypoploidy in STS (9 of 25; 36\%) and MTB (11 of $17 ; 65 \%)$ did not differ significantly $(p=0.115)$.

Noteworthy is the relative high DI w ithin the three liposarcoma cases $(2.0,2.3$ and 3.2 respectively), in relation to the general DI distribution in primary malignancies (Figure 1). There was no significant relation between ploidy status and histological malignancy grade (Table 2, grade I + II versus III in MTB: $p=1.0$, in STS: $p=0.16$, in all sarcomas: $p=0.15$ ). Note that this analysis 
excluded the three PD cases. Examination of a possible relation between ploidy-status and the p53 gene mutational status in 44 sarcomas was negative (Table 3).

Figure 2. DNA indices of stemlines present in all 77 primary malignant lesions.

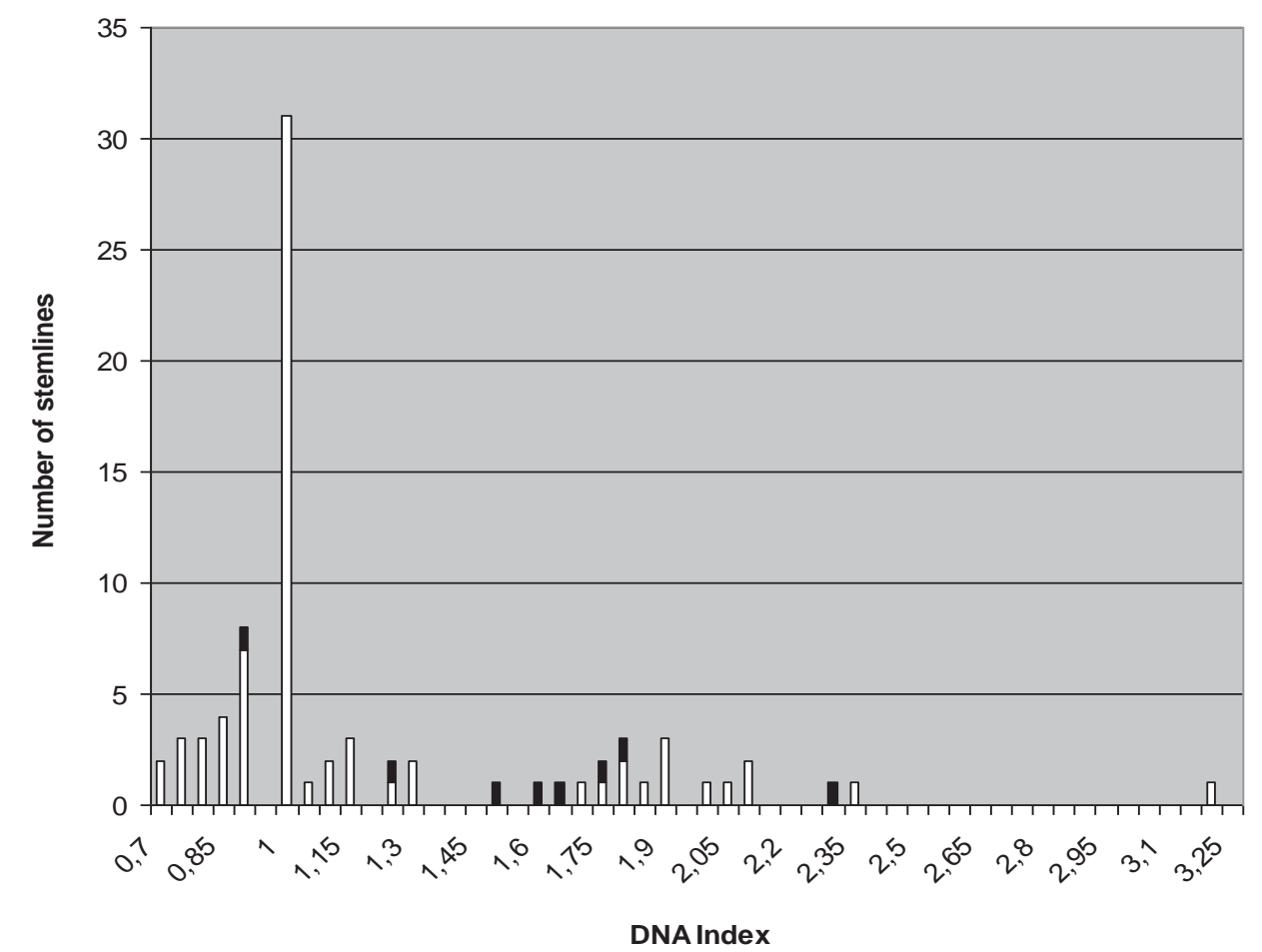

In cases in which multiple aneuploid peaks were present, both of the stemlines were represented with the stemline was the lowest DI in white and the stemline with the highest DI in black. The 4 PD sarcomas are not represented in the table.

Table 1. DNA-ploidy status in 77 sarcomas according to subtype.

\begin{tabular}{lcccc}
\hline \multicolumn{1}{c}{ Subtype } & Diploid (n) & Aneuploid (n) & Peridiploid (n) & Total (n) \\
\hline Malignant tumors of bone & & & & \\
\hline Osteosarcoma & 9 & 14 & 3 & 26 \\
Chondrosarcoma & 1 & 2 & - & 3 \\
Multilobular tumor of bone & 0 & 1 & - & 1 \\
\hline Total & $\mathbf{1 0}$ & $\mathbf{1 7}$ & $\mathbf{3}$ & $\mathbf{3 0}$ \\
\hline Soft tissue sarcomas & & & & \\
\hline Fibrosarcoma & 2 & 1 & - & 3 \\
Sarcoma-not otherwise specified & 3 & 1 & - & 4 \\
Rhabdomyosarcoma & 2 & 3 & - & 5 \\
Malignant peripheral nerve sheath tumors & 7 & 1 & - & 8 \\
Synovial sarcoma & 0 & 5 & - & 5 \\
Liposarcoma & 0 & 3 & - & 3 \\
Leiomyosarcoma & 6 & 2 & - & 8 \\
Hemangiosarcoma & 1 & 3 & - & 4 \\
Histiocytic sarcoma & 0 & 6 & 1 & 7 \\
Total & $\mathbf{2 1}$ & $\mathbf{2 5}$ & $\mathbf{1}$ & $\mathbf{4 7}$ \\
\hline
\end{tabular}


Table 2. DNA-ploidy status and histological malignancy grade in 59 sarcomas.

\begin{tabular}{|c|c|c|c|}
\hline Tumor type and grade & Diploid (n) & Peridiploid (n) & Aneuploid (n) \\
\hline \multicolumn{4}{|c|}{ Malignant tumor of bone } \\
\hline -grade I & 0 & 0 & 0 \\
\hline -grade II & 1 & 1 & 2 \\
\hline -grade III & 8 & 2 & 12 \\
\hline \multicolumn{4}{|c|}{ Soft tissue sarcoma } \\
\hline -grade I & 5 & 0 & 2 \\
\hline -grade II & 5 & 0 & 2 \\
\hline -grade III & 8 & 0 & 11 \\
\hline
\end{tabular}

Table 3. Presence of p53 mutations in sarcomas $(n=44)$ as related to the ploidy-status.

\begin{tabular}{cccc}
\hline & Diploid & Aneuploid & Peridiploid \\
\hline P53-wt & 12 & 14 & 2 \\
\hline P53- alteration & 7 & 9 & 1 \\
\hline
\end{tabular}

Note: only mutations predicted to alter the amino acid composition of the $\mathrm{p} 53$ protein were counted.

We then compared ploidy status with metastatic behavior. Only cases with macroscopic metastasis were considered, found either at first presentation (including those with postmortem after euthanasia) or during a follow up period for up to one year. PD cases $(n=4)$ and cases with less than one year follow up after tumor resection ( $n=22$, including three PD cases) were excluded. In the category of MTB only 20 dogs with OS fulfilled the criteria; all developed metastases either at first presentation $(n=2)$ or after surgical removal of the primary tumor $(n=18)$ without any influence of ploidy status (Table 4).

Table 4. The metastatic behavior of the sarcomas and the ploidy status of the primary lesion.

\begin{tabular}{ccc}
\hline \multirow{2}{*}{ Tumor group } & \multicolumn{2}{c}{ Ploidy status } \\
\cline { 2 - 3 } & Diploid & Aneuploid \\
\hline & Osteosarcomas \\
\hline -Metastases & 8 & 12 \\
-No metastases & 0 & 0 \\
\hline \multicolumn{3}{c}{ Soft tissue sarcomas } \\
\hline -Metastases & 5 & 17 \\
-No metastases & 8 & 3 \\
\hline
\end{tabular}

Note: PD cases were excluded, as well as cases that lacked information on metastatic growth or recurrence within the first year following the initial diagnosis.

When viewing STS as a group (including HS), 22 out of $34 \mathrm{~d}$ ogs had metastases at $\mathrm{f}$ irst presentation or during follow up after tumor resection. Aneuploid STS more often had metastases than diploid STS $(p=0.0092)$. The difference was no longer significant ( $p=0.067)$ after exclusion of HS. All HS cases but one (which was euthanized upon diagnosis) had metastases at first presentation. 


\subsection{Heterogeneity of Ploidy Status or DI}

In 12 dogs, we were able to compare the DI of the primary and metastatic lesions (Table 5). In six of these dogs, a significant variation in DI was noticed: The DI changed from a diploid into an aneuploid (hyperdiploid) pattern in two sarcomas and from an aneuploid (hyperdiploid) to a diploid pattern in one other. In one dog with HS, in which the largest tumor (lung) and a sternal lymph node were both analyzed, the pulmonary lesion was PD an d the nodal was low-level hypodiploid (DI 0.93). No particular significance was ascribed to this difference. In another three cases extra stemlines were identified on one location as compared to the other location(s), which in two of these cases led to the (dis)appearance of a stemline, containing twice the total DNA content.

Table 5. Comparison of the DNA index (DI) in p rimary versus metastatic lesions (Met. 1 to 4) from the same tumors.

\begin{tabular}{cccccc}
\hline Case & Primary & Met. 1 & Met. 2 & Met. 3 & Met. 4 \\
\hline Osteosarcoma $1 *$ & 2.10 & $1.10 / 2.14$ & 1.12 & & \\
Osteosarcoma 2* & 0.76 & $0.77 / 1.56$ & $0.79 / 1.43 / 1.59$ & $0.79 / 1.55$ & $0.78 / 1.55$ \\
Osteosarcoma 3 & 1.0 & 1.88 & & & \\
Osteosarcoma 4 & 1.0 & 1.0 & 1.0 & & \\
Synovial cell sarcoma * & $0.89 / 1.80$ & 1.77 & & & \\
Hemangiosarcoma * & 1.89 & 1.0 & 1.0 & & \\
Sarcoma-NOS * & 1.0 & 1.77 & & & \\
Fibrosarcoma & 1.0 & 1.0 & 1.0 & & \\
Malignant Peripheral & 1.0 & 1.0 & & & \\
Nerve Sheet Tumor & & & & & \\
Synovial Cell Sarcoma & 0.72 & 0.72 & & & \\
Histiocytic Sarcoma & PD & 0.93 & & & \\
Liposarcoma & 2.03 & 1.97 & & & \\
\hline
\end{tabular}

Note: The variations in the DI became visible as peaks with $>10 \%$ of the total cell population analyzed. Met: Metastasis, NOS: Not otherwise specified. Marked with an asterisk $(*)$ are six dogs that had significant variation in DI comparing primary-and metastatic lesion.

\section{Discussion}

Our study indicates that the existence of aneuploidy in canine mesenchymal proliferative lesions is suggestive but no proof of a malignancy, in accordance with observations in humans [16,58-60], although it must be recognized that a diploid status does not rule out malignancy. This finding may lead to additional cytological/histological testing of canine mesenchymal lesions in which the results of routine diagnosis remain ambiguous.

For sarcomas in humans, a relationship between histological grade of malignancy and ploidy pattern has often been reported $[16,20,59,61]$, but no such relationship was evident in our study. In part, this may be related to the relatively high frequency of hypoploid cancers, and the related presence of smaller nuclei, which might be judged lower grade by pathologists.

As in earlier studies in mammary malignant tumors in dogs $[22,53,55]$ and in humans $[22,23,30]$, we were also able to demonstrate a relation between ploidy status and risk of metastases in STS. Many studies in humans $[28,30,31,62]$ reported a s imilar correlation, although this is not 
universal [58]. Striking was the absolute lack of a relation between ploidy status and risk of metastasis in OS, which is in contrast to OS in humans [32,63]. In diploid cases within particular types of cancer more detailed cytogenetic analyses are required to discern those changes that predict metastatic behavior $[62,64]$.

Consistent with early research [65-67], clear similarities were found with respect to aneuploidy occurrence amongst STS (53\%) and MTB (57\%) when compared to such sarcomas in humans $[50,58,60]$. For the subtypes of MPNST and leiomyosarcoma a diploid status is common in both dogs (as seen in our study) and humans [68]. However, in most human sarcomas hypodiploidy is rare [10,50], with the highest reported rate being $11 \%$ [58], while chondrosarcomas are an exception since they are frequently hypoploid [69]. We observed a remarkable overall hypoploidy incidence of $26 \%$ ( $48 \%$ of aneuploid cases). One other study in canine OS reported a somewhat lower figure [67]. In addition, both studies indicate that for many subtypes of canine sarcomas the net increase in DNA content in hyperploid cases is modest when compared to their human counterparts $[16,58,70]$ albeit that some sarcoma types, such as the three liposarcomas in our study, form an exception, since these were all (hyper)tetraploid.

Since similar observations have been made in other types of cancer in the dog such as thyroid and mammary carcinomas and malignant lymphomas $[53,54,56,57]$, it seems that the dog is particularly prone to the development of aneuploid tumors associated with either chromosome loss or low number chromosome gain, and less frequently to hypertriploid tumors that are common in humans $[33,34,40,50]$. This feature seems at variance with ploidy evolution patterns described in humans. In humans, this evolution can be divided in s tages with early on the development of tetraploidy which is followed by chromosome loss and later on leads to a major proportion (amongst aneuploid cases) of cancers manifesting an increase in DI of approximately 1.6-1.7 [33-35,40,45,50]. Still, in a few sarcomas of the current study and in mammary carcinomas [53,65] tetraploidization (in the primary cancer) followed by an appearance of hypotetraploid stemlines (in its metastases) has been observed. A possible explanation for this difference in ploidy evolution could be the presence of a more powerful defense mechanism in humans against the tumorigenic effects of hypodiploidy or low level hyperploidy [33,71]. While for many human cancers a greater destabilization over multiple phases of destabilizing events seems necessary to arrive at a fully malignant state, such state may be reached with less destabilization in the dog, as hypothesized earlier [44]. How some cancers can reach at a fully malignant state while remaining DNA-diploid is uncertain. More subtle and sometimes balanced chromosomal gains and losses have been observed by cytogenetic analysis in some such human cancers [34] including sarcomas [19,68], and also in canine sarcomas [72,73] and carcinomas [74]. In other human diploid cancers however, structural chromosomal abnormalities are absent, and the transformation to malignancy seems to be driven by defects in DNA repair pathways leading to microsatellite instability, with microsatellite instability and aneuploidy being mutually exclusive phenomena [46].

It must be recognized that the karyotypic alterations that occur in dog cancer are to some extent at variance with those described for human cancers. The autosomes in normal dog cells are acrocentric/telocentric, and changes in canine cancers often concern centric fusions [74-77] albeit that alterations such as trisomies or monosomies and translocations are also frequent $[72,75,78,79]$ as well as smaller structural abnormalities that have been detected with comparative genomic 
hybridization studies [73,79-81] in the past few years. Still, as mentioned in an extensive review by Breen and Thomas, "tumors of the same histological types in both species present with equivalent cytogenetic lesions" [82].

In cancers that harbour significant chromosomal alterations, even if not recognized as DNA-aneuploid, the cause underlying this chromosomal instability has been subject of study and aberrations in many pathways, in particular those related to s ister chromatid cohesion and segregation have been suggested as possible causes [46,47,49,83-85]. Contrasting views exist as to whether loss of $P 53$ function is essential for the development of aneuploidy [52,86-88]. In the current study, no relation between P53 mutations and DNA-ploidy status was found. Although it must be recognized that analysis for P53 mutations concerns only part of the many elements involved in the P53 pathway, our results are in clear contrast with a study in human sarcomas that demonstrated a relation between the presence of P53 mutations and DNA-aneuploidy [61].

Regarding whether and how aneuploidy may be essential for the development of cancer [38] it is essential to agree on the definition of malignancy. Most oncologists agree that proof of metastatic potential is not the only prerequisite for a tumor to be considered malignant and that tumors with extensive infiltrative destructive growth and low or late risk of metastases such as leiomyosarcomas and MPNSTs in the dog should also be regarded as malignant [7]. In our study, these sarcomas were only rarely found to be aneuploid. There is no doubt that many malignancies with full malignant potential are aneuploid, reflecting gross quantitative genomic destabilization. Several major disturbances must have taken place in order to overcome restraints, which are-in part tissue specific - against malignant transformation. Other cancers, such as the diploid OS in the current study, can reach this state of progression with much less genome destabilization. Functional changes that may allow malignant behavior in such cancers may include P53 inactivation or MDM2 amplification $[89,90]$ as well as many other genetic alterations. Such changes may be called high hierarchy changes. This is opposite to many more subtle low hierarchy changes that can accumulate during progressive genomic destabilization and, by chance or number, can also disrupt crucial control mechanisms. To some extent and for some types of sarcomas (such as MPNST and leiomyosarcoma), a diploid status is then related to low metastatic potential. For OS in the dog a highly metastatic state can be either reached by appearance of a relatively low number of high hierarchy genetic changes, and low level of genomic destabilization in the form of chromosomal instability, while in others a high level of genomic destabilization, involving more lower hierarchy changes, may lead to this state.

The debate on gene mutation versus aneuploidization as cause of cancer cannot be resolved, since both phenomena may be linked and cooperative in tumor development $[36,48]$.

Once a malignant tumor has developed, the same DI can often be found in both the primary and the metastatic lesion. Sometimes, haploidization or duplication of such stemlines occurs, with variation between metastases. In other tumors in the current and earlier studies $[53,65,67]$ stemlines were found which really looked like an unrelated clone, able to pr opagate as independent subpopulation, as hypothesized previously [91]. 


\section{Experimental Section}

\subsection{Animals}

Lesions from pet-dogs of various breeds, sex and age were selected for this study. Samples with suspicion of a neoplastic origin were obtained, with informed consent from the owner, by biopsy or surgical excision as part of normal diagnostic procedure or treatment, or at postmortem immediately following euthanasia. After previous studies by our research group on DNA-ploidy* of carcinomas [53,54,56] and of malignant lymphomas [57], the focus was on proliferative, inflammatory or neoplastic lesions of the mesenchyme.

All samples had been characterized by routine histopathology by different veterinary pathologists and were centrally reviewed for the current study. The ploidy assessment was not possible in three soft tissue sarcomas, one inflammatory lesion and two benign lesions, probably due to the amount of debris present. These samples were excluded from further analyses. Remaining samples, from 95 dogs, included non-neoplastic, proliferative, inflammatory lesions $(\mathrm{n}=10)$, benign tumors $(\mathrm{n}=8$ : lipoma 4), fibrous dysplasia of bone, osteochondroma, leiomyoma, fibrous epulis), and 77 malignancies (including one local recurrence; detailed description is listed in Table 2), from 77 dogs. From 12 of these 77 dogs both primary and recurrent specimens were available for examination.

Prior to collection of the tissue samples, none of the patients had received any cytostatic or radiotherapeutic treatment. Data on the occurrence of metastasis at first presentation or for up to one year after surgery were collected from the hospital records. Where in the following text the word ploidy is used as related to the current study, it indicates DNA-ploidy.

\subsection{Preparation of Samples}

Upon surgery or euthanasia, tissue samples were immediately placed in melting ice. From areas of the mass not hampering full histological evaluation, thus avoiding planes of resection, blocks of approximately $5 \mathrm{~mm}^{3}$ were cut, trimmed of fat and necrotic parts, snap-frozen in liquid nitrogen and stored at $-70{ }^{\circ} \mathrm{C}$ until further analysis. The tissue samples were thawed only once, directly prior to FCM. Adjacent blocks including the resection planes were fixed in neutral phosphate-buffered $10 \%$ formalin for histological examination.

\subsection{Histological Examination}

The tissues were routinely processed; paraffin embedded and cut to 4-6 $\mu \mathrm{m}$ sections that were stained with hematoxylin-eosin (H\&E). Cases were re-evaluated by board-certified veterinary pathologist (CS, GCMG) and categorized according to the WHO classification for tumors in domestic animals (WHO 1994, 1998). STS were grouped by their entity, including additional immunohistochemistry if deemed appropriate [89,92,93]. Most cases were reviewed, except for eleven due to sample loss, for which the original diagnoses were used. With the exclusion of histiocytic sarcomas (HS), a proper grading system is available for STS [8,9] as well as for OS [4]. This was applied for the current series, except for ten cases that could not be graded leaving a total of 59 cases. 


\subsection{Flow Cytometry Analysis of Nuclear DNA Content}

FCM of nuclear DNA content was performed as previously described [53,57] using the detergent-trypsin procedure of Vindolov et al. [94]. Isolated nuclei from all lesions were stained with propidium iodide (Sigma Chemical Co., St. Louis, MO, USA). All neoplastic samples were required to contain an adequate proportion of at least $20 \%$ tumor cells and the cell yield of all specimens had to permit analysis of $\geq 5,000$ cells in each assay. As external standard, Chicken Red Blood Cells (CRBC) was added to determine the position of the $\mathrm{G}_{0-1}$ peak(s). In some samples two $\mathrm{G}_{0-1}$ peaks were discerned of which the DNA content both fell within the range of the ratio of the DNA content of normal dog diploid cells compared to that of CRBC. Then a second analysis was done with and without addition of Canine Kidney Cells (CKC) as a diploid standard. The increase in height of one of those peaks upon addition of CKC identified this peak as diploid and the other as aneuploid. The ploidy analysis itself was performed using the FAC Scan 3 flow cytometer (Becton Dickinson, Mountain View, CA, USA). The device is able to detect changes above $5 \%$ in nuclear DNA content. The propidium iodide fluorescence was excited at $488 \mathrm{~nm}$ and measured at $585 \mathrm{~nm}$. Since past research has indicated that, by measuring only a single tissue block, true aneuploid stemlines can sometimes be missed [58], many lesions - in particular large-sized ones - were analyzed using two or more tissue blocks. In 11 primary tumors of larger size $(>3 \mathrm{~cm})$ more than one tissue block was analyzed to as sess possible intra-tumor heterogeneity. When a difference was found between the separate analyses, the measurement was repeated several times with and without the external CKC reference standard.

\subsection{DNA-Ploidy Assessment}

The DI is defined as the ratio of the modal channel number of the $\mathrm{G}_{0-1}$ peak of the (neoplastic) cell population in relation to the modal channel number of the $\mathrm{G}_{0-1}$ fraction of diploid cells. The latter was recognized by its relative position to the $\mathrm{G}_{0-1}$ peak of $\mathrm{CRBC}$ and by is position to the normal CKC (normal dog reference) peak [53]. A sample was considered diploid if the DI was between 0.95 and 1.05 and aneuploid if there was a distinct $\mathrm{G}_{0-1}$ population with $\mathrm{DI}<0.95$ or $>1.05$. A peak with a DI of 1.90-2.10 was considered to be a tetraploid $\mathrm{G}_{0-1}$ peak if it contained $>20 \%$ of the total number of analyzed cells and if a G2-M peak was also present. Aneuploid stemlines were subdivided into hypoploid (DI $<1.0$ ) or hyperploid (DI $>1.0$ ) When more than one aneuploid $\mathrm{G}_{0-1}$ population was present the sample was classified as multiploid. For all samples, the coefficient of variation (CV) for the $\mathrm{G}_{0-1}$ peak was measured. A CV above 5\% was considered suspicious for the presence of an aneuploid peak within a diploid population. Samples with peaks with a CV $>5.1 \%$ were re-analyzed with and without the CKC reference cells, and if this led to a clear recognition of two separate peaks, it was considered aneuploid. However, if under these conditions a single $\mathrm{G}_{0-1}$ peak with $\mathrm{CV}>5.1 \%$ remained, the sample was considered neither diploid nor aneuploid but peridiploid (PD). All non-diploid, non-PD lesions were grouped together as aneuploid, including those with heterogeneity in ploidy status within the primary site. 
In two earlier studies, part of the sarcomas had been analyzed for the presence of p53 mutations. For STS the p53 gene was analyzed for exons IV-VIII, covering most of the mutations occurring in cancer [89]; for MTB exons I-X were examined [90]. Eight STS had been analyzed during the study of MTB that have not yet been published. The results of both investigations including a tot al of 44 sarcomas were used for a comparison with the ploidy-status. Only mutations predicted to affect the amino-acid sequence of the p53 gene [95] including point mutations, deletions and insertions, and mutations of the splice site were counted.

\subsection{Statistical Analysis}

Differences in frequency distribution in data groups were analyzed using Fishers exact test. The level of significance was set at 0.05 . PD samples were excluded in the statistical analysis comparing diploid and aneuploid status.

\section{Conclusions}

In conclusion, aneuploidy is frequent in various canine malignant lesions, with prominent variation amongst the different sarcoma types. The overall frequency of aneuploidy is largely in accordance with findings in humans [50]. However, the high frequency of tumors with hypoploidy and low-level hyperploidy in various canine cancers [53,54,57] when compared to human malignancies including sarcomas [50], is striking. In human solid cancers hypoploidy is rare and the majority of cases are hypertriploid thought to arise from a tetraploid intermediate $(33-35,46,50)$. Our findings therefore are suggestive of interspecies variation in aneuploidy evolution and genomic destabilization during carcinogenesis.

\section{Acknowledgments}

This study was partly funded by the European Commission (LUPA-GA-201370). The authors wish to thank N.J. Kuipers-Dijkshoorn for expert flow cytometric analyses and K. Thway for her useful comments. J. Joles is thanked for reading and correcting the manuscript.

\section{Conflicts of Interest}

The authors declare no conflict of interests.

\section{References}

1. Fleming, J.M.; Creevy, K.E.; Promislow, D.E. Mortality in North American Dogs from 1984 to 2004: A n Investigation into Age-, Size-, and B reed-Related Causes of Death. J. Vet. Intern. Med. 2011, 25, 187-198.

2. Paoloni, M.; Khanna, C. Translation of New Cancer Treatments from Pet Dogs to Humans. Nat. Rev. Cancer 2008, 8, 147-156. 
3. Paoloni, M.; Davis, S.; Lana, S.; Withrow, S.; Sangiorgi, L.; Picci, P.; Hewitt, S.; Triche, T.; Meltzer, P.; Khanna, C. Canine Tumor Cross-Species Genomics Uncovers Targets Linked to Osteosarcoma Progression. BMC Genomics 2009, 10, 625.

4. Kirpensteijn, J.; Kik, M.; Rutteman, G.R.; Teske, E. Prognostic Significance of a New Histologic Grading System for Canine Osteosarcoma. Vet. Pathol. 2002, 39, 240-246.

5. Dobson, J.M.; Samuel, S.; Milstein, H.; Rogers, K.; Wood, J.L. Canine Neoplasia in the UK: Estimates of Incidence Rates from a Population of Insured Dogs. J. Small Anim. Pract. 2002, 43, 240-246.

6. Rosenberger, J.A.; Pablo, N.V.; Crawford, P.C. Prevalence of and Intrinsic Risk Factors for Appendicular Osteosarcoma in Dogs: 179 Cases (1996-2005). J. Am. Vet. Med. Assoc. 2007, 231, 1076-1080.

7. Withrow, S.J.; Vail, D. Small Animal Clinical Oncology; 4th ed.; Saunders: St. Louis, MO, USA, 2007.

8. Kuntz, C.A.; Dernell, W.S.; Powers, B.E.; Devitt, C.; Straw, R.C.; Withrow, S.J. Prognostic Factors for Surgical Treatment of Soft-Tissue Sarcomas in Dogs: 75 C ases (1986-1996). J. Am. Vet. Med. Assoc. 1997, 211, 1147-1151.

9. Dennis, M.M.; McSporran, K.D.; Bacon, N.J.; Schulman, F.Y.; Foster, R.A.; Powers, B.E. Prognostic Factors for Cutaneous and Subcutaneous Soft Tissue Sarcomas in Dogs. Vet. Pathol. 2011, 48, 73-84.

10. Xiang, J.H.; Spanier, S.S.; Benson, N.A.; Braylan, R.C. Flow Cytometric Analysis of DNA in Bone and Soft-Tissue Tumors using Nuclear Suspensions. Cancer 1987, 59, 1951-1958.

11. Rosenberg, A.E. Pseudosarcomas of Soft Tissue. Arch. Pathol. Lab. Med. 2008, 132, 579-586.

12. Thway, K. Pathology of Soft Tissue Sarcomas. Clin. Oncol. 2009, 21, 695-705.

13. Trojani, M.; Contesso, G.; Coindre, J.M.; Rouesse, J.; Bui, N.B.; de Mascarel, A.; Goussot, J.F.; David, M.; Bonichon, F.; Lagarde, C. Soft-Tissue Sarcomas of Adults; Study of Pathological Prognostic Variables and Definition of a Histopathological Grading System. Int. J. Cancer 1984, 33, 37-42.

14. Jones, N.B.; Iwenofu, H.; Scharschmidt, T.; Kraybill, W. Prognostic Factors and Staging for Soft Tissue Sarcomas: An Update. Surg. Oncol. Clin. N. Am. 2012, 21, 187-200.

15. Barlogie, B.; Raber, M.N.; Schumann, J.; Johnson, T.S.; Drewinko, B.; Swartzendruber, D.E.; Gohde, W.; Andreeff, M.; Freireich, E.J. Flow Cytometry in Clinical Cancer Research. Cancer Res. 1983, 43, 3982-3997.

16. Kreicbergs, A.; Tribukait, B.; Willems, J.; Bauer, H.C. DNA Flow Analysis of Soft Tissue Tumors. Cancer 1987, 59, 128-133.

17. Kroese, M.C.; Rutgers, D.H.; Wils, I.S.; van Unnik, J.A.; Roholl, P.J. The Relevance of the DNA Index and Proliferation Rate in the Grading of Benign and Malignant Soft Tissue Tumors. Cancer 1990, 65, 1782-1788.

18. Van den Berg, E.; van Oven, M.W.; de Jong, B.; Dam, A.; Wiersema, J.; Dijkhuizen, T.; Hoekstra, H.J.; Molenaar, W.M. Comparison of Cytogenetic Abnormalities and Deoxyribonucleic Acid Ploidy of Benign, Borderline Malignant, and Different Grades of Malignant Soft Tissue Tumors. Lab. Invest. 1994, 70, 307-313. 
19. Mohamed, A.N.; Zalupski, M.M.; Ryan, J.R.; Koppitch, F.; Balcerzak, S.; Kempf, R.; Wolman, S.R. Cytogenetic Aberrations and DNA Ploidy in Soft Tissue Sarcoma. A Southwest Oncology Group Study. Cancer Genet. Cytogenet. 1997, 99, 45-53.

20. Yanong, W.; Daren, S.; Zhenzhou, S.; Renyuan, Z.; Shouye, L. Investigation of Relationships between KI-67 Score, DNA Index, and Histologic Grade in Soft Tissue Sarcomas. Chin. J. Cancer Res. 1988, 8, 55-59.

21. Plaat, B.E.; Muntinghe, F.L.; Molenaar, W.M.; Hoekstra, H.J.; Bosveld, H.E.; Dam, A.; Dijkhuizen, T.; van den Berg, E. Clinical Outcome of Patients with Previously Untreated Soft Tissue Sarcomas in Relation to Tumor Grade, DNA Ploidy and Karyotype. Int. J. Cancer 1997, 74, 396-402.

22. Friedlander, M.L.; Hedley, D.W.; Taylor, I.W. Clinical and Biological Significance of Aneuploidy in Human Tumours. J. Clin. Pathol. 1984, 37, 961-974.

23. Merkel, D.E.; McGuire, W.L. Ploidy, Proliferative Activity and Prognosis. DNA Flow Cytometry of Solid Tumors. Cancer 1990, 65, 1194-1205.

24. Kildal, W.; Abeler, V.M.; Kristensen, G.B.; Jenstad, M.; Thoresen, S.O.; Danielsen, H.E. The Prognostic Value of DNA Ploidy in a Total Population of Uterine Sarcomas. Ann. Oncol. 2009, 20, 1037-1041.

25. Lopes, J.M.; Hannisdal, E.; Bjerkehagen, B.; Bruland, O.S.; Danielsen, H.E.; Pettersen, E.O.; Sobrinho-Simoes, M.; Nesland, J.M. Synovial Sarcoma. Evaluation of Prognosis with Emphasis on the Study of DNA Ploidy and Proliferation (PCNA and Ki-67) Markers. Anal. Cell. Pathol. 1998, 16, 45-62.

26. Van De L uijtgaarden, A.C.; van der Graaf, W.T.; Otte-Holler, I.; Schreuder, H.W.; Versleijen-Jonkers, Y.M.; Slootweg, P.J. Targeted Therapy for Ewing's Sarcoma: Significance of Heterogeneity. Anticancer Res. 2010, 30, 3715-3719.

27. Chew, I.; Oliva, E. Endometrial Stromal Sarcomas: A Review of Potential Prognostic Factors. Adv. Anat. Pathol. 2010, 17, 113-121.

28. Dreinhofer, K.E.; Baldetorp, B.; Akerman, M.; Ferno, M.; Rydholm, A.; Gustafson, P. DNA Ploidy in Soft Tissue Sarcoma: Comparison of Flow and Image Cytometry with Clinical Follow-Up in 93 Patients. Cytometry 2002, 50, 19-24.

29. Niggli, F.K.; Powell, J.E.; Parkes, S.E.; Ward, K.; Raafat, F.; Mann, J.R.; Stevens, M.C. DNA Ploidy and Proliferative Activity (S-Phase) in Childhood Soft-Tissue Sarcomas: Their Value as Prognostic Indicators. Br. J. Cancer 1994, 69, 1106-1110.

30. Samur, M.; Pamir, A.; Akbulut, H.; Erekul, S.; Saglik, Y.; Yildiz, Y.; Dincol, D.; Icli, F. The Clinical Value of Flow Cytometric DNA Content Analysis in Patients with Soft Tissue Sarcomas. Sarcoma 1999, 3, 171-175.

31. Bauer, H.C.; Kreicbergs, A.; Tribukait, B. DNA Content Prognostic in Soft Tissue Sarcoma. 102 Patients Followed for 1-10 Years. Acta Orthop. Scand. 1991, 62, 187-194.

32. Mankin, H.J.; Gebhardt, M.C.; Springfield, D.S.; Litwak, G.J.; Kusazaki, K.; Rosenberg, A.E. Flow Cytometric Studies of Human Osteosarcoma. Clin. Orthop. Relat. Res. 1991, 270, $169-180$.

33. Giaretti, W. A Model on the Origin and Evolution of DNA Aneuploidy. Int. J. Oncol. 1993, 2, $165-171$. 
34. Shackney, S.E.; Smith, C.A.; Miller, B.W.; Burholt, D.R.; Murtha, K.; Giles, H.R.; Ketterer, D.M.; Pollice, A.A. Model for the Genetic Evolution of Human Solid Tumors. Cancer Res. 1989, 49, 3344-3354.

35. Dutrillaux, B.; Gerbault-Seureau, M.; Remvikos, Y.; Zafrani, B.; Prieur, M. Breast Cancer Genetic Evolution: I. Data from Cytogenetics and DNA Content. Breast Cancer Res. Treat. 1991, 19, 245-255.

36. Gordon, D.J.; Resio, B.; Pellman, D. Causes and Consequences of Aneuploidy in Cancer. Nat. Rev. Genet. 2012, 13, 189-203.

37. Boveri, T. Zur Frage Der Entstehung Maligner Tumoren; Fischer: Jena, Germany, 1914.

38. Duesberg, P.; Rasnick, D.; Li, R.; Winters, L.; Rausch, C.; Hehlmann, R. How Aneuploidy may Cause Cancer and Genetic Instability. Anticancer Res. 1999, 19, 4887-4906.

39. Kolodner, R.D.; Cleveland, D.W.; Putnam, C.D. Cancer. Aneuploidy Drives a Mutator Phenotype in Cancer. Science 2011, 333, 942-943.

40. Barrett, M.T.; Pritchard, D.; Palanca-Wessels, C.; Anderson, J.; Reid, B.J.; Rabinovitch, P.S. Molecular Phenotype of Spontaneously Arising 4N (G2-Tetraploid) Intermediates of Neoplastic Progression in Barrett's Esophagus. Cancer Res. 2003, 63, 4211-4217.

41. Nowell, P.C.; Croce, C.M. Chromosomes, Genes, and Cancer. Am. J. Pathol. 1986, 125, 7-15.

42. Lengauer, C.; Kinzler, K.W.; Vogelstein, B. Genetic Instabilities in Human Cancers. Nature 1998, 396, 643-649.

43. Holland, A.J.; Cleveland, D.W. Losing Balance: The Origin and Impact of Aneuploidy in Cancer. EMBO Rep. 2012, 13, 501-514.

44. Cornelisse, C.J.; Rutteman, G.R.; Kuipers-Dijkshoorn, N.J.; Hellmen, E. The Difference in DNA Ploidy Pattern between some Canine and Human Neoplasms Appears to be Genuine and a Reflection of Dissimilarities in DNA Aneuploidy Evolution. Anticancer Res. 1994, 14, $1599-1601$.

45. Merlo, L.M.; Wang, L.S.; Pepper, J.W.; Rabinovitch, P.S.; Maley, C.C. Polyploidy, Aneuploidy and the Evolution of Cancer. Adv. Exp. Med. Biol. 2010, 676, 1-13.

46. Rajagopalan, H.; Lengauer, C. Aneuploidy and Cancer. Nature 2004, 432, 338-341.

47. Yen, T.J.; Kao, G.D. Mitotic Checkpoint, Aneuploidy and Cancer. Adv. Exp. Med. Biol. 2005, 570, 477-499.

48. Pihan, G.; Doxsey, S.J. Mutations and Aneuploidy: Co-Conspirators in Cancer? Cancer Cell 2003, 4, 89-94.

49. Holland, A.J.; Cleveland, D.W. Boveri Revisited: Chromosomal Instability, Aneuploidy and Tumorigenesis. Nat. Rev. Mol. Cell Biol. 2009, 10, 478-487.

50. Frankfurt, O.S.; Slocum, H.K.; Rustum, Y.M.; Arbuck, S.G.; Pavelic, Z.P.; Petrelli, N.; Huben, R.P.; Pontes, E.J.; Greco, W.R. Flow Cytometric Analysis of DNA Aneuploidy in Primary and Metastatic Human Solid Tumors. Cytometry 1984, 5, 71-80.

51. Olsson, L.; Paulsson, K.; Bovee, J.V.; Nord, K.H. Clonal Evolution through Loss of Chromosomes and Subsequent Polyploidization in Chondrosarcoma. PLoS One 2011, 6, e24977.

52. Thompson, S.L.; Compton, D.A. Proliferation of Aneuploid Human Cells is Limited by a p53-Dependent Mechanism. J. Cell Biol. 2010, 188, 369-381. 
53. Rutteman, G.R.; Cornelisse, C.J.; Dijkshoorn, N.J.; Poortman, J.; Misdorp, W. Flow Cytometric Analysis of DNA Ploidy in Canine Mammary Tumors. Cancer Res. 1988, 48, 3411-3417.

54. Verschueren, C.P.; Rutteman, G.R.; Kuipers-Dijkshoorn, N.J.; Sjollema, B.E.; Vos, J.H.; van Dijk, J.E.; Cornelisse, C.J. Flow-Cytometric DNA Ploidy Analysis in Primary and Metastatic Canine Thyroid Carcinomas. Anticancer Res. 1991, 11, 1755-1761.

55. Hellmen, E.; Lindgren, A.; Linell, F.; Matsson, P.; Nilsson, A. Comparison of Histology and Clinical Variables to DNA Ploidy in Canine Mammary Tumors. Vet. Pathol. 1988, 25, 219-226.

56. Perez Alenza, M.D.; Rutteman, G.R.; Kuipers-Dijkshoorn, N.J.; Pena, L.; Montoya, A.; Misdorp, W.; Cornelisse, C.J. DNA Flow Cytometry of Canine Mammary Tumours: The Relationship of DNA Ploidy and S-Phase Fraction to Clinical and Histological Features. Res. Vet. Sci. 1995, 58, 238-243.

57. Teske, E.; Rutteman, G.R.; Kuipers-Dijkshoorn, N.J.; van Dierendonck, J.H.; van Heerde, P.; Cornelisse, C.J. DNA Ploidy and Cell Kinetic Characteristics in Canine Non-Hodgkin's Lymphoma. Exp. Hematol. 1993, 21, 579-584.

58. Collin, F.; Chassevent, A.; Bonichon, F.; Bertrand, G.; Terrier, P.; Coindra, J.M. Flow Cytometric DNA Content Analysis of $185 \mathrm{~S}$ oft Tissue Neoplasms Indicates that S-Phase Fraction is a Prognostic Factor for Sarcomas. French Federation of Cancer Centers (FNCLCC) Sarcoma Group. Cancer 1997, 79, 2371-2379.

59. El-Naggar, A.K.; Hurr, K.; Tu, Z.N.; Teague, K.; Raymond, K.A.; Ayala, A.G.; Murray, J. DNA and RNA Content Analysis by Flow Cytometry in the Pathobiologic Assessment of Bone Tumors. Cytometry 1995, 19, 256-262.

60. Helio, H.; Karaharju, E.; Nordling, S. Flow Cytometric Determination of DNA Content in Malignant and Benign Bone Tumours. Cytometry 1985, 6, 165-171.

61. Schneider-Stock, R.; Radig, K.; Oda, Y.; Mellin, W.; Rys, J.; Niezabitowski, A.; Roessner, A. P53 Gene Mutations in Soft-Tissue Sarcomas-Correlations with p53 Immunohistochemistry and DNA Ploidy. J. Cancer Res. Clin. Oncol. 1997, 123, 211-218.

62. Balogh, Z.; Szemlaky, Z.; Szendroi, M.; Antal, I.; Papai, Z.; Fonyad, L.; Papp, G.; Changchien, Y.C.; Sapi, Z. Correlation between DNA Ploidy, Metaphase High-Resolution Comparative Genomic Hybridization Results and Clinical Outcome of Synovial Sarcoma. Diagn. Pathol. 2011, 6, 107.

63. Kreicbergs, A.; Boquist, L.; Borssen, B.; Larsson, S.E. Prognostic Factors in Chondrosarcoma: A Comparative Study of Cellular DNA Content and Clinicopathologic Features. Cancer 1982, 50, 577-583.

64. Mertens, F.; Stromberg, U.; Mandahl, N.; Dal Cin, P.; de Wever, I.; Fletcher, C.D.; Mitelman, F.; Rosai, J.; Rydholm, A.; Sciot, R.; et al. Prognostically Important Chromosomal Aberrations in Soft Tissue Sarcomas: A Report of the Chromosomes and Morphology (CHAMP) Study Group. Cancer Res. 2002, 62, 3980-3984.

65. Hellmen, E.; Svensson, S. Progression of Canine Mammary Tumours as Reflected by DNA Ploidy in Primary Tumours and their Metastases. J. Comp. Pathol. 1995, 113, 327-342. 
66. Johnson, T.S.; Raju, M.R.; Giltinan, R.K.; Gillette, E.L. Ploidy and DNA Distribution Analysis of Spontaneous Dog Tumors by Flow Cytometry. Cancer Res. 1981, 41, 3005-3009.

67. Fox, M.H.; Armstrong, L.W.; Withrow, S.J.; Powers, B.E.; LaRue, S.M.; Straw, R.C.; Gillette, E.L. Comparison of DNA Aneuploidy of Primary and Metastatic Spontaneous Canine Osteosarcomas. Cancer Res. 1990, 50, 6176-6178.

68. Mertens, F.; Dal Cin, P.; de Wever, I.; Fletcher, C.D.; Mandahl, N.; Mitelman, F.; Rosai, J.; Rydholm, A.; Sciot, R.; Tallini, G.; et al. Cytogenetic Characterization of Peripheral Nerve Sheath Tumours: A Report of the CHAMP Study Group. J. Pathol. 2000, 190, 31-38.

69. Feitz, W.F.; Karthaus, H.F.; Beck, H.L.; Romijn, C.; van der Meyden, A.P.; Debruyne, F.M.; Vooijs, G.P.; Ramaekers, F.C. Tissue-Specific Markers in Flow Cytometry of Urological Cancers. II. Cytokeratin and Vimentin in Renal-Cell Tumors. Int. J. Cancer 1986, 37, 201-207.

70. Kilpatrick, S.E.; Teot, L.A.; Geisinger, K.R.; Martin, P.L.; Shumate, D.K.; Zbieranski, N.; Russell, G.B.; Fletcher, C.D. Relationship of DNA Ploidy to H istology and Prognosis in Rhabdomyosarcoma. Comparison of Flow Cytometry and Image Analysis. Cancer 1994, 74, $3227-3233$.

71. Thompson, S.L.; Bakhoum, S.F.; Compton, D.A. Mechanisms of Chromosomal Instability. Curr. Biol. 2010, 20, R285-R295.

72. Milne, B.S.; Hoather, T.; O'Brien, P.C.; Yang, F.; Ferguson-Smith, M.A.; Dobson, J.; Sargan, D. Karyotype of Canine Soft Tissue Sarcomas: A Multi-Colour, Multi-Species Approach to Canine Chromosome Painting. Chromosome Res. 2004, 12, 825-835.

73. Aguirre-Hernandez, J.; Milne, B.S.; Queen, C.; O'Brien, P.C.; Hoather, T.; Haugland, S.; Ferguson-Smith, M.A.; Dobson, J.M.; Sargan, D.R. Disruption of Chromosome 11 in Canine Fibrosarcomas Highlights an Unusual Variability of CDKN2B in Dogs. BMC Vet. Res. 2009, 5, 27.

74. Tap, O.T.; Rutteman, G.R.; Zijlstra, C.; de Haan, N.A.; Bosma, A.A. Analysis of Chromosome Aberrations in a Mammary Carcinoma Cell Line from a Dog by using Canine Painting Probes. Cytogenet. Cell Genet. 1998, 82, 75-79.

75. Mayr, B.; Kramberger-Kaplan, E.; Loupal, G.; Schleger, W. Analysis of Complex Cytogenetic Alterations in Three Canine Mammary Sarcomas. Res. Vet. Sci. 1992, 53, 205-211.

76. Maeda, J.; Yurkon, C.R.; Fujisawa, H.; Kaneko, M.; Genet, S.C.; Roybal, E.J.; Rota, G.W.; Saffer, E.R.; Rose, B.J.; Hanneman, W.H.; et al. Genomic Instability and Telomere Fusion of Canine Osteosarcoma Cells. PLoS One 2012, 7, e43355.

77. Mellink, C.H.; Bosma, A.A.; Rutteman, G.R. Cytogenetic Analysis of Cell Lines Derived from Metastases of a Mammary Carcinoma in a Dog. Anticancer Res. 1989, 9, 1241-1244.

78. Reimann, N.; Nolte, I.; Bartnitzke, S.; Bullerdiek, J. Re: Sit, DNA, Sit: Cancer Genetics Going to the Dogs. J. Natl. Cancer Inst. 1999, 91, 1688-1689.

79. Breen, M. Canine Cytogenetics - From Band to Basepair. Cytogenet. Genome Res. 2008, 120, $50-60$.

80. Breen, M.; Modiano, J.F. Evolutionarily Conserved Cytogenetic Changes in Hematological Malignancies of Dogs and Humans-Man and His Best Friend Share More than Companionship. Chromosome Res. 2008, 16, 145-154. 
81. Hedan, B.; Thomas, R.; Motsinger-Reif, A.; Abadie, J.; Andre, C.; Cullen, J.; Breen, M. Molecular Cytogenetic Characterization of Canine Histiocytic Sarcoma: A Spontaneous Model for Human Histiocytic Cancer Identifies Deletion of Tumor Suppressor Genes and Highlights Influence of Genetic Background on Tumor Behavior. BMC Cancer 2011, 11, 201.

82. Breen, M.; Thomas, A. Canine Cytogenetics and Chromosome Maps. In Genetics of the Dog, 2nd ed.; Ostrander, E.A., Ruvinsky, A., Eds.; CAB International: London, UK, 2012; pp. $245-254$.

83. Daniel, J.; Coulter, J.; Woo, J.H.; Wilsbach, K.; Gabrielson, E. High Levels of the Mps1 Checkpoint Protein are Protective of Aneuploidy in Breast Cancer Cells. Proc. Natl. Acad. Sci. USA 2011, 108, 5384-5389.

84. Solomon, D.A.; Kim, T.; Diaz-Martinez, L.A.; Fair, J.; Elkahloun, A.G.; Harris, B.T.; Toretsky, J.A.; Rosenberg, S.A.; Shukla, N.; Ladanyi, M.; et al. Mutational Inactivation of STAG2 Causes Aneuploidy in Human Cancer. Science 2011, 333, 1039-1043.

85. Kramer, A.; Maier, B.; Bartek, J. Centrosome Clustering and Chromosomal (in)Stability: A Matter of Life and Death. Mol. Oncol. 2011, 5, 324-335.

86. Bunz, F.; Fauth, C.; Speicher, M.R.; Dutriaux, A.; Sedivy, J.M.; Kinzler, K.W.; Vogelstein, B.; Lengauer, C. Targeted Inactivation of p53 in Human Cells does Not Result in Aneuploidy. Cancer Res. 2002, 62, 1129-1133.

87. Li, M.; Fang, X.; Baker, D.J.; Guo, L.; Gao, X.; Wei, Z.; Han, S.; van Deursen, J.M.; Zhang, P. The ATM-p53 Pathway Suppresses Aneuploidy-Induced Tumorigenesis. Proc. Natl. Acad. Sci. USA 2010, 107, 14188-14193.

88. Aylon, Y.; Oren, M. P53: Guardian of Ploidy. Mol. Oncol. 2011, 5, 315-323.

89. Nasir, L.; Rutteman, G.R.; Reid, S.W.; Schulze, C.; Argyle, D.J. Analysis of p53 Mutational Events and MDM2 Amplification in Canine Soft-Tissue Sarcomas. Cancer Lett. 2001, 174, 83-89.

90. Kirpensteijn, J.; Kik, M.; Teske, E.; Rutteman, G.R. TP53 Gene Mutations in Canine Osteosarcoma. Vet. Surg. 2008, 37, 454-460.

91. Fabarius, A.; Li, R.; Yerganian, G.; Hehlmann, R.; Duesberg, P. Specific Clones of Spontaneously Evolving Karyotypes Generate Individuality of Cancers. Cancer Genet. Cytogenet 2008, 180, 89-99.

92. Affolter, V.K.; Moore, P.F. Localized and Disseminated Histiocytic Sarcoma of Dendritic Cell Origin in Dogs. Vet. Pathol. 2002, 39, 74-83.

93. Maas, C.P.; ter Haar, G.; van der Gaag, I.; Kirpensteijn, J. Reclassification of Small Intestinal and Cecal Smooth Muscle Tumors in 72 Dogs: Clinical, Histologic, and Immunohistochemical Evaluation. Vet. Surg. 2007, 36, 302-313.

94. Vindelov, L.L.; Christensen, I.J.; Nissen, N.I. A Detergent-Trypsin Method for the Preparation of Nuclei for Flow Cytometric DNA Analysis. Cytometry 1983, 3, 323-327.

95. Canis Lupus familiaris tumor protein p53 Available online: http://www.ncbi.nlm.nih.gov/ nuccore/NM_001003210.1/ (accessed on 16 November 2012). 

MDPI AG

Klybeckstrasse 64

4057 Basel, Switzerland

Tel. +41 616837734

Fax +41613028918

http://www.mdpi.com/

Cancers Editorial Office

E-Mail: cancers@mdpi.com

http://www.mdpi.com/journal/cancers 

回裂回 MDPI - Basel • Beijing - Wuhan 程 ISBN 978-3-906980-58-4

茴经 www.mopi.com 